\title{
Aplicações da teoria dos espaços coarse a espaços de Banach e grupos topológicos
}

\author{
Denis de Assis Pinto Garcia
}

\author{
DISSERTAÇÃO APRESENTADA \\ $\mathrm{AO}$ \\ Instituto DE MATEMÁticA E EstatísticA \\ DA \\ UniversidAde DE SÃo PAUlo \\ PARA \\ OBTENÇÃO DO TÍTULO \\ $\mathrm{DE}$ \\ MESTRE EM CiÊNCIAS
}

\author{
Programa: Matemática \\ Orientador: Prof. Dr. Valentin Raphael Henri Ferenczi
}

Durante o desenvolvimento deste trabalho, o autor recebeu auxílio financeiro do CNPq. 


\section{Aplicações da teoria dos espaços coarse a espaços de Banach e grupos topológicos}

Esta versão da dissertação contém as correções e alterações sugeridas pela Comissão Julgadora durante a defesa da versão original do trabalho, realizada em 24/06/2019. Uma cópia da versão original está disponível no

Instituto de Matemática e Estatística da Universidade de São Paulo.

Comissão Julgadora:

- Prof. Dr. Valentin Raphael Henri Ferenczi - IME-USP

- Prof. Dr. Willian Hans Goes Corrêa - IME-USP

- Prof. Dr. Leandro Candido Batista - ICT-UNIFESP 


\section{Agradecimentos}

Agradeço, inicialmente, a Deus, por permitir que eu chegasse até aqui.

Em segundo lugar, agradeço à minha mãe, Rosely, por sempre me dar todo o incentivo e apoio necessários para empreender um projeto como este; ao meu pai, Celso, por todas as conversas e conselhos, e por ter sempre acreditado em mim; às minhas avós, Ruth e Mafalda, por todo o amor, carinho e incentivo ao longo dos anos; e ao meu avô, David, pelas nossas conversas, e por ter sempre me motivado a seguir em frente.

O que seria de um homem sem amigos? Agradeço, portanto, aos meus amigos, com os quais sempre pude contar nos momentos de tristeza e de alegria, por todas as inestimáveis horas de conversas, risadas e conselhos, e por terem sempre acreditado no meu potencial e me motivado a perseguir os meus objetivos. Faço, ainda, um agradecimento especial ao meu grande amigo, Matheus Lazarotto, pelo auxílio na elaboração das figuras, e aos meus queridos amigos, Arthur Müller, Laïs de Campos e Juliane Trianon Fraga, pela ajuda com a revisão de texto.

Agradeço, também, ao professor Valentin, por ter-me dado a oportunidade de ser seu aluno, pelos valiosos conselhos e sugestões, e pela dedicação com que acompanhou a produção deste trabalho; e a todos aqueles que, de alguma forma, contribuíram para a minha educação: aos meus pais, que sempre se esforçaram para me proporcionar um ensino de qualidade; aos meus professores, os quais sempre instigaram em mim a curiosidade e o desejo de aprender; e, é claro, aos meus amigos, sem os quais as infindáveis horas de estudo com certeza teriam sido muito mais penosas.

E, por fim, agradeço ao CNPq e ao Instituto de Matemática e Estatística da Universidade de São Paulo pelo auxílio financeiro. 


\section{Resumo}

GARCIA, D. A. P. Aplicações da teoria dos espaços coarse a espaços de Banach e grupos topológicos. 2019. 134 p. Dissertação (Mestrado) - Instituto de Matemática e Estatística, Universidade de São Paulo, São Paulo, 2019.

Este trabalho é uma contribuição ao estudo da geometria de larga escala de espaços de Banach e de grupos topológicos. Embora esses dois campos sejam tradicionalmente estudados de forma independente, em 2017, Christian Rosendal mostrou que eles podem ser encarados como faces distintas de algo maior: a geometria grosseira de grupos topológicos. Uma ferramenta essencial para o desenvolvimento dessa nova abordagem é a noção de estrutura coarse, introduzida por John Roe em 2003, a qual pode ser vista como a contraparte de larga escala do conceito de estrutura uniforme. Por essa razão, os capítulos iniciais da dissertação destinam-se a apresentar uma introdução elementar à teoria dos espaços uniformes e dos espaços coarse, destacando os conceitos-chave para a compreensão dos demais capítulos e conferindo particular atenção ao estudo de uniformidades e estruturas coarse associadas a grupos topológicos, dentre as quais são enfatizadas as estruturas uniforme à esquerda e coarse à esquerda de um grupo topológico. No capítulo 5, são discutidos resultados recentes de Christian Rosendal acerca da existência de mergulhos uniformes e mergulhos grosseiros entre espaços de Banach. Dois dos mais importantes afirmam que, se existir uma função $\sigma$ uniformemente contínua e não colapsada entre os espaços de Banach $\left(X,\|\cdot\|_{X}\right)$ e $\left(E,\|\cdot\|_{E}\right)$, então, para todo $p \in[1,+\infty[$, existirá um mergulho uniforme de $\left(X,\|\cdot\|_{X}\right)$ em $\left(\ell_{p}(E),\|\cdot\|_{p}\right)$ o qual é, também, um mergulho grosseiro, e que, se $\sigma$ for, também, limitada, existirá um mergulho grosseiro uniformemente contínuo de $\left(X,\|\cdot\|_{X}\right)$ em $\left(E \times E,\|\cdot\|_{E \times E}\right)$. Já no capítulo 6, estuda-se a classe das estruturas coarse invariantes à esquerda sobre grupos. Inicialmente, mostra-se como uma estrutura coarse invariante à esquerda em um grupo $(G, \cdot)$ pode ser descrita em função de um certo ideal sobre $G$, e viceversa. Em seguida, utiliza-se esse resultado para caracterizar a estrutura coarse à esquerda $\mathcal{E}_{L}$ de um grupo topológico $(G, \cdot, \tau)$ em termos da coleção dos conjuntos grosseiramente limitados em $\left(G, \mathcal{E}_{L}\right)$ e, com isso, provar que a estrutura coarse à esquerda associada ao grupo aditivo de um espaço normado coincide com a estrutura coarse limitada induzida pela norma.

Palavras-chave: espaços uniformes, espaços coarse, mergulhos uniformes, mergulhos grosseiros, geometria de larga escala, espaços de Banach, grupos topológicos. 


\section{Abstract}

GARCIA, D. A. P. [Applications of coarse spaces theory to Banach spaces and topological groups]. 2019. 134 p. Dissertation (Master's degree) — Instituto de Matemática e Estatística, Universidade de São Paulo, São Paulo, 2019. Portuguese.

This work is a contribution to the study of large-scale geometry of Banach spaces and topological groups. Although these two fields are traditionally studied independently, in 2017, Christian Rosendal showed they can be regarded as different aspects of a more general theory: the coarse geometry of topological groups. An essential tool for the development of this new approach is the notion of coarse structure, introduced by John Roe in 2003, which can be seen as the large-scale counterpart of the concept of uniform structure. For this reason, the initial chapters of this work intend to present an elementary introduction to both uniform and coarse spaces theory, highlighting the key concepts for the understanding of the other chapters and paying particular attention to the study of uniform and coarse structures associated with topological groups, and, mainly, to the left-uniform and the left-coarse structures of a topological group. In Chapter 5, we discuss Rosendal's recent results on the existence of uniform and coarse embeddings between Banach spaces. Two of the most important state that, if there is an uncollapsed uniformly continuous function $\sigma$ between the Banach spaces $\left(X,\|\cdot\|_{X}\right)$ and $\left(E,\|\cdot\|_{E}\right)$, then, for all $p \in\left[1,+\infty\left[,\left(X,\|\cdot\|_{X}\right)\right.\right.$ admits a simultaneously uniform and coarse embedding into $\left(\ell_{p}(E),\|\cdot\|_{p}\right)$, and that, if, in addition, we assume that $\sigma$ maps into a bounded set, then $\left(X,\|\cdot\|_{X}\right)$ also admits a uniformly continuous coarse embedding into $\left(E \times E,\|\cdot\|_{E \times E}\right)$. On the other hand, in chapter 6 , we focus our attention on the class of left-invariant coarse structures on groups. In the first section, we show how a left-invariant coarse structure on a group $(G, \cdot)$ can be described in terms of a certain ideal on $G$, and vice versa. After that, we use this result to characterize the left-coarse structure $\mathcal{E}_{L}$ of a topological group $(G, \cdot, \tau)$ in terms of the collection of the coarsely bounded sets of $\left(G, \mathcal{E}_{L}\right)$ and, with this, we prove that the left-coarse structure associated with the additive group of a normed space is simply the bounded coarse structure induced by its norm.

Keywords: uniform spaces, coarse spaces, uniform embeddings, coarse embeddings, large-scale geometry, Banach spaces, topological groups. 


\section{Sumário}

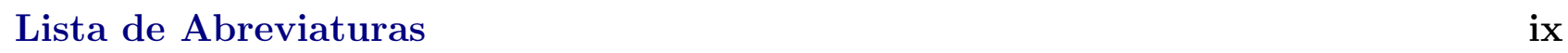

Lista de Símbolos $\quad$ xi

Lista de Figuras $\quad$ XV

1 INTRODUÇÃO 1

2 Preliminares $\quad 5$

2.1 NoTAÇÃO . . . . . . . . . . . . . . . . . . . . . . . . . 5

2.2 RelaÇÕes binárias Sobre um CONJUnto . . . . . . . . . . . . . . 5

2.3 EstaÇos métricos e pSEUdométricos . . . . . . . . . . . . . . 7

2.4 SequênCIAS E SÉries de nÚMEROS REAis . . . . . . . . . . . . . . . 11

2.5 ESPAÇOS VETORIAIS E TRANSFORMAÇÕES LINEARES . . . . . . . . . . . . . 12

2.6 ESPAÇOS NORMADOS . . . . . . . . . . . . . . . . . 15

2.7 TopoloGiA . . . . . . . . . . . . . . . . . . . . . 18

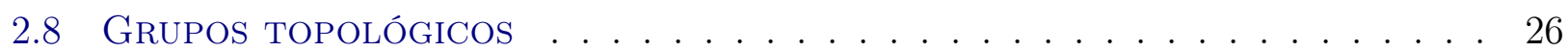

3 EspaÇos Uniformes $\quad 29$

3.1 Estruturas Uniformes . . . . . . . . . . . . . . . . . . 30

3.2 A ESTRUtura Uniforme À ESQUERdA de UM GRUPO TOPOLÓGiCO . . . . . 37

3.3 TOPOLOGIAS INDUZIDAS POR ESTRUTURAS UNIFORMES . . . . . . . . . . . 41

3.4 FunÇÕes entre EstaÇOS Uniformes . . . . . . . . . . . . . . . . . 46

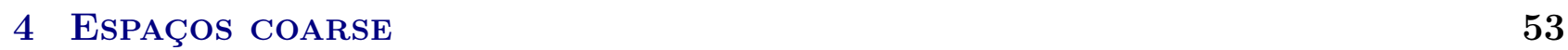

4.1 Estruturas COARSE . . . . . . . . . . . . . . . . . 54

4.2 A estrutura cotrse gerada por uma coleção . . . . . . . . . . . . . 61

4.3 A estrutura coarse à esquerda de um Grupo topológico . . . . . . . 63

4.4 FunÇÕes Entre eSPAÇOS COARSE . . . . . . . . . . . . . . . . . . . 64

5 Mergulhos entre espaÇos de BanACH $\quad 81$

$5.1 \quad \mathrm{O}$ ESPAÇO $E \times F \ldots \ldots \ldots \ldots \ldots$

$5.2 \mathrm{O}$ ESPAÇO $\ell_{p}(E) \ldots \ldots \ldots \ldots \ldots$ 
viii SUMÁRIO

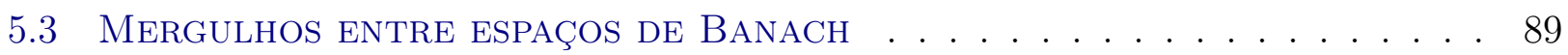

6 Estruturas COARSE INVARIANTES À ESQUERDA 105

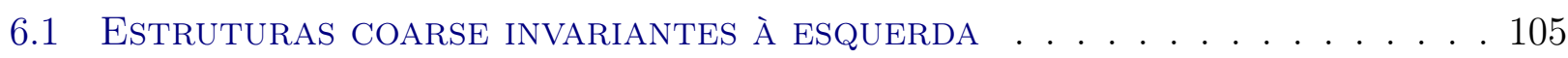

6.2 Conjuntos grosseiramente limitados . . . . . . . . . . . . . . . . 112

6.3 A estrutura CoArse À esquerda do Grupo Aditivo de um espaÇo

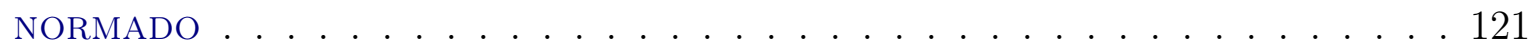

A Uniformidades INVARIANTEs À ESQUERda $\quad \mathbf{1 2 5}$

A.1 UniformidAdes inVARIANTES À ESQUERDA . . . . . . . . . . . . . . . . . 125

A.2 Relacionando Uniformidades a filtros . . . . . . . . . . . . . 126

$\begin{array}{ll}\text { Referências Bibliográficas } & 131\end{array}$

$\begin{array}{ll}\text { Índice Remissivo } & 132\end{array}$ 


\section{Lista de Abreviaturas}

E.N. espaço normado;

resp. respectivamente;

t.q. tal que ou tais que. 


\section{Lista de Símbolos}

$X, Y \quad$ Em geral, denotam conjuntos não vazios;

$\Delta(X) \quad\{(x, x): x \in X\} \quad[$ Diagonal de $X]$;

$E, F \quad$ Em geral, denotam subconjuntos de $X \times X$;

$E^{-1} \quad\{(x, y) \in X \times X:(y, x) \in E\} ;$

$E \circ F \quad\{(x, y) \in X \times X: \exists z \in X$ t.q. $(x, z) \in F$ e $(z, y) \in E\}$;

$\mathscr{D}_{d} \quad$ Uniformidade induzida por uma pseudométrica $d$;

$D_{\varepsilon}^{d}, E_{\varepsilon}^{d} \quad\{(x, y) \in X \times X: d(x, y)<\varepsilon\}$, em que $d$ é uma pseudométrica sobre $X$;

$\mathcal{E}_{d} \quad$ Estrutura coarse limitada associada a uma pseudométrica $d$;

$\mathbb{R} \quad$ Conjunto (ou corpo) dos números reais;

$\mathbb{Z} \quad$ Conjunto dos números inteiros;

$\mathbb{K} \quad$ Corpo dos números reais ou dos números complexos;

$\ell_{p}\left(E,\|\cdot\|_{E}\right) \quad\left\{\left(x_{n}\right)_{n \in \mathbb{N}}: x_{n} \in E \forall n \in \mathbb{N}\right.$ e $\left.\left(\left\|x_{n}\right\|_{E}\right)_{n \in \mathbb{N}} \in \ell_{p}\right\}$, em que $\left(E,\|\cdot\|_{E}\right)$ é um espaço normado sobre $\mathbb{K}$ e $p \in[1,+\infty]$;

$\ell_{p} \quad$ Espaço vetorial das sequências $\left(x_{n}\right)_{n \in \mathbb{N}} \in \mathbb{K}^{\mathbb{N}}$ tais que $\sum_{n=1}^{+\infty}\left|x_{n}\right|^{p}$ converge, em que $p \in[1,+\infty[$, munido das operações usuais de soma e de multiplicação por escalar de sequências;

$\mathbb{C} \quad$ Conjunto (ou corpo) dos números complexos;

$\mathbb{Q} \quad$ Conjunto (ou corpo) dos números racionais;

$\mathbb{N} \quad\{1,2,3, \ldots\}$

$\mathbb{N}_{0} \quad \mathbb{N} \cup\{0\} ;$

$\operatorname{Gr}(f) \quad$ Gráfico da função $f$;

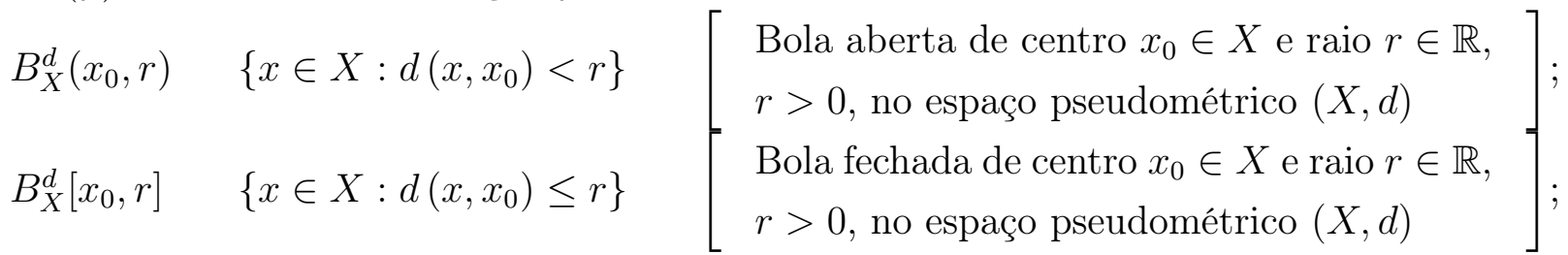

$\operatorname{diam}_{d}(A) \quad$ Diâmetro de um subconjunto não vazio $A \subseteq X$ em relação a uma pseudométrica $d$ sobre $X$;

$0_{V} \quad$ Vetor nulo de um espaço vetorial $V$ sobre um corpo $\mathbb{K}$;

$\mathbb{K}^{\mathbb{N}} \quad$ Coleção das sequências de elementos de $\mathbb{K}$;

$\ell_{\infty} \quad$ Espaço vetorial das sequências limitadas em $\mathbb{K}$, munido das operações usuais de soma e de multiplicação por escalar; 
$c_{0} \quad$ Espaço vetorial das sequências de elementos de $\mathbb{K}$ que convergem a 0, munido das operações usuais;

$W_{\mathcal{S}} \quad$ Subespaço vetorial gerado por $\mathcal{S}$

$\operatorname{Ker}(T) \quad$ Núcleo da transformação linear $T$;

$\operatorname{Im}(f) \quad$ Imagem da função $f$;

$\delta_{i j} \quad$ Delta de Kronecker;

$d_{\|\cdot\|_{X}} \quad$ Métrica induzida por $\|\cdot\|_{X}$ sobre $X$;

$\|T\| \quad$ Norma de uma transformação linear $T$;

$\mathcal{P}(X) \quad$ Conjunto das partes de $X$;

$\tau_{A} \quad\{U \cap A: U \in \tau\}$, em que $(X, \tau)$ é um espaço topológico e $A \subseteq X$ é um subconjunto não vazio;

$\tau_{d} \quad$ Topologia induzida por uma pseudométrica $d$;

$\tau_{\|\cdot\|} \quad$ Topologia induzida por uma norma $\|\cdot\|$ (isto é, $\tau_{\|\cdot\|}:=\tau_{d_{\|\cdot\|}}$ );

$\operatorname{int}_{\tau}(A) \quad$ Interior de um conjunto $A \subseteq X$ em um espaço topológico $(X, \tau)$;

$\bar{A}^{\tau} \quad$ Fecho de um conjunto $A \subseteq X$ em um espaço topológico $(X, \tau)$;

e Elemento neutro de um $\operatorname{grupo}(G, \cdot)$;

$D_{a} \quad \Delta(X) \cup\left\{(x, y) \in \mathbb{R}^{2}: x>a\right.$ e $\left.y>a\right\}$, em que $a \in \mathbb{R} ;$

$\mathscr{D}_{A} \quad\left\{D \cap(A \times A): D \in \mathscr{D}_{X}\right\}$, em que $\left(X, \mathscr{D}_{X}\right)$ é um espaço uniforme e $A \subseteq X$ é um subconjunto não vazio;

$\mathcal{V} \quad$ Coleção das vizinhanças de $e$ em $(G, \tau)$, em que $(G, \cdot, \tau)$ é um grupo topológico; $E_{A} \quad\left\{(x, y) \in G \times G: x^{-1} y \in A\right\}$, em que $(G, \cdot)$ é um grupo e $A \subseteq G ;$

$\mathscr{L} \quad\left\{E_{V}: V \in \mathcal{V}\right\}$;

$\mathcal{U}_{L} \quad$ Estrutura uniforme à esquerda associada a um grupo topológico;

$\mathcal{U}_{R} \quad$ Estrutura uniforme à direita associada a um grupo topológico;

$\mathscr{R} \quad\left\{R_{V}: V \in \mathcal{V}\right\}$;

$R_{V} \quad\left\{(x, y) \in G \times G: y x^{-1} \in V\right\}$, em que $(G, \cdot)$ é um grupo e $V \subseteq G$;

$D[K] \quad\{y \in X: \exists x \in K$ t.q. $(x, y) \in D\}$, em que $K \subseteq X$ e $D \subseteq X \times X$;

$\tau_{\mathscr{D}} \quad$ Topologia induzida por uma uniformidade $\mathscr{D}$;

$F_{f} \quad\{(x, y) \in X \times X:(f(x), f(y)) \in F\}$, em que $f: X \longrightarrow Y$ é uma função e $F \subseteq Y \times Y$

$E_{f} \quad\{(f(x), f(y)):(x, y) \in E\}$, em que $E \subseteq X \times X$ e $f: X \longrightarrow Y$ é uma função;

id Função identidade;

$\mathcal{E}_{\max } \quad \mathcal{P}(X \times X) \quad$ (Estrutura coarse maximal sobre $\left.X\right)$;

$\mathcal{E}_{\text {min }} \quad \mathcal{P}(\Delta(X)) \quad($ Estrutura coarse minimal sobre $X) ;$

$\mathcal{E}_{A} \quad\left\{E \subseteq A \times A: E \in \mathcal{E}_{X}\right\}$, em que $\left(X, \mathcal{E}_{X}\right)$ é um espaço coarse e $A \subseteq X$ é um subconjunto não vazio;

$\mathcal{E}_{i} \quad$ Estrutura coarse indiscreta sobre $X$;

$\mathcal{E}_{\mathcal{S}} \quad$ Estrutura coarse gerada por uma coleção $\mathcal{S} ;$

$\mathcal{D} \quad$ Coleção das pseudométricas contínuas e invariantes à esquerda de um grupo topológico; 
$\mathcal{E}_{L} \quad$ Estrutura coarse à esquerda associada a um grupo topológico;

$L(A, M) \quad\left\{f: A \longrightarrow M: \operatorname{diam}_{d}(\operatorname{Im}(f))<+\infty\right\}$, em que $(M, d)$ é um espaço métrico e $A$ é um conjunto não vazio;

$\underset{\text { prox }}{\sim} \quad$ Relação de proximidade;

$\mathscr{F}(S, X) \quad$ Coleção das funções de $S$ em $X$, em que $S$ é um conjunto não vazio;

$d_{\|\cdot\|_{\mathbb{Z}}}, d_{\|\cdot\|_{\mathbb{Q}}}$ Restrição de $d_{\|\cdot\| \cdot \|}$, respectivamente, a $\mathbb{Z} \times \mathbb{Z}$ e a $\mathbb{Q} \times \mathbb{Q}$;

$A_{E} \quad\{x \in G:(e, x) \in E\}$, em que $(G, \cdot)$ é um grupo e $E \subseteq G \times G$ é invariante à esquerda em $(G, \cdot)$;

$\mathcal{A}_{\mathcal{E}} \quad\left\{A \subseteq G: E_{A} \in \mathcal{E}\right\}$, em que $(G, \cdot)$ é um grupo e $\mathcal{E}$ é uma estrutura coarse sobre $G$;

$\mathcal{E}_{\mathcal{A}} \quad\left\{E \subseteq G \times G: \exists A \in \mathcal{A}\right.$ t.q. $\left.E \subseteq E_{A}\right\}$, em que $(G, \cdot)$ é um grupo e $\mathcal{A} \subseteq \mathcal{P}(G) ;$

$\mathcal{C B}_{\mathcal{E}}$ $\{A \subseteq X: A \times A \in \mathcal{E}\} \quad\left[\begin{array}{l}\text { Coleção dos conjuntos grosseiramente } \\ \text { limitados em }(X, \mathcal{E})\end{array}\right]$; $\mathcal{O B} \quad \mathcal{C B}_{\mathcal{E}_{L}}$

$n \star A \quad \underbrace{A+\ldots+A}_{n \text { vezes }}$, em que $n \in \mathbb{N}$ e $A \in V$, sendo $V$ um espaço vetorial sobre $\mathbb{K}$;

$\mathscr{F}_{\mathscr{D}} \quad\left\{F \subseteq G: E_{F} \in \mathscr{D}\right\}$, em que $(G, \cdot)$ é um grupo e $\mathscr{D}$ é uma estrutura uniforme sobre $G$;

$\mathscr{D}_{\mathscr{F}} \quad\left\{D \subseteq G \times G: \exists F \in \mathscr{F}\right.$ t.q. $\left.E_{F} \subseteq D\right\}$, em que $(G, \cdot)$ é um grupo e $\mathscr{F} \subseteq \mathcal{P}(G)$. 


\section{Lista de Figuras}

$1.1 \mathbb{R}$ e $\mathbb{Z}$ são os mesmos do ponto de vista da geometria de larga escala. . . . . . . 3

3.1 Base da uniformidade usual sobre $\mathbb{R} . \quad \ldots \ldots \ldots$. . . . . . . . . . . . . 32

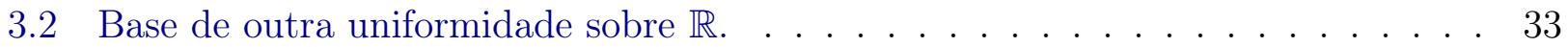

3.3 Vizinhança diagonal da uniformidade usual sobre $\mathbb{R} \ldots \ldots$. . . . . . . . . . . 36

3.4 Representação de $D[x] \ldots \ldots \ldots$. . . . . . . . . . . . . . . . . 43

4.1 Conjunto controlado da estrutura coarse usual sobre $\mathbb{R}$. . . . . . . . . . . . . 59

4.2 Um outro exemplo de conjunto controlado. . . . . . . . . . . . . . . . 59

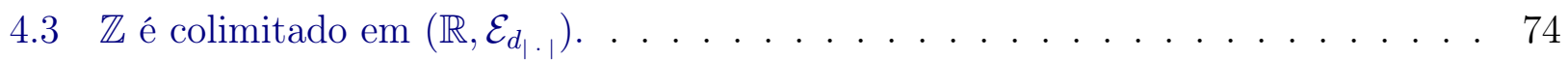

$4.4 \mathbb{Z}^{2}$ é colimitado em $\left(\mathbb{R}^{2}, \mathcal{E}_{d_{\|\cdot\|}}\right) \ldots \ldots \ldots \ldots \ldots \ldots \ldots$

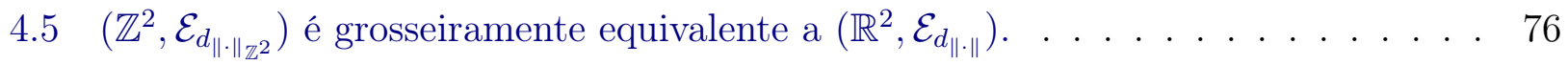


xvi LISTA DE FIGURAS 


\section{Capítulo 1}

\section{INTRODUÇÃO}

Este trabalho é uma contribuição ao estudo da geometria não linear de espaços de Banach e da geometria de larga escala de grupos topológicos. Embora esses dois campos possuam muitos aspectos em comum, são, tradicionalmente, estudados de forma independente. Contudo, em um trabalho recente [Ros17a], Christian Rosendal mostrou que ambas as teorias podem ser encaradas como parte de algo maior: a geometria grosseira de grupos topológicos. Assim sendo, muitos problemas os quais admitem tratamentos análogos, mas separados, para espaços de Banach e grupos topológicos podem ser analisados por meio dessa abordagem unificadora.

Uma ferramenta essencial para o desenvolvimento dessa nova abordagem é a noção de estrutura coarse, ${ }^{1}$ introduzida por John Roe em 2003 [Roe03], a qual pode ser vista como a contraparte de larga escala do conceito de estrutura uniforme. Mais especificamente, uma estrutura coarse sobre um conjunto não vazio $X$ é uma coleção $\mathcal{E}$ de subconjuntos de $X \times X$ com as seguintes propriedades:

a) $\Delta(X):=\{(x, x): x \in X\} \in \mathcal{E}$

b) Se $E, F \in \mathcal{E}$, então $E \cup F \in \mathcal{E}$;

c) Se $E \in \mathcal{E}$, então $E^{-1}:=\{(x, y) \in X \times X:(y, x) \in E\} \in \mathcal{E}$;

d) Se $E, F \in \mathcal{E}$, então $E \circ F:=\{(x, y) \in X \times X: \exists z \in X$ t.q. $(x, z) \in F$ e $(z, y) \in E\} \in \mathcal{E}$;

e) Se $E \in \mathcal{E}$ e $F \subseteq E$, então $F \in \mathcal{E}$.

E, por sua vez, um espaço coarse nada mais é do que um par $(X, \mathcal{E})$, em que $X$ é um conjunto não vazio e $\mathcal{E}$ é uma estrutura coarse sobre $X$.

O exemplo motivador (e, com certeza, um dos mais importantes) para o estudo dos espaços uniformes e dos espaços coarse é, sem dúvidas, aquele em que $(X, d)$ é um espaço métrico. Com efeito, dada uma métrica $d$ sobre um conjunto não vazio $X$, é fácil ver que a coleção

$$
\mathscr{D}_{d}:=\left\{D \subseteq X \times X: \exists \alpha>0 \text { t.q. } E_{\alpha}^{d} \subseteq D\right\},
$$

\footnotetext{
${ }^{1}$ O termo "coarse", que, em tradução literal, significa "grosseiro", não possui, até o momento, um equivalente canônico em português. Por essa razão, foi adotado o seguinte procedimento ao longo da escrita deste documento: nos casos em que a tradução de "coarse" como "grosseiro" é boa (como em "mergulho grosseiro" e "equivalência grosseira"), ela foi utilizada. Naqueles casos, porém, em que o termo "grosseiro" não reflete bem a intuição por trás dos conceitos em questão (como em "estrutura coarse" e "espaço coarse"), optou-se por manter o termo "coarse" no original. Uma boa alternativa, nesses casos, seria utilizar as expressões "estrutura de larga escala" e "espaço de larga escala" em substituição, respectivamente, a "estrutura grosseira" e a "espaço grosseiro", dado que tais expressões capturam o verdadeiro sentido por trás dos termos citados. Apesar disso, por se tratar de termos novos, sem ocorrências prévias na literatura, decidiu-se por não utilizá-los neste primeiro trabalho sobre o tema, uma vez que, por serem desconhecidos, seu uso poderia contribuir para a diminuição da visibilidade da dissertação (em buscas online, por exemplo).
} 
em que, para cada $\alpha>0$,

$$
E_{\alpha}^{d}:=\{(x, y) \in X \times X: d(x, y)<\alpha\}
$$

é uma uniformidade sobre $X$. Da mesma forma, não é difícil mostrar que

$$
\mathcal{E}_{d}:=\left\{E \subseteq X \times X: \exists \alpha>0 \text { t.q. } E \subseteq E_{\alpha}^{d}\right\}
$$

é uma estrutura coarse sobre $X$. Dizemos que $\mathscr{D}_{d}$ e $\mathcal{E}_{d}$ são, respectivamente, a uniformidade e a estrutura coarse limitada induzidas por $d$ sobre $X$.

Outros exemplos particularmente importantes para o desenvolvimento do presente trabalho são as uniformidades e estruturas coarse associadas a grupos topológicos. Como exemplos mais concretos, podemos citar, respectivamente, as estruturas uniforme à esquerda e coarse à esquerda de um grupo topológico, ambas as quais são estudadas em detalhes na dissertação.

É fundamental ressaltar que o estudo dos espaços coarse é, ainda, bastante recente, e está em pleno desenvolvimento, de modo que há, até o momento, relativamente poucos trabalhos sobre o assunto. Por essa razão, reservamos os primeiros capítulos da dissertação para introduzir, de forma elementar, um pouco da terminologia e alguns dos principais conceitos subjacentes a essa teoria. Para que essa apresentação se dê da forma mais natural possível, recorremos à seguinte estratégia: inicialmente, apresentamos uma introdução aos espaços uniformes, os quais, por sua vez, já estão substancialmente mais consolidados, e, em seguida, exploramos uma certa dualidade entre as definições de estrutura uniforme e de estrutura coarse para motivar as ideias e introduzir os conceitos relativos à teoria dos espaços coarse tendo como inspiração as suas respectivas contrapartes na teoria dos espaços uniformes. Nos próximos parágrafos, vamos comentar, de forma um pouco mais detalhada, acerca da estrutura dos capítulos iniciais da dissertação.

No segundo capítulo, fazemos algumas definições e considerações preliminares e enunciamos alguns resultados básicos de Topologia e de Análise Funcional, os quais são importantes para a compreensão do restante do tabalho.

No capítulo seguinte, introduzimos o conceito de estrutura uniforme sobre um conjunto arbitrário e discutimos alguns exemplos, dentre os quais se destacam a uniformidade induzida por uma pseudométrica e a estrutura uniforme à esquerda associada a um grupo topológico. Posteriormente, mostramos como uma uniformidade sobre um conjunto não vazio $X$ induz uma topologia sobre $X$, e verificamos, entre outras coisas, que a topologia induzida pela estrutura uniforme à esquerda de um grupo topológico coincide com a topologia do grupo. Por fim, encerramos o capítulo com uma seção dedicada ao estudo de algumas classes especiais de funções entre espaços uniformes. Nessa última seção, introduzimos e caracterizamos a continuidade uniforme e também os conceitos de mergulho e homeomorfismo uniformes.

O quarto capítulo encerra a parte inicial da dissertação. Iniciamos o capítulo definindo o conceito de estrutura coarse sobre um conjunto $X$ e apresentando alguns exemplos simples, dentre os quais o mais importante é, sem dúvidas, a estrutura coarse limitada associada a uma pseudométrica. A seguir, definimos e caracterizamos a estrutura coarse gerada por uma coleção $\mathcal{S}$ de subconjuntos de $X \times X$, e introduzimos a estrutura coarse à esquerda de um grupo topológico $(G, \cdot, \tau)$. A última seção do capítulo dedica-se ao estudo de alguns tipos especiais de funções entre espaços coarse e das relações entre eles. Nessa seção, os conceitos de bornologia, expansividade, mergulho grosseiro e equivalência grosseira, originalmente aplicáveis a funções entre espaços pseudométricos, são redefinidos no contexto mais geral das funções entre espaços coarse, conferindo um significado preciso a afirmações como, por exemplo, a de que " $\mathbb{R}$ e $\mathbb{Z}$ são os mesmos sob uma perspectiva de larga escala", as quais são, a priori, concebidas a partir da intuição. 


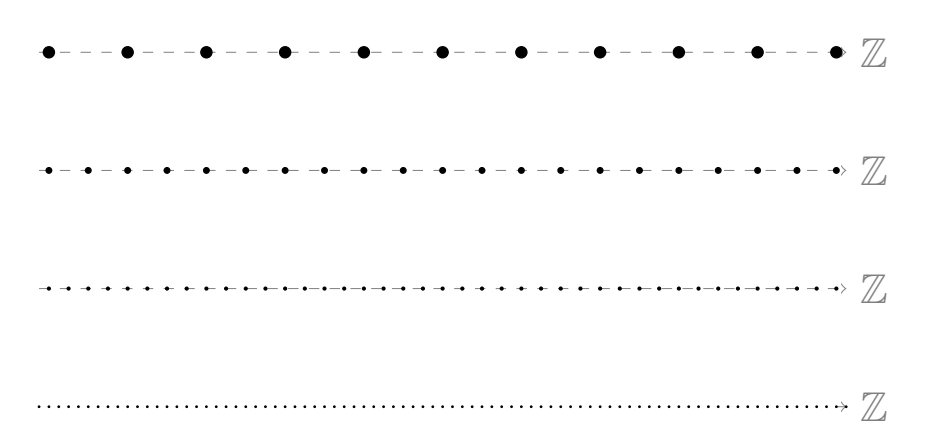

Figura 1.1: A figura acima ilustra a ideia intuitiva de que $\mathbb{Z}$ se parece com $\mathbb{R}$ quando observado a partir de grandes distâncias.

Após termos estabelecido uma base teórica conveniente, embora ainda bastante elementar, nos capítulos 2, 3 e 4, passamos a nos concentrar em duas situações um pouco mais concretas. No capítulo 5, estudamos questões relativas à existência de mergulhos uniformes e mergulhos grosseiros entre espaços de Banach. E, no capítulo 6, focamos nossa atenção no estudo de estruturas coarse invariantes à esquerda associadas a grupos, e, em particular, na caracterização da estrutura coarse à esquerda $\mathcal{E}_{L}$ de um grupo topológico $(G, \cdot, \tau)$ em termos da coleção dos conjuntos grosseiramente limitados em $\left(G, \mathcal{E}_{L}\right)$. Nos próximos parágrafos, comentaremos, de forma breve, a respeito de alguns dos principais tópicos discutidos em cada uma dessas direções.

Em um artigo de 2008 [Kal08], N. J. Kalton observa que dois espaços de Banach, $\left(X,\|\cdot\|_{X}\right)$ e $\left(E,\|\cdot\|_{E}\right)$, de dimensão infinita sobre um mesmo corpo $\mathbb{K},{ }^{2}$ e uniformemente homeomorfos, são, também, grosseiramente equivalentes. Tendo isso em vista, é natural perguntar se um resultado como esse vale também para mergulhos. Isto é:

Questão 1: Se $\left(X,\|\cdot\|_{X}\right)$ e $\left(E,\|\cdot\|_{E}\right)$ são espaços de Banach tais que $\left(X,\|\cdot\|_{X}\right)$ pode ser uniformemente mergulhado em $\left(E,\|\cdot\|_{E}\right)$, podemos garantir que existe também um mergulho grosseiro de $\left(X,\|\cdot\|_{X}\right)$ em $\left(E,\|\cdot\|_{E}\right)$ ?

De modo geral, essa pergunta permanece em aberto. Apesar disso, em 2017, Christian Rosendal demonstrou uma série de resultados que ajudam a respondê-la em certos casos particulares [Ros17b], alguns dos quais discutimos no capítulo 5 da dissertação. Um dos mais importantes afirma que, se $\left(X,\|\cdot\|_{X}\right)$ e $\left(E,\|\cdot\|_{E}\right)$ forem espaços de Banach e $\sigma: X \longrightarrow E$ for uma função uniformemente contínua e não colapsada, ${ }^{3}$ então, para todo $p \in[1,+\infty[$, existe $\omega_{p}: X \longrightarrow \ell_{p}\left(E,\|\cdot\|_{E}\right)^{4}$ tal que $\omega_{p}$ é, ao mesmo tempo, um mergulho uniforme e um mergulho grosseiro. Tendo em vista que, por definição, mergulhos uniformes são, trivialmente, não colapsados, e que, para todo $p \in\left[1,+\infty\left[, \ell_{p}\left(\ell_{p}\right)\right.\right.$ é isométrico a $\ell_{p}$ (em relação às normas usuais sobre $\ell_{p}\left(\ell_{p}\right)$ e $\ell_{p}$ ), esse resultado nos mostra que a resposta à questão acima é afirmativa (ao menos)

\footnotetext{
${ }^{2}$ Sendo $\mathbb{K}$ o corpo dos números reais ou o dos números complexos.

${ }^{3}$ Dizemos que uma função $\sigma: X \longrightarrow E$ é não colapsada se existirem $\Delta, M>0$ tais que $\|\sigma(x)-\sigma(y)\|_{E} \geq M$ para todos $x, y \in X$ tais que $\|x-y\|_{X} \geq \Delta$.

${ }^{4}$ Recorde que

$$
\ell_{p}\left(E,\|\cdot\|_{E}\right):=\left\{\left(x_{n}\right)_{n \in \mathbb{N}}: x_{n} \in E \forall n \in \mathbb{N} \text { e }\left(\left\|x_{n}\right\|_{E}\right)_{n \in \mathbb{N}} \in \ell_{p}\right\}
$$
}

é um espaço vetorial em relação às operações usuais de soma e de multiplicação por escalar de sequências e que 
no caso em que $E=\ell_{p}$ para algum $p \in[1,+\infty[$. E mais: ele nos diz que, nesse caso, existe um mergulho grosseiro de $\left(X,\|\cdot\|_{X}\right)$ em $\left(E,\|\cdot\|_{E}\right)$ o qual é, também, um mergulho uniforme.

Outro desses resultados nos diz que, se, em adição às hipóteses do teorema anterior, $\sigma$ for limitada, então existirá um mergulho grosseiro uniformemente contínuo de $\left(X,\|\cdot\|_{X}\right)$ em $\left(E \times E,\|\cdot\|_{E \times E}\right)$. Isso é particularmente útil em vários casos de interesse, dado que, em muitas situações, é preferível trabalhar com $\left(E \times E,\|\cdot\|_{E \times E}\right)$ em detrimento de $\left(\ell_{p}\left(E,\|\cdot\|_{E}\right),\|\cdot\|_{p}\right)$.

Por fim, é importante ressaltar que ambos os teoremas são consequências de resultados mais gerais e com ampla aplicabilidade, os quais não enunciaremos aqui. Tais resultados, todavia, são explorados e discutidos em detalhes no capítulo 5 .

Já no capítulo 6, abandonamos o terreno relativamente mapeado dos espaços de Banach e voltamos nossa atenção ao estudo de uma classe particular de estruturas coarse sobre grupos: a das estruturas coarse invariantes à esquerda, dentre as quais se destaca a estrutura coarse à esquerda de um grupo topológico. Um dos trabalhos pioneiros nessa área, e principal referência para esse capítulo, é o recente manuscrito de Christian Rosendal, intitulado Coarse Geometry of Topological Groups [Ros17a], cuja consulta recomendamos a quem quer que esteja interessado em se aprofundar no estudo das propriedades de larga escala dos grupos topológicos.

O capítulo encontra-se dividido em três diferentes seções. Na primeira delas, exploramos a relação entre o conjunto das estruturas coarse invariantes à esquerda sobre um grupo $(G, \cdot)$ e uma classe particular de ideais sobre $G$. O objetivo dessa seção é mostrar que é possível descrever uma estrutura coarse invariante à esquerda em $(G, \cdot)$ em termos de um certo ideal sobre $G$, e vice-versa. Na segunda seção, direcionamos o nosso foco para o estudo dos conjuntos grosseiramente limitados de um espaço coarse. ${ }^{5}$ Embora, em princípio, a noção de conjunto grosseiramente limitado estenda-se a um espaço coarse arbitrário, nosso principal interesse, nessa seção, é caracterizar os conjuntos grosseiramente limitados em relação à estrutura coarse à esquerda de um grupo topológico e, a partir disso e dos resultados da primeira parte, demonstrar que ela pode ser descrita em termos da coleção desses conjuntos. Esse é um resultado de caráter bastante geral, e, na terceira seção, discutimos uma de suas aplicações. Mais especificamente, provamos que, no caso particular do grupo aditivo de um espaço normado, a estrutura coarse à esquerda coincide com a estrutura coarse limitada induzida pela norma. E, com essa demonstração, encerramos o capítulo.

$\|\cdot\|_{p}: \ell_{p}\left(E,\|\cdot\|_{E}\right) \longrightarrow \mathbb{R}$ tal que

$$
\left\|\left(x_{n}\right)_{n \in \mathbb{N}}\right\|_{p}=\left(\sum_{n=1}^{+\infty}\left\|x_{n}\right\|_{E}^{p}\right)^{\frac{1}{p}}
$$

é uma norma sobre $\ell_{p}\left(E,\|\cdot\|_{E}\right)$.

${ }^{5}$ Dada uma estrutura coarse $\mathcal{E}$ sobre um conjunto não vazio $X$, dizemos que um subconjunto $A \subseteq X$ é grosseiramente limitado em $(X, \mathcal{E})$ se $A \times A \in \mathcal{E}$. 


\section{Capítulo 2}

\section{PRELIMINARES}

Neste capítulo, apresentaremos algumas definições relevantes e enunciaremos resultados importantes para o entendimento do restante do trabalho. No entanto, por se tratar de um conteúdo com o qual o leitor já está, ao menos em parte, familiarizado, os resultados aqui enunciados serão, salvo raras exceções, admitidos sem demonstração.

\subsection{NotaÇÃo}

Vamos começar estabelecendo a notação básica. Ao longo do texto, utilizaremos as notações $\mathbb{R}, \mathbb{C}, \mathbb{Z}$ e $\mathbb{Q}$ para designar, respectivamente, os conjuntos dos números reais, dos números complexos, dos números inteiros e dos números racionais. Algumas vezes, no entanto, faremos uso dos símbolos $\mathbb{R}$ e $\mathbb{C}$ para denotar, respectivamente, também os corpos dos números reais e dos números complexos. Apesar disso, em geral, o contexto será suficiente para esclarecer o significado preciso dos símbolos em questão, de modo que nenhuma confusão resultará desta pequena ambiguidade.

O conjunto

$$
\{1,2,3, \ldots\}
$$

será denotado por $\mathbb{N}$, e, para nos referirmos a $\mathbb{N} \cup\{0\}$, utilizaremos o símbolo $\mathbb{N}_{0}$. Vale ainda ressaltar que, salvo menção em contrário, sempre que fizermos referência a um corpo $\mathbb{K}$, estaremos assumindo, implicitamente, que $\mathbb{K}=\mathbb{R}$ ou $\mathbb{K}=\mathbb{C}$.

\subsection{RELAÇÕES BINÁRIAS SOBRE UM CONJUNTO}

Nesta seção, vamos introduzir o importante conceito de relação binária sobre um conjunto, bem como estabelecer notações e enunciar resultados os quais serão úteis ao longo dos próximos capítulos.

DeFinições 2.2.1. Seja $X$ um conjunto arbitrário. Uma relação binária sobre $X$ é um subconjunto de $X \times X$.

A relação binária $\{(x, x): x \in X\}$ é chamada diagonal de $X$ e será denotada por $\Delta(X)$. Quando o conjunto $X$ estiver claro pelo contexto, escreveremos apenas $\Delta$ para nos referirmos a $\Delta(X)$. 
Dada uma relação binária $E$ sobre $X$, a inversa de $E$ é a relação binária

$$
E^{-1}:=\{(x, y) \in X \times X:(y, x) \in E\} .
$$

Se $E=E^{-1}$, dizemos que $E$ é simétrica.

Dadas relações binárias $E$ e $F$ sobre $X$, a composição de $F$ com $E$ é a relação binária

$$
E \circ F:=\{(x, y) \in X \times X: \exists z \in X \text { t.q. }(x, z) \in F \text { e }(z, y) \in E\} .
$$

Note que a operação o é uma extensão natural da composição de funções. De fato, se $f$ e $g$ são funções de $X$ em $X$, é imediato ver que $\operatorname{Gr}(f \circ g)=\operatorname{Gr}(f) \circ \operatorname{Gr}(g)$.

OBSERVAÇÃo 2.2.2. Alguns livros (como, por exemplo, [Ros17a]) utilizam uma notação um pouco diferente para indicar a composição de relações binárias. Nessa notação, as posições de $E$ e $F$ em $E \circ F$ aparecem invertidas em relação à nossa convenção, sendo a composição de $F$ com $E$ denotada por $F \circ E$ (em substituição a $E \circ F$ ). Note que, nesse caso,

$$
E \circ F:=\{(x, y) \in X \times X: \exists z \in X \text { t.q. }(x, z) \in E \text { e }(z, y) \in F\} .
$$

A seguir, vamos listar algumas propriedades básicas referentes às operações com relações binárias.

Proposição 2.2.3. Dadas relações binárias $E, F$ e $G$ sobre um mesmo conjunto $X$, temos que:

a) $(E \circ F) \circ G=E \circ(F \circ G)$;

b) $(E \cup F)^{-1}=E^{-1} \cup F^{-1}$;

c) $(E \cap F)^{-1}=E^{-1} \cap F^{-1}$;

d) $(E \circ F)^{-1}=F^{-1} \circ E^{-1}$.

Demonstração. Vamos provar o item a). A demonstração dos demais itens fica a cargo do leitor.

Dado $(x, y) \in X \times X$, temos que

$$
(x, y) \in(E \circ F) \circ G \Leftrightarrow \exists z \in X \text { t.q. }(x, z) \in G \text { e }(z, y) \in E \circ F .
$$

E, para todo $z \in X$,

$$
(z, y) \in E \circ F \Leftrightarrow \exists z^{\prime} \in X \text { t.q. }\left(z, z^{\prime}\right) \in F \text { e }\left(z^{\prime}, y\right) \in E .
$$

Dessa forma,

$$
(x, y) \in(E \circ F) \circ G \Leftrightarrow \exists z, z^{\prime} \in X \text { t.q. }(x, z) \in G,\left(z, z^{\prime}\right) \in F \text { e }\left(z^{\prime}, y\right) \in E .
$$

Analogamente,

$$
\begin{aligned}
(x, y) \in E \circ(F \circ G) & \Leftrightarrow \exists z^{\prime} \in X \text { t.q. }\left(x, z^{\prime}\right) \in F \circ G \text { e }\left(z^{\prime}, y\right) \in E \\
& \Leftrightarrow \exists z, z^{\prime} \in X \text { t.q. }(x, z) \in G,\left(z, z^{\prime}\right) \in F \text { e }\left(z^{\prime}, y\right) \in E .
\end{aligned}
$$

Comparando $(*)$ e $(* *)$, concluímos que

$$
(x, y) \in E \circ(F \circ G) \Leftrightarrow(x, y) \in(E \circ F) \circ G .
$$


E, sendo $(x, y) \in X \times X$ um ponto arbitrário, disso resulta que $(E \circ F) \circ G=E \circ(F \circ G)$.

Encerraremos esta discussão enunciando uma importante propriedade da composição de relações, a qual será utilizada mais adiante.

Proposição 2.2.4. Para todo $n \in \mathbb{N}$ e quaisquer relações binárias $E_{1}, \ldots, E_{n}$ sobre um conjunto arbitrário $X$, vale que $\left(E_{1} \circ \ldots \circ E_{n}\right) \cup\left(F_{1} \circ \ldots \circ F_{n}\right) \subseteq\left(E_{1} \cup F_{1}\right) \circ \ldots \circ\left(E_{n} \cup F_{n}\right)$.

Demonstração. Vamos provar isso por indução sobre $n$. A propriedade é óbvia se $n=1$. Mostremos que ela vale para $n=2$. Com efeito, note que, para todo $(x, y) \in X \times X$,

$$
\begin{gathered}
(x, y) \in\left(E_{1} \circ E_{2}\right) \cup\left(F_{1} \circ F_{2}\right) \Rightarrow\left[(x, y) \in E_{1} \circ E_{2} \text { ou }(x, y) \in F_{1} \circ F_{2}\right] \Rightarrow \\
{\left[\exists z \in X \text { t.q. }(x, z) \in E_{2} \text { e }(z, y) \in E_{1}\right] \text { ou }\left[\exists z \in X \text { t.q. }(x, z) \in F_{2} \text { e }(z, y) \in F_{1}\right] \Rightarrow} \\
\exists z \in X \text { t.q. }\left[(x, z) \in E_{2} \text { e }(z, y) \in E_{1}\right] \text { ou }\left[(x, z) \in F_{2} \text { e }(z, y) \in F_{1}\right] \Rightarrow \\
\exists z \in X \text { t.q. }(x, z) \in E_{2} \cup F_{2} \text { e }(z, y) \in E_{1} \cup F_{1} \Rightarrow(x, y) \in\left(E_{1} \cup F_{1}\right) \circ\left(E_{2} \cup F_{2}\right) .
\end{gathered}
$$

Disso concluímos que $\left(E_{1} \circ E_{2}\right) \cup\left(F_{1} \circ F_{2}\right) \subseteq\left(E_{1} \cup F_{1}\right) \circ\left(E_{2} \cup F_{2}\right)$.

Suponhamos, agora, que essa propriedade valha para algum $n \in\{2,3, \ldots\}$, e mostremos que ela vale para $n+1$. Observe que, pela associatividade de $\circ$ e pelo caso $n=2$, temos que

$$
\begin{aligned}
\left(E_{1} \circ \ldots \circ E_{n+1}\right) \cup\left(F_{1} \circ \ldots \circ F_{n+1}\right) & =\left[\left(E_{1} \circ \ldots \circ E_{n}\right) \circ E_{n+1}\right] \cup\left[\left(F_{1} \circ \ldots \circ F_{n}\right) \circ F_{n+1}\right] \\
& \subseteq\left[\left(E_{1} \circ \ldots \circ E_{n}\right) \cup\left(F_{1} \circ \ldots \circ F_{n}\right)\right] \circ\left(E_{n+1} \cup F_{n+1}\right) .
\end{aligned}
$$

Mas, pela hipótese de indução,

$$
\left(E_{1} \circ \ldots \circ E_{n}\right) \cup\left(F_{1} \circ \ldots \circ F_{n}\right) \subseteq\left(E_{1} \cup F_{1}\right) \circ \ldots \circ\left(E_{n} \cup F_{n}\right) .
$$

Logo:

$$
\begin{aligned}
\left(E_{1} \circ \ldots \circ E_{n+1}\right) \cup\left(F_{1} \circ \ldots \circ F_{n+1}\right) & \subseteq\left[\left(E_{1} \cup F_{1}\right) \circ \ldots \circ\left(E_{n} \cup F_{n}\right)\right] \circ\left(E_{n+1} \cup F_{n+1}\right) \\
& =\left(E_{1} \cup F_{1}\right) \circ \ldots \circ\left(E_{n+1} \cup F_{n+1}\right) .
\end{aligned}
$$

(Note que utilizamos novamente a associatividade de o na última igualdade.)

\subsection{EsPAÇOS MÉTRICOS E PSEUdomÉtricos}

Esta seção tem como objetivo recordar conceitos básicos da teoria dos espaços métricos e pseudométricos e estabelecer notações convenientes, as quais utilizaremos recorrentemente ao longo do trabalho.

\section{PRimeiros CONCEITOS}

Vamos começar recordando as definições de métrica e pseudométrica.

DefiniçÃo 2.3.1 (PSEudométrica). Seja $X$ um conjunto não vazio. Uma pseudométrica sobre $X$ é uma função $d: X \times X \longrightarrow \mathbb{R}$ tal que, para quaisquer $x, y, z \in X$, tenhamos

a) $d(x, y) \geq 0$ 
b) $d(x, x)=0$;

c) $d(x, y)=d(y, x)$;

d) $d(x, y) \leq d(x, z)+d(z, y)$.

Se, além disso, valer que, para todos $x, y \in X$,

$$
d(x, y)=0 \Rightarrow x=y,
$$

dizemos que $d$ é uma métrica sobre $X$.

DEFINIÇÃo 2.3.2 (ESPAÇO PSEUdOMÉTRICO). Um espaço pseudométrico é um par $(X, d)$, em que $X$ é um conjunto não vazio e $d$ é uma pseudométrica sobre $X$. Se, além disso, $d$ for uma métrica sobre $X$, diremos que $(X, d)$ é um espaço métrico.

Exemplos 2.3.3. Seja $X$ um conjunto não vazio.

a) A pseudométrica trivial sobre $X$ é a função $d: X \times X \longrightarrow \mathbb{R}$ tal que $d(x, y)=0$ para todos $x, y \in X$.

b) A função $d: X \times X \longrightarrow \mathbb{R}$ tal que

$$
d(x, y)= \begin{cases}0, & \text { se } x=y \\ 1, & \text { se } x \neq y\end{cases}
$$

é uma métrica sobre $X$, a qual denominamos métrica discreta.

c) A métrica usual sobre $\mathbb{K}$ é a função $d: \mathbb{K} \times \mathbb{K} \longrightarrow \mathbb{R}$ tal que

$$
d(x, y)=|x-y|
$$

A seguir, vamos introduzir um pouco da terminologia usual no contexto dos espaços pseudométricos.

DeFiniÇÃo 2.3.4 (BOla ABERTA E BOla FECHADA). Dados um espaço pseudométrico $(X, d)$, um real $r>0$ e um ponto $x_{0} \in X$, chamamos de bola aberta de centro $x_{0}$ e raio $r$ ao conjunto

$$
B_{X}^{d}\left(x_{0}, r\right):=\left\{x \in X: d\left(x, x_{0}\right)<r\right\} .
$$

Analogamente, o conjunto

$$
B_{X}^{d}\left[x_{0}, r\right]:=\left\{x \in X: d\left(x, x_{0}\right) \leq r\right\}
$$

denomina-se bola fechada de centro $x_{0}$ e raio $r$. Quando o conjunto $X$ e a pseudométrica $d$ estiverem claros pelo contexto, escreveremos apenas $B\left(x_{0}, r\right)$ para nos referirmos à bola aberta de centro $x_{0}$ e raio $r$. Uma observação semelhante vale para a bola fechada.

DeFINIÇÃo 2.3.5. Dados um espaço pseudométrico $(X, d)$ e um subconjunto não vazio $A \subseteq X$, definimos o diâmetro de $A$ em relação a d como sendo o

$$
\sup \{d(x, y): x, y \in A\} .
$$


NotaÇÃo: $\mathrm{O}$ diâmetro de um conjunto $A \subseteq X$ em um espaço pseudométrico $(X, d)$ será denotado por $\operatorname{diam}_{d}(A)$.

A proposição a seguir estabelece uma caracterização para os conjuntos de diâmetro finito em um espaço pseudométrico.

Proposição 2.3.6. Sejam $(X, d)$ um espaço pseudométrico e $A \subseteq X$ um subconjunto qualquer. Nessas condições, $\operatorname{diam}_{d}(A)$ é finito se, e somente se, para cada $x \in X$, existe $M_{x} \geq 0$ tal que $d(x, y) \leq M_{x}$ para todo $y \in A$.

Demonstração. $(\Rightarrow)$ Se $A=\emptyset$, isso é óbvio. Caso contrário, basta tomar $x_{0} \in A$ qualquer e notar que, dado $x \in X$, temos que

$$
d(x, y) \leq d\left(x, x_{0}\right)+d\left(x_{0}, y\right) \leq \underbrace{d\left(x, x_{0}\right)+\operatorname{diam}_{d}(A)}_{M_{x}}
$$

para todo $y \in A$.

$(\Leftarrow)$ Sejam $x_{0} \in X$ e $M_{x_{0}} \geq 0$ tais que $d\left(x_{0}, y\right) \leq M_{x_{0}}$ para todo $y \in A$. Dados $x, y \in A$, observe que

$$
d(x, y) \leq d\left(x, x_{0}\right)+d\left(x_{0}, y\right) \leq M_{x_{0}}+M_{x_{0}}=2 M_{x_{0}} .
$$

Como $x, y \in A$ são arbitrários, disso concluímos que $\operatorname{diam}_{d}(A) \leq 2 M_{x_{0}}$ e, em particular, é finito.

\section{ESPAÇOS MÉTRICOS COMPLETOS}

A seguir, faremos algumas definições e comentários a respeito de sequências em espaços métricos. Comecemos com as seguintes definições:

DefiniçÃo 2.3.7 (CONVERGÊnCIA). Dado um espaço métrico $(X, d)$, dizemos que uma sequência $\left(x_{n}\right)_{n \in \mathbb{N}}$ de elementos de $X$ converge em $(X, d)$ se existir $x \in X$ tal que, para todo $\varepsilon>0$, exista $N_{0} \in \mathbb{N}$ de modo que, para todo $n \in \mathbb{N}$,

$$
n>N_{0} \Rightarrow d\left(x_{n}, x\right)<\varepsilon .
$$

NotaÇÃo: Dados um espaço métrico $(X, d)$ e um ponto $x \in X$, utilizaremos as notações $x_{n} \longrightarrow x$ e $\lim _{n \rightarrow+\infty} x_{n}=x$ para indicar que uma sequência $\left(x_{n}\right)_{n \in \mathbb{N}}$ em $(X, d)$ converge a $x$

DefiniçÃo 2.3.8 (SEquÊnCIA Limitada). Dado um espaço métrico $(X, d)$, dizemos que uma sequência $\left(x_{n}\right)_{n \in \mathbb{N}}$ de elementos de $X$ é limitada em $(X, d)$ se o conjunto $\left\{x_{n}: n \in \mathbb{N}\right\}$ possuir diâmetro finito em relação a $d$.

OBSERVAÇÃo 2.3.9. Toda sequência convergente é limitada.

Uma importante subclasse de sequências em um espaço métrico é a das sequências de Cauchy. Formalmente, temos a sequinte definição:

Definição 2.3.10 (SEquênCia De CAuChy). Dado um espaço métrico $(X, d)$, dizemos que uma sequência $\left(x_{n}\right)_{n \in \mathbb{N}}$ de elementos de $X$ é de Cauchy se, para todo $\varepsilon>0$, existir $N_{0} \in \mathbb{N}$ tal que, para todos $n, m \in \mathbb{N}$,

$$
n, m>N_{0} \Rightarrow d\left(x_{n}, x_{m}\right)<\varepsilon .
$$


É fácil ver que toda sequência convergente em um espaço métrico $(X, d)$ é de Cauchy em $(X, d)$. No entanto, a recíproca desse resultado nem sempre é verdadeira. Os espaços métricos para os quais isso vale são chamados completos. O estudo dos espaços métricos completos é um tópico central em Análise Funcional. E, assim sendo, vale a pena apresentarmos o conceito de completude em forma de definição.

DeFiniÇÃo 2.3.11 (EsPaÇO MÉtrico COMPleto). Um espaço métrico $(X, d)$ é completo se toda sequência de Cauchy em $(X, d)$ convergir em $(X, d)$.

Exemplos 2.3.12.

a) Um espaço métrico $(X, d)$, em que $d$ é a métrica discreta, é, trivialmente, completo. Com efeito, dada uma sequência $\left(x_{n}\right)_{n \in \mathbb{N}}$ de Cauchy em $(X, d)$, podemos fixar $N_{0} \in \mathbb{N}$ tal que, para todos $n, m \in \mathbb{N}$,

$$
n, m \geq N_{0} \Rightarrow d\left(x_{n}, x_{m}\right)<1 \Rightarrow d\left(x_{n}, x_{m}\right)=0 \Rightarrow x_{n}=x_{m},
$$

do que segue que $\lim _{n \rightarrow+\infty} x_{n}=x_{N_{0}}$.

b) $\mathrm{O}$ conjunto $\mathbb{K}$ é completo em relação à métrica usual.

c) O espaço métrico $(\mathbb{Q}, d)$, em que

$$
d(x, y):=|x-y| \quad \forall x, y \in \mathbb{Q},
$$

não é completo. Com efeito, toda sequência $\left(x_{n}\right)_{n \in \mathbb{N}}$ de racionais tal que $0 \leq x_{n}<2$ para todo $n \in \mathbb{N}$ e $\lim _{n \rightarrow+\infty} x_{n}{ }^{2}=2$ é de Cauchy em $(\mathbb{Q}, d)$, mas não tem limite em $(\mathbb{Q}, d)$.

\section{Continuidade E CONTINUIDADE UNIFORME}

Uma das principais motivações para o estudo dos espaços pseudométricos reside no fato de que eles nos permitem generalizar vários aspectos da geometria do $\mathbb{R}^{n}$. Isso inclui, em particular, os conceitos de continuidade e de continuidade uniforme, os quais discutiremos rapidamente a seguir.

Definição 2.3.13 (Continuidade). Dados espaços pseudométricos $(x, d)$ e $(Y, \partial)$, dizemos que uma função $f: X \longrightarrow Y$ é contínua em um ponto $x \in X$ se, para todo $\varepsilon>0$, existir $\delta>0$ tal que, para todos $x, y \in X$,

$$
d(x, y)<\delta \Rightarrow \partial(f(x), f(y))<\varepsilon .
$$

Se $f$ for contínua em $x$ para todo $x \in X$, diremos, apenas, que $f$ é contínua.

Exemplo 2.3.14. Sejam $(X, d)$ e $(Y, \partial)$ espaços pseudométricos. Nessas condições, se $d$ for a métrica discreta ou $\partial$ for a pseudométrica trivial, toda função $f: X \longrightarrow Y$ será contínua.

Sejam $(x, d)$ e $(Y, \partial)$ espaços pseudométricos e $f: X \longrightarrow Y$ uma função contínua. Observe que, nesse caso, segue da definição 2.3 .13 que, para cada $\varepsilon>0$ e cada $x \in X$, podemos encontrar $\delta>0$ tal que $\partial(f(x), f(y))<\varepsilon$ para todos $x, y \in X$ tais que $d(x, y)<\delta$. Note, no entanto, que um tal $\delta$ depende, a princípio, tanto de $x$ quanto de $\varepsilon$. Será que, nessas condições, é possível obter um $\delta>0$ que dependa de $\varepsilon$, mas não de $x$ ? As funções com tal propriedade são chamadas uniformemente contínuas. A definição a seguir formaliza o que foi dito: 
Definição 2.3.15 (Continuidade uniforme). Dados espaços pseudométricos $(X, d)$ e $(Y, \partial)$, dizemos que uma função $f: X \longrightarrow Y$ é uniformemente contínua se, para cada $\varepsilon>0$, existir $\delta>0$ tal que, para todos $x, y \in X$,

$$
d(x, y)<\delta \Rightarrow \partial(f(x), f(y))<\varepsilon .
$$

OBSERVAÇÃo 2.3.16. Evidentemente, toda função uniformemente contínua é, também, continua. No entanto, existem funções, como, por exemplo, $f: \mathbb{R} \longrightarrow \mathbb{R}$ tal que $f(x)=x^{2}$, as quais são contínuas, mas não uniformemente contínuas.

\section{CONVERGÊNCIA UNIFORME}

Um conceito marginal para este trabalho, embora, de modo geral, bastante importante em Análise, é o de convergência uniforme de uma sequência de funções em um espaço pseudométrico. Por essa razão, embora façamos referência à convergência uniforme, ao longo do trabalho, apenas uma vez, vale a pena enunciar esse conceito em forma de definição.

DefiniçÃo 2.3.17 (CONVERGÊnCIA Uniforme). Sejam $(X, d)$ um espaço pseudométrico e $S$ um conjunto não vazio, e, para cada $n \in \mathbb{N}$, seja $f_{n}: S \longrightarrow X$ uma função. Nessas condições, dizemos que a sequência $\left(f_{n}\right)_{n \in \mathbb{N}}$ converge uniformemente a uma função $f: S \longrightarrow X$ se, para cada $\varepsilon>0$, existir $N_{0} \in \mathbb{N}$ tal que, para todo $n \in \mathbb{N}$,

$$
n>N_{0} \Rightarrow d\left(f_{n}(s), f(s)\right)<\varepsilon \quad \forall s \in S .
$$

\subsection{SEQUÊNCIAS E SÉRIES DE NÚMEROS REAIS}

Nesta seção, vamos listar alguns resultados básicos sobre sequências e séries de números reais, os quais são usualmente estudados nos cursos de Cálculo ou em um primeiro curso de Análise Real.

Comecemos recordando um resultado clássico sobre sequências monótonas.

Proposição 2.4.1. Toda sequência monótona e limitada de números reais é convergente. Além disso, se $\left(a_{n}\right)_{n \in \mathbb{N}}$ for uma sequência nessas condições, então

$$
\lim _{n \rightarrow+\infty} a_{n}=\sup \left\{a_{n}: n \in \mathbb{N}\right\}
$$

se $\left(a_{n}\right)_{n \in \mathbb{N}}$ for crescente, $e$

$$
\lim _{n \rightarrow+\infty} a_{n}=\inf \left\{a_{n}: n \in \mathbb{N}\right\}
$$

se for decrescente.

A proposição 2.4.1 tem o seguinte corolário:

Corolário 2.4.2. Seja $\left(a_{n}\right)_{n \in \mathbb{N}}$ uma sequência de números reais não negativos. Nessas condições, se existir um número real $M>0$ tal que $\sum_{j=1}^{n} a_{j} \leq M$ para todo $n \in \mathbb{N}$, então $\sum_{n=1}^{+\infty} a_{n}$ converge, e a sua soma é menor ou igual a $M$. 
E, por sua vez, o corolário 2.4.2 pode ser utilizado para demonstrar um importante critério de convergência para séries de termos positivos, o qual denominamos Critério da comparação.

Proposição 2.4.3 (CRItério da COMPARAÇÃo). Sejam $\left(a_{n}\right)_{n \in \mathbb{N}} e\left(b_{n}\right)_{n \in \mathbb{N}}$ sequências de números reais não negativos, e suponhamos que existam $N_{0} \in \mathbb{N}$ e $M>0$ tais que $a_{n} \leq M b_{n}$ para todo $n \in \mathbb{N}$ tal que $n \geq N_{0}$. Nessas condições:

(a) Se $\sum_{n=1}^{+\infty} b_{n}$ convergir, então $\sum_{n=1}^{+\infty} a_{n}$ converge, e, nesse caso, $\sum_{n=N_{0}}^{+\infty} a_{n} \leq M \sum_{n=N_{0}}^{+\infty} b_{n}$;

(b) Se $\sum_{n=1}^{+\infty} a_{n}$ divergir, então $\sum_{n=1}^{+\infty} b_{n}$ diverge.

\subsection{ESPAÇOS VETORIAIS E TRANSFORMAÇÕES LINEARES}

A seguir, vamos recordar, rapidamente, as noções de álgebra linear necessárias para a compreensão dos demais capítulos. Espera-se, no entanto, que o leitor já tenha grande familiaridade com os temas aqui discutidos. Excepcionalmente ao longo desta seção, o símbolo $\mathbb{K}$ denotará, quase sempre, um corpo qualquer.

Vamos começar com a definição de espaço vetorial.

DEFINIÇÃo 2.5.1 (ESPAÇO VETORIAL). Um espaço vetorial sobre um corpo $\mathbb{K}$ (também chamado um $\mathbb{K}$ - espaço vetorial) é uma tripla $(V,+, \cdot)$, em que $V$ é um conjunto não vazio e

$$
\begin{aligned}
+: V \times V & \longrightarrow V \\
(u, v) & \longmapsto u+v, \\
\cdot: \mathbb{K} \times V & \longrightarrow V \\
(\lambda, v) & \longmapsto \lambda \cdot v
\end{aligned}
$$

são operações binárias, denominadas, respectivamente, adição (ou soma) e multiplicação por escalar, com as seguintes propriedades:

$\left(A_{1}\right)(u+v)+w=u+(v+w)$ para todos $u, v, w \in V$

$\left(A_{2}\right) u+v=v+u$ para todos $u, v \in V ;$

$\left(A_{3}\right)$ Existe um único $\overrightarrow{0} \in V$, o qual denotaremos por $0_{V}$, tal que $v+\overrightarrow{0}=v$ para todo $v \in V$;

$\left(A_{4}\right)$ Para cada $u \in V$, existe $v \in V$ tal que $u+v=0_{V}$;

$\left(M_{1}\right) \lambda \cdot(\mu \cdot v)=(\lambda \mu) \cdot v$ para todos $\lambda, \mu \in \mathbb{K}$ e todo $v \in V$

$\left(M_{2}\right) 1 \cdot v=v$ para todo $v \in V ; \quad($ em que $1 \in \mathbb{K}$ é tal que $1 \lambda=\lambda$ para todo $\lambda \in \mathbb{K}$ )

$\left(D_{1}\right) \lambda \cdot(u+v)=\lambda \cdot u+\lambda \cdot v$ para todos $u, v \in V$ e todo $\lambda \in \mathbb{K} ;$ 
$\left(D_{2}\right) \underbrace{(\lambda+\mu)}_{\text {soma em } \mathbb{K}} \cdot v=\underbrace{\lambda \cdot v+\mu \cdot v}_{\text {soma em } V}$ para todos $\lambda, \mu \in \mathbb{K}$ e todo $v \in V$.

Em geral, quando as operações $+\mathrm{e} \cdot$ estiverem claras pelo contexto, escreveremos apenas $V$ para nos referirmos a $(V,+, \cdot)$.

OBSERVAÇÃo 2.5.2. Seja $V$ um espaço vetorial sobre um corpo $\mathbb{K}$.

a) Os elementos de $V$ são, também, denominados vetores de $V$. (Assim sendo, um vetor nada mais é do que um elemento de um espaço vetorial.)

b) O elemento $0_{V}$, definido em $\left(A_{3}\right)$, é denominado vetor nulo de $V$, e é o elemento neutro da adição em $V$.

c) Para cada $u \in V$, o elemento $v \in V$ tal que $u+v=0_{V}$ é único, e será denotado por $-u$. Dado $u \in V$, dizemos que $-u$ é o oposto de $u$ em $V$.

NotAÇÃo: Com frequência, escreveremos apenas $\lambda v$ para nos referirmos a $\lambda \cdot v$.

Exemplos 2.5.3. Nos itens c), d) e e), consideremos $\mathbb{K}$ igual a $\mathbb{R}$ ou $\mathbb{C}$.

a) Para todo $n \in \mathbb{N}$ e todo corpo $\mathbb{K}$, o conjunto

$$
\mathbb{K}^{n}:=\left\{\left(x_{1}, \ldots, x_{n}\right): x_{i} \in \mathbb{K} \forall i \in\{1, \ldots, n\}\right\},
$$

é um espaço vetorial sobre $\mathbb{K}$ em relação às operações usuais de adição e de multiplicação por escalar.

b) Para todo corpo $\mathbb{K}$, o conjunto

$$
\mathbb{K}^{\mathbb{N}}:=\left\{\left(x_{n}\right)_{n \in \mathbb{N}}: x_{n} \in \mathbb{K} \forall n \in \mathbb{N}\right\}
$$

é um espaço vetorial sobre $\mathbb{K}$ em relação às operações usuais de soma e de multiplicação por escalar de sequências.

c) Para cada $p \in[1,+\infty[$, o conjunto

$$
\ell_{p}:=\left\{\left(x_{n}\right)_{n \in \mathbb{N}} \in \mathbb{K}^{\mathbb{N}}: \sum_{n=1}^{+\infty}\left|x_{n}\right|^{p} \text { converge }\right\}
$$

é um espaço vetorial sobre $\mathbb{K}$ em relação às operações usuais de adição e de multiplicação por escalar.

d) O conjunto

$$
\ell_{\infty}:=\left\{\left(x_{n}\right)_{n \in \mathbb{N}} \in \mathbb{K}^{\mathbb{N}}:\left(x_{n}\right)_{n \in \mathbb{N}} \text { é limitada }\right\}
$$

é um espaço vetorial em relação às operações usuais de soma e de multiplicação por escalar.

e) O conjunto

$$
c_{0}:=\left\{\left(x_{n}\right)_{n \in \mathbb{N}} \in \mathbb{K}^{\mathbb{N}}:\left(x_{n}\right)_{n \in \mathbb{N}} \text { converge a } 0\right\}
$$

é um espaço vetorial em relação às operações usuais. Observe que $c_{0} \subseteq \ell_{\infty}$, uma vez que toda sequência convergente é limitada. 
Definição 2.5.4 (COnjunto Gerador). Dado um $\mathbb{K}$ - espaço vetorial $V$, dizemos que um subconjunto $\mathscr{B} \subseteq V$ é um conjunto gerador de $V$ (ou que $\mathscr{B}$ gera $V$ ) se, para cada $v \in V$, existirem $n \in \mathbb{N}, \lambda_{1}, \ldots, \lambda_{n} \in \mathbb{K}$ e $v_{1}, \ldots, v_{n} \in \mathscr{B}$ tais que

$$
v=\lambda_{1} v_{1}+\ldots+\lambda_{n} v_{n}
$$

Se $V$ admitir um conjunto gerador finito, diremos que $V$ é finitamente gerado. Caso contrário, diremos que $V$ possui dimensão infinita.

OBSERVAÇÃo 2.5.5. Todo $\mathbb{K}$ - espaço vetorial admite, ao menos, um conjunto gerador. Com efeito, dado um $\mathbb{K}$ - espaço vetorial $V$, é imediato constatar que $V$ é, ele próprio, um conjunto gerador de $V$.

DeFiniÇÃo 2.5.6 (SubespaÇO VEtorial). Sejam $V$ um espaço vetorial sobre um corpo $\mathbb{K}$ e $W \subseteq V$ um subconjunto não vazio. Nessas condições, dizemos que $W$ é um subespaço vetorial de $V$ se $W$ possuir as seguintes propriedades:

a) $0_{V} \in W$

b) Se $u, v \in W$, então $u+v \in W$;

c) Se $u \in W$ e $\lambda \in \mathbb{K}$, então $\lambda \cdot v \in W$.

OBSERVAÇÃo 2.5.7. Todo subespaço vetorial $W$ de um $\mathbb{K}$ - espaço vetorial $(V,+, \cdot)$ é, ele próprio, um $\mathbb{K}$ - espaço vetorial (em relação a $+1_{W \times W} \mathrm{e} \cdot 1_{\mathbb{K} \times W}$ ).

EXEMPLOS 2.5.8.

a) Para todo $p \in[1,+\infty], \ell_{p}$ é um subesbaço vetorial de $\mathbb{K}^{\mathbb{N}}$ em relação às operações usuais de soma e de multiplicação por escalar de sequências.

b) $c_{0}$ é um subespaço vetorial de $\ell_{\infty}$.

c) Dados um espaço vetorial $V$ sobre um corpo $\mathbb{K}$ e um subconjunto não vazio $\mathcal{S} \subseteq V$, não é difícil mostrar que o conjunto

$$
W_{\mathcal{S}}:=\left\{\sum_{i=1}^{n} \alpha_{i} v_{i}: n \in \mathbb{N}, \alpha_{1}, \ldots, \alpha_{n} \in \mathbb{K} \text { e } v_{1}, \ldots, v_{n} \in \mathcal{S}\right\}
$$

é um subespaço vetorial. Dizemos que $W_{\mathcal{S}}$ é o subespaço de $V$ gerado por $\mathcal{S}$.

[No caso em que $\mathcal{S}=\emptyset$, definimos $W_{\mathcal{S}}:=\left\{0_{V}\right\}$ (por convenção).]

Uma vez estabelecidas as definições básicas, podemos, agora, voltar nossa atenção aos morfismos entre $\mathbb{K}$ - espaços vetoriais, também chamados transformações lineares, os quais nada mais são do que funções entre espaços vetoriais que preservam as operações de soma e de multiplicação por escalar dos espaços em questão.

DefiniÇÃo 2.5.9 (TRAnsformaÇÃo linear). Sejam $U$ e $V$ espaços vetoriais sobre um mesmo corpo $\mathbb{K}$. Uma transformação linear de $U$ em $V$ é uma função $T: U \longrightarrow V$ tal que

$$
T(u+\lambda \cdot v)=T(u)+\lambda \cdot T(v)
$$

para todos $u, v \in U$ e todo $\lambda \in \mathbb{K}$.

No caso especial em que $U=V$, dizemos que $T$ é um operador linear. 
OBSERVAÇÃo 2.5.10. Dados espaços vetoriais $U$ e $V$ sobre um mesmo corpo $\mathbb{K}$ e uma transformação linear $T: U \longrightarrow V$, observe que:

(a) $T\left(0_{U}\right)=0_{V}$;

(b) $T(-u)=-T(u)$ para todo $u \in U$.

A seguir, definiremos o conceito de núcleo de uma transformação linear.

DEFINIÇÃo 2.5.11. Dados espaços vetoriais $U$ e $V$ sobre um corpo $\mathbb{K}$ e uma transformação linear $T: U \longrightarrow V$, chamamos de núcleo de $T$ ao conjunto

$$
\operatorname{Ker}(T):=\left\{u \in U: T(u)=0_{V}\right\} .
$$

No que resta desta seção, vamos nos concentrar em um tipo especial de operador linear: as projeções.

DefiniçÃo 2.5.12 (ProjeÇÃo). Sejam $V$ um espaço vetorial sobre um corpo $\mathbb{K}$ e $W \subseteq V$ um subespaço de $V$. Dizemos que um operador linear $\pi: V \longrightarrow V$ é uma projeção sobre $W$ se $\operatorname{Im}(\pi) \subseteq W$ e $\pi(w)=w$ para cada $w \in W$.

ObSERVAÇÃo 2.5.13. Se $V$ é um espaço vetorial sobre um corpo $\mathbb{K}$ e $P: V \longrightarrow V$ é uma projeção sobre um subespaço $W$ de $V$, então $\operatorname{Im}(P)=W$. Com efeito, sendo $P$ uma projeção sobre $W$, temos, por hipótese, que $\operatorname{Im}(P) \subseteq W$. E, como $P(w)=w$ para todo $w \in W$, vale, também, a outra inclusão.

Vejamos, a seguir, um exemplo de projeção.

Exemplo 2.5.14. Para cada $n \in \mathbb{N}$, a função

$$
\begin{aligned}
& \pi_{n}: \mathbb{K}^{\mathbb{N}} \longrightarrow \mathbb{K}^{\mathbb{N}} \\
& \left(x_{j}\right)_{j \in \mathbb{N}} \longmapsto\left(x_{n} \delta_{j n}\right)_{j \in \mathbb{N}}
\end{aligned}
$$

é uma projeção sobre o subespaço de $\mathbb{K}^{\mathbb{N}}$ gerado por $\left\{\left(\delta_{j n}\right)_{j \in \mathbb{N}}\right\}$.

\subsection{ESPAÇOS NORMADOS}

Assim como o conceito de métrica, o conceito de norma surgiu a partir de uma tentativa de transportar certas noções e propriedades da geometria do $\mathbb{R}^{n}$ a conjuntos de funções e outros conjuntos abstratos. Nesta seção, vamos recordar, brevemente, definições e conceitos básicos da teoria dos espaços normados.

Deste ponto em diante, $\mathbb{K}$ denotará, novamente, sempre $\mathbb{R}$ ou $\mathbb{C}$.

DefiniçÃo 2.6.1 (Norma). Seja $X$ um espaço vetorial sobre um corpo $\mathbb{K}$. Uma norma sobre $X$ é uma função $\|\cdot\|: X \longrightarrow \mathbb{R}$ tal que, para todos $x, y \in X$ e todo $\lambda \in \mathbb{K}$ :

(1) $\|x\| \geq 0$

(2) $\|x\|=0 \Leftrightarrow x=0_{X}$;

(3) $\|\lambda \cdot x\|=|\lambda| \cdot\|x\|$; 
(4) $\|x+y\| \leq\|x\|+\|y\|$.

Dada uma norma $\|\cdot\|$ sobre $X$, dizemos que o par $(X,\|\cdot\|)$ é um espaço vetorial normado ou, simplesmente, um espaço normado.

OBSERVAÇÃo 2.6.2. Ao longo do trabalho, sempre que nada for dito em contrário, assumiremos, implicitamente, que estamos lidando com espaços normados sobre $\mathbb{K}$, em que, como sempre, $\mathbb{K}=\mathbb{R}$ ou $\mathbb{K}=\mathbb{C}$.

Vejamos, a seguir, alguns exemplos de espaços normados.

EXEMPLOS 2.6.3.

a) Para todo $n \in \mathbb{N}$, as funções $\|\cdot\|_{1},\|\cdot\|_{2},\|\cdot\|_{\max }: \mathbb{K}^{n} \longrightarrow \mathbb{R}$ tais que

$$
\begin{gathered}
\left\|\left(x_{1}, \ldots, x_{n}\right)\right\|_{1}=\sum_{i=1}^{n}\left|x_{i}\right| \\
\left\|\left(x_{1}, \ldots, x_{n}\right)\right\|_{2}=\left(\sum_{i=1}^{n}\left|x_{i}\right|^{2}\right)^{\frac{1}{2}}
\end{gathered}
$$

$\mathrm{e}$

$$
\left\|\left(x_{1}, \ldots, x_{n}\right)\right\|_{\max }=\max \left\{\left|x_{1}\right|, \ldots,\left|x_{n}\right|\right\}
$$

são normas sobre $\mathbb{K}^{n}$.

b) Para todo $p \in\left[1,+\infty\left[\right.\right.$, a função $|\cdot|_{p}: \ell_{p} \longrightarrow \mathbb{R}$ tal que

$$
\left|\left(x_{n}\right)_{n \in \mathbb{N}}\right|_{p}=\left(\sum_{n=1}^{+\infty}\left|x_{n}\right|^{p}\right)^{\frac{1}{p}}
$$

é uma norma em $\ell_{p}$.

c) A função $|\cdot|_{\infty}: \ell_{\infty} \longrightarrow \mathbb{R}$ tal que

$$
\left|\left(x_{n}\right)_{n \in \mathbb{N}}\right|_{\infty}=\sup \left\{\left|x_{n}\right|: n \in \mathbb{N}\right\}
$$

é uma norma em $\ell_{\infty}$, e a restrição de $|\cdot|_{\infty}$ a $c_{0}$ é uma norma sobre $c_{0}$.

OBSERVAÇÃo 2.6.4. Uma norma sobre um conjunto $X$ induz, naturalmente, uma métrica sobre $X$. Com efeito, dada uma norma $\|\cdot\|_{X}$ sobre $X$, não é difícil mostrar que $d: X \times X \longrightarrow \mathbb{R}$ tal que

$$
d(x, y):=\|x-y\|_{X}
$$

é uma métrica sobre $X$.

NotAÇÃo: A métrica induzida por uma norma $\|\cdot\|$ sobre um conjunto $X$ será denotada por $d_{\|\cdot\| \cdot}$

A observação 2.6.4 mostra que podemos enxergar um espaço normado $\left(E,\|\cdot\|_{E}\right)$ como espaço métrico. Tal espaço métrico pode ou não ser completo. Em caso afirmativo, dizemos que $\left(E,\|\cdot\|_{E}\right)$ é um espaço de Banach.

DeFiniÇÃo 2.6.5 (ESPAÇO DE BAnACH). Um espaço de Banach é um espaço vetorial normado e completo em relação à métrica induzida pela norma. 
Exemplo 2.6.6. Os espaços $\left(c_{0},|\cdot|_{\infty}\right),\left(\mathbb{K}^{n},|\cdot|\right)$ e $\left(\ell_{p},\left.|\cdot|\right|_{p}\right)$, em que $n \in \mathbb{N}$ e $p \in[1,+\infty]$, são exemplos de espaços de Banach.

$\mathrm{Na}$ seção anterior, definimos o conceito de tranformação linear entre $\mathbb{K}$-espaços vetoriais. Vamos, agora, analisar as transformações lineares entre espaços normados no que diz respeito à forma como estas se relacionam com as normas dos espaços em questão. Para tanto, comecemos com a seguinte definição:

Definição 2.6.7. Dados espaços normados $\left(X,\|\cdot\|_{X}\right)$ e $\left(E,\|\cdot\|_{E}\right)$ sobre $\mathbb{K}$, dizemos que uma transformação linear $T: X \longrightarrow E$ é limitada se existir $M>0$ tal que

$$
\|T(x)\|_{E} \leq M\|x\|_{X}
$$

para todo $x \in X$.

$\operatorname{Sejam~}\left(X,\|\cdot\|_{X}\right)$ e $\left(E,\|\cdot\|_{E}\right)$ espaços normados sobre $\mathbb{K}$ e $T: X \longrightarrow E$ uma transformação linear limitada. Nessas condições, é fácil ver que, para todo $M>0$ e todo $x \in X$ tal que $x \neq 0_{X}$,

$$
\|T(x)\|_{E} \leq M\|x\|_{X} \Leftrightarrow \frac{\|T(x)\|_{E}}{\|x\|_{X}} \leq M .
$$

Tendo em vista que a desigualdade à esquerda sempre vale para $x=0_{X}$, disso concluímos que existirá $M>0$ tal que

$$
\|T(x)\|_{E} \leq M\|x\|_{X} \quad \forall x \in X
$$

se, e somente se,

$$
\sup \left\{\frac{\|T(x)\|_{E}}{\|x\|_{X}}: x \in X \text { e } x \neq 0_{X}\right\}
$$

for finito. Isso nos sugere a seguinte definição:

DEFINIÇÃo 2.6.8. Dados espaços normados $\left(X,\|\cdot\|_{X}\right)$ e $\left(E,\|\cdot\|_{E}\right)$ sobre $\mathbb{K}$ e uma transformação linear limitada $T: X \longrightarrow E$, definimos a norma de $T$ como sendo o

$$
\sup \left\{\frac{\|T(x)\|_{E}}{\|x\|_{X}}: x \in X \text { e } x \neq 0_{X}\right\} .
$$

NotAÇÃo: A norma de uma transformação linear $T$ será denotada por $\|T\|$.

OBSERVAÇÃo 2.6.9. Sejam $\left(X,\|\cdot\|_{X}\right)$ e $\left(E,\|\cdot\|_{E}\right)$ espaços normados sobre $\mathbb{K}$ e $T: X \longrightarrow E$ uma transformação linear. Nessas condições, segue imediatamente de 2.6.8 que

$$
\|T(x)\|_{E} \leq\|T\|\|x\|_{X}
$$

para todo $x \in X$.

A próxima proposição mostra que, no caso das transformações lineares, as definições 2.3 .13 e 2.6.7 coincidem.

Proposição 2.6.10. Dados espaços normados $\left(X,\|\cdot\|_{X}\right)$ e $\left(E,\|\cdot\|_{E}\right)$ sobre $\mathbb{K}$ e uma transformação linear $T: X \longrightarrow E$, são equivalentes:

(1) $T$ é contínua em $0_{X}$;

(2) $T$ é contínua;

(3) $T$ é limitada. 


\subsection{TOPOLOGIA}

A seguir, vamos apresentar e discutir brevemente algumas noções básicas de Topologia, necessárias para o entendimento do restante do trabalho. O objetivo desta seção é servir como um guia aos leitores que, tendo estudado o assunto há muito tempo, já não se recordam tão bem dos cursos de Topologia. Apesar disso, ela também pode vir a ser útil àqueles que, embora não tenham, ainda, estudado Topologia Geral, possuam alguma familiaridade com a topologia dos espaços métricos.

\section{Conceitos BÁSICOS}

Comecemos apresentando as definições básicas.

Definição 2.7.1 (Topologia). Seja $X$ um conjunto não vazio. Uma topologia sobre $X$ é uma coleção $\tau$ de subconjuntos de $X$, chamados conjuntos abertos, tal que:

a) $\emptyset, X \in \tau$;

b) Se $U, V \in \tau$, então $U \cap V \in \tau$;

c) Se $\left(U_{i}\right)_{i \in I}$ é uma família de elementos de $\tau$, então $\bigcup_{i \in I} U_{i} \in \tau$.

DefiniÇão 2.7.2. Dadas topologias $\tau_{1}$ e $\tau_{2}$ sobre um mesmo conjunto $X$, dizemos que $\tau_{1}$ é mais fina que $\tau_{2}$ se $\tau_{2} \subseteq \tau_{1}$.

A noção de topologia motiva a seguinte definição:

DefiniçÃo 2.7.3 (ESPAÇO TOPOlÓGICO). Um espaço topológico é um par $(X, \tau)$, em que $X$ é um conjunto não vazio e $\tau$ é uma topologia sobre $X$.

Vejamos, a seguir, alguns exemplos de topologia, os quais mencionaremos ao longo do trabalho.

Exemplos 2.7.4. Seja $X$ um conjunto não vazio.

a) A coleção $\tau:=\{\emptyset, X\}$ é uma topologia sobre $X$, a qual denominamos topologia caótica.

b) A coleção $\tau:=\mathcal{P}(X)$ é uma topologia sobre $X$ conhecida como topologia discreta.

c) Sejam $\tau_{X}$ uma topologia sobre $X$ e $A \subseteq X$ um conjunto não vazio. Nessas condições, é um exercício simples mostrar que

$$
\tau_{A}:=\left\{U \cap A: U \in \tau_{X}\right\}
$$

é uma topologia sobre $A$. Dizemos que o par $\left(A, \tau_{A}\right)$ é um subespaço topológico de $\left(X, \tau_{X}\right)$.

d) A cada pseudométrica sobre $X$, podemos associar, univocamente, uma topologia. Com efeito, dada uma pseudométrica $d$ sobre $X$, é fácil ver que

$$
\tau_{d}:=\left\{U \subseteq X: \forall x \in U, \exists r>0 \text { t.q. } B_{X}^{d}(x, r) \subseteq U\right\}
$$

é uma topologia sobre $X$. De modo geral, dizemos que uma topologia $\tau$ sobre $X$ é pseudometrizável (resp., metrizável) se existir uma pseudométrica (resp., métrica) $d$ sobre $X$ tal que $\tau=\tau_{d}$. E, em caso afirmativo, uma tal pseudométrica (resp., métrica) é dita compatível. Observe que esse é o caso, por exemplo, das topologias caótica e discreta, as quais são induzidas, respectivamente, pela pseudométrica trivial e pela métrica discreta. 
OBSERVAÇÃo 2.7.5. Para simplificar a notação, denotaremos a topologia $\tau_{d_{\|\cdot\|}}$ induzida por uma norma $\|\cdot\|$ sobre um conjunto $X$ simplesmente por $\tau_{\|\cdot\| \cdot}$.

Recordemos, ainda, as seguintes definições:

Definição 2.7.6 (Conjunto FeChado). Dado um espaço topológico $(X, \tau)$, dizemos que um subconjunto $F \subseteq X$ é fechado em $(X, \tau)$ se $X \backslash F \in \tau$.

Definição 2.7.7. Sejam $(X, \tau)$ um espaço topológico, $A \subseteq X$ um subconjunto qualquer e $x \in X$ um ponto dado. Nessas condições:

a) Dizemos que $x$ é um ponto interior a $A$ se existir $U \in \tau$ tal que $x \in U$ e $U \subseteq A$;

b) Dizemos que $x$ é um ponto aderente a $A$ se, para todo $U \in \tau$ tal que $x \in U$, valer que $U \cap A \neq \emptyset$.

O conjunto dos pontos interiores a $A$ em $(X, \tau)$ é denominado interior de $A$ e será denotado por $\operatorname{int}_{\tau}(A)$. Já o conjunto dos pontos aderentes a $A$ em $(X, \tau)$ recebe o nome de fecho de $A$, e será indicado por $\bar{A}^{\tau}$. Quando a topologia $\tau$ estiver clara pelo contexto, escreveremos apenas $\operatorname{int}(A)$ e $\bar{A}$ para nos referirmos, respectivamente, ao interior e ao fecho de $A$.

OBSERVAÇÃo 2.7.8. O interior de um conjunto $A \subseteq X$ em um espaço topológico $(X, \tau)$ é sempre aberto em $(X, \tau)$ e, analogamente, o fecho de $A$ é sempre fechado.

Definição 2.7.9 (Subconjunto Denso). Dada uma topologia $\tau$ sobre um conjunto $X$, dizemos que um subconjunto $A \subseteq X$ é denso em $(X, \tau)$ se $\bar{A}^{\tau}=X$.

Definição 2.7.10. Dizemos que um espaço topológico $(X, \tau)$ é separável se existir um subconjunto enumerável $A \subseteq X$ denso em $(X, \tau)$.

Definição 2.7.11 (VizinhançA). Sejam $(X, \tau)$ um espaço topológico e $x \in X$ um ponto dado. Dizemos que um subconjunto $V \subseteq X$ é uma vizinhança de $x$ em $(X, \tau)$ se existir $U \in \tau$ tal que $x \in U$ e $U \subseteq V$.

Uma subclasse bastante importante de espaços topológicos é a dos espaços de Hausdorff. Intuitivamente, um espaço de Hausdorff é um espaço topológico no qual é possível separar pontos distintos por abertos disjuntos. No que segue, procuraremos tornar essa ideia um pouco mais precisa.

DefiniçÃo 2.7.12 (EspaÇO De Hausdorff). Dizemos que um espaço topológico $(X, \tau)$ é um espaço de Hausdorff se, para todos $x, y \in X$ tais que $x \neq y$, existirem $U, V \in \tau$ tais que $x \in U, y \in V$ e $U \cap V=\emptyset$.

Exemplos 2.7.13. Seja $X$ um conjunto não vazio.

a) O espaço topológico $\left(X, \tau_{d}\right)$, em que $d$ é uma métrica sobre $X$ e $\tau_{d}$ é a topologia induzida por $d$ (veja o exemplo 2.7.4-c)) é um espaço de Hausdorff. Com efeito, sendo $d$ uma métrica sobre $X$, temos que $d(x, y)>0$ para todos $x, y \in X$ tais que $x \neq y$. E, sendo assim, se $x, y \in X$ forem tais que $x \neq y$, basta tomarmos $\varepsilon=\frac{d(x, y)}{2}$ para que tenhamos $B_{X}^{d}(x, \varepsilon), B_{X}^{d}(y, \varepsilon) \in \tau$, $x \in B_{X}^{d}(x, \varepsilon), y \in B_{X}^{d}(y, \varepsilon) \mathrm{e}$

$$
B_{X}^{d}(x, \varepsilon) \cap B_{X}^{d}(y, \varepsilon)=\emptyset .
$$

b) O espaço $(X, \tau)$, em que $\tau$ é a topologia caótica, não é de Hausdorff (uma vez que, nesse caso, o único aberto não vazio é o próprio $X)$. 
c) A propriedade "ser ou não de Hausdorff" é herdada por subespaços topológicos. Isto é, se $\tau$ for uma topologia sobre $X$ tal que $(X, \tau)$ é um espaço de Hausdorff, então, para todo $A \subseteq X$ não vazio, $\left(A, \tau_{A}\right)$ será, também, um espaço de Hausdorff.

Embora periférica, a noção de compacidade também está presente no trabalho. Por essa razão, definiremos, a seguir, o conceito de conjunto compacto em um espaço topológico.

Definição 2.7.14 (Conjunto COMpacto). Dado um espaço topológico $(X, \tau)$, dizemos que um subconjunto $A \subseteq X$ é compacto em $(X, \tau)$ se, para toda família $\left(U_{i}\right)_{i \in I}$ de subconjuntos abertos tal que

$$
A \subseteq \bigcup_{i \in I} U_{i},
$$

existir um subconjunto finito $F \subseteq I$ tal que

$$
A \subseteq \bigcup_{i \in F} U_{i} .
$$

Se $\bar{A}^{\tau}$ for compacto em $(X, \tau)$, dizemos que $A$ é relativamente compacto.

A próxima proposição é uma consequência simples da definição 2.7.14.

Proposição 2.7.15. Em um espaço topológico, todo conjunto fechado contido em um conjunto compacto é, também, um conjunto compacto.

\section{BASES E BASES LOCAIS}

Muitas vezes, é conveniente descrever a topologia de um espaço por meio da coleção de vizinhanças de cada um de seus pontos. Isso motiva o importante conceito de base local, o qual enunciaremos a seguir.

DefiniçÃo 2.7.16 (BASE LOCAL). Sejam $(X, \tau)$ um espaço topológico e $x \in X$ um ponto dado. Dizemos que uma coleção $\mathcal{V} \subseteq \mathcal{P}(X)$ é uma base local de $x$ em $(X, \tau)$ se cada elemento de $\mathcal{V}$ for uma vizinhança de $x$ e, para cada $U \in \tau$ tal que $x \in U$, existir $V \in \mathcal{V}$ tal que $V \subseteq U$.

OBSERVAÇÃo 2.7.17. Seja $(X, \tau)$ um espaço topológico, e, para cada $x \in X$, seja $\mathcal{V}_{x}$ uma base local de $x$ em $(X, \tau)$. Nessas condições, segue diretamente das definições 2.7.11 e 2.7.16, e da propriedade c) da definição 2.7.1, que

$$
\tau=\left\{U \subseteq X: \forall x \in U, \exists V \in \mathcal{V}_{x} \text { t.q. } V \subseteq U\right\}
$$

Exemplos 2.7.18. Sejam $X$ um conjunto não vazio e $x \in X$ um ponto dado.

a) A coleção

$$
\mathcal{V}_{x}:=\{U \in \tau: x \in U\}
$$

é uma base local de $x$ em $(X, \tau)$ qualquer que seja a topologia $\tau$ sobre $X$.

b) A coleção $\mathcal{V}_{x}:=\{\{x\}\}$ é uma base local de $x$ em relação à topologia discreta.

c) Seja $A \subseteq X$ um subconjunto ao qual $x$ pertence. Nessas condições, se $\mathcal{V}_{x}$ for uma base local de $x$ em $(X, \tau)$, então

$$
\mathcal{V}_{x}^{A}:=\left\{V \cap A: V \in \mathcal{V}_{x}\right\}
$$

será uma base local de $x$ em $\left(A, \tau_{A}\right)$. 
d) Dada uma pseudométrica $d$ sobre $X$, é imediato ver que

$$
\mathcal{V}_{x}:=\left\{B\left(x, \frac{1}{n}\right): n \in \mathbb{N}\right\}
$$

é uma base local (enumerável) de $x$ em $\left(X, \tau_{d}\right)$.

A proposição a seguir sintetiza algumas propriedades básicas das bases locais.

Proposição 2.7.19. Seja $(X, \tau)$ um espaço topológico e, para cada $x \in X$, seja $\mathcal{V}_{x}$ uma base local de $x$ em $(X, \tau)$. Nessas condições:

a) $x \in V$ para todo $V \in \mathcal{V}_{x}$;

b) Para todos $V_{1}, V_{2} \in \mathcal{V}_{x}$, existe $W \in \mathcal{V}_{x}$ tal que $W \subseteq V_{1} \cap V_{2}$;

c) Para todo $V \in \mathcal{V}_{x}$, existe $V_{0} \in \mathcal{V}_{x}$ tal que, para todo $y \in \mathcal{V}_{0}$, existe $W \in \mathcal{V}_{y}$ tal que $W \subseteq V$.

O próximo resultado é uma espécie de recíproca da proposição 2.7.19.

Proposição 2.7.20. Seja $X$ um conjunto arbitrário e, para cada $x \in X$, seja $\mathcal{V}_{x}$ uma coleção de subconjuntos de $X$ satisfazendo as propriedades a), b) e c) da proposição 2.7.19. Nessas condições, a coleção

$$
\tau:=\left\{U \subseteq X: \forall x \in U, \exists V \in \mathcal{V}_{x} \text { t.q. } V \subseteq U\right\}
$$

é uma topologia sobre $X$ e, para cada $x \in X, \mathcal{V}_{x}$ é uma base local de $x$ em $(X, \tau)$.

Da mesma forma como podemos descrever a coleção de vizinhanças de um ponto $x \in X$ em um espaço topológico $(X, \tau)$ especificando uma base local de vizinhanças de $x$ em $(X, \tau)$, é possível, também, caracterizar a topologia como um todo a partir de certas subcoleções de conjuntos abertos. Coleções com tal propriedade são chamadas bases de $(X, \tau)$. Formalmente, temos a seguinte definição:

Definição 2.7.21 (BASE). Seja $(X, \tau)$ um espaço topológico. Dizemos que uma subcoleção $\mathscr{B} \subseteq \tau$ é uma base de $(X, \tau)$ (ou, simplesmente, uma base de $\tau$ ) se, para todo $U \in \tau$ e todo $x \in U$, existir $B \in \mathscr{B}$ tal que $x \in B$ e $B \subseteq U$.

OBSERVAÇÃO 2.7.22.

a) Todo espaço topológico $(X, \tau)$ tem base. Com efeito, a coleção $\tau$ é, ela própria, uma base de $(X, \tau)$

b) Uma subcoleção $\mathscr{B} \subseteq \mathcal{P}(X)$ é base de um espaço topológico $(X, \tau)$ se, e somente se,

$$
\tau=\{U \subseteq X: \forall x \in U, \exists B \in \mathscr{B} \text { t.q. } x \in B \text { e } B \subseteq U\}
$$

Vejamos, a seguir, alguns exemplos.

Exemplos 2.7.23. Seja $X$ um conjunto não vazio.

a) A coleção $\mathscr{B}:=\{\{x\}: x \in X\}$ é uma base de $X$ em relação à topologia discreta. 
b) Sejam $A \subseteq X$ um conjunto não vazio e $\tau$ uma topologia sobre $X$. Nessas condições, não é difícil mostrar que, se $\mathscr{B}$ for uma base de $(X, \tau)$, então

$$
\mathscr{B}_{A}:=\{B \cap A: B \in \mathscr{B}\}
$$

será uma base de $\left(X, \tau_{A}\right)$ (veja o item c) do exemplo 2.7.4).

c) Dada uma pseudométrica $d$ sobre $X$, segue da definição de $\tau_{d}$ que a coleção

$$
\mathscr{B}_{d}:=\left\{B_{X}^{d}(x, r): x \in X \text { e } r>0\right\}
$$

é uma base de $\left(X, \tau_{d}\right)$. Observe que o item a) é um caso particular deste exemplo, uma vez que a métrica discreta induz sobre $X$ a topologia discreta.

A item a) da observação 2.7.22 nos mostra que todo espaço topológico possui, ao menos, uma base. Isso nos leva a considerar o problema inverso:

Como saber se uma dada coleção $\mathbb{B} \subseteq \mathcal{P}(X)$ é base de alguma topologia sobre $X$ ?

A partir do item b) da observação 2.7.22, é fácil ver que isso ocorre se, e somente se, a coleção

$$
\tau:=\{U \subseteq X: \forall x \in U, \exists B \in \mathscr{B} \text { t.q. } x \in B \text { e } B \subseteq U\}
$$

for uma topologia sobre $X$. E, tendo isso em mente, não é difícil provar o seguinte critério:

Proposição 2.7.24. Seja $X$ um conjunto não vazio. Nessas condições, uma coleção $\mathscr{B} \subseteq$ $\mathcal{P}(X)$ é base de uma topologia sobre $X$ se, e somente se:

a) Para todo $x \in X$, existe $B \in \mathscr{B}$ tal que $x \in B$;

b) Dados $B_{1}, B_{2} \in \mathscr{B}$ e $x \in B_{1} \cap B_{2}$, existe $B \in \mathscr{B}$ tal que $x \in B$ e $B \subseteq B_{1} \cap B_{2}$.

A seguir, vamos relacionar os conceitos de base e base local. Mais especificamente, vamos mostrar como obter uma base local a partir de uma base e, reciprocamente, obter uma base a partir de uma coleção de bases locais.

TeORema 2.7.25. Sejam $(X, \tau)$ um espaço topológico, $\mathscr{B} \subseteq \tau$ uma base de $(X, \tau)$ e, para cada $x \in X$, seja $\mathcal{V}_{x} \subseteq \mathcal{P}(X)$ uma base local de $x$ em $(X, \tau)$. Nessas condições:

a) $A$ coleção

$$
\mathscr{C}:=\bigcup_{x \in X}\left(\mathcal{V}_{x} \cap \tau\right)
$$

é base de $(X, \tau)$;

b) Para cada $x \in X$, a coleção

$$
\mathscr{B}(x):=\{B \in \mathscr{B}: x \in B\}
$$

é uma base local de $x$ em $(X, \tau)$.

\section{TOPOLOGIA PRODUTO}

Nesta seção, vamos mostrar como, dados espaços topológicos $\left(X_{1}, \tau_{1}\right), \ldots,\left(X_{n}, \tau_{n}\right)$, em que $n \in \mathbb{N}$, podemos obter uma topologia sobre

$$
X:=\prod_{i=1}^{n} X_{i}:=\left\{\left(x_{1}, \ldots, x_{n}\right): x_{i} \in X_{i} \forall i \in\{1, \ldots, n\}\right\}
$$


a partir de $\tau_{1}, \ldots, \tau_{n}$.

Para isso, consideremos a coleção

$$
\mathscr{B}_{\text {prod }}:=\left\{\prod_{i=1}^{n} U_{i}: U_{i} \in \tau_{i} \forall i \in\{1, \ldots, n\}\right\},
$$

em que, dados $U_{1} \in \tau_{1}, \ldots, U_{n} \in \tau_{n}$,

$$
\prod_{i=1}^{n} U_{i}:=\left\{\left(x_{1}, \ldots, x_{n}\right) \in X: x_{i} \in U_{i} \forall i \in\{1, \ldots, n\}\right\} .
$$

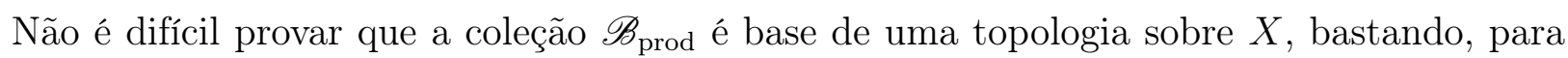

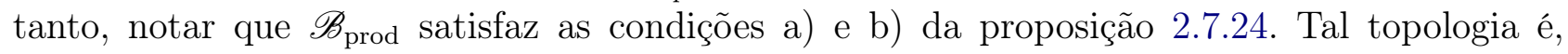
comumente, chamada de topologia produto associada a $\left(\tau_{1}, \ldots, \tau_{n}\right)$, e será denotada por $\tau_{\text {prod }}$.

OBSERVAÇÃo 2.7.26. Observe que, ao definir a topologia produto, limitamo-nos a considerar um número finito de espaços topológicos. Apesar disso, é possível estender essa definição a uma família arbitrária (enumerável ou não) de espaços topológicos. No entanto, para os fins deste trabalho, não será necessária tamanha generalidade.

\section{Pseudométricas equivalentes}

Dada uma pseudométrica $d$ sobre um conjunto $X$, é imediato ver que $2 d$ é também uma pseudométrica sobre $X$ e que $d$ e $2 d$ induzem sobre $X$ a mesma topologia. Essa observação, embora trivial, nos mostra que duas pseudométricas sobre um mesmo conjunto podem dar origem à mesma topologia, sendo, portanto, topologicamente equivalentes. Com efeito, é fácil ver que a relação $\sim$, definida sobre a classe de todas as pseudométricas de um dado conjunto $X$ de modo que $d_{1} \sim d_{2}$ se, e somente se, $\tau_{d_{1}}=\tau_{d_{2}}$, é uma relação de equivalência. Isso nos sugere a seguinte definição:

Definição 2.7.27 (Pseudométricas EQUivalentes). Duas pseudométricas sobre um mesmo conjunto $X$ são ditas equivalentes se induzirem sobre $X$ a mesma topologia.

A próxima proposição, cuja demonstração é direta, nos fornece uma condição suficiente para que duas pseudométricas sobre um mesmo conjunto $X$ sejam equivalentes.

Proposição 2.7.28. Sejam $d_{1}$ e $d_{2}$ duas pseudométricas sobre um mesmo conjunto $X$. Nessas condições, se existirem constantes $A, B>0$ tais que

$$
A d_{1}(x, y) \leq d_{2}(x, y) \leq B d_{1}(x, y) \quad \forall x, y \in X,
$$

então $\tau_{d_{1}}=\tau_{d_{2}}$.

A recíproca da proposição 2.7.28 não vale em geral. Com efeito, não é difícil mostrar que as métricas $d_{1}$ e $d_{2}$ tais que

$$
d_{1}(x, y)=|x-y|
$$

$\mathrm{e}$

$$
d_{2}(x, y)=\left|\frac{1}{x}-\frac{1}{y}\right|
$$

induzem a mesma topologia sobre $X:=] 0,+\infty[$, mas não existem constantes $A, B>0$ tais que

$$
A d_{1}(x, y) \leq d_{2}(x, y) \leq B d_{1}(x, y) \quad \forall x, y \in X .
$$


No capítulo 3, mostraremos que é possível garantir que duas pseudométricas sobre um mesmo conjunto sejam equivalentes a partir de condições um pouco menos restritivas. Em outras palavras, vamos "afrouxar" um pouco as hipóteses da proposição 2.7.28.

Embora, em geral, o critério dado pela proposição 2.7.28 não sirva para demonstrar que duas pseudométricas não são equivalentes, nosso próximo resultado mostra que a situação é diferente no caso em que ambas as pseudométricas são induzidas por normas.

TeOrema 2.7.29. Duas normas, $\|\cdot\|_{1} e\|\cdot\|_{2}$, sobre um mesmo $\mathbb{K}$ - espaço vetorial $X$ induzem a mesma topologia sobre $X$ se, e somente se, existem constantes $A, B>0$ tais que

$$
A\|x\|_{1} \leq\|x\|_{2} \leq B\|x\|_{1}
$$

para todo $x \in X$.

O teorema 2.7.29 motiva a seguinte definição:

Definição 2.7.30 (Normas EQuivalentes). Duas normas, $\|\cdot\|_{1}$ e $\|\cdot\|_{2}$, sobre um mesmo $\mathbb{K}$ - espaço vetorial $X$ são ditas equivalentes se existirem constantes $A, B>0$ tais que

$$
A\|x\|_{1} \leq\|x\|_{2} \leq B\|x\|_{1}
$$

para todo $x \in X$.

Exemplo 2.7.31. As normas $\|\cdot\|_{1},\|\cdot\|_{2}$ e $\|\cdot\|_{\max }$, definidas no exemplo 2.6.3-a), são todas equivalentes entre si, pois, para todo $\left(x_{1}, \ldots, x_{n}\right) \in \mathbb{K}^{n}$,

$$
\max \left\{\left|x_{1}\right|, \ldots,\left|x_{n}\right|\right\} \leq \sum_{i=1}^{n}\left|x_{i}\right| \leq n \max \left\{\left|x_{1}\right|, \ldots,\left|x_{n}\right|\right\},
$$

e

$$
\max \left\{\left|x_{1}\right|, \ldots,\left|x_{n}\right|\right\} \leq\left(\sum_{i=1}^{n}\left|x_{i}\right|^{2}\right)^{\frac{1}{2}} \leq \sqrt{n} \max \left\{\left|x_{1}\right|, \ldots,\left|x_{n}\right|\right\} .
$$

Voltaremos à definição 2.7.30 no capítulo 5 , ao estudarmos o espaço $\left(\ell_{p}(E,\|\cdot\|),\|\cdot\|_{p}\right){ }^{1}$

\section{CONTINUIDADE}

Uma das principais motivações para se estudar espaços topológicos reside no fato de que eles permitem generalizar noções como a continuidade a partir do ambiente substancialmente mais restrito dos espaços métricos. Vejamos, a seguir, como fica a definição de continuidade no contexto mais geral das funções entre espaços topológicos.

Definição 2.7.32 (Continuidade). Sejam $\left(X, \tau_{X}\right)$ e $\left(Y, \tau_{Y}\right)$ espaços topológicos e $x \in X$ um ponto dado. Dizemos que uma função $f: X \longrightarrow Y$ é contínua em $x$ se, para todo $U \in \tau_{Y}$ tal que $f(x) \in U$, existir $V \in \tau_{X}$ tal que $x \in V$ e $f(V) \subseteq U$.

Como sempre, se $f: X \longrightarrow Y$ for contínua em $x$ para todo $x \in X$, diremos, apenas, que $f$ é contínua. E, se, além disso, $f$ for bijetora e $f^{-1}: Y \longrightarrow X$ for contínua, diremos que $f$ é um homeomorfismo.

ExEMPLO 2.7.33. A definição 2.7.32 nos permite recuperar a definição usual de continuidade (em termos de épsilons e deltas) no caso das funções entre espaços pseudométricos. Assim sendo,

\footnotetext{
${ }^{1}$ Em que $(E,\|\cdot\|)$ é um espaço normado e $p \in[1,+\infty[$.
} 
se as topologias $\tau_{X}$ e $\tau_{Y}$ forem induzidas por pseudométricas $d$ e $\partial$ sobre $X$ e $Y$, respectivamente, uma função $f: X \longrightarrow Y$ será contínua em um ponto $x \in X$ se, e somente se, para todo $\varepsilon>0$, existir $\delta>0$ tal que, para todos $x, y \in X$,

$$
d(x, y)<\delta \Rightarrow \partial(f(x), f(y))<\varepsilon .
$$

Como a topologia caótica e a topologia discreta são induzidas, respectivamente, pela métrica discreta e pela pseudométrica trivial, disso resulta, em particular, que, se $\tau_{Y}$ for a topologia caótica ou $\tau_{X}$ for a topologia discreta, toda função $f: X \longrightarrow Y$ será contínua (veja o exemplo 2.3.14).

Observe, no entanto, que não é possível utilizar uma definição análoga a 2.7 .32 para estender o conceito de continuidade uniforme a funções entre espaços topológicos.

\section{FiLtros E IDEAIS}

Por fim, vamos encerrar a nossa revisão de Topologia discutindo, rapidamente, as definições de filtro e de ideal.

Definição 2.7.34 (Filtro). Seja $X$ um conjunto não vazio. Um filtro sobre $X$ é uma coleção não vazia $\mathscr{F} \subseteq \mathcal{P}(X)$ com as seguintes propriedades:

a) $\emptyset \notin \mathscr{F}$;

b) Se $F, G \in \mathscr{F}$, então $F \cap G \in \mathscr{F}$;

c) Se $F \in \mathscr{F}$ e $F \subseteq G \subseteq X$, então $G \in \mathscr{F}$.

Vejamos, a seguir, alguns exemplos de filtros.

Exemplos 2.7.35. Sejam $X$ um conjunto não vazio e $x \in X$ um ponto dado.

a) A coleção

$$
\mathscr{F}:=\{F \subseteq X: x \in F\}
$$

é um filtro sobre $X$, o qual denominamos filtro principal gerado por $x$.

b) A coleção das vizinhanças de $x$ em um espaço topológico $(X, \tau)$ é um filtro sobre $X$. Isso é uma consequência direta da proposição 2.7.19.

Dual à noção de filtro é o conceito de ideal. A ideia por trás dessa dualidade é a de que, enquanto os elementos de um filtro sobre $X$ correspondem, em um certo sentido, aos subconjuntos "grandes" de $X$, os elementos de um ideal de $X$ seriam os subconjuntos "pequenos", de "tamanho" negligenciável. Mais precisamente, temos a seguinte definição:

DEFINIÇÃo 2.7.36 (IDEAL). Seja $X$ um conjunto não vazio. Um ideal sobre $X$ é uma coleção não vazia $\mathcal{A} \subseteq \mathcal{P}(X)$ tal que, para todos $A, B \subseteq X$ :

a) Se $A, B \in \mathcal{A}$, então $A \cup B \in \mathcal{A}$;

b) Se $A \in \mathcal{A}$ e $B \subseteq A$, então $B \in \mathcal{A}$.

Exemplos 2.7.37. Seja $X$ um conjunto não vazio.

a) Dado um subconjunto qualquer $A \subseteq X$, a coleção $\mathcal{A}:=\mathcal{P}(A)$ é um ideal sobre $X$. E, se $X$ for finito, todos os ideais de $X$ são dessa forma.

b) A coleção dos subconjuntos finitos de $X$ é um ideal sobre $X$. 


\subsection{GRUPOS TOPOLÓGICOS}

Nesta última seção, vamos introduzir, brevemente, o conceito de grupo topológico. Para tanto, recordemos, primeiramente, as definições de grupo, subgrupo e conjunto gerador.

Definição 2.8.1 (Grupo). Um grupo é um par $(G, \cdot)$, em que $G$ é um conjunto não vazio e $\cdot: G \times G \longrightarrow G$ é uma operação binária sobre $G$ tal que

a) $(a \cdot b) \cdot c=a \cdot(b \cdot c)$ para todos $a, b, c \in G$;

b) Existe um único $\tilde{e} \in G$, o qual denotaremos por $e$, tal que $a \cdot \tilde{e}=a$ para todo $a \in G$;

c) Para todo $a \in G$, existe $b \in G$ tal que $a \cdot b=b \cdot a=e$.

Se, em adição às propriedades a), b) e c), valer também que $a \cdot b=b \cdot a$ quaisquer que sejam $a, b \in G$, dizemos que $(G, \cdot)$ é um grupo abeliano.

DeFiniçÃo 2.8.2 (CONJunto Gerador). Dado um grupo $(G, \cdot)$, dizemos que um subconjunto $A \subseteq G$ é um conjunto gerador de $(G, \cdot)$ (ou que $A$ gera $(G, \cdot)$ ) se, para cada $g \in G$, existirem $n \in \mathbb{N}$ e $a_{1}, \ldots, a_{n} \in G$ tais que

$$
g=a_{1} \cdot \ldots \cdot a_{n}
$$

No caso particular em que $(G, \cdot)$ possui um conjunto gerador finito, dizemos que $(G, \cdot)$ é finitamente gerado.

OBSERVAÇÃo 2.8.3. Todo grupo $(G, \cdot)$ possui, ao menos, um conjunto gerador, uma vez que $G$ é, ele próprio, um gerador de $(G, \cdot)$.

Definição 2.8.4 (Subgrupo). Sejam $(G, \cdot)$ um grupo, $H \subseteq G$ um subconjunto não vazio e $\star: H \times H \longrightarrow H$ uma operação sobre $H$. Nessas condições. dizemos que $(H, \star)$ é um subgrupo de $(G, \cdot)$ se $(H, \star)$ for um grupo e $g \star h=g \cdot h$ para todos $g, h \in H$.

Uma noção naturalmente associada ao conceito de grupo é a de conjunto invariante à esquerda (ou à direita). Formalmente, temos a seguinte definição:

DefiniçÃo 2.8.5 (COnjuntos invariantes À ESQuerda). Dado um grupo $(G, \cdot)$, dizemos que um subconjunto $A \subseteq G$ é invariante à esquerda em $(G, \cdot)$ se $\lambda x \in A$ quaisquer que sejam $\lambda \in G$ e $x \in A$. Analogamente, dizemos que um subconjunto $E \subseteq G \times G$ é invariante à esquerda em $(G, \cdot)$ se, para todo $(x, y) \in G \times G$ e todo $\lambda \in G$,

$$
(x, y) \in E \Rightarrow(\lambda x, \lambda y) \in E .
$$

A partir da definição 2.8.5, pode-se provar, facilmente, a seguinte proposição:

ProposiÇão 2.8.6. Seja $(G, \cdot)$ um grupo. Nessas condições:

a) $\Delta(G)$ é invariante à esquerda;

b) Se $E \subseteq G \times G$ é invariante à esquerda, então $E^{-1}$ é invariante à esquerda;

c) Se $E, F \subseteq G \times G$ são invariantes à esquerda, então $E \circ F$ é invariante à esquerda;

d) $S e\left(E_{i}\right)_{i \in I}$ é uma família não vazia de subconjuntos de $G \times G$ invariantes à esquerda, então $\bigcup_{i \in I} E_{i} e \bigcap_{i \in I} E_{i}$ são invariantes à esquerda. 
Uma vez estabelecidos os conceitos mais básicos, passemos, sem mais demora, à definição de grupo topológico.

DEFiniçÃo 2.8.7 (GRUPo TOPOlÓGICO). Um grupo topológico é uma tripla $(G, \cdot, \tau)$, em que $(G, \cdot)$ é um grupo e $\tau$ é uma topologia sobre $G$ que torna as operações

$$
\begin{aligned}
m: G \times G & \longrightarrow G \\
(x, y) & \longmapsto x y
\end{aligned}
$$

e

$$
\begin{aligned}
i: G & \longrightarrow G \\
x & \longmapsto x^{-1}
\end{aligned}
$$

contínuas.

ExEMPLOS 2.8.8.

a) Se $(E,\|\cdot\|)$ é um espaço normado e $\tau_{\|\cdot\|}$ é a topologia de $E$ induzida pela norma, então $\left(E,+, \tau_{\|\cdot\|}\right)$ é um grupo topológico. Disso resulta, em particular, que $\left(\mathbb{R}^{n},+, \tau_{\|\cdot\|}\right)$, em que $n \in \mathbb{N}$, é um grupo topológico.

Demonstração. Para provarmos que $\left(X,+, \tau_{\|\cdot\|}\right)$ é um grupo topológico, é suficiente mostrarmos que as funções

$$
\begin{aligned}
s: X \times X & \longrightarrow X \\
(x, y) & \longmapsto x+y
\end{aligned}
$$

e

$$
\begin{aligned}
i: X & \longrightarrow X \\
x & \longmapsto-x
\end{aligned}
$$

são contínuas.

A continuidade de $i$ é imediada, uma vez que, para todos $x, x_{0} \in X$, temos que

$$
\left\|i(x)-i\left(x_{0}\right)\right\|=\left\|(-x)-\left(-x_{0}\right)\right\|=\left\|x-x_{0}\right\| .
$$

Mostremos, agora, a continuidade de $s$. Para isso, fixemos $\left(x_{0}, y_{0}\right) \in X \times X$ de modo arbitrário, e vamos provar que $s$ é contínua em $\left(x_{0}, y_{0}\right)$.

Seja $\varepsilon>0$ dado. Inicialmente, observe que $B_{X}\left(x_{0}, \frac{\varepsilon}{2}\right) \times B_{X}\left(y_{0}, \frac{\varepsilon}{2}\right) \in\left(\tau_{\|\cdot\|}\right)_{\text {prod }}$, uma vez que $B_{X}\left(x_{0}, \frac{\varepsilon}{2}\right), B_{X}\left(y_{0}, \frac{\varepsilon}{2}\right) \in \tau_{\|\cdot\|}$. Além disso, também é fácil ver que, para todo $(x, y) \in X \times X$,

$$
\begin{aligned}
(x, y) \in B_{X}\left(x_{0}, \frac{\varepsilon}{2}\right) \times B_{X}\left(y_{0}, \frac{\varepsilon}{2}\right) & \Rightarrow\left\|s(x, y)-s\left(x_{0}, y_{0}\right)\right\|=\left\|(x+y)-\left(x_{0}+y_{0}\right)\right\| \\
& =\left\|\left(x-x_{0}\right)+\left(y-y_{0}\right)\right\| \leq\left\|x-x_{0}\right\|+\left\|y-y_{0}\right\| \\
& <\frac{\varepsilon}{2}+\frac{\varepsilon}{2}=\varepsilon,
\end{aligned}
$$

do que segue que

$$
s\left(B_{X}\left(x_{0}, \frac{\varepsilon}{2}\right) \times B_{X}\left(y_{0}, \frac{\varepsilon}{2}\right)\right) \subseteq B_{X}\left(s\left(x_{0}, y_{0}\right), \varepsilon\right) .
$$


E, como $\varepsilon>0$ é arbitrário, disso concluímos que $s$ é contínua em $\left(x_{0}, y_{0}\right)$.

b) Sejam $(G, \cdot)$ um grupo qualquer e $\tau_{d}$ a topologia discreta sobre $G$. Nessas condições, é fácil ver que a topologia produto induzida por $\tau_{d}$ sobre $G \times G$ é também a topologia discreta e, portanto, quaisquer funções de $G$ em $G$ e de $G \times G$ em $G$ são contínuas, a partir do que segue que $\left(G, \cdot, \tau_{d}\right)$ é um grupo topológico.

c) Se $(G, \cdot, \tau)$ for um grupo topológico e $(H, \cdot)$ for um subgrupo de $(G, \cdot)$, então $\left(H, \cdot, \tau_{H}\right)$, em que $\tau_{H}$ é a topologia de subespaço induzida por $\tau$ sobre $H$, será, também, um grupo topológico.

A proposição a seguir, com a qual encerramos o capítulo, é uma consequência direta da definição 2.8.7.

Proposição 2.8.9. Seja $(G, \cdot, \tau)$ um grupo topológico. Nessas condições:

a) $i: G \longrightarrow G$ tal que $i(x)=x^{-1}$ é um homeomorfismo, e $i^{-1}=i$;

b) Para cada $g \in G, f_{g}: G \longrightarrow G$ tal que $f_{g}(h)=g \cdot h$ é um homeomorfismo, e $f_{g}^{-1}=f_{g^{-1}}$. 


\section{Capítulo 3}

\section{ESPAÇOS UNIFORMES}

Noções como continuidade uniforme ou convergência uniforme, as quais podem ser facilmente definidas para funções e sequências de funções entre espaços métricos, perdem-se quando são levadas em conta apenas as propriedades topológicas dos espaços em questão. Isso porque, em um espaço topológico, as vizinhanças de um ponto (e, portanto, também a noção de "distância topológica") usualmente dependem da "localização" do ponto no espaço. A diferença crucial da métrica em relação à topologia, nesse caso, consiste no fato de que distância é uma noção que pode ser aplicada uniformemente a pares de pontos do espaço independentemente de suas localizações.

Apesar disso, uma métrica carrega mais informação do que o necessário para introduzir noções como a continuidade uniforme. Para ilustrar esse ponto, basta notar que uma função qualquer $f: \mathbb{R} \longrightarrow \mathbb{R}$ será uniformemente contínua em relação à métrica usual se, e somente se, for uniformemente contínua em relação a $d^{\prime}$, em que, para todos $x, y \in \mathbb{R}$, $d^{\prime}(x, y)=\min \{1,|x-y|\}$. Assim sendo, toda a informação sobre a geometria de larga escala do espaço acaba se mostrando irrelevante quando o que está em jogo são propriedades locais como a continuidade uniforme. E, por essa razão, seria desejável definir uma estrutura sobre um conjunto que correspondesse a um meio termo entre a métrica e a topologia no que diz respeito à continuidade uniforme. Uma tal estrutura é comumente chamada de estrutura uniforme. E, por sua vez, um espaço uniforme nada mais é do que um par $(X, \mathscr{D})$, em que $X$ é um conjunto não vazio e $\mathscr{D}$ é uma estrutura uniforme sobre $X$.

A abordagem das estruturas uniformes com que trabalharemos aqui foi primeiramente introduzida no livro Topologie Générale, de Nicolas Bourbaki [Bou66], embora o conceito já estivesse presente na literatura pelo menos desde 1937, ano de publicação do livro Sur les Espaces à Structure Uniforme et sur la Topologie Générale por André Weil [Wei37]. De um modo geral, uma estrutura uniforme está para a continuidade uniforme assim como uma topologia está para a continuidade. Nesse sentido, espaços topológicos e espaços uniformes correspondem aos ambientes naturais para a generalização das noções de continuidade e continuidade uniforme, respectivamente, a partir do ambiente consideravelmente mais restrito dos espaços métricos.

Neste capítulo, apresentaremos uma introdução elementar à teoria dos espaços uniformes. Para mais detalhes sobre o assunto, recomendamos a leitura do livro General Topology, de Stephen Willard [Wil70], no qual se baseia uma parte considerável do material do capítulo. Na primeira seção, atribuiremos significados precisos a noções como "estrutura uniforme", "espaço uniforme", "base de uma uniformidade", entre outras, as quais serão, em seguida, trabalhadas por meio de exemplos, dentre os quais destacam-se a estrutura uniforme induzida por uma pseudométrica e a estrutura uniforme à esquerda de um grupo topológico. Posteriormente, exploraremos, em mais detalhes, a conexão entre estrutura uniforme e topologia. E, por fim, vamos estudar certos tipos de funções entre espaços uniformes. Em particular, mostraremos 
como generalizar, de forma natural, conceitos como continuidade uniforme, mergulho uniforme e isomorfismo uniforme para o cenário consideravelmente mais abrangente das funções entre espaços uniformes.

\subsection{ESTRUTURAS UNIFORMES}

Daremos início a esta seção definindo, de forma precisa, o importante conceito de estrutura uniforme sobre um conjunto $X$.

DeFiniçÃo 3.1.1 (Estrutura Uniforme). Seja $X$ um conjunto não vazio. Uma estrutura uniforme (ou uniformidade) sobre $X$ é uma coleção não vazia $\mathscr{D}$ de subconjuntos de $X \times X$, chamados vizinhanças diagonais, ${ }^{1}$ com as seguintes propriedades:

a) Se $D \in \mathscr{D}$, então $\Delta(X) \subseteq D$;

b) Se $D_{1}, D_{2} \in \mathscr{D}$, então $D_{1} \cap D_{2} \in \mathscr{D}$;

c) Se $D \in \mathscr{D}$, então $D^{-1} \in \mathscr{D}$;

d) Para todo $D \in \mathscr{D}$, existe $E \in \mathscr{D}$ tal que $E \circ E \subseteq D$;

e) Se $D \in \mathscr{D}$ e $D \subseteq E \subseteq X \times X$, então $E \in \mathscr{D}$.

Note que uma estrutura uniforme $\mathscr{D}$ sobre um conjunto não vazio $X$ é um filtro sobre $X \times X$. Isso segue imediatamente das propriedades b) e e) da definição 3.1.1 e da observação de que $\emptyset \notin \mathscr{D}$ (pois $\Delta(X) \nsubseteq \emptyset$ ). Observe ainda que $D \subseteq D \circ D$ para todo $D \in \mathscr{D}$. Isso é uma consequência direta da propriedade a). Com efeito, para todo $D \in \mathscr{D}$ e todo $(x, y) \in X \times X$,

$$
(x, y) \in D \Rightarrow(x, x),(x, y) \in D \Rightarrow(x, y) \in D \circ D .
$$

Dada uma uniformidade $\mathscr{D}$ sobre um conjunto $X$, dizemos que os pontos $x, y \in X$ são $D$-próximos em relação a $\mathscr{D}$ se existir um $D \in \mathscr{D}$ tal que $(x, y) \in D$. Analogamente, se todos os pontos de um subconjunto $A \subseteq X$ forem $D$-próximos (isto é, se $A \times A \subseteq D$ ), diremos que $A$ é $D-$ pequeno.

Observe que, pela propriedade a) da definição 3.1.1, cada ponto $x \in X$ é $D$-próximo a si mesmo para todo $D \in \mathscr{D}$. A propriedade c) nos diz que um ponto $x$ é $D$-próximo a um ponto $y$ se, e somente se, $y$ for $D$-próximo a $x$. Já a propriedade d) garante que, para cada $D \in \mathscr{D}$, é possível encontrar $E \in \mathscr{D}$ com, no máximo, metade do "tamanho" de $D$. E, por fim, a propriedade e) afirma que, se $x, y \in X$ forem $D$-próximos e $D \subseteq E \subseteq X \times X$, então $x$ e $y$ serão, também, $E$-próximos (o que é, digamos, bastante razoável).

Essa nomenclatura (e, portanto, também a sua interpretação), tem sua origem no caso particular de uma uniformidade induzida por uma pseudométrica, o qual discutiremos mais adiante. Neste ponto, dar uma olhada na figura 3.3, na página 36, talvez ajude o leitor a entender melhor os comentários feitos no parágrafo anterior.

DefiniÇÃo 3.1.2. Dadas estruturas uniformes $\mathscr{D}_{1}$ e $\mathscr{D}_{2}$ sobre um mesmo conjunto $X$, dizemos que $\mathscr{D}_{1}$ é mais fina que $\mathscr{D}_{2}$ se $\mathscr{D}_{2} \subseteq \mathscr{D}_{1}$.

O conceito de estrutura uniforme motiva a seguinte definição:

\footnotetext{
${ }^{1}$ Em virtude da propriedade a) da definição 3.1.1.
} 
DeFINIÇÃo 3.1.3 (ESPAÇO UNIFORME). Um espaço uniforme é um par $(X, \mathscr{D})$, em que $X$ é um conjunto não vazio e $\mathscr{D}$ é uma estrutura uniforme sobre $X$.

Seja $\mathscr{D}$ uma estrutura uniforme sobre um conjunto $X$. Ao examinarmos a propriedade e) da definição 3.1.1, podemos ficar com a impressão de que, de algum modo, $\mathscr{D}$ possui "conjuntos em excesso". Isso nos sugere buscar uma subcoleção (preferencialmente, menor) de $\mathscr{D}$ a partir da qual seja possível estudar as suas propriedades. Nesse sentido, uma possibilidade seria obter uma subcoleção de $\mathscr{D}$ que nos permitisse recuperar os elementos de $\mathscr{D}$ por meio da propriedade e). Essa é a ideia por trás do conceito de base, o qual introduziremos a seguir.

DeFINIÇÃo 3.1.4 (BASE). Sejam $X$ um conjunto não vazio e $\mathscr{D}$ uma estrutura uniforme sobre $X$. Dizemos que uma subcoleção $\mathscr{B} \subseteq \mathscr{D}$ é uma base de $\mathscr{D}$ (ou que $\mathscr{B}$ gera $\mathscr{D}$ ) se cada $D \in \mathscr{D}$ contém algum elemento de $\mathscr{B}$.

OBSERVAÇÃo 3.1.5. Dadas uma uniformidade $\mathscr{D}$ sobre um conjunto $X$ e uma base $\mathscr{B}$ de $\mathscr{D}$, decorre diretamente da definição de base e da propriedade e) da definição 3.1.1 que

$$
\mathscr{D}=\{D \subseteq X \times X: \exists B \in \mathscr{B} \text { t.q. } B \subseteq D\} .
$$

A partir da observação 3.1.5, podemos derivar um resultado simples, mas importante, o qual enunciaremos a seguir. A demonstração é direta, e decorre imediatamente da observação 3.1.5.

Proposição 3.1.6. Sejam $\mathscr{D}_{1}$ e $\mathscr{D}_{2}$ uniformidades sobre um mesmo conjunto $X$. Nessas condições, $\mathscr{D}_{1} \subseteq \mathscr{D}_{2}$ se, e somente se, existem bases $\mathscr{B}_{1}$ e $\mathscr{B}_{2}$ de $\mathscr{D}_{1}$ e de $\mathscr{D}_{2}$, respectivamente, tais que cada elemento de $\mathscr{B}_{1}$ contém um elemento de $\mathscr{B}_{2}$.

A proposição 3.1.6 nos permite mostrar a igualdade entre duas uniformidades sobre um mesmo conjunto apenas trabalhando com os elementos de suas respectivas bases. Essa abordagem será útil muitas vezes ao longo deste capítulo.

Note que toda uniformidade é, trivialmente, uma base de si mesma. Disso resulta, em particular, que toda uniformidade admite, ao menos, uma base. Isso nos leva a considerar o problema inverso:

Como saber se uma dada subcoleção não vazia $\mathscr{B} \subseteq \mathcal{P}(X \times X)$ é base para alguma estrutura uniforme sobre $X$ ?

Segue da definição de base e da observação 3.1.5 que isso ocorre se, e somente se, a coleção

$$
\mathscr{D}:=\{D \subseteq X \times X: \exists B \in \mathscr{B} \text { t.q. } B \subseteq D\}
$$

for uma uniformidade sobre $X$ (e, em caso afirmativo, $\mathscr{B}$ é uma base de $\mathscr{D}$ ). A partir dessa observação, pode-se demonstrar a proposição abaixo, que fornece uma condição necessária e suficiente para que $\mathscr{B}$ gere uma uniformidade sobre $X$. A demonstração é simples e fica como exercício.

Proposição 3.1.7. Seja $X$ um conjunto não vazio. Nessas condições, uma coleção não vazia $\mathscr{B} \subseteq \mathcal{P}(X \times X)$ será base de uma uniformidade sobre $X$ se, e somente se, possuir as seguintes propriedades:

a) $S e B \in \mathscr{B}$, então $\Delta(X) \subseteq B$;

b) Para quaisquer $B_{1}, B_{2} \in \mathscr{B}$, existe $E \in \mathscr{B}$ tal que $E \subseteq B_{1} \cap B_{2}$;

c) Para todo $B \in \mathscr{B}$, existe $E \in \mathscr{B}$ tal que $E \subseteq B^{-1}$; 
d) Para todo $B \in \mathscr{B}$, existe $E \in \mathscr{B}$ tal que $E \circ E \subseteq B$.

Note que a coleção dos subconjuntos simétricos de uma estrutura uniforme $\mathscr{D}$ sobre um conjunto não vazio $X$ é uma base de $\mathscr{D}$. De fato, dado $D \in \mathscr{D}$, temos que $D^{-1} \in \mathscr{D}$ e, portanto, $E=D \cap D^{-1} \in \mathscr{D}$. Por outro lado, é imediato ver que $E$ é simétrico e que $E \subseteq D$.

Exemplos 3.1.8. Nos itens a) e b), a seguir, consideremos um conjunto $X$ não vazio.

a) A coleção $\mathscr{D}:=\{X \times X\}$ é uma uniformidade sobre $X$ conhecida como uniformidade trivial.

b) A coleção $\mathscr{D}:=\{D \subseteq X \times X: \Delta \subseteq D\}$ é uma uniformidade sobre $X$ denominada uniformidade discreta. Note que $\mathscr{B}:=\{\Delta\}$ é uma base de $\mathscr{D}$.

c) A uniformidade usual sobre $\mathbb{R}$ tem como base a coleção $\mathscr{B}_{1}:=\left\{D_{\varepsilon}: \varepsilon>0\right\}$, em que, para cada $\varepsilon>0$,

$$
D_{\varepsilon}:=\left\{(x, y) \in \mathbb{R}^{2}:|x-y|<\varepsilon\right\} .
$$

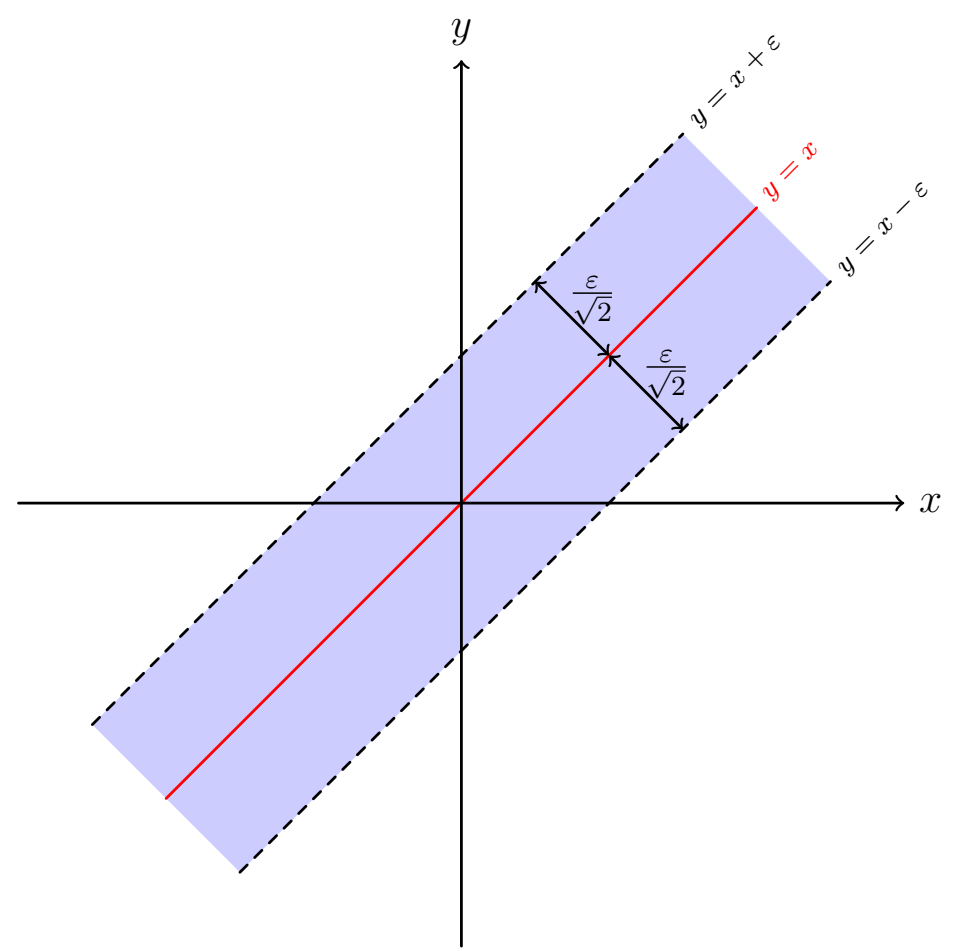

Figura 3.1: Representação no plano de um elemento de $\mathscr{B}_{1}$.

Vamos postergar, por hora, a demonstração de que $\mathscr{B}_{1}$ é, de fato, base para uma uniformidade sobre $\mathbb{R}$. Isso seguirá de um resultado mais geral, que será provado um pouco mais adiante. O nome uniformidade usual decorre do fato de que, como veremos, essa uniformidade está associada à metrica usual sobre $\mathbb{R}$.

d) A coleção $\mathscr{B}_{2}:=\left\{D_{a}: a \in \mathbb{R}\right\}$, em que, para cada $a \in \mathbb{R}$,

$$
D_{a}:=\Delta \cup\left\{(x, y) \in \mathbb{R}^{2}: x>a \text { e } y>a\right\}
$$

é outro exemplo de base para uma uniformidade sobre $\mathbb{R}$.

Demonstração. Para mostrarmos que $\mathscr{B}_{2}$ de fato gera uma uniformidade sobre $\mathbb{R}$, basta verificarmos que $\mathscr{B}_{2}$ satisfaz as condições a), b), c) e d) da proposição 3.1.7. 


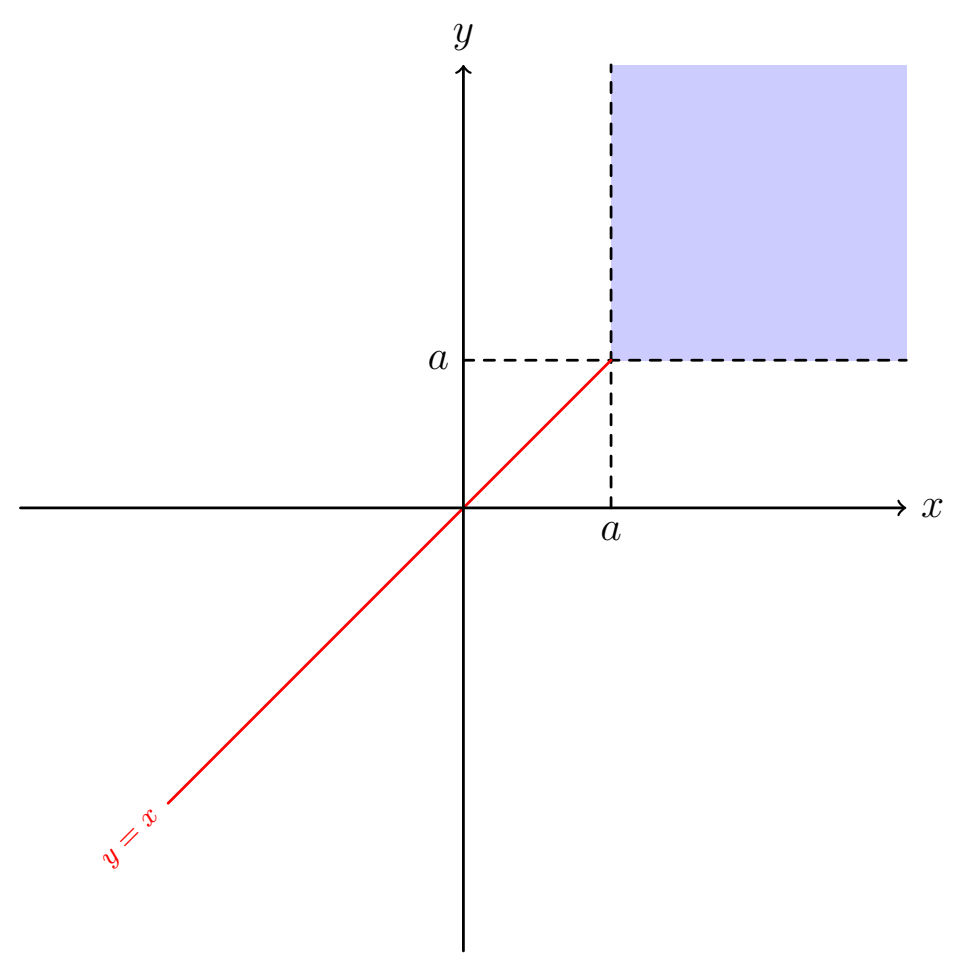

Figura 3.2: Representação no plano de um elemento de $\mathscr{B}_{2}$.

Seja $a \in \mathbb{R}$ dado. Note que $\Delta \subseteq D_{a}$ (por definição). Além disso, para todo $(x, y) \in \mathbb{R} \times \mathbb{R}$, temos que

$$
(x, y) \in D_{a} \Leftrightarrow x=y \text { ou }(x>a \text { e } y>a) \Leftrightarrow(y, x) \in D_{a} .
$$

Disso concluímos que $D_{a}=\left(D_{a}\right)^{-1}$.

A seguir, mostraremos que $D_{a} \circ D_{a} \subseteq D_{a}$. Como também vale a inclusão oposta (pois $\Delta \subseteq D_{a}$ ), disso resultará que $D_{a}=D_{a} \circ D_{a}$.

Seja $(x, y) \in D_{a} \circ D_{a}$ um ponto arbitrário. Por definição, disso segue que existe $z \in \mathbb{R}$ tal que $(x, z),(z, y) \in D_{a}$. Observe que

$$
(x, z) \in D_{a} \Rightarrow x=z \text { ou }(x>a \text { e } z>a) .
$$

Analogamente,

$$
(z, y) \in D_{a} \Rightarrow z=y \text { ou }(z>a \text { e } y>a)
$$

Logo, temos quatro possibilidades: ou $x=z=y$; ou $x=z$ e $z, y>a$; ou $z=y$ e $x, z>a$; ou $x, z, y>a$. No primeiro caso, temos que $x=y$ e, nos demais, que $x, y>a$. Dessa forma, em qualquer um dos casos, vale que $(x, y) \in D_{a}$.

Por fim, mostremos que $\mathscr{B}_{2}$ satisfaz b). Para isso, tomemos $a_{1}, a_{2} \in \mathbb{R}$ quaisquer. Seja $M:=\max \left\{a_{1}, a_{2}\right\}$. Note que, para todo $(x, y) \in \mathbb{R}^{2}$, temos que

$(x, y) \in D_{a_{1}} \cap D_{a_{2}} \Leftrightarrow x=y$ ou $\left(x, y>a_{1}\right.$ e $\left.x, y>a_{2}\right) \Leftrightarrow x=y$ ou $x, y>M \Leftrightarrow(x, y) \in D_{M}$.

E, portanto, $D_{M}=D_{a_{1}} \cap D_{a_{2}}$.

Dados um espaço uniforme $\left(X, \mathscr{D}_{X}\right)$ e um subconjunto $A \subseteq X$ não vazio, será que podemos produzir uma uniformidade sobre $A$ a partir de $\mathscr{D}_{X}$ ? A resposta a essa pergunta é afirmativa e, no próximo exemplo, mostraremos como fazer isso. Aqueles que estão familiarizados com 
topologia provavelmente notarão a semelhança entre as definições dessa "uniformidade de subespaço" e da topologia de subespaço de um espaço topológico. Na verdade, mostraremos, mais adiante, que essa semelhança não é mera coincidência.

Exemplo 3.1.9 (SubespaÇo uniforme). Sejam $\left(X, \mathscr{D}_{X}\right)$ um espaço uniforme e $A \subseteq X$ um subconjunto não vazio. Nessas condições, a coleção

$$
\mathscr{D}_{A}:=\left\{D \cap(A \times A): D \in \mathscr{D}_{X}\right\}
$$

é uma uniformidade sobre $A$. Dizemos que o par $\left(A, \mathscr{D}_{A}\right)$ é um subespaço uniforme de $\left(X, \mathscr{D}_{X}\right)$.

Demonstração. Primeiramente, note que

$$
\Delta(A)=\Delta(X) \cap(A \times A) \subseteq D \cap(A \times A)
$$

para todo $D \in \mathscr{D}_{X}$. Dessa forma, $\mathscr{D}_{A}$ satisfaz a).

Observe ainda que, dados $D_{1}, D_{2} \in \mathscr{D}_{X}$, temos que $D_{1} \cap D_{2} \in \mathscr{D}_{X}$ e que

$$
\left[D_{1} \cap(A \times A)\right] \cap\left[D_{2} \cap(A \times A)\right]=\left(D_{1} \cap D_{2}\right) \cap(A \times A) .
$$

Isso mostra que $\mathscr{D}_{A}$ satisfaz b).

Para mostrarmos que $\mathscr{D}_{A}$ satisfaz as demais condições, tomemos um conjunto $D^{\prime} \in \mathscr{D}_{A}$ qualquer. Por definição, disso segue que existe $D \in \mathscr{D}_{X}$ tal que $D^{\prime}=D \cap(A \times A)$.

Note que

$$
\left(D^{\prime}\right)^{-1}=(D \cap(A \times A))^{-1}=D^{-1} \cap(A \times A) .
$$

Como $D^{-1} \in \mathscr{D}_{X}$, disso resulta que $\left(D^{\prime}\right)^{-1} \in \mathscr{D}_{A}$.

Vamos, agora, provar que existe $F^{\prime} \in \mathscr{D}_{A}$ tal que $F^{\prime} \circ_{A} F^{\prime} \subseteq D^{\prime}$ (sendo $\circ_{A}$ a restrição de $\circ$ a $\mathcal{P}(A \times A) \times \mathcal{P}(A \times A))$. Para isso, observe, inicialmente, que, por d), podemos fixar $F \in \mathscr{D}_{X}$ tal que $F \circ F \subseteq D$. E, além disso, é fácil ver que

$$
\begin{aligned}
{[F \cap(A \times A)] \circ_{A}[F \cap(A \times A)] } & :=\{(x, y) \in A \times A: \exists z \in A \text { t.q. }(x, z),(z, y) \in F \cap(A \times A)\} \\
& \subseteq\{(x, y) \in A \times A: \exists z \in X \text { t.q. }(x, z),(z, y) \in F\} \\
& =(F \circ F) \cap(A \times A) \subseteq D \cap(A \times A)=D^{\prime} .
\end{aligned}
$$

Dessa forma, se tomarmos $F^{\prime}:=F \cap(A \times A)$, teremos que $F^{\prime} \in \mathscr{D}_{A}$ e que $F^{\prime} \circ_{A} F^{\prime} \subseteq D^{\prime}$.

Por fim, precisamos, ainda, mostrar que, se $G^{\prime} \subseteq A \times A$ é tal que $D^{\prime} \subseteq G^{\prime}$, então $G^{\prime} \in \mathscr{D}_{A}$. Dado $G^{\prime}$ nas condições acima, tomemos $G:=G^{\prime} \cup D$. Note que

$$
D=(D \cap(A \times A)) \cup D=D^{\prime} \cup D \subseteq G^{\prime} \cup D=G \subseteq X \times X .
$$

Portanto, segue de e) que $G \in \mathscr{D}_{X}$. Por fim, como

$$
G \cap(A \times A)=\left(G^{\prime} \cup D\right) \cap(A \times A)=\left(G^{\prime} \cap(A \times A)\right) \cup(D \cap(A \times A))=G^{\prime} \cup D^{\prime}=G^{\prime},
$$

disso resulta que $G^{\prime} \in \mathscr{D}_{A}$.

Como $D^{\prime} \in \mathscr{D}_{A}$ é arbitrário, segue do que mostramos acima que $\mathscr{D}_{A}$ também satisfaz c), d) e e) e, portanto, é uma uniformidade sobre $A$.

OBSERVAÇÃo 3.1.10. Sejam $\left(X, \mathscr{D}_{X}\right)$ e $\left(A, \mathscr{D}_{A}\right)$ como no exemplo 3.1.9. Nessas condições, se $\mathscr{B}_{X}$ for uma base de $\mathscr{D}_{X}$, é fácil ver que

$$
\mathscr{B}_{A}:=\left\{B \cap(A \times A): B \in \mathscr{B}_{X}\right\}
$$


será uma base de $\mathscr{D}_{A}$.

O próximo exemplo é de fundamental importância para o desenvolvimento deste trabalho, e será utilizado recorrentemente nas próximas seções. Por essa razão, merece ser analisado separadamente e em detalhes. Mas, primeiro, vamos definir uma notação conveniente, a qual será utilizada ao longo de todo o trabalho.

DefiniÇão 3.1.11. Sejam $X$ um conjunto não vazio e $d$ uma pseudométrica sobre $X$. Para cada $\varepsilon>0$, denotaremos por $D_{\varepsilon}^{d}$ (ou, equivalentemente, por $E_{\varepsilon}^{d}$ ou $F_{\varepsilon}^{d}$ ) o conjunto

$$
\{(x, y) \in X \times X: d(x, y)<\varepsilon\} .
$$

Exemplo 3.1.12 (ESTRUtura Uniforme induZida Por uma PSEudométrica). Sejam $X$ um conjunto não vazio e $d$ uma pseudométrica sobre $X$. Nessas condições, a coleção

$$
\mathscr{B}_{d}:=\left\{D_{\varepsilon}^{d}: \varepsilon>0\right\}
$$

é base de uma uniformidade sobre $X$.

(Com frequência, referir-nos-emos a essa uniformidade como uniformidade induzida por d sobre $X$.)

Demonstração. Vamos verificar que $\mathscr{B}_{d}$ satisfaz as condições a), b), c) e d) da proposição 3.1.7, a partir do que seguirá de 3.1.7 que $\mathscr{B}_{d}$ gera uma uniformidade sobre $X$.

Seja $\varepsilon>0$ dado. Observe que $\Delta \subseteq D_{\varepsilon}^{d}$, pois $d(x, x)=0<\varepsilon$ para todo $x \in X$. Além disso, segue da simetria de $d$ que, para todo $(x, y) \in X \times X$,

$$
(x, y) \in D_{\varepsilon}^{d} \Leftrightarrow d(x, y)<\varepsilon \Leftrightarrow d(y, x)<\varepsilon \Leftrightarrow(y, x) \in D_{\varepsilon}^{d}
$$

e, portanto, $\left(D_{\varepsilon}^{d}\right)^{-1}=D_{\varepsilon}^{d}$.

A seguir, provaremos que $D_{\frac{\varepsilon}{2}}^{d} \circ D_{\frac{\varepsilon}{2}}^{d} \subseteq D_{\varepsilon}^{d}$. Para isso, basta mostrarmos que todo $(x, y)$ em $D_{\frac{\varepsilon}{2}}^{d} \circ D_{\frac{\varepsilon}{2}}^{d}$ pertence a $D_{\varepsilon}^{d}$. Seja $(x, y) \in D_{\frac{\varepsilon}{2}}^{d} \circ D_{\frac{\varepsilon}{2}}^{d}$ um ponto qualquer. Por definição, disso segue que existe $z \in X$ tal que $(x, z),(z, y) \in D_{\frac{\varepsilon}{2}}^{d}$. Mas:

$$
(x, z),(z, y) \in D_{\frac{\varepsilon}{2}}^{d} \Rightarrow d(x, z), d(z, y)<\frac{\varepsilon}{2} \Rightarrow d(x, y) \leq d(x, z)+d(z, y)<\frac{\varepsilon}{2}+\frac{\varepsilon}{2}=\varepsilon .
$$

E, sendo assim, $(x, y) \in D_{\varepsilon}^{d}$.

Como $\varepsilon>0$ é arbitrário, disso resulta que $\mathscr{B}_{d}$ satisfaz a), c) e d). Por fim, vamos mostrar que $\mathscr{B}_{d}$ também satisfaz b).

$\operatorname{Dados} \varepsilon_{1}, \varepsilon_{2}>0$, tomemos $\varepsilon=\min \left\{\varepsilon_{1}, \varepsilon_{2}\right\}$. Note que, para todo $(x, y) \in X \times X$,

$$
(x, y) \in D_{\varepsilon}^{d} \Leftrightarrow d(x, y)<\varepsilon \Leftrightarrow d(x, y)<\varepsilon_{1}, \varepsilon_{2} \Leftrightarrow(x, y) \in D_{\varepsilon_{1}}^{d} \cap D_{\varepsilon_{2}}^{d} .
$$

Disso concluímos que $D_{\varepsilon}^{d}=D_{\varepsilon_{1}}^{d} \cap D_{\varepsilon_{2}}^{d}$. E, com esse resultado, encerramos a demonstração.

NotAÇÃo: A uniformidade induzida por uma pseudométrica $d$ sobre um conjunto $X$ será denotada por $\mathscr{D}_{d}$.

OBSERVAÇÃo 3.1.13. Dados um espaço pseudométrico $(X, d)$ e um subconjunto não vazio $A \subseteq$ $X$, não é difícil mostrar que $\left(\mathscr{D}_{d}\right)_{A}=\mathscr{D}_{\left.d\right|_{A \times A}}$. Com efeito, isso é uma consequência imediata da observação 3.1.10, uma vez que, para cada $\varepsilon>0$,

$$
D_{\varepsilon}^{d} \cap(A \times A)=\{(x, y) \in A \times A: d(x, y)<\varepsilon\}=D_{\varepsilon}^{\left.d\right|_{A \times A}} .
$$


A figura 3.3 ilustra uma vizinhança diagonal genérica da uniformidade induzida pela métrica usual de $\mathbb{R}$.

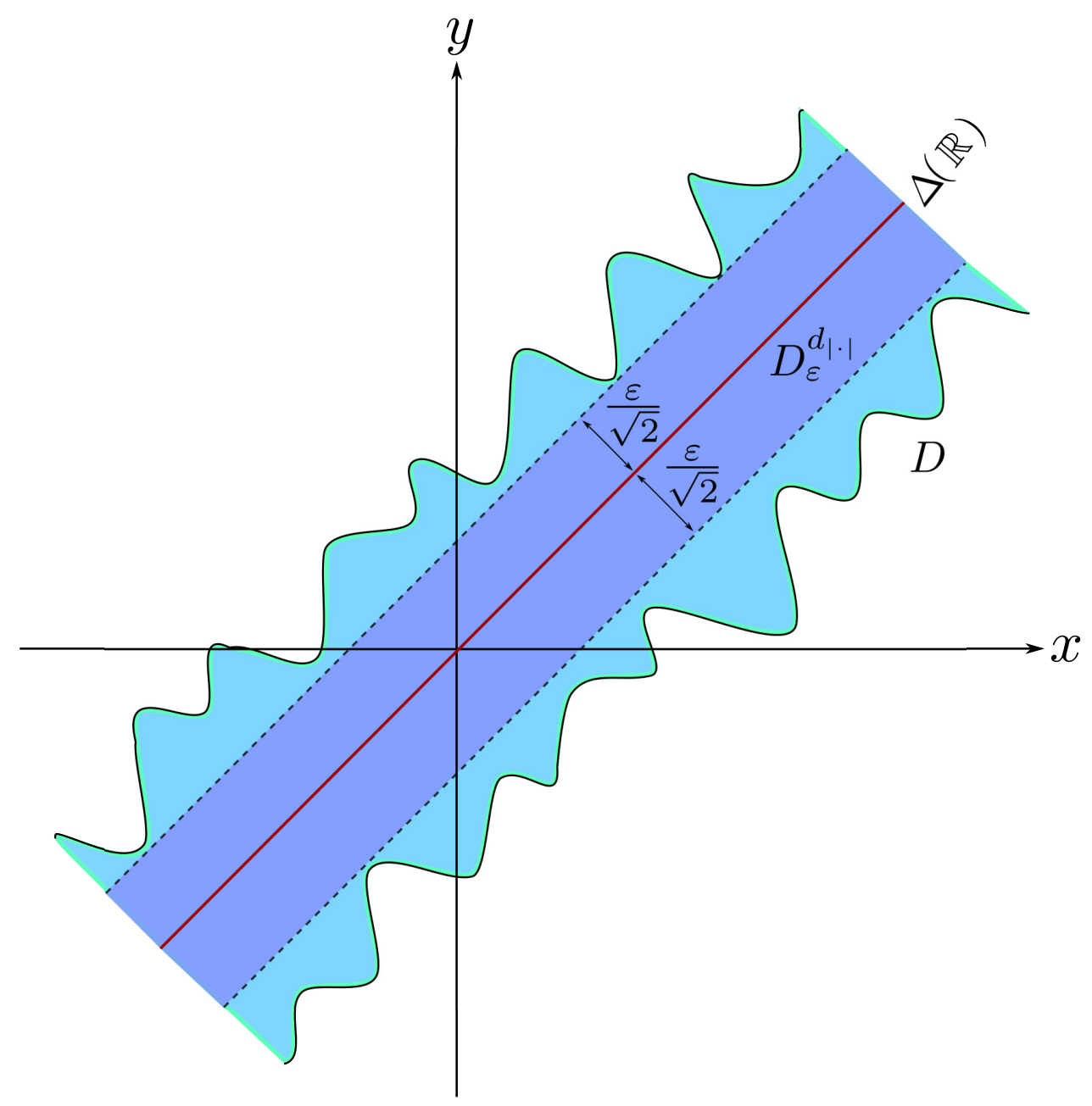

Figura 3.3: Vizinhança diagonal da uniformidade usual sobre $\mathbb{R}$.

Após dar uma boa olhada na figura 3.3, talvez seja uma boa hora para reler os comentários que sucedem a definição de uniformidade, os quais, à luz do exemplo anterior, parecerão, agora, muito mais claros.

DEFINIÇÃo 3.1.14. As uniformidades que podem ser geradas a partir de pseudométricas são chamadas pseudometrizáveis.

OBSERVAÇÃo 3.1.15. Note que, se $d$ é uma pseudométrica sobre um conjunto $X$, então $2 d$ também é uma pseudométrica sobre $X$ e as estruturas uniformes geradas por $d$ e $2 d$ coincidem. Disso resulta que pseudométricas diferentes sobre um mesmo conjunto podem gerar a mesma estrutura uniforme e, portanto, uma estrutura uniforme representa, de fato, menos estrutura sobre um conjunto do que uma pseudométrica.

A proposição a seguir estabelece uma condição suficente para que duas pseudométricas sobre um mesmo conjunto $X$ deem origem a uma mesma estrutura uniforme sobre $X$.

Proposição 3.1.16. Sejam $d_{1}$ e $d_{2}$ duas pseudométricas sobre um mesmo conjunto $X, e$ suponhamos que existam funções $\varphi_{1}:\left[0,+\infty\left[\longrightarrow\left[0,+\infty\left[\right.\right.\right.\right.$ e $\varphi_{2}:[0,+\infty[\longrightarrow[0,+\infty[$ continuas em $0, \operatorname{com} \varphi_{1}(0)=\varphi_{2}(0)=0$, e tais que, para todo $(x, y) \in X \times X, d_{1}(x, y) \leq \varphi_{2}\left(d_{2}(x, y)\right) e$ $d_{2}(x, y) \leq \varphi_{1}\left(d_{1}(x, y)\right)$. Nessas condições, as uniformidades geradas por $d_{1}$ e por $d_{2}$ coincidem. 
Demonstração. Sejam $\mathscr{D}_{1}$ e $\mathscr{D}_{2}$ as respectivas uniformidades induzidas por $d_{1}$ e por $d_{2}$. Vamos mostrar que, para cada $\varepsilon>0$, existe $\delta>0$ tal que $D_{\delta}^{d_{2}} \subseteq D_{\varepsilon}^{d_{1}}$. Como $\mathscr{B}_{1}:=\left\{D_{\varepsilon}^{d_{1}}: \varepsilon>0\right\}$ e $\mathscr{B}_{2}:=\left\{D_{\delta}^{d_{2}}: \delta>0\right\}$ são bases de $\mathscr{D}_{1}$ e de $\mathscr{D}_{2}$, respectivamente, disso seguirá que $\mathscr{D}_{1} \subseteq \mathscr{D}_{2}$ (pela proposição 3.1.6).

Seja $\varepsilon>0$ dado. Como $\varphi_{2}$ é contínua em 0 e $\varphi_{2}(0)=0$, podemos fixar $\delta>0$ tal que $\varphi_{2}\left(d_{2}(x, y)\right)<\varepsilon$ para todos $x, y \in X$ tais que $d_{2}(x, y)<\delta$. Por sua vez, como $d_{1}(x, y) \leq$ $\varphi_{2}\left(d_{2}(x, y)\right)$ para todos $x, y \in X$, disso segue que, para todo $(x, y) \in X \times X$,

$$
(x, y) \in D_{\delta}^{d_{2}} \Rightarrow d_{2}(x, y)<\delta \Rightarrow \varphi_{2}\left(d_{2}(x, y)\right)<\varepsilon \Rightarrow d_{1}(x, y)<\varepsilon \Rightarrow(x, y) \in D_{\varepsilon}^{d_{1}},
$$

a partir do que concluímos que $D_{\delta}^{d_{2}} \subseteq D_{\varepsilon}^{d_{1}}$.

Como $\varepsilon>0$ é arbitrário, isso completa a prova de que $\mathscr{D}_{1} \subseteq \mathscr{D}_{2}$ (veja a proposição 3.1.6). Invertendo os papéis de 1 e de 2 e repetindo o argumento, obtemos a inclusão oposta.

Vejamos, agora, um exemplo de aplicação da proposição 3.1.16, o qual enunciaremos sob a forma de um corolário.

Corolário 3.1.17. Seja d $d_{1}$ uma pseudométrica sobre um conjunto $X$. Nessas condições, $d_{2}: X \times X \longrightarrow \mathbb{R}$ tal que $d_{2}(x, y)=\sqrt{d_{1}(x, y)}$ também é uma pseudométrica sobre $X$ e ambas induzem sobre $X$ a mesma uniformidade.

Demonstração. A demonstração de que $d_{2}$ é uma pseudométrica é um exercício simples, o qual é deixado a cargo do leitor.

Para a segunda parte da demonstração, consideremos $\varphi_{1}, \varphi_{2}:[0,+\infty[\longrightarrow[0,+\infty[$ tais que $\varphi_{1}(u)=\sqrt{u}$ e $\varphi_{2}(u)=u^{2}$. Observe que $\varphi_{1}$ e $\varphi_{2}$ são contínuas em 0 e que $\varphi_{1}(0)=\varphi_{2}(0)=0$. Além disso, para todo $(x, y) \in X \times X$, temos que

$$
d_{1}(x, y)=\left(\sqrt{d_{1}(x, y)}\right)^{2}=\left(d_{2}(x, y)\right)^{2}=\varphi_{2}\left(d_{2}(x, y)\right)
$$

$\mathrm{e}$

$$
d_{2}(x, y)=\sqrt{d_{1}(x, y)}=\varphi_{1}\left(d_{1}(x, y)\right) .
$$

Logo, segue da proposição 3.1 .16 que $\mathscr{D}_{d_{1}}=\mathscr{D}_{d_{2}}$.

\subsection{A ESTRUtura UNIFORME À ESQUERDA DE UM GRUPo TOPOLÓGICO}

A seguir, vamos analisar, com particular atenção, o caso dos grupos topológicos. Como é bem conhecido, várias uniformidades podem ser definidas de maneira natural a partir da estrutura de um grupo topológico. Nesta seção, vamos nos concentrar em uma delas: a estrutura uniforme à esquerda. Deste ponto em diante, salvo menção em contrário, sempre denotaremos por $e$ o elemento neutro de um grupo topológico e por $\mathcal{V}$ a coleção das vizinhanças de $e$ em relação à topologia do grupo.

No que segue, consideremos um grupo topológico $(G, \cdot, \tau)$.

Definição 3.2.1. Para cada $V \in \mathcal{V}$, denotaremos por $E_{V}$ o conjunto

$$
\left\{(x, y) \in G \times G: x^{-1} y \in V\right\} .
$$


A coleção $\left\{E_{V}: V \in \mathcal{V}\right\}$ será denotada por $\mathscr{L}$.

Proposição 3.2.2. A coleção $\mathscr{L}$ é base para uma uniformidade sobre $G$.

Demonstração. Vamos verificar que $\mathscr{L}$ satisfaz as condições da proposição 3.1 .7 e, portanto, gera uma uniformidade sobre $G$.

Inicialmente, observe que, se $V_{1}, V_{2} \in \mathcal{V}$, então $V_{1} \cap V_{2} \in \mathcal{V}$ e

$$
\begin{aligned}
E_{V_{1} \cap V_{2}}: & =\left\{(x, y) \in G \times G: x^{-1} y \in V_{1} \cap V_{2}\right\} \\
& =\left\{(x, y) \in G \times G: x^{-1} y \in V_{1}\right\} \cap\left\{(x, y) \in G \times G: x^{-1} y \in V_{2}\right\}=E_{V_{1}} \cap E_{V_{2}} .
\end{aligned}
$$

Isso mostra que $\mathscr{L}$ satisfaz b).

Para mostrarmos que $\mathscr{L}$ também satisfaz a), c) e d), consideremos uma vizinhança $V$ de $e$ qualquer. Como $x^{-1} x=e \in V$ para todo $x \in G$, podemos, prontamente, concluir que $\Delta \subseteq E_{V}$.

Seja $i: G \longrightarrow G$ tal que $i(x)=x^{-1}$. Como $i(e)=e, V$ é vizinhança de $e$ e $i$ é contínua em $e$, existe $U \in \tau$ tal que $e \in U$ e $i(U) \subseteq V$. Note que, para todo $(x, y) \in G \times G$,

$$
\begin{aligned}
(x, y) \in E_{U}^{-1} & \Rightarrow(y, x) \in E_{U} \Rightarrow y^{-1} x \in U \Rightarrow\left(y^{-1} x\right)^{-1} \in i(U) \Rightarrow x^{-1} y \in i(U) \Rightarrow x^{-1} y \in V \\
& \Rightarrow(x, y) \in E_{V}
\end{aligned}
$$

Disso resulta que $E_{U}^{-1} \subseteq E_{V}$.

A seguir, consideremos $m: G \times G \longrightarrow G$ tal que $m(x, y)=x y$. Como $m(e, e)=e, V$ é vizinhança de $e, m$ é contínua em $(e, e)$ e

$$
\mathscr{B}:=\left\{W_{1} \times W_{2}: W_{1}, W_{2} \in \tau\right\}
$$

é base da topologia produto induzida por $\tau$ sobre $G \times G$, existem $W_{1}$ e $W_{2}$ em $\tau$ tais que $(e, e) \in W_{1} \times W_{2}$ e $m\left(W_{1} \times W_{2}\right) \subseteq V$. Seja $W=W_{1} \cap W_{2}$. Note que $W \in \tau, e \in W$ e $m(W \times W) \subseteq m\left(W_{1} \times W_{2}\right) \subseteq V$.

Vamos mostrar que $E_{W} \circ E_{W} \subseteq E_{V}$. Para isso, tomemos $(x, y) \in E_{W} \circ E_{W}$ de modo arbitrário. Por definição, disso segue que existe $z \in G$ tal que $(x, z),(z, y) \in E_{W}$. Mas:

$$
\begin{aligned}
(x, z),(z, y) \in E_{W} & \Rightarrow x^{-1} z, z^{-1} y \in W \Rightarrow\left(x^{-1} z, z^{-1} y\right) \in W \times W \Rightarrow m\left(x^{-1} z, z^{-1} y\right) \in V \\
& \Rightarrow x^{-1} y \in V .
\end{aligned}
$$

Dessa forma, $(x, y) \in E_{V}$, e o resultado está provado.

Por fim, como $V \in \mathcal{V}$ é arbitrário, concluímos do que foi feito acima que $\mathscr{L}$ satisfaz a), c) e d).

DefiniçÃo 3.2.3 (Estrutura Uniforme À ESQUERDA). A uniformidade gerada por $\mathscr{L}$ recebe o nome de estrutura uniforme à esquerda ${ }^{2}$ (ou uniformidade à esquerda) associada a $(G, \cdot, \tau)$, e será denotada por $\mathcal{U}_{L}$.

O nome "estrutura uniforme à esquerda" decorre do fato de que, para todo $V \in \mathcal{V}, E_{V}$ é invariante à esquerda em $(G, \cdot)$. Com efeito, dados $V \in \mathcal{V}$ e $x, y, g \in G$, é imediato ver que

$$
(g x)^{-1}(g y)=\left(x^{-1} g^{-1}\right)(g y)=x^{-1}\left(g^{-1} g\right) y=x^{-1} e y=x^{-1} y .
$$

E, portanto,

$$
(x, y) \in E_{V} \Rightarrow x^{-1} y \in V \Rightarrow(g x)^{-1}(g y) \in V \Rightarrow(g x, g y) \in E_{V} .
$$

\footnotetext{
${ }^{2}$ Do inglês left-uniform structure.
} 
A proposição 3.2.2 tem o seguinte corolário:

Corolário 3.2.4. Se $\mathcal{U}$ for uma base local de e em $(G, \tau)$, então $\mathscr{B}_{\mathcal{U}}:=\left\{E_{U}: U \in \mathcal{U}\right\}$ será uma base de $\mathcal{U}_{L}$.

Demonstração. Seja $\mathcal{U}$ uma base local de $e$ em $(G, \tau)$. Inicialmente, observe que

$$
\mathcal{U} \subseteq \mathcal{V} \Rightarrow \mathscr{B}_{\mathcal{U}} \subseteq \mathscr{L} \Rightarrow \mathscr{B}_{\mathcal{U}} \subseteq \mathcal{U}_{L}
$$

Assim sendo, $\mathscr{B}_{\mathcal{U}}$ é uma subcoleção de $\mathcal{U}_{L}$.

Vamos, agora, mostrar que cada $D \in \mathcal{U}_{L}$ contém um elemento de $\mathscr{B}_{\mathcal{U}}$. Para isso, tomemos $D \in \mathcal{U}_{L}$ de modo arbitrário. Como $\mathscr{L}$ é base de $\mathcal{U}_{L}$, podemos fixar $V \in \mathcal{V}$ de modo que $E_{V} \subseteq D$. Analogamente, como $\mathcal{U}$ é base local de e e $V$ é vizinhança de $e$, podemos fixar $U \in \mathcal{U}$ tal que $U \subseteq V$. Mas:

$$
U \subseteq V \Rightarrow E_{U} \subseteq E_{V}
$$

E, como $E_{V} \subseteq D$, disso segue que $E_{U} \subseteq D$.

OBSERVAÇÃO 3.2.5. Além da estrutura uniforme à esquerda, outras uniformidades podem ser definidas de forma natural a partir da estrutura de $(G, \cdot, \tau)$. Um exemplo é a estrutura uniforme à direita, a qual denotaremos por $\mathcal{U}_{R}$, e que tem como base a coleção $\mathscr{R}:=\left\{R_{V}: V \in \mathcal{V}\right\}$, em que, para cada $V \in \mathcal{V}$,

$$
R_{V}:=\left\{(x, y) \in G \times G: y x^{-1} \in V\right\} .
$$

Observe que, para cada $V \in \mathcal{V}, R_{V}$ é invariante à direita em $(G, \cdot)$.

Quando $\mathcal{U}_{L}=\mathcal{U}_{R}$, dizemos que $(G, \cdot, \tau)$ tem estruturas uniformes equivalentes. Decorre diretamente das definições que todo grupo topológico abeliano tem estruturas uniformes equivalentes, e pode-se mostrar (de forma não tão trivial) que isso também ocorre no caso dos grupos topológicos compactos.

Uma subclasse particularmente importante de grupos topológicos é a dos grupos topológicos pseudometrizáveis, isto é, aqueles cuja topologia vem de uma pseudométrica. No que segue, vamos analisar essa subclasse à luz do que vimos sobre estruturas uniformes.

Após refletir um pouco a respeito dos grupos topológicos pseudometrizáveis, é natural que o leitor sinta-se tentado a levantar a seguinte questão:

Dado que, nesse caso, a topologia do grupo vem de uma pseudométrica, será que podemos garantir que o mesmo ocorre com a estrutura uniforme à esquerda?

A resposta a essa pergunta é negativa. Com efeito, é possível mostrar que um espaço uniforme é pseudometrizável se, e somente se, possui uma base enumerável. ${ }^{3}$ Observe que, todavia, isso não garante que todas as pseudométricas compatíveis com a topologia de um grupo topológico pseudometrizável sejam também compatíveis com sua estrutura uniforme à esquerda. Em verdade, não há, a priori, nenhum motivo para crermos que uma pseudométrica $d$, compatível com a topologia de um grupo $(G, \cdot)$, deva, necessariamente, induzir sobre $G$ a estrutura uniforme à esquerda. E, sendo assim, seria interessante estabelecer condições suficientes para que isso ocorra.

Para tanto, inicialmente observe que a coleção

$$
\mathscr{L}:=\left\{E_{V}: V \in \mathcal{V}\right\},
$$

\footnotetext{
${ }^{3}$ Por fugir um pouco do objetivo deste trabalho, não apresentaremos aqui a demonstração desse resultado, a qual pode ser encontrada, por exemplo, na referência [Wil70].
} 
a qual é, por definição, uma base de $\mathcal{U}_{L}$, é formada por conjuntos invariantes à esquerda. Com sorte, após alguns momentos de reflexão, isso conduza o leitor a conjecturar que uma boa candidata a induzir sobre $G$ a estrutura uniforme à esquerda seja, justamente, uma pseudométrica $d$ sobre $G$ tal que a coleção

$$
\mathscr{B}_{d}:=\left\{D_{\varepsilon}^{d}: \varepsilon>0\right\}
$$

é, também, constituída por conjuntos invariantes à esquerda. Em termos da pseudométrica, isso significa dizer que, para todo $\varepsilon>0$ e todos $x, y, \lambda \in G$,

$$
d(x, y)<\varepsilon \Rightarrow d(\lambda x, \lambda y)<\varepsilon .
$$

E, por sua vez, não é difícil mostrar que isso ocorre se, e somente se, $d(\lambda x, \lambda y)=d(x, y)$ quaisquer que sejam $x, y, \lambda \in G$. Pseudométricas com essa propriedade são ditas invariantes à esquerda. A classe das pseudométricas invariantes à esquerda em um dado grupo desempenhará um papel bastante importante nos capítulos seguintes. Por essa razão, vamos, agora, formalizar esse conceito, apresentando-o em forma de definição.

DEFINIÇÃo 3.2.6. Dado um grupo topológico $(G, \cdot, \tau)$, dizemos que uma pseudométrica $d$ sobre $G$ é invariante à esquerda em $(G, \cdot)$ se $d(g x, g y)=d(x, y)$ quaisquer que sejam $g, x, y \in$ $G$.

Vejamos, a seguir, um exemplo importante de métrica invariante à esquerda em um grupo. Exemplo 3.2.7. Sejam $(G, \cdot)$ um grupo finitamente gerado e $\mathcal{F}$ uma família finita de geradores de $(G, \cdot)$ tal que, para todo $g \in \mathcal{F}, g^{-1} \in \mathcal{F}$. Nessas condições, a função $d: G \times G \longrightarrow \mathbb{R}$ tal que

$$
d(x, y)= \begin{cases}0, & \text { se } x=y \\ \min \left\{k \in \mathbb{N}: \exists a_{1}, \ldots, a_{k} \in \mathcal{F} \text { t.q. } x^{-1} y=a_{1} \cdot \ldots \cdot a_{k}\right\}, & \text { se } x \neq y\end{cases}
$$

é uma métrica sobre $G$ invariante à esquerda em $(G, \cdot)$.

Demonstração. Vamos começar mostrando que d é uma métrica sobre G. Para tanto, precisamos verificar que $d$ satisfaz as condições expressas na definição 2.3.1.

Inicialmente, observe que, por definição,

$$
d(x, y) \geq 0 \text { e } d(x, y)=0 \Leftrightarrow x=y
$$

para todos $x, y \in G$. Assim sendo, basta provarmos que $d$ é simétrica e satisfaz a desigualdade triangular. Para isso, tomemos $x, y \in G$ de modo arbitrário. O caso em que $x=y$ é óbvio. E, sendo assim, podemos supor que $x \neq y$. Observe que, para todo $k \in \mathbb{N}$ e todos $a_{1}, \ldots, a_{k} \in \mathcal{F}$,

$$
x^{-1} y=a_{1} \cdot \ldots \cdot a_{k} \Leftrightarrow y^{-1} x=\left(x^{-1} y\right)^{-1}=\left(a_{1} \cdot \ldots \cdot a_{k}\right)^{-1}=a_{k}{ }^{-1} \cdot \ldots \cdot a_{1}^{-1} .
$$

Como $k \in \mathbb{N}$ é arbitrário e $a^{-1} \in \mathcal{F}$ para todo $a \in \mathcal{F}$, disso concluímos que $d(x, y)=d(y, x)$.

Tomemos, agora, um ponto $z \in G$ qualquer, e vamos mostrar que

$$
d(x, y) \leq d(x, z)+d(z, y) .
$$

Isso é óbvio se $z=x$ ou $z=y$, de forma que podemos nos restringir ao caso em que $x \neq z \neq y$. Para isso, definamos $m:=d(x, z)$ e $n:=d(z, y)$, e fixemos $b_{1}, \ldots, b_{m}, c_{1}, \ldots, c_{n} \in \mathcal{F}$ tais que

$$
x^{-1} z=b_{1} \cdot \ldots \cdot b_{m} \text { e } z^{-1} y=c_{1} \cdot \ldots \cdot c_{n} .
$$


Tendo em vista que

$$
x^{-1} y=\left(x^{-1} z\right)\left(z^{-1} y\right)=\left(b_{1} \cdot \ldots \cdot b_{m}\right)\left(c_{1} \cdot \ldots \cdot c_{n}\right),
$$

segue diretamente da definição de $d$ que

$$
d(x, y)=\min \left\{k \in \mathbb{N}: \exists a_{1}, \ldots, a_{k} \in \mathcal{F} \text { t.q. } x^{-1} y=a_{1} \cdot \ldots \cdot a_{k}\right\} \leq m+n=d(x, z)+d(z, y) .
$$

Isso conclui a prova de que $d$ é uma métrica sobre $G$. E, uma vez que, para todo $\lambda \in G$ e todo $(x, y) \in G \times G$, vale que

$$
(\lambda x)^{-1}(\lambda y)=\left(x^{-1} \lambda^{-1}\right)(\lambda y)=x^{-1} y,
$$

fica claro que $d$ é invariante à esquerda.

DEFINIÇÃo 3.2.8. A métrica definida no exemplo 3.2.7 é conhecida como métrica da palavra associada ao grupo $(G, \cdot)$ e ao conjunto gerador $\mathcal{F}^{4}$

Após esta pequena digressão, podemos, enfim, retornar ao problema inicial. Isto é: determinar condições suficientes para que uma pseudométrica $d$, compatível com a topologia de um grupo $(G, \cdot)$, induza sobre $G$ a estrutura uniforme à esquerda. A próxima proposição enuncia um importante resultado a esse respeito.

Proposição 3.2.9. Sejam $(G, \cdot, \tau)$ um grupo topológico e d uma pseudométrica compatível sobre $G$. Nessas condições, se d for invariante à esquerda em $(G, \cdot)$, então $\mathscr{D}_{d}=\mathcal{U}_{L}$.

Demonstração. Inicialmente, observe que $\left\{B^{d}(e, r): r>0\right\}$ é uma base local de $e$ em $(G, \tau)$, uma vez que, por hipótese, $d$ é compatível. E, sendo assim, segue do corolário 3.2.4 que

$$
\mathscr{C}:=\left\{E_{B^{d}(e, r)}: r>0\right\}
$$

é base de $\mathcal{U}_{L}$.

Considere, agora, a coleção $\mathscr{B}_{d}:=\left\{D_{r}^{d}: r>0\right\}$, a qual é, por definição, uma base de $\mathscr{D}_{d}$, e note que, para cada $r>0$, temos que

$$
\begin{aligned}
D_{r}^{d} & =\{(x, y) \in G \times G: d(x, y)<r\}=\left\{(x, y) \in G \times G: d\left(e, x^{-1} y\right)<r\right\} \\
& =\left\{(x, y) \in G \times G: x^{-1} y \in B^{d}(e, r)\right\}=E_{B^{d}(e, r)} .
\end{aligned}
$$

Como $r>0$ é arbitrário, isso mostra que $\mathscr{C}=\mathscr{B}_{d}$. E, sendo $\mathscr{B}_{d}$ e $\mathscr{C}$ bases de $\mathscr{D}_{d}$ e $\mathcal{U}_{L}$, respectivamente, disso concluímos que $\mathscr{D}_{d}=\mathcal{U}_{L}$.

Corolário 3.2.10. A estrutura uniforme à esquerda associada ao grupo aditivo de um espaço normado é a uniformidade induzida pela norma.

\subsection{TOPOLOGIAS INDUZIDAS POR ESTRUTURAS UNIFORMES}

No que segue, mostraremos que uma estrutura uniforme sobre um conjunto não vazio $X$ induz uma topologia sobre $X$ de uma forma bastante natural e, portanto, representa de fato

\footnotetext{
${ }^{4}$ A ideia por trás dessa terminologia é a de que podemos pensar em uma sequência finita $\left(a_{1}, \ldots, a_{k}\right)$ de elementos de $\mathcal{F}$ como sendo uma palavra que representa o elemento $a_{1} \cdot \ldots \cdot a_{k} \in G$. Nessa comparação, cada $a_{i}$ em $\left(a_{1}, \ldots, a_{k}\right)$ corresponderia a uma letra desta palavra, e, por sua, vez, a distância entre dois elementos, $x$ e $y$, de $G$, seria o "tamanho" (isto é, o número de letras) da menor palavra que representa $x^{-1} y$.
} 
mais estrutura sobre $X$ do que uma topologia. Para isso, façamos, primeiramente, uma definição preliminar.

Definição 3.3.1. Seja $X$ um conjunto arbitrário. Para cada $K \subseteq X$ e cada $D \subseteq X \times X$, denotaremos por $D[K]$ o conjunto

$$
\{y \in X: \exists x \in K \text { t.q. }(x, y) \in D\} .
$$

Caso $K=\{x\}$, denotaremos $D[K]$ simplesmente por $D[x]$.

Observe que, pela definição 3.3.1, temos que

$$
D[K]=\bigcup_{x \in K}\{x\}
$$

quaisquer que sejam o conjunto $X$ e os subconjuntos $K \subseteq X$ e $D \subseteq X \times X$.

A proposição a seguir estabelece algumas propriedades simples relacionadas à definição 3.3.1.

Proposição 3.3.2. Seja $X$ um conjunto arbitrário. Para todos $E, F \subseteq X \times X$ e todo $K \subseteq X$, temos que:

a) $(E \circ F)[K]=E[F[K]]$;

b) $(E \cup F)[K]=E[K] \cup F[K]$;

c) $(E \cap F)[K]=E[K] \cap F[K]$;

d) $S e E \subseteq F$, então $E[K] \subseteq F[K]$.

Demonstração. Vamos provar o item a). A demonstração dos demais itens fica a cargo do leitor.

Seja $y \in(E \circ F)[K]$. Por definição, disso segue que existe $x \in K$ tal que $(x, y) \in E \circ F$. Por sua vez,

$$
(x, y) \in E \circ F \Rightarrow \exists z \in X \text { t.q. }(x, z) \in F \text { e }(z, y) \in E .
$$

Note que $z \in F[K]$ (pois $(x, z) \in F$ e $x \in K$ ). Como $(z, y) \in E$, disso concluímos que $y \in E[F[K]]$.

Reciprocamente, dado $y^{\prime} \in E[F[K]]$, temos que existe $z^{\prime} \in F[K]$ tal que $\left(z^{\prime}, y^{\prime}\right) \in E$. Do mesmo modo, como $z^{\prime} \in F[K]$, existe $x^{\prime} \in K$ tal que $\left(x^{\prime}, z^{\prime}\right) \in F$. Observe que

$$
\left[\left(x^{\prime}, z^{\prime}\right) \in F \text { e }\left(z^{\prime}, y^{\prime}\right) \in E\right] \Rightarrow\left(x^{\prime}, y^{\prime}\right) \in E \circ F \text {. }
$$

Como $x^{\prime} \in K$, disso resulta que $y^{\prime} \in(E \circ F)[K]$.

Por fim, como cada elemento de $(E \circ F)[K]$ pertence a $E[F[K]]$, e vice-versa, concluímos que esses dois conjuntos devem ser iguais.

Uma das formas de se definir uma topologia sobre um conjunto $X$ não vazio é especificando uma base local para cada $x \in X$. É isso que faremos a seguir.

TeOrema 3.3.3. Sejam $X$ um conjunto não vazio e $\mathscr{D}$ uma estrutura uniforme sobre $X$. Nessas condições,

$$
\tau:=\{U \subseteq X: \forall x \in U, \exists D \in \mathscr{D} \text { t.q. } D[x] \subseteq U\}
$$

é uma topologia sobre $X$ e, para cada $x \in X, \mathcal{V}_{x}=\{D[x]: D \in \mathscr{D}\}$ é uma base local de $x$ em $(X, \tau)$. 


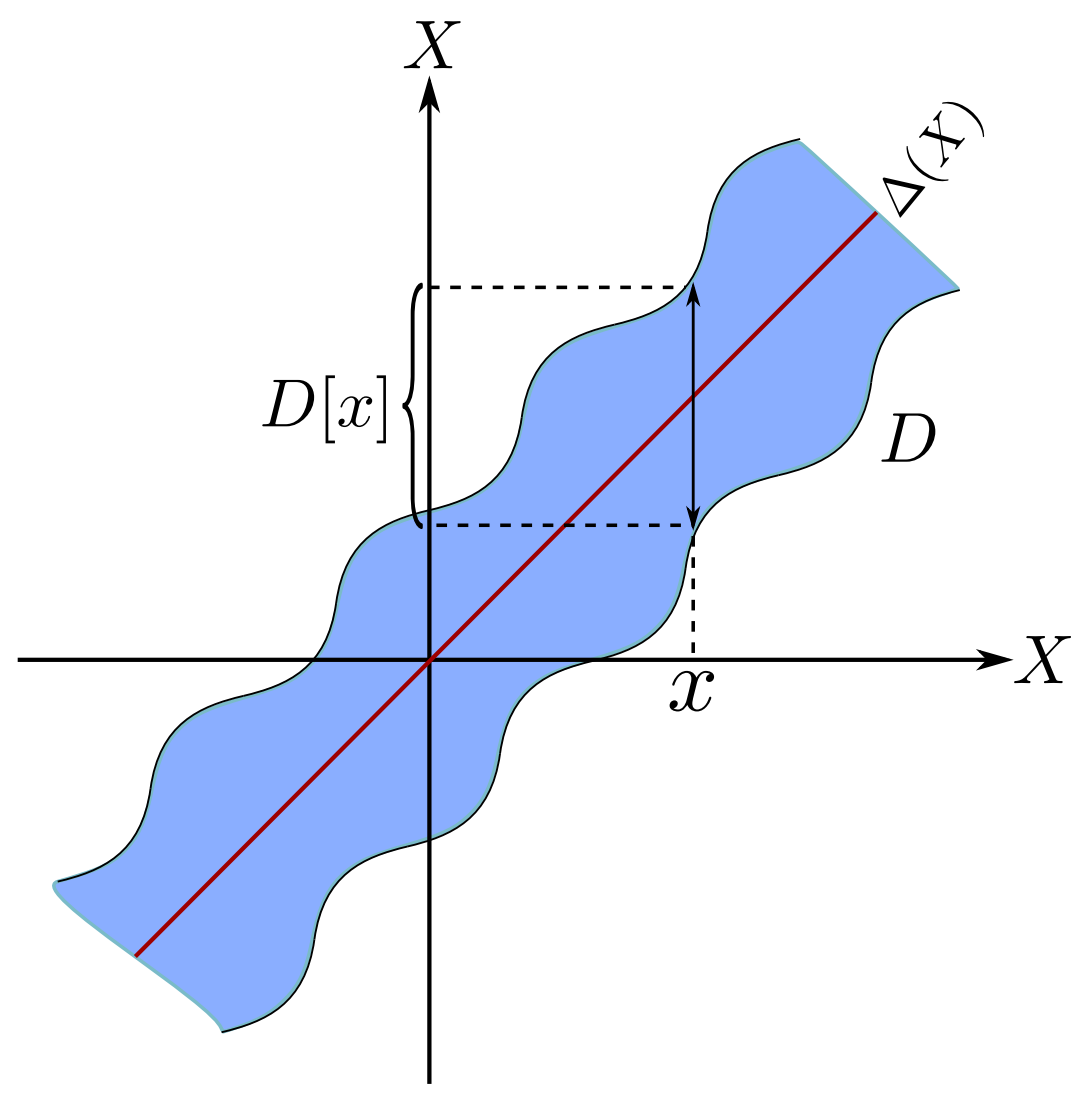

Figura 3.4: Representação de $D[x]$.

Demonstração. Vamos mostrar que, para cada $x \in X, \mathcal{V}_{x}$ satisfaz as propriedades a), b) e c) da proposição 2.7.19, a partir do que seguirá o resultado (pela proposição 2.7.20).

Seja $x \in X$ dado. Inicialmente, note que, para cada $D \in \mathscr{D}$,

$$
(x, x) \in D \Rightarrow x \in D[x] .
$$

Isso mostra que $\mathcal{V}_{x}$ satisfaz a).

A seguir, consideremos $D_{1}, D_{2} \in \mathscr{D}$ quaisquer. Como $D_{1} \cap D_{2} \in \mathscr{D}$ e

$$
\left(D_{1} \cap D_{2}\right)[x]=D_{1}[x] \cap D_{2}[x],
$$

é imediato ver que $D_{1}[x] \cap D_{2}[x] \in \mathcal{V}_{x}$. Disso resulta que $\mathcal{V}_{x}$ satisfaz b).

Por fim, observe que, dado $D \in \mathscr{D}$, segue da definição 3.1.1 que existe $E \in \mathscr{D}$ tal que $E \circ E \subseteq D$. Mostremos que $E[y] \subseteq D[x]$ para todo $y \in E[x]$. De fato, dados $y \in E[x] \mathrm{e}$ $z \in E[y]$, temos que $(x, y),(y, z) \in E$ e, portanto, $(x, z) \in E \circ E \subseteq D$, do que resulta que $z \in D[x]$. Como $y \in E[x]$ e $z \in E[y]$ são arbitrários, isso prova nossa afirmação. E, como $D \in \mathscr{D}$ é arbitrário, disso concluímos que $\mathcal{V}_{x}$ satisfaz c).

OBSERVAÇÃo 3.3.4. Sejam $X, \mathscr{D}$ e $\tau$ como no teorema anterior. Nessas condições, é um exercício simples mostrar que, se $\mathscr{B}$ é uma base de $\mathscr{D}$, então, para cada $x \in X$, a coleção

$$
\mathcal{U}_{x}:=\{B[x]: B \in \mathscr{B}\}
$$


é uma base local de $x$ em $(X, \tau)$. Dessa forma, nada muda se substituírmos $\mathscr{D}$ por qualquer uma de suas bases na definição de $\tau$.

NotAÇÃo: A topologia induzida (ou gerada) por uma uniformidade $\mathscr{D}$ sobre um conjunto $X$ será denotada por $\tau_{\mathscr{D}}$.

É imediato verificar que, se $\mathscr{D}_{1}$ e $\mathscr{D}_{2}$ são estruturas uniformes sobre um mesmo conjunto $X$ tais que $\mathscr{D}_{1}$ é mais fina que $\mathscr{D}_{2}$, então $\tau_{\mathscr{D}_{1}}$ é mais fina que $\tau_{\mathscr{D}_{2}}$.

O teorema 3.3.3 motiva a seguinte definição:

DEFINIÇÃo 3.3.5. Dizemos que um espaço topológico $(X, \tau)$ é uniformizável se existir uma estrutura uniforme $\mathscr{D}$ sobre $X$ tal que $\tau=\tau_{\mathscr{D}}$.

Exemplos 3.3.6. Seja $X$ um conjunto não vazio.

a) A uniformidade discreta induz sobre $X$ a topologia discreta.

b) A uniformidade trivial induz sobre $X$ a topologia caótica.

c) Sejam $d$ uma pseudométrica sobre $X$ e $\mathscr{D}$ a estrutura uniforme induzida por $d$. Mostremos que as topologias induzidas por $\mathscr{D}$ e por $d$ sobre $X$ coincidem.

Como $\mathscr{B}:=\left\{D_{\varepsilon}^{d}: \varepsilon>0\right\}$ é base de $\mathscr{D}$, segue da observação 3.3.4 que

$$
\tau_{\mathscr{D}}=\left\{U \subseteq X: \forall x \in U, \exists \varepsilon>0 \text { t.q. } D_{\varepsilon}^{d}[x] \subseteq U\right\} .
$$

Mas, para cada $x \in X$ e cada $\varepsilon>0$,

$$
D_{\varepsilon}^{d}[x]:=\left\{y \in X:(x, y) \in D_{\varepsilon}^{d}\right\}=\{y \in X: d(x, y)<\varepsilon\}=B_{X}^{d}(x, \varepsilon) .
$$

E, portanto, $\tau_{\mathscr{D}}=\left\{U \subseteq X: \forall x \in U, \exists \varepsilon>0\right.$ t.q. $\left.B_{X}^{d}(x, \varepsilon) \subseteq U\right\}=\tau_{d}$, em que $\tau_{d}$ é a topologia induzida por $d$ sobre $X$.

d) Sejam $A \subseteq X$ um conjunto não vazio, $\mathscr{D}$ uma uniformidade sobre $X$ e

$$
\mathscr{D}_{A}:=\{D \cap(A \times A): D \in \mathscr{D}\}
$$

a uniformidade induzida por $\mathscr{D}$ sobre $A$ (veja o exemplo 3.1.9). Vamos mostrar que $\tau_{\mathscr{D}}=$ $\left(\tau_{\mathscr{D}}\right)_{A}$, em que $\left(\tau_{\mathscr{D}}\right)_{A}$ é a topologia induzida por $\tau_{\mathscr{D}}$ sobre $A$.

Para isso, é suficiente mostrarmos que cada ponto de $A$ admite uma mesma base local em $\left(A, \tau_{\mathscr{D}_{A}}\right)$ e em $\left(A,\left(\tau_{\mathscr{D}}\right)_{A}\right)$.

Seja $x \in A$ um ponto arbitrário. Segue do teorema 3.3.3 que

$$
\mathscr{W}_{x}:=\{[D \cap(A \times A)][x]: D \in \mathscr{D}\}
$$

é uma base local de $x$ em $\left(A, \tau_{\mathscr{D}_{A}}\right)$. Do mesmo modo, temos que $\mathcal{V}_{x}=\{D[x]: D \in \mathscr{D}\}$ é uma base local de $x$ em $\left(X, \tau_{\mathscr{D}}\right)$. Disso resulta que $\mathscr{U}_{x}=\{D[x] \cap A: D \in \mathscr{D}\}$ é uma base local de $x \operatorname{em}\left(A,\left(\tau_{\mathscr{D}}\right)_{A}\right)$.

Por outro lado, note que, para cada $D \in \mathscr{D}$,

$$
[D \cap(A \times A)][x]=D[x] \cap(A \times A)[x]=D[x] \cap A .
$$

Dessa forma, $\mathscr{W}_{x}=\mathscr{U}_{x}$, e o resultado está provado. 
Assim como duas pseudométricas sobre um mesmo conjunto $X$ podem dar origem à mesma uniformidade, será verdade que duas uniformidades sobre $X$ podem induzir a mesma topologia? O próximo exemplo responde (positivamente) a essa questão.

Exemplo 3.3.7. Seja $\mathscr{D}$ a uniformidade sobre $\mathbb{R}$ que tem como base a coleção

$$
\mathscr{B}:=\left\{D_{a}: a \in \mathbb{R}\right\}
$$

em que, para cada $a \in \mathbb{R}$,

$$
D_{a}:=\Delta \cup\left\{(x, y) \in \mathbb{R}^{2}: x>a \text { ou } y>a\right\}
$$

(exemplo 3.1.8-d)). Vamos mostrar que, assim como a uniformidade discreta, $\mathscr{D}$ induz sobre $\mathbb{R}$ a topologia discreta. Para tanto, basta mostrarmos que $\{x\}$ é aberto para cada $x \in \mathbb{R}$. É o que faremos a seguir.

Dado um ponto qualquer $x \in \mathbb{R}$, note que $D_{a}[x]=\{x\}$ para todo $a \geq x$. Como

$$
\left\{D_{a}[x]: a \in \mathbb{R}\right\}
$$

é uma base local de $x$ em $\left(\mathbb{R}, \tau_{\mathscr{D}}\right)$, disso segue, em particular, que $\{x\}$ é uma vizinhança de $x$ em $\left(X, \tau_{\mathscr{D}}\right)$ e, portanto, é aberto (pois é unitário e contém um aberto ao qual $x$ pertence).

Tendo em vista o teorema 3.3.3, faz sentido perguntarmos quais informações podemos inferir sobre uma topologia $\tau_{\mathscr{D}}$, induzida por uma uniformidade $\mathscr{D}$ sobre um conjunto $X$, a partir das propriedades de $\mathscr{D}$; e, reciprocamente, como recuperar informações sobre $\mathscr{D}$, uma vez conhecidas as propriedades de $\tau_{\mathscr{D}}$. Essa é, naturalmente, uma questão bastante geral, uma vez que topologias e uniformidades podem apresentar muitas propriedades de interesse. A seguir, veremos como respondê-la em um caso particular.

Proposição 3.3.8. Sejam $(X, \mathscr{D})$ um espaço uniforme e $\tau$ a topologia induzida por $\mathscr{D}$ sobre $X$. Nessas condições, $(X, \tau)$ será um espaço de Hausdorff se, e somente se, $\bigcap_{D \in \mathscr{D}} D=\Delta$.

Demonstração. $(\Rightarrow)$ Inicialmente, observe que

$$
\Delta \subseteq D \quad \forall D \in \mathscr{D} \Rightarrow \Delta \subseteq \bigcap_{d \in \mathscr{D}} D
$$

Vamos, agora, provar a inclusão oposta. Para isso, tomemos $(x, y) \in(X \times X) \backslash \Delta$ de modo arbitrário. Como $x \neq y$ e $(X, \tau)$ é um espaço de Hausdorff, podemos fixar $D_{1}, D_{2} \in \mathscr{D}$ tais que $D_{1}[x] \cap D_{2}[y]=\emptyset$. Note que

$$
D_{1}[x] \cap D_{2}[y]=\emptyset \Rightarrow y \notin D_{1}[x] \cap D_{2}[y] \underset{y \in D_{2}[y]}{\Longrightarrow} y \notin D_{1}[x] \Rightarrow(x, y) \notin D_{1} \Rightarrow(x, y) \notin \bigcap_{D \in \mathscr{D}} D .
$$

E, sendo $(x, y) \in(X \times X) \backslash \Delta$ um ponto qualquer, disso resulta que $\bigcap_{D \in \mathscr{D}} D \subseteq \Delta$.

$(\Leftarrow)$ Sejam $x, y \in X$ tais que $x \neq y$ dois pontos quaisquer. Observe que, por definição, $(x, y) \notin \Delta$. E, como $\bigcap_{D \in \mathscr{D}} D=\Delta$, disso resulta que existe $D \in \mathscr{D}$ tal que $(x, y) \notin D$. Fixemos, agora, um conjunto simétrico $E \in \mathscr{D}$ tal que $E \circ E \subseteq D$, e vamos mostrar que $E[x] \cap E[y]=\emptyset$. Para isso, basta notar que, para todo $z \in X$,

$$
z \in E[x] \cap E[y] \Rightarrow(x, z),(y, z) \in E \underset{E=E^{-1}}{\Longrightarrow}(x, z),(z, y) \in E \Rightarrow(x, y) \in E \circ E \Rightarrow(x, y) \in D
$$


Como, por hipótese, $(x, y) \notin D$, segue da contrapositiva que $E[x] \cap E[y]=\emptyset$. E, uma vez que $x, y \in X$ tais que $x \neq y$ são completamente arbitrários, disso concluímos que $(X, \tau)$ é um espaço de Hausdorff.

Por fim, mostremos um importante resultado que diz respeito aos grupos topológicos.

Proposição 3.3.9. A topologia $\tau_{L}$ induzida pela estrutura uniforme à esquerda de um grupo topológico $(G, \cdot, \tau)$ coincide com a topologia do grupo.

Demonstração. Dado $x \in G$, sejam

$$
\mathcal{U}_{x}:=\{U \subseteq G: U \text { é vizinhança de } x \text { em }(G, \tau)\}
$$

e

$$
\mathcal{V}_{x}:=\left\{E_{V}[x]: V \in \mathcal{V}\right\}
$$

Vamos mostrar que $\mathcal{U}_{x}=\mathcal{V}_{x}$. Como $\mathcal{U}_{x}$ e $\mathcal{V}_{x}$ são bases locais de $x$ em $(G, \tau)$ e em $\left(G, \tau_{L}\right)$, respectivamente, e $x \in G$ é arbitrário, disso seguirá que $\tau=\tau_{L}$.

Para isso, lembremos que, para cada $g \in G, f_{g}: G \longrightarrow G$ tal que $f_{g}(h)=g h$ é um homeomorfismo em $(G, \tau)$, e $\left(f_{g}\right)^{-1}=f_{g^{-1}}$. Note ainda que, para todo $V \in \mathcal{V}$,

$$
E_{V}[x]:=\left\{y \in G:(x, y) \in E_{V}\right\}=\left\{y \in G: x^{-1} y \in V\right\}=\{y \in G: y \in x V\}=f_{x}(V) .
$$

Seja $V \in \mathcal{V}$. Como $V$ é uma vizinhança de $e, f_{x}(e)=x$ e $f_{x}$ é um homeomorfismo em $(G, \tau)$, segue que $f_{x}(V)$ é uma vizinhança de $x$ em $(G, \tau)$. Mas, por $(*), E_{V}[x]=f_{x}(V)$. Logo: $E_{V}[x] \in \mathcal{U}_{x}$.

Consideremos agora $U \in \mathcal{U}_{x}$ qualquer, e seja $W=f_{x^{-1}}(U)$. Como $U$ é vizinhança de $x$ em $(G, \tau), e=f_{x^{-1}}(x)$ e $f_{x^{-1}}$ é um homeomorfismo em $(G, \tau)$, temos que $f_{x^{-1}}(U)$ é uma vizinhança de $e$ em $(G, \tau)$. Ou seja, $W \in \mathcal{V}$. Por fim, segue de $(*)$ que

$$
E_{W}[x]=f_{x}(W)=f_{x}\left(f_{x^{-1}}(U)\right)=\left(f_{x} \circ f_{x^{-1}}\right)(U)=U
$$

e, portanto, $U \in \mathcal{V}_{x}$.

Como $V \in \mathcal{V}$ e $U \in \mathcal{U}_{x}$ são arbitrários, disso resulta que $\mathcal{U}_{x}=\mathcal{V}_{x}$.

A proposição 3.3.9 tem o seguinte corolário:

Corolário 3.3.10. Seja $(G, \cdot, \tau)$ um grupo topológico. Nessas condições, uma pseudométrica invariante à esquerda d sobre $G$ é compativel (com a topologia do grupo) se, e somente se, $\mathscr{D}_{d}=\mathcal{U}_{L}$.

Demonstração. $(\Rightarrow)$ É a proposição 3.2.9.

$(\Leftarrow)$ Com efeito, sendo $d$ uma pseudométrica sobre $G$ e $(G, \cdot, \tau)$ um grupo topológico, segue de 3.3.6-c) e da proposição anterior que $\tau_{\mathscr{D}_{d}}=\tau_{d}$ e que $\tau=\tau_{\mathcal{U}_{L}}$, a partir do que concluímos que

$$
\tau_{d}=\tau_{\mathscr{D}_{d}}=\tau_{\mathcal{U}_{L}}=\tau
$$

\subsection{FUnÇÕES ENTRE EsPaÇOS UNIFORMES}

Uma das motivações para se definir o conceito de estrutura uniforme é o fato de que ele permite generalizar a noção de continuidade uniforme, definida originalmente para funções entre 
espaços métricos. E isto é, de fato, o que faremos a seguir. Ao longo desta seção, $(X, \mathscr{D})$ e $(Y, \mathscr{F})$ denotarão espaços uniformes quaisquer.

Definição 3.4.1 (Continuidade uniforme). Dizemos que uma função $f: X \longrightarrow Y$ é uniformemente contínua em relação às estruturas uniformes $\mathscr{D}$ e $\mathscr{F}$ se, para cada $F \in \mathscr{F}$, existir $D \in \mathscr{D}$ tal que, para todo $(x, y) \in X \times X$,

$$
(x, y) \in D \Rightarrow(f(x), f(y)) \in F .
$$

Quando as estruturas uniformes estiverem claras pelo contexto, diremos apenas que $f$ é uniformemente contínua.

ObSeRvaÇÃo 3.4.2. Sejam $\mathscr{B}$ uma base de $\mathscr{D}, \mathscr{C}$ uma base de $\mathscr{F}$ e $f: X \longrightarrow Y$ uma função. Note que, para provarmos que $f$ é uniformemente contínua, basta mostrarmos que, para cada $C \in \mathscr{C}$, existe $D \in \mathscr{D}$ tal que $(f(x), f(y)) \in C$ para todo $(x, y) \in D$. Isso decorre imediatamente do fato que cada $F \in \mathcal{F}$ contém um elemento de $\mathscr{C}$. Além disso, como cada $D \in \mathscr{D}$ contém um elemento de $\mathscr{B}$, é fácil ver que, se $f$ for uniformemente contínua, então, para cada $F \in \mathscr{F}$, existe $B \in \mathscr{B}$ tal que $(f(x), f(y)) \in F$ para todo $(x, y) \in B$.

Como vimos, uma estrutura uniforme induz uma topologia sobre o seu conjunto base. Isso nos permite transportar conceitos topológicos para espaços uniformes de forma bastante natural. Um exemplo particularmente importante é a continuidade.

Definição 3.4.3 (Continuidade). Dizemos que uma função $f: X \longrightarrow Y$ é contínua em relação às unifomidades $\mathscr{D}$ e $\mathscr{F}$ se for contínua em relação às topologias induzidas $\tau_{\mathscr{D}}$ e $\tau_{\mathscr{F}}$.

O próximo resultado relaciona continuidade e continuidade uniforme para funções entre $(X, \mathscr{D})$ e $(Y, \mathscr{F})$.

Proposição 3.4.4. Toda função uniformemente contínua é contínua.

Demonstração. Dada $f: X \longrightarrow Y$ uniformemente contínua, tomemos $x \in X$ e $V \in \tau_{\mathscr{F}}$ tais que $f(x) \in V$. Pela definição de $\tau_{\mathscr{F}}$, existe $F \in \mathscr{F}$ tal que $F[f(x)] \subseteq V$. Por outro lado, como $f$ é uniformemente contínua, existe $D \in \mathscr{D}$ tal que $(f(x), f(y)) \in F$ para cada $(x, y) \in D$. Observe que $f(D[x]) \subseteq F[f(x)] \subseteq V$. Como $x \in X$ e $V \in \tau_{\mathscr{F}}$ tal que $f(x) \in V$ são arbitrários, disso concluímos que $f$ é contínua.

EXEMPLOS 3.4.5.

a) Se $\mathscr{F}$ for a uniformidade trivial, toda função $f: X \longrightarrow Y$ será uniformemente contínua.

b) Se $\mathscr{D}$ for a uniformidade discreta, toda função $f: X \longrightarrow Y$ será uniformemente contínua. Isso decorre do fato de que, para todo $F \in \mathscr{F}$ e todo $(x, y) \in X \times X$ :

$$
(x, y) \in \Delta(X) \Rightarrow x=y \Rightarrow f(x)=f(y) \Rightarrow(f(x), f(y)) \in \Delta(Y) \subseteq F .
$$

c) Se $Z \subseteq X$ for um subconjunto não vazio e $f: X \longrightarrow Y$ for uma função uniformemente contínua em relação a $\mathscr{D}$ e $\mathscr{F}$, a restrição de $f$ a $Z$ será uniformemente contínua em relação a $\mathscr{D}_{Z}$ e $\mathscr{F}$.

Demonstração. Seja $F \in \mathscr{F}$ dado. Como $f$ é uniformemente contínua, podemos fixar $D \in \mathscr{D}$ de modo que $(f(x), f(y)) \in F$ para todo $(x, y) \in D$. Tomemos $D^{\prime}=D \cap(Z \times Z)$. Note que $D^{\prime} \in \mathscr{D}_{Z}$. Além disso, para todo $(x, y) \in X \times X$,

$$
(x, y) \in D^{\prime} \Rightarrow(x, y) \in D \Rightarrow(f(x), f(y)) \in F \Rightarrow\left(f 1_{Z}(x), f 1_{Z}(y)\right) \in F .
$$


Como $F \in \mathscr{F}$ é arbitrário, disso segue que $f 1_{Z}$ é uniformemente contínua.

d) Analisemos o caso em que $\mathscr{D}$ e $\mathscr{F}$ são induzidas por métricas (ou pseudométricas) $d$ e $\partial$, respectivamente. Veremos que, nesse caso, a definição de continuidade uniforme é equivalente à definição usual para funções entre espaços métricos.

Para isso, definamos $\mathscr{B}:=\left\{D_{\delta}^{d}: \delta>0\right\}$ e $\mathscr{C}:=\left\{F_{\varepsilon}^{\partial}: \varepsilon>0\right\}$, e consideremos uma função $f$ de $X$ em $Y$. Vamos mostrar que $f$ é uniformemente contínua (no sentido da definição 3.4.1) se, e somente se, para todo $\varepsilon>0$, existe um $\delta>0$ tal que $\partial(f(x), f(y))<\varepsilon$ para todos $x, y \in X$ tais que $d(x, y)<\delta$.

$(\Rightarrow)$ Seja $\varepsilon>0$ dado. Como $f: X \longrightarrow Y$ é uniformemente contínua e $\mathscr{B}$ é base de $\mathscr{D}$, segue da observação 3.4 .2 que existe $\delta>0$ tal que $(f(x), f(y)) \in F_{\varepsilon}^{\partial}$ para todo $(x, y) \in D_{\delta}^{d}$. Logo, para todos $x, y \in X$,

$$
d(x, y)<\delta \Rightarrow(x, y) \in D_{\delta}^{d} \Rightarrow(f(x), f(y)) \in F_{\varepsilon}^{\partial} \Rightarrow \partial(f(x), f(y))<\varepsilon .
$$

$(\Leftarrow)$ Seja $F \in \mathscr{F}$ dado. Como $\mathscr{C}$ é base de $\mathscr{F}$, podemos fixar $\varepsilon>0$ tal que $F_{\varepsilon}^{\partial} \subseteq F$. Por hipótese, existe $\delta>0$ tal que $\partial(f(x), f(y))<\varepsilon$ para todos $x, y \in X$ tais que $d(x, y)<\delta$. Sendo assim, para todo $(x, y) \in X \times X$,

$$
(x, y) \in D_{\delta}^{d} \Rightarrow d(x, y)<\delta \Rightarrow \partial(f(x), f(y))<\varepsilon \Rightarrow(f(x), f(y)) \in F_{\varepsilon}^{\partial} \Rightarrow(f(x), f(y)) \in F .
$$

Como $F \in \mathscr{F}$ é arbitrário, disso resulta que $f$ é uniformemente contínua.

A seguir, provaremos duas propriedades simples das funções uniformemente contínuas, as quais decorrem facilmente da definição 3.4.1. Antes disso, porém, vamos introduzir uma notação conveniente, que será utilizada ao longo de todo o trabalho.

DEFINIÇÃo 3.4.6. Dados subconjuntos $E \subseteq X \times X$ e $F \subseteq Y \times Y$, e uma função $f: X \longrightarrow Y$, denotaremos por $F_{f}$ o conjunto

$$
\{(x, y) \in X \times X:(f(x), f(y)) \in F\}
$$

e por $E^{f}$ o conjunto

$$
\{(f(x), f(y)):(x, y) \in E\}
$$

Proposição 3.4.7. Uma função $f: X \longrightarrow Y$ é uniformemente contínua se, e somente se, $F_{f} \in \mathscr{D}$ para cada $F \in \mathscr{F}$.

Demonstração. $(\Rightarrow)$ Seja $F \in \mathscr{F}$ dado. Como $f$ é uniformemente contínua, podemos fixar $D \in \mathscr{D}$ de modo que $(f(x), f(y)) \in F$ para todo $(x, y) \in D$. Disso segue que, para todo $(x, y) \in X \times X$

$$
(x, y) \in D \Rightarrow(f(x), f(y)) \in F \Rightarrow(x, y) \in F_{f},
$$

a partir do que concluímos que $D \subseteq F_{f}$.

Por fim, como $D \in \mathscr{D}$ e $D \subseteq F_{f}$, concluímos que $F_{f} \in \mathscr{D}$.

$(\Leftarrow)$ Note que, dado $F \in \mathscr{F}$, temos, por hipótese, que $F_{f} \in \mathscr{D}$. Além disso, segue da definição de $F_{f}$ que $(f(x), f(y)) \in F$ para todo $(x, y) \in F_{f}$. E, como $F \in \mathscr{F}$ é arbitrário, disso resulta que $f$ é uniformemente contínua.

Observe que a proposição 3.4.7 nos fornece uma definição alternativa para a continuidade uniforme. 
Proposição 3.4.8. A composição de funções uniformemente continuas é uniformemente contínu.

Demonstração. Dados espaços uniformes $(X, \mathscr{D}),(Y, \mathscr{F})$ e $(Z, \mathscr{G})$, e funções uniformemente contínuas $f: X \longrightarrow Y$ e $g: Y \longrightarrow Z$, vamos mostrar que $g \circ f: X \longrightarrow Z$ é uniformemente contínua.

Seja $G \in \mathscr{G}$ dado. Como $g$ é uniformemente contínua, podemos tomar $F \in \mathscr{F}$ de modo que $(g(u), g(v)) \in G$ para todo $(u, v) \in F$. Por sua vez, como $f$ é uniformemente contínua, podemos, agora, fixar $D \in \mathscr{D}$ tal que $(f(x), f(y)) \in F$ para todo $(x, y) \in D$. Logo, para todo $(x, y) \in X \times X$

$$
(x, y) \in D \Rightarrow(f(x), f(y)) \in F \Rightarrow(g(f(x)), g(f(y))) \in G \Rightarrow((g \circ f)(x),(g \circ f)(y)) \in G .
$$

Como $G \in \mathscr{G}$ é arbitrário, disso segue que $g \circ f$ é uniformemente contínua.

Definição 3.4.9 (Mergulho uniforme). Dizemos que uma função $f: X \longrightarrow Y$ é um mergulho uniforme em relação às estruturas uniformes $\mathscr{D}$ e $\mathscr{F}$ se for uniformemente contínua em relação a $\mathscr{D}$ e $\mathscr{F}$ e, para cada $D \in \mathscr{D}$, existir $F \in \mathscr{F}$ tal que, para todo $(x, y) \in X \times X$,

$$
(x, y) \notin D \Rightarrow(f(x), f(y)) \notin F .
$$

Também neste caso, quando as estruturas uniformes estiverem claras pelo contexto, diremos apenas que $f$ é um mergulho uniforme.

OBSERVAÇÃo 3.4.10. Considerações análogas às da observação 3.4.2 também podem ser feitas no caso dos mergulhos uniformes. Com efeito, dadas bases $\mathscr{B}$ e $\mathscr{C}$ de $\mathscr{D}$ e de $\mathscr{F}$, respectivamente, e uma função uniformemente contínua $f: X \longrightarrow Y$, são equivalentes:

a) $f$ é um mergulho uniforme;

b) Para todo $B \in \mathscr{B}$, existe $F \in \mathscr{F}$ tal que $(f(x), f(y)) \notin F$ para todos $x, y \in X$ tais que $(x, y) \notin B$

c) Para todo $D \in \mathscr{D}$, existe $C \in \mathscr{C}$ tal que $(f(x), f(y)) \notin C$ para todos $x, y \in X$ tais que $(x, y) \notin D$.

EXEMPLOS 3.4.11.

a) Se $\mathscr{D}$ e $\mathscr{F}$ forem ambas as respectivas uniformidades discretas, toda função injetora $f$ de $X$ em $Y$ será um mergulho uniforme.

b) Caso $\mathscr{D}$ e $\mathscr{F}$ sejam induzidas por pseudométricas $d$ e $\partial$, respectivamente, pode-se mostrar que uma função uniformemente contínua $f: X \longrightarrow Y$ será um mergulho uniforme se, e somente se, para todo $\Delta>0$, existir $M>0$ tal que $\partial(f(x), f(y)) \geq M$ para todos $x, y \in X$ tais que $d(x, y) \geq \Delta$. A demonstração disso segue facilmente da observação 3.4 .10 e é análoga à que foi feita no exemplo 3.4.5-d).

Proposição 3.4.12. A composição de mergulhos uniformes é um mergulho uniforme.

Demonstração. Fica a cargo do leitor. 
Note que, se $Z \subseteq X$ é não vazio e $f: Z \longrightarrow Y$ é uma função, então $f$ será uniformemente contínua (em relação a $\mathscr{D}_{Z}$ e $\mathscr{F}$ ) se, e somente se, para cada $F \in \mathscr{F}$, existir $D \in \mathscr{D}$ tal que $(f(x), f(y)) \in F$ para todo $(x, y) \in D \cap(Z \times Z)$. Analogamente, $f$ será um mergulho uniforme se, e somente se, for uniformemente contínua e, para cada $D \in \mathscr{D}$, existir $F \in \mathscr{F}$ tal que, para todo $(x, y) \in Z \times Z$,

$$
(x, y) \notin D \Rightarrow(f(x), f(y)) \notin F .
$$

A proposição a seguir estabelece uma caracterização para os mergulhos uniformes injetores em termos da continuidade uniforme.

Proposição 3.4.13. Uma função injetora $f: X \longrightarrow Y$ é um mergulho uniforme se, $e$ somente se, $f e$

$$
f^{-1}: \operatorname{Im}(f) \longrightarrow X
$$

são uniformemente contínuas.

Demonstração. $(\Rightarrow)$ A continuidade uniforme de $f$ decorre diretamente da definição de mergulho uniforme. Além disso, como $f$ é injetora, temos que $f^{-1}: \operatorname{Im}(f) \longrightarrow X$ está bem definida. Mostremos que $f^{-1}$ é uniformemente contínua.

Seja $D \in \mathscr{D}$ dado. Como $f$ é um mergulho uniforme, podemos tomar $F \in \mathscr{F}$ de modo que $(f(x), f(y)) \notin F$ para todos $x, y \in X$ tais que $(x, y) \notin D$. Pela contrapositiva, disso segue que, se $(f(x), f(y))$ pertence a $F$, então $(x, y) \in D$. Logo, para todos $a, b \in \operatorname{Im}(f)$,

$$
(a, b) \in F \Rightarrow\left(f\left(f^{-1}(a)\right), f\left(f^{-1}(b)\right)\right) \in F \Rightarrow\left(f^{-1}(a), f^{-1}(b)\right) \in D .
$$

Como $D \in \mathscr{D}$ é arbitrário, disso resulta que $f^{-1}$ é uniformemente contínua.

$(\Leftarrow)$ Para concluírmos que $f$ é um mergulho uniforme, precisamos mostrar que, para cada $D \in \mathscr{D}$, existe $F \in \mathscr{F}$ tal que $(f(x), f(y)) \notin F$ para todos $x, y \in X$ tais que $(x, y) \notin D$.

Para isso, tomemos $D \in \mathscr{D}$ qualquer. Como $f^{-1}$ é uniformemente contínua, podemos fixar $F \in \mathscr{F}$ de modo que $\left(f^{-1}(a), f^{-1}(b)\right) \in D$ para todo $(a, b) \in F \cap[\operatorname{Im}(f) \times \operatorname{Im}(f)]$. Disso resulta que, se $a, b \in \operatorname{Im}(f)$ são tais que $\left(f^{-1}(a), f^{-1}(b)\right) \notin D$, então $(a, b) \notin F$. Dessa forma, para todo $(x, y) \in X \times X$,

$$
(x, y) \notin D \Rightarrow\left(f^{-1}(f(x)), f^{-1}(f(y))\right) \notin D \Rightarrow(f(x), f(y)) \notin F .
$$

A partir da proposição 3.4.13, podemos derivar um interessante corolário a respeito de mergulhos uniformes entre espaços métricos. Vamos mostrar que, nesse caso, a injetividade já está automaticamente assegurada.

CorolÁrio 3.4.14. Sejam $(X, d)$ e $(Y, \partial)$ espaços métricos. Nessas condições, uma função $f: X \longrightarrow Y$ será um mergulho uniforme (em relação às uniformidades induzidas pelas métricas) se, e somente se, for injetora e tanto $f$ quanto $f^{-1}: \operatorname{Im}(f) \longrightarrow X$ forem uniformemente contínuas.

Demonstração. $(\Rightarrow)$ Mostremos que $f$ é, necessariamente, injetora. A partir disso e da proposição 3.4.13, seguirá que $f$ e $f^{-1}$ são uniformemente contínuas.

Sejam $x, y \in X$ tais que $x \neq y$. Como $d$ é uma métrica sobre $X$, disso segue que $d(x, y)>0$. Tomemos $\Delta:=d(x, y)$. Como $f$ é um mergulho uniforme, podemos fixar $M>0$ tal que $\partial(f(z), f(w)) \geq M$ para todos $z, w \in X$ tais que $d(z, w) \geq \Delta$. Em particular, disso resulta que $\partial(f(x), f(y)) \geq M>0$ e, portanto, $f(x) \neq f(y)$.

$(\Leftarrow)$ Segue diretamente da proposição 3.4.13. 
O próximo exemplo mostra que a afirmação de que todo mergulho uniforme é injetor, que vale no caso de mergulhos uniformes entre espaços métricos, é falsa em geral.

Exemplo 3.4.15. Se $\mathscr{D}=\{X \times X\}$ e $\mathscr{F}=\{Y \times Y\}$, toda função $f: X \longrightarrow Y$ é um mergulho uniforme.

Em uma primeira comparação, poderíamos dizer que as funções uniformemente contínuas estão para os espaços uniformes assim como as funções contínuas estão para os espaços topológicos. Levando este paralelo adiante, poderíamos tentar estabelecer o análogo, no contexto dos espaços uniformes, aos homeomorfismos entre espaços topológicos. Uma vez que um homeomorfismo consiste em uma função contínua, bijetora e com inversa contínua, fica claro que, nesse caso, o candidato natural a "homeomorfismo uniforme" é uma função uniformemente contínua, bijetora, e com inversa uniformemente contínua. Isso motiva as seguintes definições:

\section{DEFINIÇÕES 3.4.16 (ISOMORFISMO UNIFORME).}

a) Uma função $f: X \longrightarrow Y$ é dita um isomorfismo uniforme (ou uma equivalência uniforme) em relação a $\mathscr{D}$ e $\mathscr{F}$ se for bijetora e tanto $f$ quanto $f^{-1}$ forem uniformemente contínuas.

(Observe que um isomorfismo uniforme nada mais é do que um mergulho uniforme bijetor.)

b) Dizemos que $(X, \mathscr{D})$ e $(Y, \mathscr{F})$ são uniformemente isomorfos (ou uniformemente equivalentes) se existir um isomorfismo uniforme $f: X \longrightarrow Y$ em relação às uniformidades $\mathscr{D}$ e $\mathscr{F}$.

\section{OBSERVAÇÃO 3.4.17.}

a) Note que id $: X \longrightarrow X$ tal que id $(x)=x$ é um isomorfismo uniforme em relação a qualquer estrutura uniforme sobre $X$. Disso resulta que qualquer espaço uniforme é uniformemente isomorfo a si mesmo.

b) É fácil ver que a inversa de um isomorfismo uniforme também é um isomorfismo uniforme. Além disso, segue da proposição 3.4 .8 que a composição de isomorfismos uniformes é um isomorfismo uniforme.

c) Segue da proposição 3.4.4 que, se $f: X \longrightarrow Y$ é um isomorfismo uniforme entre os espaços uniformes $(X, \mathscr{D})$ e $(Y, \mathscr{F})$, então $f$ é um homeomorfismo entre $\left(X, \tau_{\mathscr{D}}\right)$ e $\left(Y, \tau_{\mathscr{F}}\right)$. Dessa forma, espaços uniformemente equivalentes são homeomorfos como espaços topológicos (em relação às topologias induzidas pelas respectivas uniformidades). Note que isso está de acordo com o esperado com base no paralelo anteriormente traçado entre os espaços uniformes e os espaços topológicos.

Seja $\sim$ a relação definida sobre a classe de todos os pares de espaços uniformes de modo que $(X, \mathscr{D}) \sim(Y, \mathscr{F})$ se, e somente se, $(X, \mathscr{D})$ e $(Y, \mathscr{F})$ são uniformemente isomorfos. Segue facilmente dos itens a) e b) da observação 3.4.17 que é uma relação de equivalência. Isso justifica o nome equivalência uniforme, pelo qual também são conhecidos os isomorfismos uniformes.

Em virtude do paralelo entre espaços uniformes e espaços topológicos, também é comum nos referirmos aos isomorfismos uniformes como homeomorfismos uniformes. Nessa terminologia, dois espaços uniformemente isomorfos são ditos uniformemente homeomorfos.

O próximo resultado, o qual diz respeito aos isomorfismos uniformes, é uma consequência direta da proposição 3.4.7.

Proposição 3.4.18. Seja $f: X \longrightarrow Y$ um isomorfismo uniforme. Nessas condições, $D^{f}$ pertence a $\mathscr{F}$ para todo $D \in \mathscr{D}$. 
Demonstração. Seja $D \in \mathscr{D}$ dado. Como $f$ é um isomorfismo uniforme, temos, em particular, que $f$ é bijetora e que $f^{-1}: Y \longrightarrow X$ é uniformemente contínua. Disso segue que

$$
D^{f}:=\{(f(x), f(y)):(x, y) \in D\}=\left\{(a, b) \in Y \times Y:\left(f^{-1}(a), f^{-1}(b)\right) \in D\right\} .
$$

E, assim sendo, podemos aplicar a proposição 3.4 .7 a $f^{-1}$ para concluir que $D^{f} \in \mathscr{F}$.

Encerraremos, por hora, a nossa discussão sobre espaços uniformes com algumas considerações a respeito dos mergulhos uniformes injetores.

OBSERVAÇÃo 3.4.19. Se $f: X \longrightarrow Y$ for uniformemente contínua em relação a $\mathscr{D}$ e $\mathscr{F}$, então $f^{\prime}: X \longrightarrow \operatorname{Im}(f)$ tal que $f^{\prime}(x)=f(x)$ será uniformemente contínua em relação a $\mathscr{D}$ e $\mathscr{F} \operatorname{Im}(f)$. Do mesmo modo, se $f$ for um mergulho uniforme, $f^{\prime}$ será, também, um mergulho uniforme. Com efeito, isso segue diretamente do fato de que, para todo $F \in \mathscr{F}$ e todo $(x, y) \in X \times X$,

$$
(f(x), f(y)) \in F \Leftrightarrow(f(x), f(y)) \in F \cap(\operatorname{Im}(f) \times \operatorname{Im}(f)) .
$$

A partir da proposição 3.4.13 e da observação 3.4.19, podemos deduzir, imediatamente, o próximo corolário.

Corolário 3.4.20. Se $f: X \longrightarrow Y$ for um mergulho uniforme injetor, a função

$$
\begin{aligned}
f^{\prime}: X & \longrightarrow \operatorname{Im}(f) \\
x & \longmapsto f(x)
\end{aligned}
$$

será uma equivalência uniforme (em relação a $\mathscr{D}$ e $\left.\mathscr{F}_{\operatorname{Im}(f)}\right)$.

(E, portanto, $(X, \mathscr{D})$ será uniformemente isomorfo ao subespaço uniforme $\left(\operatorname{Im}(f), \mathscr{F}_{\operatorname{Im}(f)}\right)$ de $(Y, \mathscr{F})$.

O corolário 3.4.20 nos mostra que, se $f: X \longrightarrow Y$ for um mergulho uniforme injetor, podemos enxergar o subespaço $(\operatorname{Im}(f), \mathscr{F} \operatorname{Im}(f))$ de $(Y, \mathscr{F})$ como uma "cópia" de $(X, \mathscr{D})$ dentro de $(Y, \mathscr{F})$. Tendo isso em mente, muitas vezes mostra-se conveniente considerar o próprio $(X, \mathscr{D})$ como subespaço de $(Y, \mathscr{F})$. Isso está de acordo com a ideia intuitiva que temos a respeito de "mergulhar um espaço em outro" e justifica o uso do termo "mergulho uniforme".

Contudo, alguém poderia observar que, uma vez que o parágrafo anterior diz respeito a funções injetoras, o uso do termo "mergulho uniforme" seria adequado para se referir apenas aos mergulhos uniformes injetores. Essa pessoa não deixaria de ter uma certa razão. O que ocorre é que o termo "mergulho uniforme" surgiu originalmente ao se estudar funções entre espaços métricos e, como vimos no corolário 3.4.14, no caso em que as uniformidades $\mathscr{D}$ e $\mathscr{F}$ são induzidas por métricas, todo mergulho uniforme é, automaticamente, injetor. Dessa forma, podemos considerar a definição 3.4.9 como uma generalização do conceito de mergulho uniforme entre espaços métricos para o contexto mais geral dos espaços uniformes. 


\section{Capítulo 4}

\section{ESPAÇOS COARSE}

Quando definimos continuidade ou continuidade uniforme para uma função entre espaços métricos, deixamos de lado uma grande parte da informação carregada pelas respectivas métricas. Como todo estudante de Cálculo sabe, continuidade é uma propriedade local! Isto é, para determinar se uma dada função é contínua em um certo ponto de seu domínio, tudo que importa é o comportamento da função em uma vizinhança (possivelmente, pequena) do ponto em questão. Com efeito, dada uma métrica $d$ sobre um conjunto não vazio $X$, não é difícil mostrar que $d^{\prime}: X \times X \longrightarrow \mathbb{R}$ tal que $d^{\prime}(x, y)=\min \{1, d(x, y)\}$ é também uma métrica sobre $X$, e que as uniformidades (e, portanto, também as topologias) induzidas sobre $X$ por $d$ e por $d^{\prime}$ são as mesmas. E, no entanto, é evidente que, ao substituírmos $d$ por $d^{\prime}$, abrimos mão de toda a informação a respeito das $d$-distâncias maiores que 1.

Neste capítulo, vamos lidar com uma situação, de certa forma, dual em relação ao que foi discutido acima. Ao invés de focarmos nossa atenção na estrutura de pequena escala associada a uma uniformidade, estaremos interessados em estruturas de larga escala. Para isso, vamos precisar dos conceitos de estrutura coarse e espaço coarse, introduzidos por John Roe em 2003 [Roe03]. Uma vez munidos do ferramental matemático introduzido pela teoria dos espaços coarse, seremos capazes de atribuir um sentido preciso a afirmações como " $\mathbb{R}$ e $\mathbb{Z}$ são os mesmos de um ponto de vista de larga escala"; ou então "de uma perspectiva de larga escala, um conjunto limitado é equivalente a um ponto".

As primeiras duas seções destinam-se a familiarizar o leitor com o conceito de estrutura coarse. Por essa razão, vamos, inicialmente, apresentar algumas das definições relevantes e, em seguida, ilustrá-las por meio de exemplos simples. ${ }^{1}$ Uma vez que o leitor esteja razoalvelmente confortável com as definições básicas, desviaremos nossa atenção para a classe dos grupos topológicos. Mais especificamente, vamos motivar e introduzir a estrutura coarse à esquerda de um grupo topológico. ${ }^{2}$ E, por fim, direcionaremos o foco para o estudo de funções entre espaços coarse. Nessa útima seção, mostraremos como redefinir conceitos como bornologia, expansividade, equivalência grosseira e mergulho grosseiro, originalmente associados a funções entre espaços métricos, no contexto mais geral dos espaços coarse.

\footnotetext{
${ }^{1}$ Ao longo da leitura, observe a semelhança de abordagem entre as seções iniciais deste capítulo e do capítulo anterior, a qual, mais do que um mero reflexo de certas escolhas particulares do autor, é consequência de uma grande similaridade operacional entre as estruturas uniformes e as estruturas coarse.

${ }^{2}$ Assim chamada em uma clara alusão à estrutura uniforme à esquerda de um grupo topológico.
} 


\subsection{Estruturas COARSE}

Tendo já os comentários introdutórios acerca da teoria dos espaços coarse sido feitos na introdução do capítulo, nada melhor do que iniciar esta seção com a definição de estrutura coarse.

Definição 4.1.1 (Estrutura COARSE). Seja $X$ um conjunto não vazio. Uma estrutura coarse sobre $X$ é uma coleção $\mathcal{E}$ de subconjuntos de $X \times X$, chamados conjuntos controlados, com as seguintes propriedades:

a) $\Delta \in \mathcal{E}$

b) Se $E, F \in \mathcal{E}$, então $E \cup F \in \mathcal{E}$;

c) Se $E \in \mathcal{E}$, então $E^{-1} \in \mathcal{E}$;

d) Se $E, F \in \mathcal{E}$, então $E \circ F \in \mathcal{E}$;

e) Se $E \in \mathcal{E}$ e $F \subseteq E$, então $F \in \mathcal{E}$.

Observe que uma estrutura coarse $\mathcal{E}$ sobre um conjunto não vazio $X$ é um ideal sobre $X \times X$. Isso decore diretamente das propriedades b) e e) da definição 4.1 .1 e do fato de que $\mathcal{E}$ é não vazio (pois $\Delta \in \mathcal{E}$ ).

A intuição por trás da definição 4.1.1 é a de que os conjuntos controlados de uma estrutura coarse possuem "tamanho negligenciável". Por essa razão, dizemos também que um elemento $E$ de uma estrutura coarse $\mathcal{E}$ é $\mathcal{E}$-nulo ou $\mathcal{E}$-negligenciável. Essa noção tem sua origem no caso particular das estruturas coarse induzidas por pseudométricas, e ficará mais clara uma vez que tenhamos estudado o exemplo 4.1.11. Apesar disso, uma olhada prévia na figura 4.1 pode ajudar o leitor a perceber melhor as ideias presentes na definição.

OBSERVAÇÃo 4.1.2. Note que podemos substituir a propriedade d) da definição 4.1.1 por

d') Se $E \in \mathcal{E}$, então $E \circ E \in \mathcal{E}$.

De fato, é imediato ver que d') é um caso particular de d). Mostremos agora que, se $X$ é um conjunto não vazio e $\mathcal{E}$ é uma coleção de subconjuntos de $X \times X$ com as propriedades a), b), c), d') e e), então $\mathcal{E}$ também satisfaz d).

Com efeito, dados $E, F \in \mathcal{E}$, segue de b) que $E \cup F \in \mathcal{E}$. Mas, por d'):

$$
E \cup F \in \mathcal{E} \Rightarrow(E \cup F) \circ(E \cup F) \in \mathcal{E}
$$

E, por e):

$$
(E \circ F \subseteq(E \cup F) \circ(E \cup F) \text { e }(E \cup F) \circ(E \cup F) \in \mathcal{E}) \Rightarrow E \circ F \in \mathcal{E} .
$$

Logo: $E \circ F \in \mathcal{E}$.

DefiniÇão 4.1.3. Dadas estruturas coarse $\mathcal{E}$ e $\mathcal{F}$ sobre um mesmo conjunto $X$, dizemos que $\mathcal{E}$ é mais fina que $\mathcal{F}$ se $\mathcal{E} \subseteq \mathcal{F}$. Note que isso é o oposto do que ocorre com estruturas uniformes.

O conceito de estrutura coarse motiva a seguinte definição:

DeFiniçÃo 4.1.4 (EsPaÇo COARSE). Um espaço coarse é um par $(X, \mathcal{E})$, em que $X$ é um conjunto não vazio e $\mathcal{E}$ é uma estrutura coarse sobre $X$. 
Seja $\mathcal{E}$ uma estrutura coarse sobre um conjunto $X$. À semelhança do que ocorre no caso das estruturas uniformes, um rápido exame da propriedade e) da definição 4.1.1 pode nos dar a impressão de que $\mathcal{E}$ possui "conjuntos demais", isto é, de que podemos estudar suas propriedades a partir de uma subcoleção (de preferência, menor) de $\mathcal{E}$. Isso nos motiva a definir o conceito de base de uma estrutura coarse, que nada mais é do que uma adaptação da definição 3.1 .4 ao contexto das estruturas coarse.

Definição 4.1.5 (BASE). Sejam $X$ um conjunto não vazio e $\mathcal{E}$ uma estrutura coarse sobre $X$. Dizemos que uma subcoleção $\mathscr{B} \subseteq \mathcal{E}$ é uma base de $\mathcal{E}$ se cada $E \in \mathcal{E}$ estiver contido em algum elemento de $\mathscr{B}$.

Do ponto de vista operacional, a forma de se trabalhar com bases de uma estrutura uniforme e de uma estrutura coarse é, de modo geral, muito parecida. Em razão dessa semelhança, muitas das observações e resultados válidos para bases de estruturas uniformes podem ser prontamente adaptados para bases de estruturas coarse. A seguir, enunciaremos alguns deles.

OBSERVAÇÕES 4.1.6.

a) Toda estrutura coarse é uma base de si mesma. Em particular, disso segue que toda estrutura coarse admite, ao menos, uma base.

b) Dadas uma estrutura coarse $\mathcal{E}$ sobre um conjunto $X$ e uma base $\mathscr{B}$ de $\mathcal{E}$, decorre diretamente da definição 4.1.5 e da propriedade e) da definição 4.1.1 que

$$
\mathcal{E}=\{E \subseteq X \times X: \exists B \in \mathscr{B} \text { t.q. } E \subseteq B\}
$$

Ao longo do texto, muitas vezes será conveniente descrever as estruturas coarse por meio de suas bases. Nesses casos, a próxima proposição pode nos ajudar a estabelecer relações de inclusão entre estruturas coarse. Sua demonstração é simples, e decorre imediatamente do item b) da observação 4.1.6.

Proposição 4.1.7. Sejam $\mathcal{E}_{1}$ e $\mathcal{E}_{2}$ estruturas coarse sobre um mesmo conjunto $X$. Nessas condições, $\mathcal{E}_{1} \subseteq \mathcal{E}_{2}$ se, e somente se, existirem bases $\mathscr{B}_{1}$ e $\mathscr{B}_{2}$ de $\mathcal{E}_{1}$ e de $\mathcal{E}_{2}$, respectivamente, tais que cada elemento de $\mathscr{B}_{1}$ esteja contido em um elemento de $\mathscr{B}_{2}$.

Assim como no caso das estruturas uniformes, podemos estabelecer condições necessárias e suficientes para que uma coleção $\mathscr{B} \subseteq \mathcal{P}(X \times X)$ seja base de uma estrutura coarse sobre $X$. Tendo em vista o item b) da observação 4.1 .6 e a definição 4.1.5, é fácil ver que isso ocorrerá se, e somente se, a coleção

$$
\mathcal{E}:=\{E \subseteq X \times X: \exists B \in \mathscr{B} \text { t.q. } E \subseteq B\}
$$

for uma estrutura coarse sobre $X$. A partir disso, da observação 4.1.2 e da definição 4.1.1, torna-se simples demonstrar a proposição a seguir.

Proposição 4.1.8. Seja $X$ um conjunto não vazio. Nessas condições, uma coleção $\mathscr{B} \subseteq$ $\mathcal{P}(X \times X)$ será base de uma estrutura coarse sobre $X$ se, e somente se, possuir as seguintes propriedades:

a) Existe $B \in \mathscr{B}$ tal que $\Delta \subseteq B$;

b) Para quaisquer $B_{1}, B_{2} \in \mathscr{B}$, existe $E \in \mathscr{B}$ tal que $B_{1} \cup B_{2} \subseteq E$;

c) Para todo $B \in \mathscr{B}$, existe $E \in \mathscr{B}$ tal que $B^{-1} \subseteq E$; 
d) Para todo $B \in \mathscr{B}$, existe $E \in \mathscr{B}$ tal que $B \circ B \subseteq E$.

Antes de vermos alguns exemplos de estruturas coarse, façamos uma definição preliminar.

DeFINIÇÃo 4.1.9. Seja $(X, \tau)$ um espaço topológico. Dizemos que um subconjunto $E \subseteq X \times X$ é próprio em $(X, \tau)$ se $E[K]$ e $E^{-1}[K]$ forem relativamente compactos sempre que $K \subseteq X$ for relativamente compacto.

(Lembre-se de que $E[K]:=\{y \in X: \exists x \in K$ t.q. $(x, y) \in E\}$ e de que um conjunto $K \subseteq X$ é relativamente compacto em $(X, \tau)$ se, e somente se, $\bar{K}$ é compacto em $(X, \tau)$.)

Exemplos 4.1.10. Nos itens abaixo, consideremos um conjunto não vazio $X$.

a) A coleção $\mathcal{E}_{\max }:=\mathcal{P}(X \times X)$ é uma estrutura coarse maximal sobre $X$ (em relação à inclusão). Note que $\mathscr{B}_{\max }:=\{X \times X\}$ é uma base de $\mathcal{E}_{\max }$.

b) A coleção $\mathcal{E}_{\min }:=\mathcal{P}(\Delta(X))$ é uma estrutura coarse minimal sobre $X$ (também em relação à inclusão), e $\mathscr{B}_{\min }:=\{\Delta(X)\}$ é uma base de $\mathcal{E}_{\text {min }}$.

c) Sejam $Y \subseteq X$ um conjunto não vazio e $\mathcal{E}_{X}$ uma estrutura coarse sobre $X$. Nessas condições, é um exercício simples mostrar que

$$
\mathcal{E}_{Y}:=\left\{E \subseteq Y \times Y: E \in \mathcal{E}_{X}\right\}=\left\{E \cap(Y \times Y): E \in \mathcal{E}_{X}\right\}
$$

é uma estrutura coarse sobre $Y$. Dizemos que o par $\left(Y, \mathcal{E}_{Y}\right)$ é um subespaço coarse de $\left(X, \mathcal{E}_{X}\right)$. Note que, se $\mathscr{B}_{X}$ for uma base de $\mathcal{E}_{X}$, então $\mathscr{B}_{Y}=\left\{B \cap(Y \times Y): B \in \mathscr{B}_{X}\right\}$ será uma base de $\mathcal{E}_{Y}$.

d) A coleção $\mathcal{E}$ que consiste de todos os subconjuntos de $X \times X$ que possuem apenas um número finito de pontos fora da diagonal é uma estrutura coarse sobre $X$, a qual denominamos estrutura coarse discreta.

Demonstração. Observe que

$$
\mathcal{E}=\{E \subseteq X \times X: E \backslash \Delta \text { é finito }\}
$$

Vamos verificar que $\mathcal{E}$ satisfaz as condições de a) a e) da definição 4.1.1.

É claro que $\Delta \in \mathcal{E}$ (pois $\Delta \backslash \Delta=\emptyset$, que é finito). Por outro lado, se $E, F \in \mathcal{E}$, então $E \backslash \Delta$ e $F \backslash \Delta$ são finitos, do que segue que $(E \cup F) \backslash \Delta=(E \backslash \Delta) \cup(F \backslash \Delta)$ é finito e, portanto, $E \cup F \in \mathcal{E}$. Isso mostra que $\mathcal{E}$ satisfaz a) e b).

Para ver que $\mathcal{E}$ satisfaz c), basta notar que, para todo $E \subseteq X \times X, E^{-1} \backslash \Delta=(E \backslash \Delta)^{-1}$, a partir do que concluímos que $E \backslash \Delta$ será finito se, e somente se, $E^{-1} \backslash \Delta$ for finito. Disso segue que $E^{-1} \in \mathcal{E}$ para todo $E \in \mathcal{E}$. Além disso, é fácil ver que, se $F \subseteq E \subseteq X \times X$, então $F \backslash \Delta \subseteq E \backslash \Delta$. Como um subconjunto de um conjunto finito também é finito, disso resulta que todo subconjunto de um conjunto de $\mathcal{E}$ também pertence a $\mathcal{E}$.

Por fim, mostremos que $\mathcal{E}$ satisfaz d). Sejam $E, F \in \mathcal{E}$. Vamos mostrar que

$$
(E \circ F) \backslash \Delta \subseteq(E \backslash \Delta) \cup(F \backslash \Delta) \cup \pi_{1}(F \backslash \Delta) \times \pi_{2}(E \backslash \Delta),
$$

em que $\pi_{1}$ e $\pi_{2}$ denotam as projeções na primeira e na segunda coordenadas, respectivamente. Como $E \backslash \Delta$ e $F \backslash \Delta$ são finitos, disso resultará que $(E \circ F) \backslash \Delta$ é finito e, portanto, $E \circ F \in \mathcal{E}$. 
Seja $(x, y) \in(E \circ F) \backslash \Delta$ um ponto qualquer. Então $x \neq y$ e existe $z \in X$ tal que $(x, z) \in F$ e $(z, y) \in E$. Temos três possibilidades: ou $z=x$; ou $z=y$; ou $z \neq x$ e $z \neq y$.

Se $z=x$, então, como $(z, y) \in E$ e $x \neq y$, teremos que $(x, y) \in E \backslash \Delta$. Analogamente, $z=y$ implica $(x, y) \in F \backslash \Delta$. Por fim, se $z \neq x$ e $z \neq y$, teremos que $(x, z) \in F \backslash \Delta$ e $(z, y) \in E \backslash \Delta$, do que segue que $x \in \pi_{1}(F \backslash \Delta)$ e $y \in \pi_{2}(E \backslash \Delta)$, ou, de forma equivalente, $(x, y) \in \pi_{1}(F \backslash \Delta) \times \pi_{2}(E \backslash \Delta)$.

e) Dada uma topologia $\tau$ sobre $X$, dizemos que

$$
\mathcal{E}_{i}:=\{E \subseteq X \times X: E \text { é próprio em }(X, \tau)\} .
$$

é a estrutura coarse indiscreta associada a $(X, \tau)$. É imediato ver que, se $X$ for compacto, $\mathcal{E}_{i}=\mathcal{P}(X \times X)$.

Vamos provar, a seguir, que $\mathcal{E}_{i}$ é, de fato, uma estrutura coarse sobre $X$.

Demonstração. Primeiramente, note que, para todo $K \subseteq X, \Delta[K]=K=\Delta^{-1}[K]$ e, portanto, $\Delta$ é próprio em $(X, \tau)$.

Para mostrarmos que $\mathcal{E}_{i}$ satisfaz b) e d) da definição 4.1.1, consideremos subconjuntos $E, F \in$ $\mathcal{E}_{i}$ quaisquer. Seja $K \subseteq X$ um conjunto relativamente compacto. Como $E$ e $F$ são próprios, disso segue $\overline{E[K]}$ e $\overline{F[K]}$ são compactos e, portanto, $\overline{E[K]} \cup \overline{F[K]}$ é compacto. Note que

$$
\overline{(E \cup F)[K]}=\overline{E[K] \cup F[K]} \subseteq \overline{E[K]} \cup \overline{F[K]} .
$$

Como $\overline{(E \cup F)[K]}$ é um fechado contido em um compacto, concluímos que $\overline{(E \cup F)[K]}$ é compacto. Por outro lado, como $F[K]$ é relativamente compacto e $E$ é próprio, temos também que $\overline{(E \circ F)[K]}=\overline{E[F[K]]}$ é compacto.

Além disso, como $(E \cup F)^{-1}=E^{-1} \cup F^{-1}$ e $(E \circ F)^{-1}=F^{-1} \circ E^{-1}$, o mesmo raciocínio pode ser aplicado a $E^{-1}[K], F^{-1}[K]$ e $F^{-1}\left[E^{-1}[K]\right]$ para mostrar que $(E \cup F)^{-1}[K]$ e $(E \circ F)^{-1}[K]$ são relativamente compactos.

Por fim, como $K$ é um conjunto relativamente compacto qualquer, disso resulta que $E \cup F$ e $E \circ F$ são próprios em $(X, \tau)$.

A propriedade c) decorre trivialmente da definição 4.1 .9 e do fato de que $\left(E^{-1}\right)^{-1}=E$. Mostremos que $\mathcal{E}$ também satisfaz e).

Sejam $E \in \mathcal{E}_{i}, F$ um subconjunto de $E$ e $K \subseteq X$ um conjunto relativamente compacto. Como $\overline{E[K]}$ é compacto e

$$
F \subseteq E \Rightarrow F[K] \subseteq E[K] \Rightarrow \overline{F[K]} \subseteq \overline{E[K]}
$$

concluímos que $\overline{F[K]}$ é compacto (pois é um fechado contido em um compacto). Além disso, como $F \subseteq E$ se, e somente se, $F^{-1} \subseteq E^{-1}$, podemos aplicar o mesmo raciocício a $E^{-1}[K]$ e $F^{-1}[K]$ para concluírmos que $\overline{F^{-1}[K]}$ é compacto. Como o conjunto relativamente compacto $K$ é arbitrário, disso segue que $F$ é próprio em $(X, \tau)$.

Na seção 3.1, mostramos que uma pseudométrica $d$ sobre um conjunto $X$ induz uma uniformidade sobre $X$. A seguir, mostraremos que também podemos obter estruturas coarse sobre $X$ a partir de $d$. No próximo exemplo, apresentaremos uma das mais importantes, a qual será utilizada recorrentemente ao longo da dissertação. 
EXEMPLO 4.1.11 (ESTRUTURA COARSE LimitadA ASSOCIADA A UMA PSEUdométrica). Sejam $X$ um conjunto não vazio e $d$ uma pseudométrica sobre $X$. Nessas condições, a coleção

$$
\mathscr{B}_{d}:=\left\{E_{\alpha}^{d}: \alpha>0\right\}
$$

é base de uma estrutura coarse $\mathcal{E}_{d}$ sobre $X$, a qual denominamos estrutura coarse limitada associada à pseudométrica $d$.

Demonstração. Para concluírmos que $\mathscr{B}_{d}$ é base de uma estrutura coarse sobre $X$, basta verificarmos que $\mathscr{B}_{d}$ satisfaz as propriedades elencadas na proposição 4.1.8.

Segue diretamente do que foi feito no exemplo 3.1.12 que $\mathscr{B}_{d}$ satisfaz a), c) e d), bastando substituir $\varepsilon$ por $2 \alpha$ na verificação da propriedade d). E, sendo assim, basta mostrarmos que $\mathscr{B}_{d}$ satisfaz b). Para isso, tomemos $\alpha_{1}, \alpha_{2}>0$ de modo arbitrário, e definamos $\alpha:=\max \left\{\alpha_{1}, \alpha_{2}\right\}$. Observe que, para todos $x, y \in X$,

$$
(x, y) \in E_{\alpha_{1}}^{d} \cup E_{\alpha_{2}}^{d} \Leftrightarrow d(x, y)<\alpha_{1} \text { ou } d(x, y)<\alpha_{2} \Leftrightarrow d(x, y)<\alpha \Leftrightarrow(x, y) \in E_{\alpha}^{d} .
$$

Assim sendo, $E_{\alpha_{1}}^{d} \cup E_{\alpha_{2}}^{d}=E_{\alpha}^{d}$. E, como $\alpha_{1}, \alpha_{2}>0$ são arbitrários, disso concluímos que $B_{1} \cup B_{2} \in \mathscr{B}_{d}$ quaisquer que sejam $B_{1}, B_{2} \in \mathscr{B}_{d}$.

NotAÇÃo: A estrutura coarse limitada associada a uma pseudométrica $d$ sobre um conjunto $X$ será denotada por $\mathcal{E}_{d}$.

OBSERVAÇÃo 4.1.12. Dado um espaço pseudométrico $(X, d)$, não é difícil mostrar que

$$
\mathcal{E}_{d}=\{E \subseteq X \times X:\{d(x, y):(x, y) \in E\} \text { é limitado }\} .
$$

(Observe que, em particular, isso justifica o uso do termo "limitada" para designar essa estrutura coarse.)

As figuras 4.1 e 4.2 ilustram conjuntos controlados genéricos da estrutura coarse limitada associada à métrica usual de $\mathbb{R}$.

ObSERVAÇÃo 4.1.13. Dados um espaço pseudométrico $(X, d)$ e um subconjunto não vazio $A \subseteq$ $X$, é imediato ver que $\left(\mathcal{E}_{d}\right)_{A}=\mathcal{E}_{\left.d\right|_{A \times A}}$. Isso é análogo ao que ocorre com a uniformidade induzida pela restrição de uma pseudométrica a um subconjunto não vazio (veja a observação 3.1.13), e segue diretamente do exemplo 4.1.10-c) e do fato de que, para cada $\alpha>0$,

$$
E_{\alpha}^{d} \cap(A \times A)=E_{\alpha}^{\left.d\right|_{A \times A}} .
$$

OBSERVAÇÃo 4.1.14. Assim como no caso das estruturas uniformes, é fácil ver que duas pseudométricas sobre um mesmo conjunto $X$ podem induzir a mesma estrutura coarse sobre $X$, e bastam alguns segundos de reflexão para concluírmos que o exemplo dado na observação 3.1.15 também funciona neste caso.

A proposição seguinte é uma adapação da proposição 3.1.16 para o caso das estruturas coarse, e estabelece uma condição suficiente para que duas pseudométricas sobre um mesmo conjunto $X$ induzam a mesma estrutura coarse limitada sobre $X$.

Proposição 4.1.15. Sejam $d_{1}$ e $d_{2}$ duas pseudométricas sobre um mesmo conjunto $X$, e suponhamos que existam funções $\psi_{1}:\left[0,+\infty\left[\longrightarrow\left[0,+\infty\left[\right.\right.\right.\right.$ e $\psi_{2}:[0,+\infty[\longrightarrow[0,+\infty[$, com $\psi_{1}(0)=\psi_{2}(0)=0$, e tais que, para todo $(x, y) \in X \times X, \psi_{1}\left(d_{1}(x, y)\right) \leq d_{2}(x, y) e$ $\psi_{2}\left(d_{2}(x, y)\right) \leq d_{1}(x, y)$. Suponhamos ainda que, para cada $i \in\{1,2\}$ e cada $M>0$, exista 


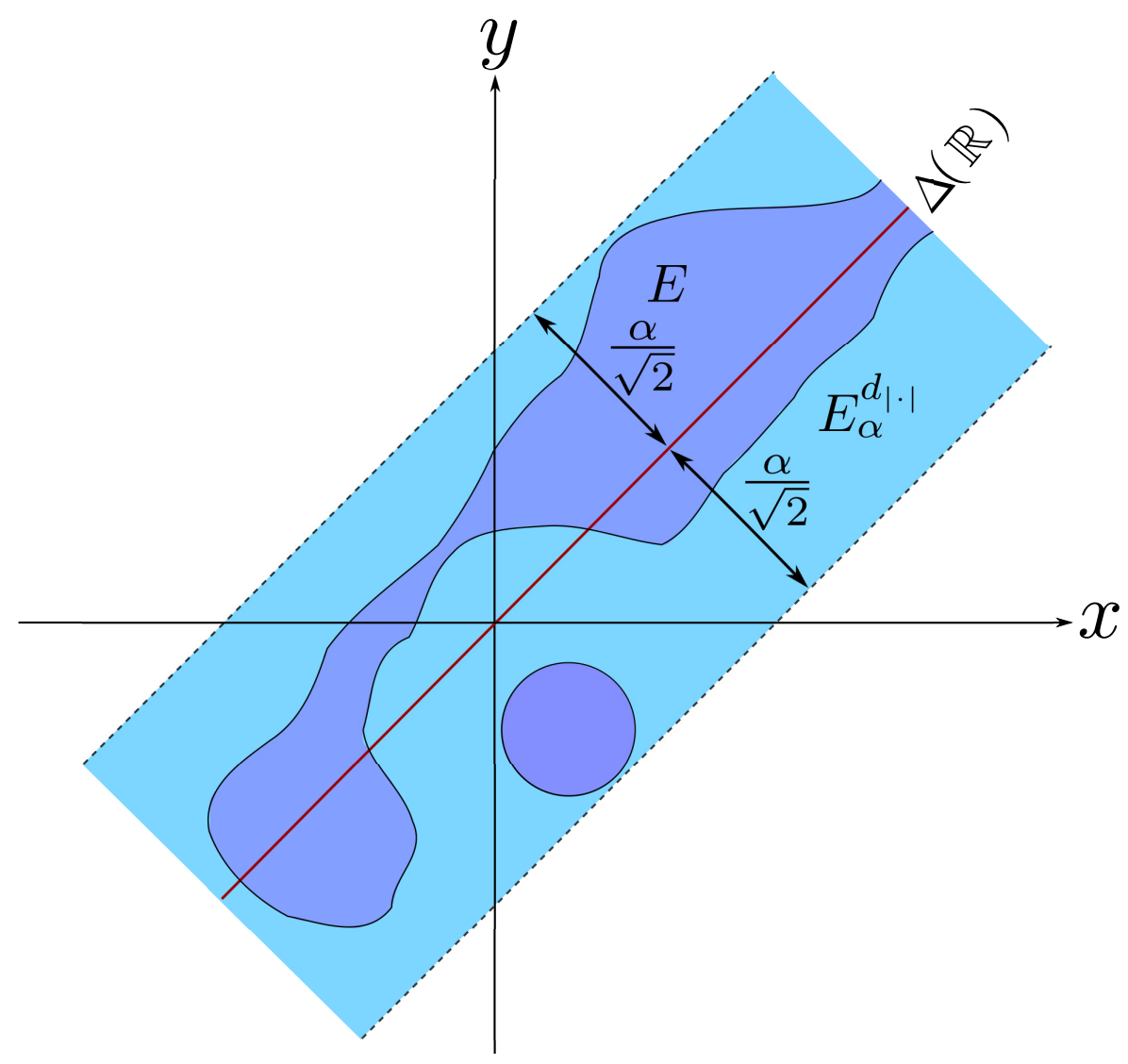

Figura 4.1: Conjunto controlado da estrutura coarse usual sobre $\mathbb{R}$.

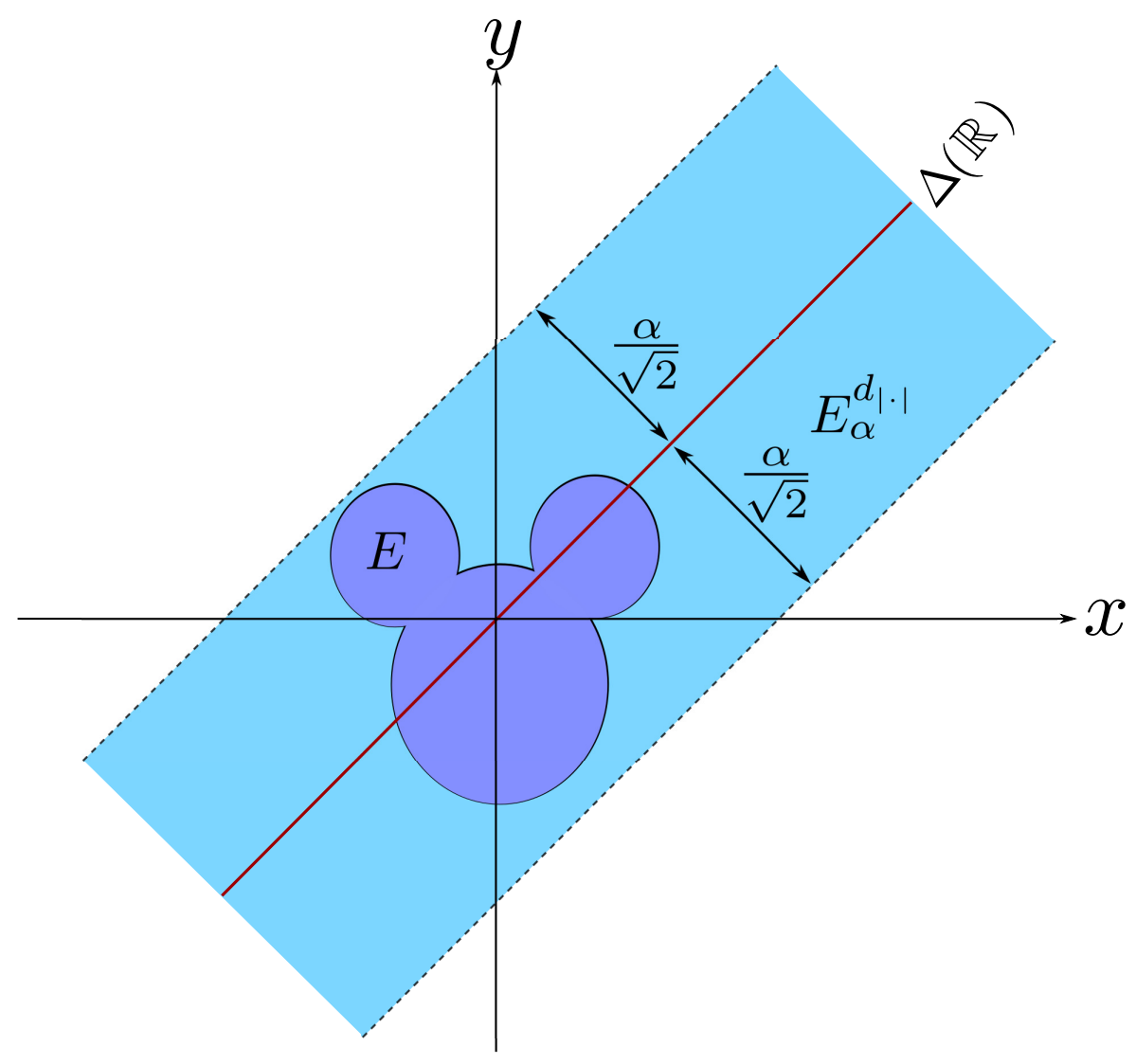

Figura 4.2: Um outro exemplo de conjunto controlado. 
$\Delta_{i}>0$ tal que $\psi_{i}\left(d_{i}(x, y)\right) \geq M$ para todos $x, y \in X$ tais que $d_{i}(x, y) \geq \Delta_{i}$. Nessas condições, as estruturas coarse limitadas associadas a $d_{1}$ e a $d_{2}$ coincidem.

Demonstração. Essa demonstração é, essencialmente, análoga à da proposição 3.1.16, com as devidas adaptações. A ideia é mostrar que, para cada $M>0$, existe $\Delta>0$ tal que $E_{M}^{d_{1}} \subseteq E_{\Delta}^{d_{2}}$. Como $\mathscr{B}_{1}:=\left\{E_{M}^{d_{1}}: M>0\right\}$ e $\mathscr{B}_{2}:=\left\{E_{\Delta}^{d_{2}}: \Delta>0\right\}$ são bases de $\mathcal{E}_{d_{1}}$ e de $\mathcal{E}_{d_{2}}$, respectivamente, disso seguirá que $\mathcal{E}_{d_{1}} \subseteq \mathcal{E}_{d_{2}}$ (pela proposição 4.1.7). Feito isso, bastará invertermos os papéis de 1 e de 2 na demonstração e repetirmos o argumento para obtermos a inclusão oposta.

Seja $M>0$ dado. Por hipótese, podemos fixar $\Delta_{2}>0$ tal que $\psi_{2}\left(d_{2}(x, y)\right) \geq M$ para todos $x, y \in X$ tais que $d_{2}(x, y) \geq \Delta_{2}$. Dessa forma, se $x, y \in X$ forem tais que $\psi_{2}\left(d_{2}(x, y)\right)<M$, teremos, necessariamente, $d_{2}(x, y)<\Delta_{2}$. Como $\psi_{2}\left(d_{2}(x, y)\right) \leq d_{1}(x, y)$, disso resulta que, para todo $(x, y) \in X \times X$,

$$
(x, y) \in E_{M}^{d_{1}} \Rightarrow d_{1}(x, y)<M \Rightarrow \psi_{2}\left(d_{2}(x, y)\right)<M \Rightarrow d_{2}(x, y)<\Delta_{2} \Rightarrow(x, y) \in E_{\Delta_{2}}^{d_{2}} .
$$

E, por sua vez, isso mostra que $E_{M}^{d_{1}} \subseteq E_{\Delta_{2}}^{d_{2}}$.

No corolário 3.1.17, mostramos que, se $d$ é uma pseudométrica sobre um conjunto $X$, então $\sqrt{d}$ também é uma pseudométrica sobre $X$ e ambas induzem sobre $X$ a mesma estrutura uniforme. Vejamos, agora, o que a proposição 4.1.15 nos permite deduzir a respeito das estruturas coarse limitadas associadas a $d$ e a $\sqrt{d}$.

Corolário 4.1.16. Dada uma pseudométrica $d_{1}$ sobre um conjunto $X$, consideremos a pseudométrica $d_{2}: X \times X \longrightarrow\left[0,+\infty\left[\right.\right.$ tal que $d_{2}(x, y)=\sqrt{d_{1}(x, y)}$. Nessas condições, $d_{1}$ e $d_{2}$ induzem sobre $X$ a mesma estrutura coarse limitada.

Demonstração. Sejam $\psi_{1}, \psi_{2}:\left[0,+\infty\left[\longrightarrow\left[0,+\infty\left[\right.\right.\right.\right.$ tais que $\psi_{1}(u)=\sqrt{u}$ e $\psi_{2}(u)=u^{2}$. Na demonstração do corolário 3.1.17, já verificamos que $\psi_{1}(0)=\psi_{2}(0)=0$ e que, para todo $(x, y) \in X \times X$

$$
\psi_{1}\left(d_{1}(x, y)\right)=d_{2}(x, y) \text { e } \psi_{2}\left(d_{2}(x, y)\right)=d_{1}(x, y) .
$$

Note ainda que, se $M>0$ é arbitrário, então, tomando $\Delta_{1}=M^{2}, \Delta_{2}=\sqrt{M}$ e $u \in[0,+\infty[$, teremos que

$$
u \geq \Delta_{1} \Rightarrow u \geq M^{2} \Rightarrow \sqrt{u} \geq M \Rightarrow \psi_{1}(u) \geq M
$$

$\mathrm{e}$

$$
u \geq \Delta_{2} \Rightarrow u \geq \sqrt{M} \Rightarrow u^{2} \geq M \Rightarrow \psi_{2}(u) \geq M .
$$

Logo, segue da proposição 4.1 .15 que $\mathcal{E}_{d_{1}}=\mathcal{E}_{d_{2}}$.

Para finalizar, vamos citar um segundo exemplo de estrutura coarse a qual pode ser obtida a partir de uma pseudométrica.

EXemplo 4.1.17 (Estrutura coarse $\mathcal{C}_{0}$ ). Sejam $(X, \tau)$ um espaço topológico e $d$ uma pseudométrica sobre $X$. A estrutura coarse $\mathcal{C}_{0}$ associada a $d$ é a coleção $\mathcal{E}$ dos subconjuntos $E \subseteq X \times X$ tais que, para todo $\varepsilon>0$, existe $K \subseteq X$ compacto em $(X, \tau)$ tal que $d(x, y)<\varepsilon$ para todo $(x, y) \in E \backslash(K \times K)$. Mais detalhes a respeito dessa estrutura coarse podem ser encontrados nas referências [Wri02] e [Wri03]. 


\subsection{A Estrutura COARSE GERAdA POR UMA COLEÇÃo}

A seguir, vamos introduzir o importante conceito de estrutura coarse gerada por uma coleção de subconjuntos. Mais especificamente, vamos mostrar como obter uma estrutura coarse sobre um conjunto não vazio $X$ a partir de uma coleção de subconjuntos de $X \times X$. Para isso, começaremos mostrando três resultados simples, mas importantes, os quais decorrem diretamente das definições de estrutura coarse e de base. Ao longo desta seção, estaremos sempre trabalhando com um conjunto não vazio $X$.

Proposição 4.2.1. A intersecção de estruturas coarse sobre X é uma estrutura coarse sobre $X$.

Demonstração. Sejam $I$ um conjunto não vazio e $\left(\mathcal{E}_{i}\right)_{i \in I}$ uma família de estruturas coarse sobre $X$. Vamos mostrar que $\mathcal{E}=\bigcap_{i \in I} \mathcal{E}_{i}$ é, também, uma estrutura coarse sobre $X$.

Primeiramente, observe que $\Delta \in \mathcal{E}$, pois $\Delta \in \mathcal{E}_{i}$ para todo $i \in I$. Além disso, dados quaisquer $E, F \subseteq X \times X$, é imediato ver que

$$
E, F \in \mathcal{E} \Rightarrow E, F \in \mathcal{E}_{i} \quad \forall i \in I \Rightarrow E^{-1}, E \cup F, E \circ F \in \mathcal{E}_{i} \quad \forall i \in I \Rightarrow E^{-1}, E \cup F, E \circ F \in \mathcal{E} .
$$

Analogamente, se $F \subseteq E \subseteq X \times X$, então

$$
E \in \mathcal{E} \Rightarrow E \in \mathcal{E}_{i} \quad \forall i \in I \Rightarrow F \in \mathcal{E}_{i} \quad \forall i \in I \Rightarrow F \in \mathcal{E}
$$

o que conclui a prova de que $\mathcal{E}$ é uma estrutura coarse sobre $X$.

OBSERVAÇÃo 4.2.2. Ao contrário do que ocorre com a intersecção de estruturas coarse, a união de estruturas uniformes não é, em geral, uma estrutura uniforme, uma vez que, dada uma família não vazia $\left(\mathscr{D}_{i}\right)_{i \in I}$ de uniformidades sobre $X$, não é sempre verdade que $E \cap D \in \bigcup_{i \in I} D_{i}$ para todos $E, D \in \bigcup_{i \in I} D_{i}$.

Tendo em vista a proposição 4.2.1, é natural conjecturar que podemos construir uma base para a intersecção de uma família não vazia $\left(\mathcal{E}_{i}\right)_{i \in I}$ de estruturas coarse sobre $X$ a partir de uma família de bases $\left(\mathscr{B}_{i}\right)_{i \in I}$, em que, para cada $i \in I, \mathscr{B}_{i}$ é uma base de $\mathcal{E}_{i}$. Se o leitor acredita que isso é possível, ele está de parabéns! Sua intuição está correta. E, nas próximas linhas, mostraremos como fazer isso.

Proposição 4.2.3. Seja $\left(\mathcal{E}_{i}\right)_{i \in I}$ uma família não vazia de estruturas coarse sobre $X$ e, para cada $i \in I$, seja $\mathscr{B}_{i}$ uma base de $\mathcal{E}_{i}$. Nessas condições, a coleção

$$
\mathscr{B}:=\left\{B \subseteq X \times X: \exists\left(B_{i}\right)_{i \in I} \text { t.q. } B_{i} \in \mathscr{B}_{i} \text { para todo } i \in I \text { e } B=\bigcap_{i \in I} B_{i}\right\}
$$

é uma base de $\mathcal{E}$, em que $\mathcal{E}=\bigcap_{i \in I} \mathcal{E}_{i}$.

Demonstração. Para provarmos que $\mathscr{B}$ é uma base de $\mathcal{E}$, devemos mostrar que $\mathscr{B} \subseteq \mathcal{E}$ e que cada elemento de $\mathcal{E}$ está contido em algum elemento de $\mathscr{B}$. E é o que faremos a seguir.

Seja $B \in \mathscr{B}$ dado. Por definição, disso segue que existe $\left(B_{i}\right)_{i \in I}$ tal que $B_{i} \in \mathcal{E}_{i}$ para todo $i \in I$ e $B=\bigcap_{i \in I} B_{i}$. Observe que

$$
\left(B_{i} \in \mathcal{E}_{i} \text { e } B \subseteq B_{i}\right) \quad \forall i \in I \Rightarrow B \in \mathcal{E}_{i} \quad \forall i \in I \Rightarrow B \in \bigcap_{i \in I} \mathcal{E}_{i} \Rightarrow B \in \mathcal{E}
$$


E, como $B \in \mathscr{B}$ é arbitrário, disso resulta que $\mathscr{B} \subseteq \mathcal{E}$.

Tomemos, agora, um $E \in \mathcal{E}$ qualquer. Como $\mathcal{E}=\bigcap_{i \in I} \mathcal{E}_{i}$, temos que $E \in \mathcal{E}_{i}$ para todo $i \in I$. E como, para cada $i \in I, \mathscr{B}_{i}$ é uma base de $\mathcal{E}_{i}$, disso segue que, para cada $i \in I$, existe $B_{i} \in \mathscr{B}_{i}$ tal que $E \subseteq B_{i}$. Observe que

$$
E \subseteq B_{i} \quad \forall i \in I \Rightarrow E \subseteq \bigcap_{i \in I} B_{i}
$$

Como $\bigcap_{i \in I} B_{i} \in \mathscr{B}$, disso concluímos que $E$ está contido em um elemento de $\mathscr{B}$.

Vejamos, agora, uma consequência simples da proposição 4.2.1.

Proposição 4.2.4. Dada uma coleção arbitrária $\mathcal{S}$ de subconjuntos de $X \times X$, existe uma única estrutura coarse sobre $X$ que contém $\mathcal{S}$ e é mais fina do que qualquer outra estrutura coarse sobre $X$ contendo $\mathcal{S}$.

Demonstração. Seja $\mathscr{E}$ o conjunto de todas as estruturas coarse sobre $X$ que contêm $\mathcal{S}$. Note que $\mathscr{E}$ é não vazio, pois $\mathcal{P}(X \times X) \in \mathscr{E}$. Logo, segue da proposição 4.2 .1 que $\mathcal{E}=\bigcap_{\mathcal{E}^{\prime} \in \mathscr{E}} \mathcal{E}^{\prime}$ é uma estrutura coarse sobre $X$. Note ainda que $\mathcal{S} \subseteq \mathcal{E}$ (pois $\mathcal{S} \subseteq \mathcal{E}^{\prime}$ para todo $\mathcal{E}^{\prime} \in \mathscr{E}$ ) e que $\mathcal{E}$ está contido em toda estrutura coarse sobre $X$ que contém $\mathcal{S}$ (por definição). A unicidade de $\mathcal{E}$ decorre imediatamente da observação de que, se $\mathcal{F}$ é outra estrutura coarse sobre $X$ com as propriedades do enunciado, então $\mathcal{F} \subseteq \mathcal{E}$ (pois $\mathcal{S} \subseteq \mathcal{E}$ ) e $\mathcal{E} \subseteq \mathcal{F}$ (pois $\mathcal{S} \subseteq \mathcal{F}$ ), e, portanto, $\mathcal{F}=\mathcal{E}$.

A proposição 4.2.4 e as ideias envolvidas em sua demonstração motivam a seguinte definição:

Definição 4.2.5. Seja $\mathcal{S}$ uma coleção arbitrária de subconjuntos de $X \times X$. Chamamos de estrutura coarse gerada por $\mathcal{S}$ à intersecção de todas as estruturas coarse sobre $X$ que contêm $\mathcal{S}$.

(Note que, pela proposição 4.2.4, a estrutura coarse gerada por $\mathcal{S}$ é mais fina do que qualquer outra estrutura coarse sobre $X$ contendo $\mathcal{S}$.)

NotAÇÃO: Denotaremos por $\mathcal{E}_{\mathcal{S}}$ a estrutura coarse gerada por uma coleção $\mathcal{S}$ de subconjuntos de $X \times X$.

OBSERVAÇÃo 4.2.6. É claro que uma coleção $\mathcal{S} \subseteq \mathcal{P}(X \times X)$ não precisa ser, necessariamente, uma base de $\mathcal{E}_{\mathcal{S}}$. Para ilustrar isso, consideremos um conjunto $X$ não vazio, e tomemos $\mathcal{S}:=$ $\{\{(x, x)\}\}$, em que $x \in X$. Nesse caso, $\mathcal{E}_{\mathcal{S}}=\mathcal{P}(\Delta)$ (pois $\mathcal{E}_{\mathcal{S}} \subseteq \mathcal{P}(\Delta)$ e $\mathcal{P}(\Delta)$ é uma estrutura coarse minimal sobre $X$ ), e $\mathcal{S}$ não é, obviamente, uma base de $\mathcal{E}_{\mathcal{S}}$. No entanto, é fácil ver que, se $\mathscr{B}$ for uma base de uma estrutura coarse $\mathcal{E}$ sobre $X$, então $\mathcal{E}_{\mathscr{B}}=\mathcal{E}$.

Note ainda que, se $\mathcal{S}_{1}$ e $\mathcal{S}_{2}$ forem coleções de subconjuntos de $X \times X$ tais que $\mathcal{S}_{1} \subseteq \mathcal{S}_{2}$, então $\mathcal{E}_{\mathcal{S}_{1}} \subseteq \mathcal{E}_{\mathcal{S}_{2}}$.

O próximo resultado, com o qual encerramos a seção, nos permite descrever os elementos da estrutura coarse gerada por uma coleção $\mathcal{S} \subseteq \mathcal{P}(X \times X)$ em função dos elementos de $\mathcal{S}$.

Proposição 4.2.7. Para cada $\mathcal{S} \subseteq \mathcal{P}(X \times X)$, a estrutura coarse gerada por $\mathcal{S}$ tem como base a coleção

$$
\begin{aligned}
\mathscr{B}_{\mathcal{S}}:=\{ & F_{1} \circ \ldots \circ F_{n} \subseteq X \times X: n \in \mathbb{N} \text { e, para cada } i \in\{1, \ldots, n\}, \\
& \left.F_{i} \text { é uma reunião finita de elementos de }\{\Delta\} \cup \mathcal{S} \cup \mathcal{S}^{-1}\right\},
\end{aligned}
$$

em que $\mathcal{S}^{-1}:=\left\{F^{-1}: F \in \mathcal{S}\right\}$ 
Demonstração. Seja $\mathcal{S} \subseteq \mathcal{P}(X \times X)$ dada. Vamos começar mostrando que $\mathscr{B}_{\mathcal{S}}$ é base de uma estrutura coarse sobre $X$. Para isso, basta verificarmos que $\mathscr{B}_{\mathcal{S}}$ satisfaz as condições da proposição 4.1.8.

Inicialmente, note que $\Delta \in \mathscr{B}_{\mathcal{S}}$ e que $B \circ B$ pertence a $\mathscr{B}_{\mathcal{S}}$ para todo $B \in \mathscr{B}_{\mathcal{S}}$ (pela associatividade de $\circ$ ).

Observe ainda que, se $F$ e $G$ são reuniões finitas de elementos de $\{\Delta\} \cup \mathcal{S} \cup \mathcal{S}^{-1}$, então $F^{-1}$ e $F \cup G$ também o são. Isso será útil para provarmos que $\mathscr{B}_{\mathcal{S}}$ satisfaz as duas condições restantes.

Com efeito, segue da observação acima que, se $B=F_{1} \circ \ldots \circ F_{n}$ pertence a $\mathscr{B}_{\mathcal{S}}$, então $B^{-1}=\left(F_{1} \circ \ldots \circ F_{n}\right)^{-1}=F_{n}^{-1} \circ \ldots \circ F_{1}^{-1}$ também está em $\mathscr{B}_{\mathcal{S}}$.

Por fim, mostremos que, se $B=F_{1} \circ \ldots \circ F_{n}$ e $B^{\prime}=G_{1} \circ \ldots \circ G_{m}$ estão em $\mathscr{B}_{\mathcal{S}}$, então existe $E \in \mathscr{B}_{\mathcal{S}}$ tal que $B \cup B^{\prime} \subseteq E$. Suponhamos, sem perda de generalidade, que $n \geq m$. Se $n=m$, segue diretamente da proposição 2.2.4 que

$$
B \cup B^{\prime}=\left(F_{1} \circ \ldots \circ F_{n}\right) \cup\left(G_{1} \circ \ldots \circ G_{n}\right) \subseteq\left(F_{1} \cup G_{1}\right) \circ \ldots \circ\left(F_{n} \cup G_{n}\right) \in \mathscr{B}_{\mathcal{S}} .
$$

Já no caso em que $n>m$, temos que

$$
\begin{aligned}
B \cup B^{\prime} & =\left(F_{1} \circ \ldots \circ F_{n}\right) \cup\left(G_{1} \circ \ldots \circ G_{m}\right)=\left(F_{1} \circ \ldots \circ F_{n}\right) \cup(G_{1} \circ \ldots \circ G_{m} \circ \underbrace{\Delta \circ \ldots \circ \Delta}_{n-m \text { vezes }}) \\
& \subseteq\left(F_{1} \cup G_{1}\right) \circ \ldots \circ\left(F_{m} \cup G_{m}\right) \circ\left(F_{m+1} \cup \Delta\right) \circ \ldots \circ\left(F_{n} \cup \Delta\right) \in \mathscr{B}_{\mathcal{S}} .
\end{aligned}
$$

Isso completa a demonstração. Seja $\mathcal{E}$ a estrutura coarse gerada por $\mathscr{B}_{\mathcal{S}}$. Como $\mathcal{S} \subseteq \mathscr{B}_{\mathcal{S}} \subseteq \mathcal{E}$, segue da definição de $\mathcal{E}_{\mathcal{S}}$ que $\mathcal{E}_{\mathcal{S}} \subseteq \mathcal{E}$. Por outro lado, como $\mathcal{S} \subseteq \mathcal{E}_{\mathcal{S}}$, decorre das propriedades das estruturas coarse que $\mathscr{B}_{\mathcal{S}} \subseteq \mathcal{E}_{\mathcal{S}}$ e, portanto, $\mathcal{E} \subseteq \mathcal{E}_{\mathcal{S}}$, a partir do que concluímos que $\mathcal{E}_{\mathcal{S}}=\mathcal{E}$.

\subsection{A estrutura COARse À ESquerda DE UM GRUPo TO- POLÓGICO}

Nesta seção, vamos apresentar a chamada estrutura coarse à esquerda ${ }^{3}$ de um grupo topológico, introduzida por Christian Rosendal em um manuscrito de 2017 [Ros17a] e discutida pouco depois em um artigo do mesmo ano [Ros17b]. Para isso, precisaremos, antes, fazer uma definição preliminar.

Definição 4.3.1. Dado um grupo topológico $(G, \cdot, \tau)$, dizemos que uma pseudométrica $d$ sobre $G$ é contínua em $(G, \cdot, \tau)$ se for contínua em relação a $\tau_{\text {prod }}$, em que $\tau_{\text {prod }}$ é a topologia produto induzida por $\tau$ sobre $G \times G$. Quando a topologia $\tau$ estiver clara pelo contexto, diremos apenas que $d$ é contínua.

Sejam $(G, \cdot, \tau)$ um grupo topológico, $\mathcal{U}_{L}$ a estrutura uniforme à esquerda associada a $(G, \cdot, \tau)$ e $\mathcal{D}$ a coleção de todas as pseudométricas sobre $G$ que são contínuas em $(G, \cdot, \tau)$ e invariantes à esquerda em $(G, \cdot)$. Em seu livro Sur les Espaces à Structure Uniforme et sur la Topologie Générale [Wei37], de 1937, André Weil provou que $\mathcal{U}_{L}=\bigcup_{d \in \mathcal{D}} \mathscr{D}_{d}$, em que, para cada $d \in \mathcal{D}$, $\mathscr{D}_{d}$ é a estrutura uniforme induzida por $d$ sobre $G .{ }^{4}$ Dessa forma, um subconjunto $D \subseteq G \times G$ pertence a $\mathcal{U}_{L}$ se, e somente, se, existem $d \in \mathcal{D}$ e $\varepsilon>0$ tais que $D_{\varepsilon}^{d} \subseteq D$.

\footnotetext{
${ }^{3}$ Do inglês left-coarse structure.

${ }^{4}$ Observe que, em particular, disso segue que $\mathcal{D} \neq \emptyset$.
} 
O resultado de Weil nos mostra que $\mathcal{U}_{L}$ é a estrutura uniforme mais grosseira (isto é, menos fina) que refina todas as $\mathscr{D}_{d}$, em que $d \in \mathcal{D}$. Ou, em outras palavras, $\mathcal{U}_{L}$ é o refinamento comum mais grosseiro para a coleção $\left\{\mathscr{D}_{d}: d \in \mathcal{D}\right\}$. Para estabelecer uma conexão entre esse resultado e o que sabemos sobre estruturas coarse, poderíamos, agora, nos perguntar qual é a estrutura coarse mais grosseira que refina todas as estruturas coarse limitadas induzidas por pseudométricas em $\mathcal{D}$. Tendo em vista a definição 4.1 .3 e a proposição 4.2.1, fica claro que, nesse caso, $\mathcal{E}:=\bigcap_{d \in \mathcal{D}} \mathcal{E}_{d}$ é o refinamento que procuramos. Dessa forma, $\mathcal{E}$ desempenha um papel análogo, no contexto das estruturas coarse, ao desempenhado pela estrutura uniforme à esquerda no contexto das estruturas uniformes. Isso motiva a seguinte definição:

DefiniçÃo 4.3.2 (Estrutura COARSE À ESQUERDA). A estrutura coarse à esquerda de um grupo topológico $(G, \cdot, \tau)$ é a coleção

$$
\mathcal{E}_{L}:=\bigcap_{d \in \mathcal{D}} \mathcal{E}_{d}
$$

em que $\mathcal{D}$ é a coleção das pseudométricas sobre $G$ que são contínuas em $(G, \cdot, \tau)$ e invariantes à esquerda em $(G, \cdot)$ e, para cada $d \in \mathcal{D}, \mathcal{E}_{d}$ é a estrutura coarse limitada associada a $d$.

Observe que um subconjunto $E \subseteq G \times G$ pertence a $\mathcal{E}_{L}$ se, e somente se, para todo $d \in \mathcal{D}$, existe $\varepsilon>0$ tal que $d(x, y)<\varepsilon$ para todo $(x, y) \in E$.

A definição da estrutura coarse à esquerda traduz a ideia de que, embora $(G, \cdot \tau)$ possa ter, em $\mathcal{D}$, várias pseudométricas compatíveis (isto é, várias pseudométricas que induzem a topologia do grupo), sua geometria de larga escala, em geral, não depende da escolha particular de uma ou de outra dessas pseudométricas, podendo ser descrita apenas em termos de propriedades comuns a todas elas.

OBSERVAÇÃo 4.3.3. Pode-se mostrar que, assim como acontece com a uniformidade à esquerda, a estrutura coarse à esquerda associada ao grupo aditivo de um espaço vetorial normado coincide com a estrutura coarse limitada induzida pela norma. No capítulo 6, discutiremos, em detalhes, esse resultado, cuja demonstração está longe de ser óbvia.

\subsection{FunÇÕES ENTRE ESPAÇOS COARSE}

A seguir, estudaremos algumas classes especiais de funções entre espaços coarse e estabeleceremos relações entre elas. Ao longo desta seção, salvo menção em contrário, $(X, \mathcal{E})$ e $(Y, \mathcal{F})$ denotarão espaços coarse quaisquer.

Os primeiros conceitos que vamos estudar são os de bornologia e de função bornológica. Ao longo do texto, veremos que, do mesmo modo como as funções uniformente contínuas são os morfismos entre espaços uniformes, as funções bornológicas são os morfismos entre espaços coarse.

Definição 4.4.1 (Bornologia). Dizemos que uma função $f: X \longrightarrow Y$ é bornológica em relação a $\mathcal{E}$ e $\mathcal{F}$ se, para cada $E \in \mathcal{E}$, existir $F \in \mathcal{F}$ tal que $(f(x), f(y)) \in F$ para todo $(x, y) \in E$. Quando as estruturas coarse estiverem claras pelo contexto, diremos apenas que $f$ é bornológica. 
OBSERVAÇÃo 4.4.2. Sejam $\mathscr{B}$ e $\mathscr{C}$ bases de $\mathcal{E}$ e de $\mathcal{F}$, respectivamente, e $f: X \longrightarrow Y$ uma função. Note que, para mostrar que $f$ é bornológica, basta mostrar que, para todo $B \in \mathscr{B}$, existe $F \in \mathcal{F}$ tal que $(f(x), f(y)) \in F$ para todo $(x, y) \in B$. Isso segue do fato de que todo $E \in \mathcal{E}$ está contido em algum elemento de $\mathscr{B}$. Além disso, como cada $F \in \mathcal{F}$ está contido em um elemento de $\mathscr{C}$, é fácil constatar que, se $f$ for bornológica, então, para cada $E \in \mathcal{E}$, existe $C \in \mathscr{C}$ tal que $(f(x), f(y)) \in C$ para todo $(x, y) \in E$.

Vejamos, abaixo, alguns exemplos de funções bornológicas, assim como uma caracterização das funções bornológicas entre espaços pseudométricos.

EXEMPLOS 4.4.3.

a) Se $\mathcal{F}=\mathcal{P}(Y \times Y)$, toda função $f: X \longrightarrow Y$ é bornológica.

b) Se $\mathcal{E}=\mathcal{P}(\Delta(X))$, toda função $f: X \longrightarrow Y$ é bornológica. Para constatar isso, basta notar que $\Delta(Y) \in \mathcal{F}$ e que, para cada $E \in \mathcal{E}$ e cada $(x, y) \in X \times X$

$$
(x, y) \in E \Rightarrow(x, y) \in \Delta(X) \Rightarrow x=y \Rightarrow f(x)=f(y) \Rightarrow(f(x), f(y)) \in \Delta(Y) .
$$

c) Se $Z \subseteq X$ é um conjunto não vazio e $f: X \longrightarrow Y$ é bornológica em relação a $\mathcal{E}$ e $\mathcal{F}$, então a restrição de $f$ a $Z$ é bornológica em relação a $\mathcal{E}_{Z}$ e $\mathcal{F}$.

(Isso decorre imediatamente do fato de que $\mathcal{E}_{Z}:=\{E \subseteq Z \times Z: E \in \mathcal{E}\} \subseteq \mathcal{E}$.)

d) Suponhamos que $\mathcal{E}$ e $\mathcal{F}$ sejam induzidas por pseudométricas $d$ e $\partial$, respectivamente. Vamos mostrar que, nesse caso, uma função $f: X \longrightarrow Y$ é bornológica se, e somente se, para todo $\Delta>0$, existe $M>0$ tal que $\partial(f(x), f(y))<M$ para todos $x, y \in X$ tais que $d(x, y)<\Delta$. Para isso, consideremos as bases $\mathscr{B}:=\left\{E_{\Delta}^{d}: \Delta>0\right\}$ e $\mathscr{C}:=\left\{F_{M}^{\partial}: M>0\right\}$ de $\mathcal{E}$ e de $\mathcal{F}$, respectivamente.

$(\Rightarrow)$ Seja $\Delta>0$ dado. Como $f$ é bornológica e $\mathscr{C}$ é base de $\mathcal{F}$, segue da observação 4.4 .2 que podemos fixar $M>0$ de modo que $(f(x), f(y)) \in F_{M}^{\partial}$ para todo $(x, y) \in E_{\Delta}^{d}$. Dessa forma, para todos $x, y \in X$,

$$
d(x, y)<\Delta \Rightarrow(x, y) \in E_{\Delta}^{d} \Rightarrow(f(x), f(y)) \in F_{M}^{\partial} \Rightarrow \partial(f(x), f(y))<M .
$$

$(\Leftarrow)$ Seja $E \in \mathcal{E}$ dado. Como $\mathscr{B}$ é base de $\mathcal{E}$, podemos tomar $\Delta>0$ de modo que $E \subseteq E_{\Delta}^{d}$. Por hipótese, existe $M>0$ tal que $\partial(f(x), f(y))<M$ para todos $x, y \in X$ tais que $d(x, y)<\Delta$. Disso segue, em particular, que, para todos $x, y \in X$,

$$
(x, y) \in E \Rightarrow(x, y) \in E_{\Delta}^{d} \Rightarrow d(x, y)<\Delta \Rightarrow \partial(f(x), f(y))<M \Rightarrow(f(x), f(y)) \in F_{M}^{\partial} .
$$

E, sendo $E \in \mathcal{E}$ completamente arbitrário, disso concluímos que $f$ é bornológica.

OBSERVAÇÃo 4.4.4. Tendo em vista o exemplo 4.4.3-d), é imediato ver que, se $(X, d)$ e $(Y, \partial)$ são espaços pseudométricos, e $f: X \longrightarrow Y$ é uma função bornológica, então diam ${ }_{\partial}(f(A))$ é finito para todo $A \subseteq X$ com diâmetro finito em relação a $d$.

O próximo resultado nos mostra que, a exemplo da continuidade uniforme, a bornologia também é preservada pela composição.

Proposição 4.4.5. A composição de funções bornológicas é bornológica. 
Demonstração. Dados espaços coarse $(X, \mathcal{E}),(Y, \mathcal{F})$ e $(Z, \mathcal{G})$, e funções bornológicas

$$
f: X \longrightarrow Y \quad \text { e } g: Y \longrightarrow Z
$$

vamos mostrar que $g \circ f: X \longrightarrow Z$ é bornológica.

Seja $E \in \mathcal{E}$ dado. Como $f$ é bornológica, podemos fixar $F \in \mathcal{F}$ tal que $(f(x), f(y)) \in F$ para todo $(x, y) \in E$. Por outro lado, como $g$ é bornológica, podemos, agora, tomar $G \in \mathcal{G}$ de modo que $(g(u), g(v)) \in G$ para todo $(u, v) \in F$. Disso resulta que, para todo $(x, y) \in X \times X$,

$$
(x, y) \in E \Rightarrow(f(x), f(y)) \in F \Rightarrow(g(f(x)), g(f(y))) \in G \Rightarrow((g \circ f)(x),(g \circ f)(y)) \in G .
$$

E, como $E \in \mathcal{E}$ é arbitrário, isso mostra que $g \circ f$ é bornológica.

O teorema a seguir relaciona continuidade uniforme e bornologia para funções de um espaço vetorial normado em um espaço pseudométrico. Ele será particularmente útil no capítulo 5, quando estivermos trabalhando com funções entre espaços de Banach.

TeOrema 4.4.6. Sejam $\left(X,\|\cdot\|_{X}\right)$ um espaço vetorial normado e $(Y, \partial)$ um espaço pseudométrico. Nessas condições, toda função $f: X \longrightarrow Y$ uniformemente continua é bornológica. ${ }^{5}$

Demonstração. Seja $f: X \longrightarrow Y$ uma função uniformemente contínua. Pelo que vimos no exemplo 4.4.3-d), para concluírmos que $f$ é bornológica, basta mostrarmos que, para cada $\Delta>0$, existe $M>0$ tal que $\partial(f(x), f(y))<M$ para todos $x, y \in X$ tais que $\|x-y\|_{X}<\Delta$. É o que faremos a seguir.

Seja $\Delta>0$ dado. Como $f$ é uniformemente contínua, podemos fixar $\delta>0$ de modo que $\partial\left(f\left(x^{\prime}\right), f\left(y^{\prime}\right)\right)<1$ para todos $x^{\prime}, y^{\prime} \in X$ tais que $\left\|x^{\prime}-y^{\prime}\right\|_{X}<\delta$. Tomemos $M \in \mathbb{N}$ tal que $M>\frac{\Delta}{\delta}$. Vamos mostrar que $\partial(f(x), f(y))<M$ para quaisquer $x, y \in X$ tais que $\|x-y\|_{X}<\Delta$.

Sejam $x$ e $y$ dois pontos quaisquer de $X$ tais que $\|x-y\|_{X}<\Delta$. Para cada $i \in\{0, \ldots, M\}$, seja $z_{i}:=y+\frac{i}{M}(x-y)$. Note que, para todo $i \in\{0, \ldots, M-1\}$,

$$
\left\|z_{i+1}-z_{i}\right\|_{X}=\frac{1}{M}\|x-y\|_{X}<\frac{\Delta}{M}<\delta \Rightarrow \partial\left(f\left(z_{i+1}\right), f\left(z_{i}\right)\right)<1 .
$$

Disso segue que

$$
\partial(f(x), f(y)) \leq \sum_{i=0}^{M-1} \underbrace{\partial\left(f\left(z_{i+1}\right), f\left(z_{i}\right)\right)}_{<1}<\sum_{i=0}^{M-1} 1=M,
$$

como queríamos.

OBSERVAÇÃo 4.4.7. Observe que não vale um resultado análogo ao teorema 4.4 .6 no caso em que $(X, d)$ é apenas um espaço pseudométrico. Com efeito, se tomarmos $X=Y=\mathbb{R}$ e considerarmos $d$ e $\partial$ como sendo, respectivamente, as métricas discreta e usual sobre $\mathbb{R}$, a função

$$
\text { id }: \mathbb{R} \text { } \begin{aligned}
\longrightarrow \mathbb{R} \\
x \longmapsto x
\end{aligned}
$$

\footnotetext{
${ }^{5}$ Em relação às respectivas uniformidades e estruturas coarse limitadas induzidas por $\|\cdot\|_{X}$ e $\partial$.
} 
será uniformemente contínua (veja o exemplo 3.4.5-b)) e, no entanto, não será bornológica, uma vez que, nesse caso, $d(x, y)<2$ quaisquer que sejam $x, y \in \mathbb{R}$ e, para todo $M>0$,

$$
|\operatorname{id}(M+1)-\operatorname{id}(0)|=M+1>M .
$$

Antes de seguirmos para a próxima definição, vale a pena mostrarmos uma última proposição, a qual nos fornece uma caracterização das funções bornológicas. Observe que essa caracterização nada mais é do que uma definição alternativa para bornologia.

Proposição 4.4.8. Uma função $f: X \longrightarrow Y$ é bornológica se, e somente se,

$$
E^{f}:=\{(f(x), f(y)):(x, y) \in E\}
$$

pertence a $\mathcal{F}$ para todo $E \in \mathcal{E}$.

Demonstração. $(\Rightarrow)$ Seja $E \in \mathcal{E}$ dado. Como $f$ é bornológica, podemos fixar $F \in \mathcal{F}$ de modo que $(f(x), f(y)) \in F$ para todo $(x, y) \in E$. Vamos mostrar que $E^{f} \subseteq F$. Para isso, basta notar que, se $(u, v) \in E^{f}$, então existe $(x, y) \in E$ tal que $u:=f(x)$ e $v:=f(y)$. E

$$
(x, y) \in E \Rightarrow(f(x), f(y)) \in F \Rightarrow(u, v) \in F .
$$

Por fim, como $F \in \mathcal{F}$ e $E^{f} \subseteq F$, disso concluímos que $E^{f}$ também pertence a $\mathcal{F}$.

$(\Leftarrow)$ Note que, para cada $E \in \mathcal{E}$, temos, por hipótese, que $E^{f} \in \mathcal{F}$ e que $(f(x), f(y)) \in E^{f}$ para todo $(x, y) \in E$. Isso mostra que $f$ é bornológica.

Definição 4.4 .9 (EXPansividade). Dizemos que uma função $f: X \longrightarrow Y$ é expansível em relação às estruturas coarse $\mathcal{E}$ e $\mathcal{F}$ se, para cada $F \in \mathcal{F}$, existir $E \in \mathcal{E}$ tal que, para todo $(x, y) \in X \times X$

$$
(x, y) \notin E \Rightarrow(f(x), f(y)) \notin F .
$$

Também neste caso, omitiremos as estruturas coarse quando estas estiverem claras pelo contexto.

Considerações análogas às feitas na observação 4.4.2 para as funções bornológicas também valem no caso das funções expansíveis. Elas estão sintetizadas na observação 4.4.10.

ObSERVAÇÃo 4.4.10. Sejam $\mathscr{B}$ e $\mathscr{C}$ bases de $\mathcal{E}$ e de $\mathcal{F}$, respectivamente, e $f: X \longrightarrow Y$ uma função qualquer. São equivalentes:

a) $f$ é expansível;

b) Para todo $C \in \mathscr{C}$, existe $E \in \mathcal{E}$ tal que $(f(x), f(y)) \notin C$ para todos $x, y \in X$ tais que $(x, y) \notin E$;

c) Para todo $F \in \mathcal{F}$, existe $B \in \mathscr{B}$ tal que $(f(x), f(y)) \notin F$ para todos $x, y \in X$ tais que $(x, y) \notin B$.

\section{EXEMPLOS 4.4.11.}

a) Se $\mathcal{E}=\mathcal{P}(X \times X)$, toda função $f: X \longrightarrow Y$ é expansível.

b) Se $\mathcal{F}=\mathcal{P}(\Delta(Y))$, toda função injetora $f: X \longrightarrow Y$ é expansível. Isso segue do fato de que $\Delta(X) \in \mathcal{E}$ e, para cada $F \in \mathcal{F}$ e cada $(x, y) \in X \times X$,

$$
(x, y) \notin \Delta(X) \Rightarrow x \neq y \Rightarrow f(x) \neq f(y) \Rightarrow(f(x), f(y)) \notin \Delta(Y) \Rightarrow(f(x), f(y)) \notin F .
$$


c) No caso em que $\mathcal{E}$ e $\mathcal{F}$ são induzidas por pseudométricas $d$ e $\partial$, respectivamente, pode-se mostrar que uma função $f: X \longrightarrow Y$ é expansível se, e somente se, para todo $M>0$, existe $\Delta>0$ tal que $\partial(f(x), f(y)) \geq M$ para todos $x, y \in X$ tais que $d(x, y) \geq \Delta$. A demonstração disso é análoga à que foi feita no item d) do exemplo 4.4.2 e decorre diretamente da observação 4.4.10.

Observe que a composição de funções expansíveis também é expansível. Dada a sua importância, enunciaremos esse resultado na forma de uma proposição. Sua demonstração é análoga à da proposição 4.4.5, e fica a cargo do leitor.

Proposição 4.4.12. A composição de funções expansíveis é expansível.

À semelhança do que foi feito para as funções uniformemente contínuas e bornológicas, apresentaremos, agora, uma caracterização das funções expansíveis.

Proposição 4.4.13. Uma função $f: X \longrightarrow Y$ é expansível se, e somente se,

$$
F_{f}:=\{(x, y) \in X \times X:(f(x), f(y)) \in F\}
$$

pertence a $\mathcal{E}$ para cada $F \in \mathcal{F}$.

Demonstração. $(\Rightarrow)$ Seja $F \in \mathcal{F}$ dado. Como $f$ é expansível, podemos tomar $E \in \mathcal{E}$ de modo que, para todo $(x, y) \in X \times X$,

$$
(x, y) \notin E \Rightarrow(f(x), f(y)) \notin F .
$$

Logo, para todo $(x, y) \in X \times X$,

$$
(x, y) \in F_{f} \Rightarrow(f(x), f(y)) \in F \Rightarrow(x, y) \in E .
$$

Disso resulta que $F_{f} \subseteq E$.

Por fim, note que

$$
\left(E \in \mathcal{E} \text { e } F_{f} \subseteq E\right) \Rightarrow F_{f} \in \mathcal{E}
$$

como queríamos.

$(\Leftarrow)$ Observe que, para cada $F \in \mathcal{F}$, temos, por hipótese, que $F_{f} \in \mathcal{E}$. Além disso, segue da definição de $F_{f}$ que, para todo $(x, y) \in X \times X$,

$$
(x, y) \notin F_{f} \Rightarrow(f(x), f(y)) \notin F,
$$

o que, por sua vez, mostra que $f$ é expansível.

Sejam $(M, d)$ um espaço métrico, $A$ um conjunto não vazio e

$$
L(A, M):=\left\{f: A \longrightarrow M: \operatorname{diam}_{d}(\operatorname{Im}(f))<+\infty\right\} .
$$

Nessas condições, não é difícil mostrar que a função $\partial: L(A, M) \times L(A, M) \longrightarrow \mathbb{R}$ tal que

$$
\partial(f, g)=\sup \{d(f(t), g(t)): t \in A\}
$$

está bem definida e é uma métrica sobre $L(A, M)$. De modo geral, não podemos estender tal métrica ao conjunto de todas as funções de $A$ em $M$. Apesar disso, mesmo no caso em que as imagens de $f, g: A \longrightarrow M$ não possuem diâmetro finito, é possível estabelecer uma noção 
(grosseira) de proximidade entre entre essas duas funções. Uma possibilidade, nesse sentido, é dizer que $f$ e $g$ estão próximas em $(M, d)$ se

$$
\sup \{d(f(t), g(t)): t \in A\}
$$

for finito. Observe que isso equivale a dizer que $f$ e $g$ estão próximas em $(M, d)$ se, e somente se, existe $\alpha>0$ tal que

$$
(f(t), g(t)) \in E_{\alpha}^{d} \quad \forall t \in A .
$$

E, por sua vez, essa última observação nos sugere uma forma natural de estender a noção de proximidade a funções cujo contradomínio é um espaço coarse arbitrário. Mais especificamente, podemos formular a seguinte definição:

Definição 4.4.14. Dado um conjunto não vazio $S$, dizemos que as funções $f, g: S \longrightarrow X$ estão próximas em $(X, \mathcal{E})$ se existir $E \in \mathcal{E}$ tal que $(f(s), g(s)) \in E$ para todo $s \in S$.

OBSERVAÇÃo 4.4.15. Dados $S, f$ e $g$ como na definição 4.4.14, é imediato ver que $f$ e $g$ estarão próximas em $(X, \mathcal{E})$ se, e somente se,

$$
\{(f(s), g(s)): s \in S\} \in \mathcal{E} .
$$

Dado um conjunto não vazio $S$, não é difícil mostrar que a relação $\underset{\text { prox }}{\sim}$, definida sobre a classe de todos os pares de funções de $S$ em $X$ de modo que $f \underset{\text { prox }}{\sim} g$ se, e somente se, $f$ e $g$ estão próximas em $(X, \mathcal{E})$, é uma relação de equivalência sobre a coleção $\mathscr{F}(S, X)$ das funções de $S$ em $X$.

Para constatar isso, inicialmente observe que $f \underset{\text { prox }}{\sim} f$ qualquer que seja $f \in \mathscr{F}(S, X)$, uma vez que, para toda $f \in \mathscr{F}(S, X)$, temos que

$$
(f(s), f(s)) \in \Delta(X) \quad \forall s \in S .
$$

Suponhamos, agora, que $f, g, h \in \mathscr{F}(S, X)$ sejam tais que $f \underset{\text { prox }}{\sim} g$ e $g \underset{\text { prox }}{\sim} h$, e vamos mostrar que $g \underset{\text { prox }}{\sim} f$ e que $f \underset{\text { prox }}{\sim} h$.

Com efeito, dado que, por hipótese, $f \underset{\text { prox }}{\sim} g$ e $f \underset{\text { prox }}{\sim} h$, podemos fixar $E, F \in \mathcal{E}$ de modo que

$$
(f(s), g(s)) \in E \text { e }(f(s), h(s)) \in F
$$

para todo $s \in S$.

Observe, em particular, que $E^{-1}, F \circ E \in \mathcal{E}$, uma vez que $E$ e $F$ são, eles próprios, elementos de $\mathcal{E}$. Além disso, é imediato ver que, para todo $s \in S$,

$$
[(f(s), g(s)) \in E \mathrm{e}(g(s), h(s)) \in F] \Rightarrow(f(s), h(s)) \in F \circ E,
$$

$\mathrm{e}$

$$
(f(s), g(s)) \in E \Leftrightarrow(g(s), f(s)) \in E^{-1},
$$

a partir do que concluímos que $g \underset{\text { prox }}{\sim} f$ e que $f \underset{\text { prox }}{\sim} h$. E, uma vez que $f, g, h \in \mathscr{F}(S, X)$ são completamente arbitrárias, disso resulta que, além de reflexiva, $\sim$ prox é, também, simétrica e transitiva, sendo, portanto, uma relação de equivalência.

O próximo passo, agora, é verificar se essa é, de fato, uma noção adequada para descrever a proximidade de funções entre espaços coarse, em que, com o termo "adequada", queremos 
dizer, simplesmente, que duas funções, $f, g: X \longrightarrow Y$, as quais estão próximas em $(Y, \mathcal{F})$, devem possuir as mesmas propriedades no que se refere à geometria de larga escala. Para isso, precisamos, inicialmente, determinar quais propriedades de funções entre espaços coarse são conservadas pela relação de proximidade. Vamos mostrar, a seguir, que este é caso da bornologia e da expansividade.

Proposição 4.4.16. Sejam $f, g: X \longrightarrow Y$ duas funções próximas em $(Y, \mathcal{F})$. Nessas condiçôes:

a) f é bornológica se, e somente se, g for bornológica;

b) $f$ é expansível se, e somente se, $g$ for expansível.

Demonstração. Antes de mais nada, fixemos $G \in \mathcal{F}$ de modo que $(f(x), g(x)) \in G$ para todo $x \in X{ }^{6}$

a) Suponhamos inicialmente, que $f$ seja bornológica, e vamos mostrar que, nesse caso, $g$ é bornológica. Feito isso, bastará invertermos os papéis de $f$ e de $g$ e repetirmos o argumento para obtermos a outra implicação.

Para isso, tomemos $E \in \mathcal{E}$ de modo arbitrário. Como $E \in \mathcal{E}$ e $f$ é bornológica, segue da proposição 4.4 .8 que $E^{f} \in \mathcal{F}$. E, por sua vez,

$$
G, E^{f} \in \mathcal{F} \Rightarrow G \circ E^{f} \circ G^{-1} \in \mathcal{F}
$$

Vamos mostrar que $(g(x), g(y)) \in G \circ E^{f} \circ G^{-1}$ para todo $(x, y) \in E$. Com efeito, dados $x, y \in X$, é fácil ver que

$$
\begin{aligned}
(x, y) \in E & \Rightarrow\left[(f(x), f(y)) \in E^{f} \mathrm{e}(f(x), g(x)),(f(y), g(y)) \in G\right] \\
& \Rightarrow\left[(g(x), f(x)) \in G^{-1},(f(x), f(y)) \in E^{f} \mathrm{e}(f(y), g(y)) \in G\right] \\
& \Rightarrow\left[(g(x), f(y)) \in E^{f} \circ G^{-1} \mathrm{e}(f(y), g(y)) \in G\right] \Rightarrow(g(x), g(y)) \in G \circ E^{f} \circ G^{-1} .
\end{aligned}
$$

E, sendo $E \in \mathcal{E}$ um elemento qualquer, disso concluímos que $g$ é bornológica.

b) Suponhamos, agora, que $f$ seja expansível, e vamos mostrar que disso segue que $g$ é expansível. Para tanto, inicialmente fixemos $F \in \mathcal{F}$ de modo arbitrário. Observe que

$$
F, G \in \mathcal{F} \Rightarrow G^{-1} \circ F \circ G \in \mathcal{F} .
$$

Como $f$ é expansível, disso resulta que $\left(G^{-1} \circ F \circ G\right)_{f} \in \mathcal{E}$ (veja a proposição 4.4.13). Além disso, dados $x, y \in X$, é fácil ver que

$$
\begin{aligned}
{[(g(x), g(y)) \in F \text { e }(f(x), g(x)),(f(y), g(y)) \in G] } & \Rightarrow(f(x), f(y)) \in G^{-1} \circ F \circ G \\
& \Rightarrow(x, y) \in\left(G^{-1} \circ F \circ G\right)_{f} .
\end{aligned}
$$

E, uma vez que, por construção, $(f(z), g(z)) \in G$ para todo $z \in X$, disso resulta que

$$
(x, y) \notin\left(G^{-1} \circ F \circ G\right)_{f} \Rightarrow(g(x), g(y)) \notin F,
$$

\footnotetext{
${ }^{6} \mathrm{Um}$ tal $G$ certamente existe, dado que, por hipótese, $f$ e $g$ estão próximas em $(Y, \mathcal{F})$.
} 
a partir do que concluímos que $g$ é expansível.

Analogamente, se $g$ for expansível, basta repetirmos o argumento anterior substituindo $f$ por $g$ e $g$ por $f$ para concluírmos que $f$ é expansível.

Explorando a relação de proximidade, podemos, também, mostrar que bornologia e expansividade estão, na verdade, intimamente relacionadas.

Proposição 4.4.17. Sejam $f: X \longrightarrow Y$ e $g: Y \longrightarrow X$ funções tais que $g \circ f$ está próxima de $\operatorname{id}_{X}$ em $(X, \mathcal{E})$. Nessas condições:

(1) Se g for bornológica, então f será expansivel;

(2) Se g for expansível, então $f$ será bornológica e $f \circ g$ estará próxima de $\mathrm{id}_{Y}$ em $(Y, \mathcal{F})$.

Demonstração. Para cada $x \in X$, definamos

$$
a_{x}:=(g \circ f)(x)=g(f(x)) .^{7}
$$

Em seguida, fixemos $D \in \mathcal{E}$ tal que $\left(x, a_{x}\right)=(x,(g \circ f)(x)) \in D$ para todo $x \in X{ }^{8}$

(1) Suponhamos, inicialmente, que $g$ seja bornológica, e mostremos que, nesse caso, $f$ é expansível. Para tanto, precisamos mostrar que, para cada $F \in \mathcal{F}$, existe $E \in \mathcal{E}$ tal que $(f(x), f(y)) \notin F$ para todo $(x, y) \in(X \times X) \backslash E$.

Seja $F \in \mathcal{F}$ dado. Como, por hipótese, $g$ é bornológica, segue da proposição 4.4 .8 que $F^{g} \in \mathcal{E}$. E, uma vez que $D, F^{g} \in \mathcal{E}$, temos também que $D^{-1} \circ F^{g} \circ D \in \mathcal{E}$.

Vamos mostrar que, para cada $(x, y) \in X \times X$,

$$
(f(x), f(y)) \in F \Rightarrow(x, y) \in D^{-1} \circ F^{g} \circ D .
$$

Como $F \in \mathcal{F}$ é arbitrário, disso seguirá (pela contrapositiva) que $f$ é expansível.

Para isso, é suficiente notar que, dados $x, y \in X$, segue da definição de $F^{g}$ que

$$
(f(x), f(y)) \in F \Rightarrow(g(f(x)), g(f(y))) \in F^{g} \Rightarrow\left(a_{x}, a_{y}\right) \in F^{g} .
$$

E, por sua vez,

$$
\begin{aligned}
{\left[\left(a_{x}, a_{y}\right) \in F^{g} \mathrm{e}\left(x, a_{x}\right),\left(y, a_{y}\right) \in D\right] } & \Rightarrow\left[\left(x, a_{x}\right) \in D,\left(a_{x}, a_{y}\right) \in F^{g} \mathrm{e}\left(a_{y}, y\right) \in D^{-1}\right] \\
& \Rightarrow\left[\left(x, a_{y}\right) \in F^{g} \circ D \mathrm{e}\left(a_{y}, y\right) \in D^{-1}\right] \\
& \Rightarrow(x, y) \in D^{-1} \circ F^{g} \circ D .
\end{aligned}
$$

(2) Suponhamos, agora, que $g$ seja expansível, e vamos mostrar como, a partir disso, podemos concluir que $f$ é bornológica e que $f \circ g$ está próxima de $\operatorname{id}_{Y}$.

Para tanto, vamos começar fixando $E \in \mathcal{E}$ de modo arbitrário. Observe que

$$
D, E \in \mathcal{E} \Rightarrow D \circ E \circ D^{-1} \in \mathcal{E}
$$

Como $g$ é expansível, disso resulta que $\left(D \circ E \circ D^{-1}\right)_{g} \in \mathcal{F}$ (veja a proposição 4.4.13).

\footnotetext{
${ }^{7}$ Embora não essencial, o uso dessa notação tornará a demonstração muito mais compacta e organizada.

${ }^{8} \mathrm{Um}$ tal $D$ certamente existe, uma vez que, por hipótese, $g \circ f$ está próxima de $\mathrm{id}_{X}$.
} 
A seguir, vamos mostrar que $(f(x), f(y)) \in\left(D \circ E \circ D^{-1}\right)_{g}$ para todo $(x, y) \in E$. Como $E \in \mathcal{E}$ é arbitrário, disso seguirá que $f$ é bornológica. Com efeito, dados $x, y \in X$, não é difícil notar que

$$
\begin{aligned}
{\left[(x, y) \in E \text { e }\left(x, a_{x}\right),\left(y, a_{y}\right) \in D\right] } & \Rightarrow\left[\left(a_{x}, x\right) \in D^{-1} \mathrm{e}\left(x, a_{y}\right) \in D \circ E\right] \\
& \Rightarrow\left(a_{x}, a_{y}\right) \in D \circ E \circ D^{-1} \\
& \Rightarrow(g(f(x)), g(f(y))) \in D \circ E \circ D^{-1} \\
& \Rightarrow(f(x), f(y)) \in\left(D \circ E \circ D^{-1}\right)_{g} .
\end{aligned}
$$

Por fim, precisamos, ainda, mostrar que $f \circ g$ está próxima de $i_{Y}$. Para isso, inicialmente observe que, sendo $g$ uma função expansível e $D$ um elemento de $\mathcal{E}$, segue da proposição 4.4 .13 que $D_{g} \in \mathcal{F}$. Além disso, dado $y \in Y$, é fácil ver que

$$
\left(g(y), a_{g(y)}\right) \in D \Rightarrow(g(y), g(f(g(y)))) \in D \Rightarrow(y, f(g(y))) \in D_{g} \Rightarrow(y,(f \circ g)(y)) \in D_{g} .
$$

E como, por hipótese, $\left(g(y), a_{g(y)}\right) \in D$ para todo $y \in Y$, disso concluímos que $f \circ g$ está próxima de $\operatorname{id}_{Y}$.

A proposição 4.4.17 tem um importante corolário, o qual diz respeito às funções bijetoras entre espaços coarse.

Corolário 4.4.18. Uma função bijetora $f: X \longrightarrow Y$ é bornológica se, e somente se, $f^{-1}$ é expansivel.

Comparando as proposições 3.4 .7 e 4.4.8, fica claro que, do mesmo como os morfismos entre espaços uniformes são as funções uniformemente contínuas, as funções bornológicas são os morfismos entre espaços coarse (conforme antecipamos no início da seção). Tendo isso em vista, seria razoável definirmos um "isomorfismo grosseiro" como sendo uma função bornológica, bijetora, e com inversa bornológica, da mesma maneira como, no capítulo 3, definimos um isomorfismo uniforme como sendo uma função uniformemente contínua, bijetora, e com inversa uniformemente contínua. Essa definição, no entanto, é muito restritiva. Ela exige, em particular, que dois espaços "grosseiramente isomorfos" (em relação a um par qualquer de estruturas coarse) possuam a mesma cardinalidade. E a presença um tal empecilho, por sua vez, faz com que essa não seja a noção adequada para formalizar, por exemplo, a nossa ideia intuitiva de que $\mathbb{Z}$ e $\mathbb{R}$ são os mesmos de um ponto de vista de larga escala. Apesar disso, seria desejável obter algum tipo de aplicação entre espaços coarse a qual nos permitisse "transportar" as propriedades da geometria de larga escala de um para o outro, e vice-versa. Em outras palavras, gostaríamos de obter funções entre espaços coarse as quais nos permitissem determinar quando dois espaços coarse são "equivalentes" de um ponto de vista da geometria de larga escala, mas sem que, para isso, fosse necessário considerar apenas funções bijetoras. Uma alternativa, nesse caso, é recorrer à noção de proximidade definida em 4.4.14. Isto é: ao invés de exigirmos que existam funções bornológicas $f: X \longrightarrow Y$ e $g: Y \longrightarrow X$ tais que $f \circ g=\mathrm{id}_{Y}$ e $g \circ f=\mathrm{id}_{X}, \operatorname{vamos}$ apenas pedir que existam funções bornológicas $f: X \longrightarrow Y$ e $g: Y \longrightarrow X$ tais que $f \circ g$ e $g \circ f$ estejam próximas, respectivamente, $\mathrm{de} \mathrm{id}_{Y}$ e $\mathrm{de}_{\mathrm{id}}$. Isso nos leva à seguinte definição:

Definição 4.4.19 (EquivalênCia Grosseira). Dizemos que uma função $f: X \longrightarrow Y$ é uma equivalência grosseira em relação a $\mathcal{E}$ e $\mathcal{F}$ se for bornológica e existir uma função bornológica $g: Y \longrightarrow X$ tal que $f \circ g$ e $g \circ f$ estejam próximas, respectivamente, de $\mathrm{id}_{Y}$ e de $\operatorname{id}_{X}$. 
Se existir uma equivalência grosseira entre $(X, \mathcal{E})$ e $(Y, \mathcal{F})$, diremos que $(X, \mathcal{E})$ e $(Y, \mathcal{F})$ são grosseiramente equivalentes.

OBSERVAÇÃO 4.4.20.

a) A função id : $X \longrightarrow X$ tal que id $(x)=x$ é uma equivalência grosseira em relação a qualquer estrutura coarse sobre $X$. Isso mostra que todo espaço coarse é grosseiramente equivalente a si mesmo.

b) Observe que a definição 4.4.19 é simétrica em relação a $(X, \mathcal{E})$ e $(Y, \mathcal{F})$. Disso concluímos que $(X, \mathcal{E})$ será grosseiramente equivalente a $(Y, \mathcal{F})$ se, e somente se, $(Y, \mathcal{F})$ for grosseiramente equivalente a $(X, \mathcal{E})$.

c) Mostraremos, mais adiante, que a composição de equivalências grosseiras é, também, uma equivalência grosseira. Tendo em vista as observações a) e b), disso seguirá que, à noção de equivalência grosseira definida em 4.4 .19 corresponde, de fato, uma relação de equivalência sobre a classe dos espaços coarse, o que, por sua vez, justifica o uso do termo equivalência para designar esse tipo de aplicação entre espaços coarse.

O conceito de equivalência grosseira, tal como definido em 4.4.19, induz, naturalmente, uma noção de "mergulho grosseiro".

Definição 4.4.21 (Mergulho Grosseiro). Dizemos que uma função $f: X \longrightarrow Y$ é um mergulho grosseiro em relação a $\mathcal{E}$ e $\mathcal{F}$ se a função $f^{\prime}: X \longrightarrow \operatorname{Im}(f)$ tal que $f^{\prime}(x)=f(x)$ para todo $x \in X$ for uma equivalência grosseira em relação a $\mathcal{E}$ e $\mathcal{F}_{\operatorname{Im}(f)}$.

A proposição a seguir é uma consequência imediata da definição 4.4.21.

Proposição 4.4.22. Todo mergulho grosseiro sobrejetor $f: X \longrightarrow Y$ é uma equivalência grosseira.

Embora, em termos teóricos, a definição 4.4.19 reflita bem a nossa intuição, na prática pode ser muito difícil encontrar um par $(f, g)$ de funções satisfazendo as hipóteses de 4.4.19. Por essa razão, vamos enunciar, a seguir, uma caracterização muito útil para as equivalências grosseiras, a qual nos permitirá considerar, em nossas análises, uma única função (ao invés de um par). Para isso, no entanto, precisaremos, inicialmente, fazer algumas definições preliminares.

Definição 4.4.23 (Conjunto COlimitado). Um conjunto $A \subseteq Y$ é dito colimitado em $(Y, \mathcal{F})$ se existir algum $F \in \mathcal{F}$ tal que $F^{-1}[A]=Y$.

Note que, pela definição 4.4.23, um subconjunto $A$ de $Y$ será colimitado em $(Y, \mathcal{F})$ se, e somente se, existir $F \in \mathcal{F}$ tal que, para todo $y \in Y$, exista $a \in A$ tal que $(y, a) \in F$.

OBSERVAÇÃo 4.4.24. Nos itens b) e c), a seguir, consideremos um conjunto arbitrário $A \subseteq Y$.

a) Note que $Y$ é colimitado em $(Y, \mathcal{F})\left(\right.$ pois $\Delta(Y) \in \mathcal{F}$ e $\left.[\Delta(Y)]^{-1}[Y]=Y\right)$.

b) Suponhamos que $\mathcal{F}$ seja induzida por uma pseudométrica $\partial$ sobre $Y$. Como, nesse caso, $\mathscr{C}:=\left\{F_{M}^{\partial}: M>0\right\}$ é base de $\mathcal{F}$, não é difícil mostrar que $A$ será colimitado em $(Y, \mathcal{F})$ se, e somente se, existir $M>0$ tal que, para cada $y \in Y$, exista $a \in A$ tal que $\partial(y, a)<M$.

Além disso, é imediato ver que, nessas condições, todo subconjunto de $Y$ denso em $\left(Y, \tau_{\partial}\right)$ é, trivialmente, colimitado. Com efeito, dado um conjunto $D \subseteq Y$ denso em $\left(Y, \tau_{\partial}\right)$, segue diretamente de 2.7 .9 e da definição de fecho que, para cada $y \in Y$, podemos fixar $d \in D$ de modo que $\partial(y, d)<1$. 
c) Com frequência, estaremos interessados no caso em que $A=\operatorname{Im}(f)$, em que $f$ é uma função de $X$ em $Y$. Observe que, se $f$ for sobrejetora, $\operatorname{Im}(f)$ será, trivialmente, colimitada em $(Y, \mathcal{F})$. Isso decorre diretamente do item a), uma vez que, nesse caso, teremos $\operatorname{Im}(f)=Y$.

O item c) da observação 4.4.24 motiva a seguinte definição adicional:

Definição 4.4.25. Dizemos que uma função $f: X \longrightarrow Y$ é colimitada em $(Y, \mathcal{F})$ se $\operatorname{Im}(f)$ for colimitada em $(Y, \mathcal{F})$.

Vejamos, agora, um exemplo importante de conjunto colimitado, a partir do qual será possível perceber mais claramente as ideias por trás da definição 4.4.23.

Exemplo 4.4.26. O conjunto dos números inteiros, $\mathbb{Z}$, é colimitado em $\left(\mathbb{R}, \mathcal{E}_{d_{|\cdot|}}\right)$, pois, para cada $x \in \mathbb{R}, n:=\max \{j \in \mathbb{Z}: j \leq x\}$ é um inteiro tal que $|x-n|<1$.

A figura 4.3 ilustra o comentário anterior.

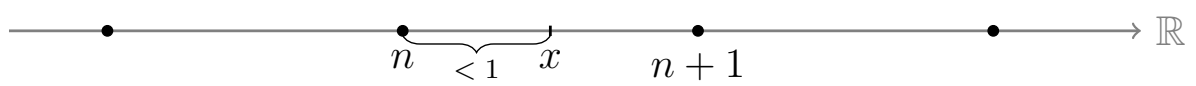

Figura 4.3: Na figura, $n$ é o maior inteiro menor ou igual a $x$.

Por um raciocício análogo, podemos mostrar também que, para todo $n \in \mathbb{N}, \mathbb{Z}^{n}$ é colimitado em $\left(\mathbb{R}^{n}, \mathcal{E}_{d_{\|\cdot\|}}\right)$. Para isso, fixemos $n \in \mathbb{N}$ e $\left(x_{1}, \ldots, x_{n}\right) \in \mathbb{R}^{n}$ de modo arbitrário, e, para cada $k \in\{1, \ldots, n\}$, tomemos $m_{k}:=\max \left\{j \in \mathbb{Z}: j \leq x_{k}\right\}$. Se assim o fizermos, teremos que, para cada $k \in\{1, \ldots, n\},\left|x_{k}-m_{k}\right|<1$, a partir do que concluímos que

$$
\left\|\left(x_{1}, \ldots, x_{n}\right)-\left(m_{1}, \ldots, m_{n}\right)\right\|=\left\|\left(x_{1}-m_{1}, \ldots, x_{n}-m_{n}\right)\right\| \leq \sum_{k=1}^{n} \underbrace{\left|x_{k}-m_{k}\right|}_{<1}<n .
$$

E, sendo $\left(x_{1}, \ldots, x_{n}\right) \in \mathbb{R}^{n}$ um ponto qualquer, disso segue o resultado.

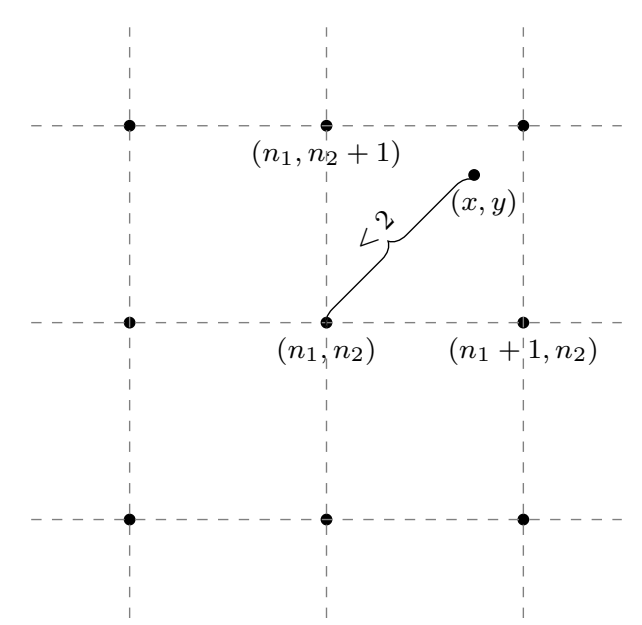

Figura 4.4: Ilustração do processo no caso em que $n=2$.

ObSERVAÇÃo 4.4.27. Dado $n \in \mathbb{N}$, definamos $\iota_{n}: \mathbb{Z}^{n} \longrightarrow \mathbb{R}^{n}$ e $\varphi_{n}: \mathbb{R}^{n} \longrightarrow \mathbb{Z}^{n}$ de modo que

$$
\iota_{n}\left(a_{1}, \ldots, a_{n}\right)=\left(a_{1}, \ldots, a_{n}\right)
$$

$\mathrm{e}$

$$
\varphi_{n}\left(x_{1}, \ldots, x_{n}\right)=\left(m_{1}, \ldots, m_{n}\right),
$$


em que, para cada $k \in\{1, \ldots, n\}, m_{k}:=\max \left\{j \in \mathbb{Z}: j \leq x_{k}\right\}$. Tendo em mente o exemplo anterior, é imediato ver que, para cada $\left(x_{1}, \ldots x_{n}\right) \in \mathbb{R}^{n}$,

$$
\begin{aligned}
\left\|\left(x_{1}, \ldots, x_{n}\right)-\left(\iota_{n} \circ \varphi_{n}\right)\left(x_{1}, \ldots, x_{n}\right)\right\| & =\left\|\left(x_{1}, \ldots, x_{n}\right)-\iota_{n}\left(m_{1}, \ldots, m_{n}\right)\right\| \\
& =\left\|\left(x_{1}, \ldots, x_{n}\right)-\left(m_{1}, \ldots, m_{n}\right)\right\|<n,
\end{aligned}
$$

a partir do que concluímos que $\iota_{n} \circ \varphi_{n}$ está próxima de $\operatorname{id}_{\mathbb{R}^{n}}$ em $\left(\mathbb{R}^{n}, \mathcal{E}_{d_{\|\cdot\|}}\right)$. Mais do que apenas um fato isolado, isso é consequência de uma relação bastante geral entre funções colimitadas e proximidade, da qual faremos uso na demonstração do próximo resultado.

As proposições a seguir são consequências simples da definição 4.4.23.

Proposição 4.4.28. Sejam $A, B \subseteq X$ tais que $A \subseteq B$. Nessas condições, se A for colimitado em $(X, \mathcal{E})$, então $B$ será, também, colimitado em $(X, \mathcal{E})$.

Demonstração. Com efeito, dado que, por hipótese, $A$ é colimitado em $(X, \mathcal{E})$, podemos fixar $E \in \mathcal{E}$ de modo que $E^{-1}[A]=X$. E, como $A \subseteq B$, disso resulta que

$$
X=E^{-1}[A] \subseteq E^{-1}[B] \subseteq X,
$$

a partir do que concluímos que $E^{-1}[B]=X$.

Proposição 4.4.29. Sejam d uma pseudométrica sobre $X$ e $A \subseteq X$ um subconjunto não vazio. Nessas condições, se $X \backslash A$ possuir diâmetro finito, então A será (trivialmente) colimitado em $\left(X, \mathcal{E}_{d}\right)$.

Demonstração. Isso é óbvio se $X \backslash A=\emptyset$, uma vez que, nesse caso, $A=X$. Sendo assim, podemos supor, deste ponto em diante, que $X \backslash A$ é não vazio.

Sejam $a_{0} \in A$ e $x_{0} \in X \backslash A$ dois pontos quaisquer. Como, por hipótese, $\operatorname{diam}_{d}(A)$ é finito, é imediato ver que a constante

$$
M:=\operatorname{diam}_{d}(A)+d\left(x_{0}, a_{0}\right)
$$

está bem definida. Tomemos, agora, um ponto $y \in X$ de modo arbitrário, e vamos mostrar que existe $a \in A$ tal que $d(y, a)<M$. Com efeito, isso é imediato se $y \in A$, uma vez que, nesse caso, $d(y, y)=0<M$. E, se, ao contrário, $y \in X \backslash A$, é suficiente notar, para tanto, que

$$
d\left(y, a_{0}\right) \leq d\left(y, x_{0}\right)+d\left(x_{0}, a_{0}\right) \leq \operatorname{diam}_{d}(A)+d\left(x_{0}, a_{0}\right)=M .
$$

OBSERVAÇÃo 4.4.30. Note que não vale a recíproca da proposição 4.4.29, uma vez que, pelo exemplo $4.4 .26, \mathbb{Z}$ é colimitado em $\left(\mathbb{R}, \mathcal{E}_{d_{|\cdot|}}\right)$, e, no entanto, $\mathbb{R} \backslash \mathbb{Z}$ não possui diâmetro finito em relação a $d_{|\cdot| \cdot}$.

Após esta pequena digressão, estamos, enfim, em condições de enunciar o próximo teorema.

TeOrema 4.4.31. Uma função $f: X \longrightarrow Y$ é uma equivalência grosseira se, e somente se, for bornológica, expansivel e colimitada em $(Y, \mathcal{F})$.

Demonstração. $(\Rightarrow)$ Dado que, por hipótese, $f$ é uma equivalência grosseira, podemos fixar uma função bornológica $g: Y \longrightarrow X$ de tal modo que $g \circ f$ e $f \circ g$ estejam próximas, respectivamente, de $\operatorname{id}_{X}$ e de $\operatorname{id}_{Y}$.

Como $g$ é bornológica e $g \circ f$ está próxima de $\mathrm{id}_{X}$, segue da proposição 4.4 .17 que $f$ é expansível. Assim sendo, precisamos apenas mostrar que $\operatorname{Im}(f)$ é colimitada em $(Y, \mathcal{F})$. E, por sua vez, isso segue diretamente da hipótese de que $f \circ g$ está próxima de $\mathrm{id}_{Y}$, uma vez que, nesse caso, existe $F \in \mathcal{F}$ tal que $(y, f(g(y))) \in F$ para todo $y \in Y$. 


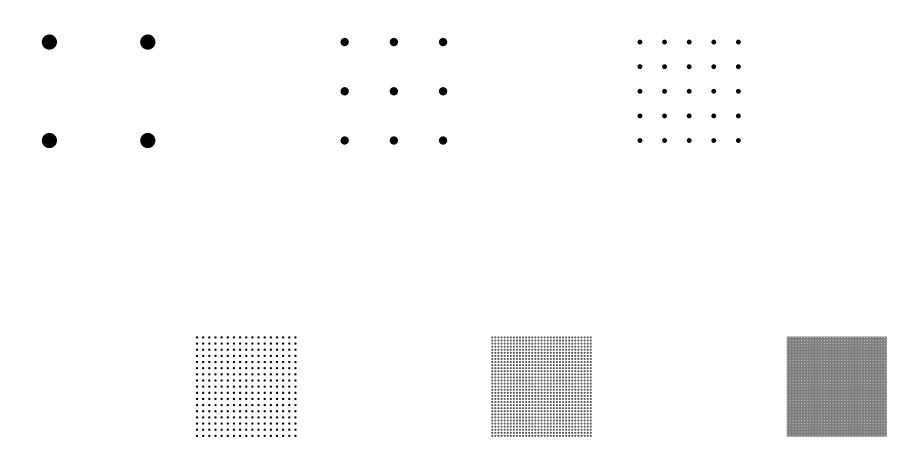

Figura 4.5: A figura acima mostra que $\mathbb{Z}^{2}$ assemelha-se a $\mathbb{R}^{2}$ quando observado a partir de grandes distâncias.

$(\Leftarrow)$ Como $\operatorname{Im}(f)$ é colimitada em $(Y, \mathcal{F})$, podemos tomar $F \in \mathcal{F}$ de tal modo que, para cada $y \in Y$, exista $x \in X$ tal que $(y, f(x)) \in F$. Para cada $y \in Y$, fixemos $x_{y} \in X$ de modo que $\left(y, f\left(x_{y}\right)\right) \in F$. Em seguida, consideremos a função $g: Y \longrightarrow X$ tal que $g(y)=x_{y}$.

Observe que, para todo $y \in Y$,

$$
\left(y, f\left(x_{y}\right)\right) \in F \Rightarrow(y, f(g(y))) \in F \Rightarrow(y,(f \circ g)(y)) \in F .
$$

Isso mostra que $f \circ g$ está próxima de $\operatorname{id}_{Y}$. E, sendo $f$ uma função expansível, disso concluímos, pela proposição 4.4.17, que $g$ é bornológica e que $g \circ f$ está próxima $\mathrm{de}_{\mathrm{i}} \mathrm{d}_{X}$, o que, por sua vez, completa a prova de $f$ é uma equivalência grosseira.

O corolário a seguir, o qual explicita a relação entre as definições 4.4 .25 e 4.4.14, decorre imediatamente das ideias envolvidas na demonstração do teorema 4.4.31.

Corolário 4.4.32. Uma função $f: X \longrightarrow Y$ é colimitada em $(Y, \mathcal{F})$ se, e somente se, existe uma função $g: Y \longrightarrow X$ tal que $f \circ g$ esteja próxima de $\operatorname{id}_{Y}$ em $(Y, \mathcal{F})$.

No que segue, vamos discutir alguns exemplos simples de equivalências grosseiras. Em particular, mostraremos que, para cada $n \in \mathbb{N},\left(\mathbb{Z}^{n}, \mathcal{E}_{d_{\|\cdot\|_{\mathbb{Z}}}}\right)$ é grosseiramente equivalente a $\left(\mathbb{R}, \mathcal{E}_{d_{\|\cdot\|}}\right) .^{9}$ A figura 4.5 ilustra, intuitivamente, esse fato no caso em que $n=2$. Em cada um dos exemplos, utilizaremos a abordagem que se mostrar mais conveniente, seja ela baseada na definição 4.4.19 ou na caracterização 4.4.31.

EXEMPLOS 4.4.33.

a) Para cada $n \in \mathbb{N}$, as funções $\iota_{n}: \mathbb{Z}^{n} \longrightarrow \mathbb{R}^{n}$ e $\varphi_{n}: \mathbb{R}^{n} \longrightarrow \mathbb{Z}^{n}$ tais que

$$
\iota_{n}\left(a_{1}, \ldots, a_{n}\right)=\left(a_{1}, \ldots, a_{n}\right)
$$

$\mathrm{e}$

$$
\varphi_{n}\left(x_{1}, \ldots, x_{n}\right)=\left(m_{1}, \ldots, m_{n}\right),
$$

em que, para cada $k \in\{1, \ldots, n\}, m_{k}:=\max \left\{j \in \mathbb{Z}: j \leq x_{k}\right\}$, são equivalências grosseiras em relação às estruturas coarse limitadas induzidas pelas métricas usuais sobre $\mathbb{Z}^{n}$ e $\mathbb{R}^{n}$.

Demonstração. Com efeito, dado $n \in \mathbb{N}$, segue da observação 4.4 .27 que $\iota_{n} \circ \varphi_{n}$ está próxima

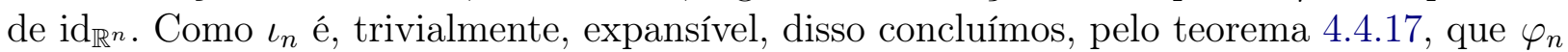
é bornológica e que $\varphi_{n} \circ \iota_{n}$ está próxima de $\operatorname{id}_{\mathbb{Z}^{n}}$. E, uma vez que $\iota_{n}$ é, também, bornológica, isso completa a prova de que $\iota_{n}$ e $\varphi_{n}$ são equivalências grosseiras.

\footnotetext{
${ }^{9}$ Sendo $d_{\|\cdot\|_{\mathbb{Z}}}$ a restrição de $d_{\|\cdot\|}$ a $\mathbb{Z} \times \mathbb{Z}$.
} 
b) Sejam $p \in X$ um ponto qualquer e $\mathcal{E}_{p}:=\{\emptyset,\{(p, p)\}\}$ a única estrutura coarse possível sobre $\{p\}$. Nessas condições, $X \times X \in \mathcal{E}$ se, e somente se,

$$
\begin{aligned}
f: X & \longrightarrow\{p\} \\
x & \longmapsto p
\end{aligned}
$$

for uma equivalência grosseira entre $(X, \mathcal{E})$ e $\left(\{p\}, \mathcal{E}_{p}\right)$.

Demonstração. Inicialmente, observe que $f$ é, trivialmente, sobrejetora, e, portanto, colimitada em $\left(\{p\}, \mathcal{E}_{p}\right)$. Também não é difícil ver que $f$ é bornológica, uma vez que, por definição, $\{(p, p)\} \in \mathcal{E}_{p}$ e $(f(x), f(y)) \in\{(p, p)\}$ para todos $x, y \in X$. E, assim sendo, é suficiente mostrarmos que $f$ será expansível se, e somente se, $X \times X \in \mathcal{E}$.

Para começar, suponhamos que $f$ seja expansível. Nesse caso, podemos fixar $E \in \mathcal{E}$ de modo que, para todos $x, y \in X$,

$$
(x, y) \notin E \Rightarrow(f(x), f(y)) \notin\{(p, p)\} .
$$

Mas, por definição, $f(x)=p$ para todo $x \in X$. E, sendo assim, não nos resta alternativa senão tomar $E=X \times X$, do que segue imediatamente que $X \times X \in \mathcal{E}$.

Reciprocamente, se $X \times X \in \mathcal{E}$, é imediato ver que, para todos $x, y \in X$,

$$
(x, y) \notin X \times X \Rightarrow(f(x), f(y)) \notin F
$$

qualquer que seja $F \in \mathcal{E}_{p}$, a partir do que concluímos que $f$ é expansível.

c) Se $\mathcal{E}=\mathcal{P}(\Delta(X))$ e $\mathcal{F}=\mathcal{P}(\Delta(Y))$, uma função $f: X \longrightarrow Y$ será uma equivalência grosseira entre $(X, \mathcal{E})$ e $(Y, \mathcal{F})$ se, e somente se, for uma bijeção.

Demonstração. $(\Rightarrow)$ Como, por hipótese, $f$ é expansível, podemos fixar $E \in \mathcal{E}$ de tal modo que $(f(x), f(y)) \notin \Delta(Y)$ para todo $(x, y) \in(X \times X) \backslash E$. Disso resulta que, para todos $x, y \in X$,

$$
x \neq y \Rightarrow(x, y) \notin \Delta(X) \underset{E \subseteq \Delta(X)}{\Longrightarrow}(x, y) \notin E \Rightarrow(f(x), f(y)) \notin \Delta(Y) \Rightarrow f(x) \neq f(y),
$$

a partir do que concluímos que $f$ é injetora.

Por outro lado, sendo $f$ colimitada em $(Y, \mathcal{F})$, podemos fixar $F \in \mathcal{F}$ de modo que, para todo $y \in Y$, exista $x \in X$ tal que $(y, f(x)) \in F$. E, como $F \subseteq \Delta(Y)$, disso concluímos que, para cada $y \in Y$, existe $x \in X$ tal que $y=f(x)$, do que segue que $f$ é sobrejetora.

$(\Leftarrow)$ Como $f$ é injetora, $\mathcal{E}=\mathcal{P}(\Delta(X))$ e $\mathcal{F}=\mathcal{P}(\Delta(Y))$, segue dos exemplos 4.4.3-b) e 4.4.11-b) que $f$ é bornológica e expansível. E, uma vez que $f$ é, também, sobrejetora (e, portanto, colimitada), disso resulta, por 4.4.31, que $f$ é uma equivalência grosseira.

d) Se $A \subseteq X$ for colimitado em $(X, \mathcal{E})$, então $\left(A, \mathcal{E}_{A}\right)$ e $(X, \mathcal{E})$ serão grosseiramente equivalentes.

Demonstração. Com efeito, dado um conjunto colimitado $A \subseteq X$, é imediato ver que a função $i: A \longrightarrow X$ tal que $i(a)=a$ é, trivialmente, bornológica e expansível. E, uma vez que $\operatorname{Im}(i)=i(A)=A$, e, por hipótese, $A$ é colimitado em $(X, \mathcal{E})$, é igualmente fácil ver que $i$ é, também, colimitada, a partir do que concluímos, por 4.4.31, que $i$ é uma equivalência grosseira. 
e) Segue do exemplo anterior e das observações 4.1.13 e 4.4.24-b) que, para toda pseudométrica $d$ sobre $X$ e todo subconjunto $A \subseteq X$ denso em $\left(X, \tau_{d}\right)$, os espaços $\left(X, \mathcal{E}_{d}\right)$ e $\left(A, \mathcal{E}_{\left.d\right|_{A \times A}}\right)$ são grosseiramente equivalentes.

(Observe que, em particular, isso mostra que $\left(\mathbb{R}, \mathcal{E}_{d_{|\cdot|}}\right)$ e $\left(\mathbb{Q}, \mathcal{E}_{d_{|\cdot| \mathbb{Q}}}\right)$ são grosseiramente equivalentes.)

OBSERVAÇÃo 4.4.34. Dada uma função $f: X \longrightarrow Y$, não é difícil mostrar que $f$ será bornológica em relação a $\mathcal{E}$ e $\mathcal{F}$ se, e somente se, a função $f^{\prime}: X \longrightarrow \operatorname{Im}(f)$ tal que $f^{\prime}(x)=f(x)$ for bornológica em relação a $\mathcal{E}$ e $\mathcal{F}_{\operatorname{Im}(f)}$. Isso segue do fato de que, para todo $F \in \mathcal{F}$ e todo $(x, y) \in X \times X$,

$$
(f(x), f(y)) \in F \Leftrightarrow(f(x), f(y)) \in F \cap(\operatorname{Im}(f) \times \operatorname{Im}(f)),
$$

e o mesmo se aplica à expansividade.

A partir do teorema 4.4.31 e da observação 4.4.34, obtemos, facilmente, o próximo resultado, o qual é, em essência, uma caracterização dos mergulhos grosseiros.

TeOrema 4.4.35. Uma função $f: X \longrightarrow Y$ é um mergulho grosseiro se, e somente se, for bornológica e expansivel.

OBSERVAÇÃo 4.4.36. Observe que, pelos teoremas 4.4.31 e 4.4.35, toda equivalência grosseira é, em particular, um mergulho grosseiro.

Os dois próximos corolários são consequências imediatas do teorema 4.4.35, do corolário 4.4.18, e das proposições 4.4.5 e 4.4.12.

Corolário 4.4.37. A composição de mergulhos grosseiros é um mergulho grosseiro.

Corolário 4.4.38. A inversa de um mergulho grosseiro bijetor é, também, um mergulho grosseiro.

E, uma vez que toda equivalência grosseira é, em particular, um mergulho grosseiro, e, pela proposição 4.4.22, todo mergulho grosseiro sobrejetor é, na verdade, uma equivalência grosseira, vale, também, o seguinte resultado:

Corolário 4.4.39. A inversa de uma equivalência grosseira bijetora é, também, equivalência grosseira.

Por fim, vamos discutir um pouco mais a fundo a composição de equivalências grosseiras. A partir da observação 4.4.36, do corolário 4.4.37 e da proposição 4.4.22, podemos concluir que a composição de equivalências grosseiras sobrejetoras é, também, uma equivalência grosseira. A seguir, mostraremos que isso também vale em geral.

Proposição 4.4.40. A composição de equivalências grosseiras é uma equivalência grosseira.

Demonstração. Dados espaços coarse $(X, \mathcal{E}),(Y, \mathcal{F})$ e $(Z, \mathcal{G})$, e equivalências grosseiras

$$
f: X \longrightarrow Y \quad \text { e } \quad g: Y \longrightarrow Z
$$

vamos mostrar que $g \circ f: X \longrightarrow Z$ é uma equivalência grosseira.

Para isso, inicialmente observe que, de 4.4.36 e 4.4.37, segue que $g \circ f$ é um mergulho grosseiro. E, sendo assim, é suficiente mostrarmos que $g \circ f$ é colimitada em $(Z, \mathcal{G})$. 
Como, por hipótese, $g$ é colimitada em $(Z, \mathcal{G})$, podemos fixar $G \in \mathcal{G}$ de modo que, para todo $z \in Z$, exista $y \in Y$ tal que $(z, g(y)) \in G$. Analogamente, como $f$ é colimitada em $(Y, \mathcal{F})$, podemos tomar $F \in \mathcal{F}$ de modo que, para cada $y \in Y$, exista $x \in X$ tal que $(y, f(x)) \in F$. Como $g$ é bornológica, segue da proposição 4.4 .8 que $F^{g}$ pertence a $\mathcal{G}$. E, sendo $G$ um elemento de $\mathcal{G}$, disso concluímos que $F^{g} \circ G$ também pertence a $\mathcal{G}$.

Vamos mostrar, a seguir, que, para todo $z \in Z$, existe $x \in X$ tal que $(z,(g \circ f)(x)) \in F^{g} \circ G$, a partir do que seguirá que $g \circ f$ é colimitada em $(Z, \mathcal{G})$.

Com efeito, dado $z \in Z$, podemos tomar $y \in Y$ tal que $(z, g(y)) \in G$. Do mesmo modo, podemos fixar $x \in X$ tal que $(y, f(x)) \in F$. Mas

$$
(y, f(x)) \in F \Rightarrow(g(y), g(f(x))) \in F^{g} .
$$

E, por sua vez,

$$
\left[(z, g(y)) \in G \text { e }(g(y), g(f(x))) \in F^{g}\right] \Rightarrow(z, g(f(x))) \in F^{g} \circ G \Rightarrow(z,(g \circ f)(x)) \in F^{g} \circ G .
$$

Consideremos a relação $\sim$, definida sobre a classe de todos os pares de espaços coarse de modo que $(X, \mathcal{E}) \sim(Y, \mathcal{F})$ se, e somente se, $(X, \mathcal{E})$ é grosseiramente equivalente a $(Y, \mathcal{F})$. Decorre imediatamente dos itens a) e b) da observação 4.4.20 e da proposição 4.4 .40 que é, respectivamente, reflexiva, simétrica e transitiva, sendo, portanto, uma relação de equivalência. Disso resulta que espaços grosseiramente equivalentes são, em essência, representantes de uma mesma classe de equivalência de espaços coarse.

No que resta desta seção, vamos nos dedicar ao estudo de um tipo especial de função entre um espaço coarse e um espaço uniforme. Para isso, fixemos um espaço coarse $(X, \mathcal{E})$ e um espaço uniforme $(Y, \mathscr{F})$.

DefiniçÃo 4.4.41 (FunÇÃo NÃO COlAPSADA). Uma função $f: X \longrightarrow Y$ é dita não colapsada em relação a $\mathcal{E}$ e $\mathscr{F}$ se existirem $E \in \mathcal{E}$ e $F \in \mathscr{F}$ tais que, para todo $(x, y) \in X \times X$,

$$
(x, y) \notin E \Rightarrow(f(x), f(y)) \notin F .
$$

OBSERVAÇÃo 4.4.42. Note que podemos supor, sem perda de generalidade, que os subconjuntos $E$ e $F$ da definição 4.4 .41 pertencem a bases $\mathscr{B}$ e $\mathscr{C}$ de $\mathcal{E}$ e de $\mathscr{F}$, respectivamente. De fato, como $\mathscr{B}$ é base de $\mathcal{E}$, podemos fixar $B \in \mathscr{B}$ tal que $E \subseteq B$. Analogamente, como $\mathscr{C}$ é base de $\mathscr{F}$, podemos tomar $C \in \mathscr{C}$ tal que $C \subseteq F$. Dessa forma, para todo $(x, y) \in X \times X$,

$$
(x, y) \notin B \Rightarrow(x, y) \notin E \Rightarrow(f(x), f(y)) \notin F \Rightarrow(f(x), f(y)) \notin C .
$$

EXEMPLO 4.4.43. Vejamos como fica a definição 4.4.41 no caso em que $\mathcal{E}$ e $\mathscr{F}$ são induzidas por pseudométricas $d$ e $\partial$, respectivamente. Vamos mostrar que, nesse caso, uma função $f: X \longrightarrow Y$ é não colapsada se, e somente se, existem $\Delta, M>0$ tais que $\partial(f(x), f(y)) \geq M$ para quaisquer $x, y \in X$ tais que $d(x, y) \geq \Delta$.

Para isso, consideremos as bases $\mathscr{B}:=\left\{E_{\Delta}^{d}: \Delta>0\right\}$ e $\mathscr{C}:=\left\{F_{M}^{\partial}: M>0\right\}$ de $\mathcal{E}$ e de $\mathscr{F}$, respectivamente.

$(\Rightarrow)$ Como $f$ é não colapsada, segue da observação 4.4 .42 que existem $\Delta, M>0$ tais que $(f(x), f(y)) \in(Y \times Y) \backslash F_{M}^{\partial}$ para todo $(x, y) \in(X \times X) \backslash E_{\Delta}^{d}$. Dessa forma, para quaisquer $x, y \in X$,

$$
d(x, y) \geq \Delta \Rightarrow(x, y) \notin E_{\Delta}^{d} \Rightarrow(f(x), f(y)) \notin F_{M}^{\partial} \Rightarrow \partial(f(x), f(y)) \geq M .
$$


$(\Leftarrow)$ Sejam $\Delta, M>0$ tais que $\partial(f(x), f(y)) \geq M$ para todos $x, y \in X$ tais que $d(x, y) \geq \Delta$. Note que, para todo $(x, y) \in X \times X$,

$$
(x, y) \notin E_{\Delta}^{d} \Rightarrow d(x, y) \geq \Delta \Rightarrow \partial(f(x), f(y)) \geq M \Rightarrow(f(x), f(y)) \notin F_{M}^{\partial} .
$$

E, portanto, $f$ é não colapsada.

ObservaÇÃo 4.4.44. Sejam $(X, d)$ e $(Y, \partial)$ espaços pseudométricos, $f: X \longrightarrow Y$ uma função não colapsada, e $\Delta, M>0$ tais que $\partial(f(x), f(y)) \geq M$ para todos $x, y \in X$ tais que $d(x, y) \geq \Delta$. Nessas condições, dados $\Delta^{\prime}, M^{\prime}>0$ tais que $\Delta^{\prime} \geq \Delta$ e $M^{\prime} \leq M$, é imediato ver que, para todos $x, y \in X$,

$$
d(x, y) \geq \Delta^{\prime} \Rightarrow d(x, y) \geq \Delta \Rightarrow \partial(f(x), f(y)) \geq M \Rightarrow \partial(f(x), f(y)) \geq M^{\prime} .
$$

Por fim, observe que as funções expansíveis e os mergulhos uniformes entre espaços pseudométricos são, trivialmente, funções não colapsadas. 


\section{Capítulo 5}

\section{Mergulhos ENTRE ESPAÇOS DE BANACH}

Neste capítulo, voltaremos nossa atenção ao estudo dos mergulhos uniformes e mergulhos grosseiros entre espaços de Banach, e de como eles se relacionam entre si. O principal objetivo, aqui, é enunciar e demonstrar alguns resultados recentes de Christian Rosendal [Ros17b] acerca da existência de mergulhos uniformes e mergulhos grosseiros entre espaços de Banach. Tais resultados são uma contribuição à geometria não linear dos espaços de Banach, e ajudam a responder a uma questão básica levantada por Kalton sobre mergulhos. Para que possamos compreender seus enunciados, vamos, inicialmente, introduzir os espaços $\left(E \times F,\|\cdot\|_{E \times F}\right)$ e $\left(\ell_{p}\left(E,\|\cdot\|_{E}\right),\|\cdot\|_{p}\right)$, em que $\left(E,\|\cdot\|_{E}\right)$ e $\left(F,\|\cdot\|_{F}\right)$ são espaços normados sobre $\mathbb{R}$ ou $\mathbb{C}$ e $p \in$ $[1,+\infty]$. Isso será feito nas primeiras duas seções do capítulo. Uma vez munidos do ferramental matemático apropriado, poderemos, enfim, demonstrar os resultados relevantes, bem como enunciar e discutir alguns de seus corolários.

OBSERVAÇÃo 5.0.1. Ao longo do capítulo, vamos lidar, quase exclusivamente, com funções entre espaços normados. Por essa razão, sempre que nada for dito a respeito das uniformidades e estruturas coarse relevantes, assumiremos, implicitamente, que se tratam das uniformidades e estruturas coarse limitadas induzidas pelas respectivas normas sobre os espaços considerados.

\subsection{O ESPAÇO $E \times F$}

$\operatorname{Sejam~}\left(E,\|\cdot\|_{E}\right)$ e $\left(F,\|\cdot\|_{F}\right)$ espaços normados sobre um mesmo corpo $\mathbb{K}$ (em que, como sempre, $\mathbb{K}=\mathbb{R}$ ou $\mathbb{K}=\mathbb{C}$ ), e definamos sobre $E \times F$ duas operações,

$$
\begin{aligned}
+:(E \times F) \times(E \times F) & \longrightarrow E \times F \\
\left(\left(x_{1}, y_{1}\right),\left(x_{2}, y_{2}\right)\right) & \longmapsto\left(x_{1}+y_{1}, x_{2}+y_{2}\right)
\end{aligned}
$$

e

$$
\begin{aligned}
\cdot: \mathbb{K} \times(E \times F) & \longrightarrow E \times F \\
(\lambda,(x, y)) & \longmapsto(\lambda x, \lambda y),
\end{aligned}
$$

de adição e de multiplicação por escalar, respectivamente.

Observe, inicialmente, que + e $\cdot$ estão bem definidas, uma vez que as respectivas operações de adição e multiplicação por escalar sobre $E$ e $F$ estão bem definidas. Também não é difícil mostrar que $+\mathrm{e} \cdot$ satisfazem as oito propriedades definidoras de espaço vetorial, as quais seguem 
diretamente das respectivas propriedades de $E$ e de $F$ como espaços vetoriais sobre $\mathbb{K}$, e, a partir disso, concluir que essas operações equipam $E \times F$ com uma estrutura de $\mathbb{K}$ - espaço vetorial.

A seguir, mostraremos que a função $\|\cdot\|_{E \times F}: E \times F \longrightarrow \mathbb{R}$ tal que

$$
\|(x, y)\|_{E \times F}=\|x\|_{E}+\|y\|_{F}
$$

é uma norma sobre $E \times F$. Para isso, precisamos verificar que $\|\cdot\|_{E \times F}$ satisfaz as propriedades de (1) a (4) da definição 2.6.1 quaisquer que sejam $u:=(x, y)$ e $v:=\left(x^{\prime}, y^{\prime}\right)$ em $E \times F$ e $\lambda \in \mathbb{K}$.

Inicialmente, note que

$$
\|u\|_{E \times F}=\|x\|_{E}+\|y\|_{F} \geq 0
$$

pois, por hipótese, $\|x\|_{E},\|y\|_{F} \geq 0$. E, uma vez que $\|x\|_{E}=\|y\|_{F}=0$ se, e somente se, $x=0_{E}$ e $y=0_{F}$, também é fácil ver que

$$
\|u\|_{E \times F}=0 \Leftrightarrow \underbrace{\|x\|_{E}}_{\geq 0}+\underbrace{\|y\|_{F}}_{\geq 0}=0 \Leftrightarrow\|x\|_{E}=\|y\|_{F}=0 \Leftrightarrow u=\left(0_{E}, 0_{F}\right)=0_{E \times F} .
$$

Além disso, segue da definição de $\|\cdot\|_{E \times F}$ e das propriedades de $\|\cdot\|_{E}$ e de $\|\cdot\|_{F}$ que

$$
\begin{aligned}
\|\lambda \cdot u\|_{E \times F} & =\|(\lambda x, \lambda y)\|_{E \times F}=\|\lambda x\|_{E}+\|\lambda y\|_{F}=|\lambda| \cdot\|x\|_{E}+|\lambda| \cdot\|y\|_{F} \\
& =|\lambda|\left(\|x\|_{E}+\|y\|_{F}\right)=|\lambda| \cdot\|u\|_{E \times F}
\end{aligned}
$$

e que

$$
\begin{aligned}
\|u+v\|_{E \times F} & =\left\|\left(x+x^{\prime}, y+y^{\prime}\right)\right\|_{E \times F}=\left\|x+x^{\prime}\right\|_{E}+\left\|y+y^{\prime}\right\|_{F} \\
& \leq\left(\|x\|_{E}+\left\|x^{\prime}\right\|_{E}\right)+\left(\|y\|_{F}+\left\|y^{\prime}\right\|_{F}\right) \\
& =\left(\|x\|_{E}+\|y\|_{F}\right)+\left(\left\|x^{\prime}\right\|_{E}+\left\|y^{\prime}\right\|_{F}\right) \\
& =\|u\|_{E \times F}+\|v\|_{E \times F} .
\end{aligned}
$$

E, como $u, v \in E \times F$ e $\lambda \in \mathbb{K}$ são arbitrários, isso conclui a demonstração.

OBSERVAÇÃo 5.1.1. Na verdade, é possível mostrar que $\|\cdot\|_{p}: E \times F \longrightarrow \mathbb{R}$ tal que

$$
\|(x, y)\|_{p}=\left(\|x\|_{E}^{p}+\|y\|_{F}^{p}\right)^{\frac{1}{p}}
$$

é uma norma sobre $E \times F$ para todo $p \in[1, \infty[$. E não é difícil ver que essas normas são todas equivalentes à norma $\|\cdot\|_{\infty}: E \times F \longrightarrow \mathbb{R}$ tal que

$$
\|(x, y)\|_{\infty}=\max \left\{\|x\|_{E},\|y\|_{F}\right\}
$$

e, portanto, equivalentes entre si.

Vamos, agora, tecer algumas considerações sobre a completude de $\left(E \times F,\|\cdot\|_{E \times F}\right)$. Para isso, vamos nos valer de dois fatos simples a respeito de uma sequência $\left(x_{n}, y_{n}\right)_{n \in \mathbb{N}}$ de elementos de $E \times F$, cuja demonstração deixamos a cargo leitor.

FATO 1 A sequência $\left(x_{n}, y_{n}\right)_{n \in \mathbb{N}}$ é de Cauchy em $\left(E \times F,\|\cdot\|_{E \times F}\right)$ se, e somente se, $\left(x_{n}\right)_{n \in \mathbb{N}}$ e $\left(y_{n}\right)_{n \in \mathbb{N}}$ são sequências de Cauchy em $\left(E,\|\cdot\|_{E}\right)$ e em $\left(F,\|\cdot\|_{F}\right)$, respectivamente.

FATO 2 A sequência $\left(x_{n}, y_{n}\right)_{n \in \mathbb{N}}$ converge em $\left(E \times F,\|\cdot\|_{E \times F}\right)$ se, e somente se, $\left(x_{n}\right)_{n \in \mathbb{N}}$ e $\left(y_{n}\right)_{n \in \mathbb{N}}$ convergem, respectivamente, em $\left(E,\|\cdot\|_{E}\right)$ e em $\left(F,\|\cdot\|_{F}\right)$.

A partir desses dois fatos, podemos, facilmente, deduzir o seguinte resultado: 
Proposição 5.1.2. O espaço $\left(E \times F,\|\cdot\|_{E \times F}\right)$ será um espaço de Banach sempre que $\left(E,\|\cdot\|_{E}\right)$ $e\left(F,\|\cdot\|_{F}\right)$ forem espaços de Banach.

Demonstração. Sejam $\left(E,\|\cdot\|_{E}\right)$ e $\left(F,\|\cdot\|_{F}\right)$ espaços de Banach, e seja $\left(x_{n}, y_{n}\right)_{n \in \mathbb{N}}$ uma sequência de Cauchy arbitrária em $\left(E \times F,\|\cdot\|_{E \times F}\right)$. Observe que, pelo FATO 1 , disso resulta que $\left(x_{n}\right)_{n \in \mathbb{N}}$ e $\left(y_{n}\right)_{n \in \mathbb{N}}$ são sequências de Cauchy em $\left(E,\|\cdot\|_{E}\right)$ e $\left(F,\|\cdot\|_{F}\right)$, respectivamente. E como, por hipótese, $\left(E,\|\cdot\|_{E}\right)$ e $\left(F,\|\cdot\|_{F}\right)$ são espaços de Banach, disso concluímos que $\left(x_{n}\right)_{n \in \mathbb{N}}$ e $\left(y_{n}\right)_{n \in \mathbb{N}}$ são ambas convergentes. Assim sendo, segue do FATO 2 que $\left(x_{n}, y_{n}\right)_{n \in \mathbb{N}}$ converge em $\left(E \times F,\|\cdot\|_{E \times F}\right)$. E, uma vez que $\left(x_{n}, y_{n}\right)_{n \in \mathbb{N}}$ é arbitrária, isso prova que $\left(E \times F,\|\cdot\|_{E \times F}\right)$ é, ele próprio, um espaço de Banach.

Seja $\left(X,\|\cdot\|_{X}\right)$ um espaço normado. Dadas funções $f: X \longrightarrow E$ e $g: X \longrightarrow F$, podemos definir a função $f \oplus g: X \longrightarrow E \times F$ tal que $(f \oplus g)(x)=(f(x), g(x))$. Com isso em mente, alguém poderia perguntar o que podemos inferir a respeito de $f \oplus g$ conhecidas as propriedades de $f$ e de $g$. Ou, de forma mais precisa:

Quais propriedades de $f$ e de $g$ são "herdadas" por $f \oplus g$ ?

Na proposição a seguir, veremos que a continuidade uniforme é uma delas (veja o teorema 4.4.6).

Proposição 5.1.3. Sejam $\left(X,\|\cdot\|_{X}\right),\left(E,\|\cdot\|_{E}\right)$ e $\left(F,\|\cdot\|_{F}\right)$ espaços normados, e $f: X \longrightarrow E$, $g: X \longrightarrow F$ funções uniformemente continuas. Nessas condições, a função $f \oplus g: X \longrightarrow E \times F$ tal que $(f \oplus g)(x)=(f(x), g(x))$ é, também, uniformemente continua.

Demonstração. Seja $\varepsilon>0$ dado. Como $f$ e $g$ são uniformemente contínuas, existem $\delta_{1}, \delta_{2}>0$ tais que, para todos $x, y \in X$,

$$
\|x-y\|_{X}<\delta_{1} \Rightarrow\|f(x)-f(y)\|_{E}<\frac{\varepsilon}{2},
$$

e

$$
\|x-y\|_{X}<\delta_{2} \Rightarrow\|g(x)-g(y)\|_{F}<\frac{\varepsilon}{2} .
$$

Assim sendo, se tomarmos $\delta:=\min \left\{\delta_{1}, \delta_{2}\right\}$, teremos que

$$
\begin{aligned}
\|(f \oplus g)(x)-(f \oplus g)(y)\|_{E \times F} & =\|(f(x)-f(y), g(x)-g(y))\|_{E \times F} \\
& =\|f(x)-f(y)\|_{E}+\|g(x)-g(y)\|_{F} \\
& <\frac{\varepsilon}{2}+\frac{\varepsilon}{2}=\varepsilon
\end{aligned}
$$

para todos $x, y \in X$ tais que $\|x-y\|_{X}<\delta$. E, como $\varepsilon>0$ é arbitrário, disso resulta que $f \oplus g$ é uniformemente contínua.

De modo análogo, mostra-se que, se $f$ e $g$ forem mergulhos uniformes, então $f \oplus g$ será um mergulho uniforme, o mesmo valendo para a expansividade.

\section{$5.2 \mathrm{O} \operatorname{ESPAÇO} \ell_{p}(E)$}

A seguir, vamos introduzir o espaço $\ell_{p}(E,\|\cdot\|)$, em que $p \in[1,+\infty]$ e $(E,\|\cdot\|)$ é um espaço normado sobre $\mathbb{K}$. Ao longo da seção, assumiremos conhecidos alguns resultados básicos a respeito dos espaços $\ell_{p}$, em que $p \in[1,+\infty]$, os quais são usualmente estudados em um primeiro curso de Análise Funcional. 
DeFINIÇÃo 5.2.1. Dados $p \in[1,+\infty]$ e um espaço normado $(E,\|\cdot\|)$ sobre $\mathbb{K}$, denotaremos por $\ell_{p}(E,\|\cdot\|)$ o conjunto

$$
\left\{\left(x_{n}\right)_{n \in \mathbb{N}}: x_{n} \in E \forall n \in \mathbb{N} \text { e }\left(\left\|x_{n}\right\|\right)_{n \in \mathbb{N}} \in \ell_{p}\right\} .
$$

Quando a norma $\|\cdot\|$ sobre $E$ estiver clara pelo contexto, escreveremos apenas $\ell_{p}(E)$ para nos referirmos a $\ell_{p}(E,\|\cdot\|)$.

Sejam $p \in[1,+\infty]$ e $(E,\|\cdot\|)$ um espaço normado sobre $\mathbb{K}$. A partir da definição 5.2.1, fica claro que as propriedades de $\ell_{p}(E)$ devem depender, essencialmente, das propriedades de $(E,\|\cdot\|)$ e de $\ell_{p}$. Observe ainda que, se $E=\mathbb{K}$, teremos, simplesmente, $\ell_{p}(E)=\ell_{p}$.

No que segue, vamos examinar, em detalhes, o caso em que $p \in[1,+\infty[$. Como primeiro passo, mostraremos que $\ell_{p}(E)$ é um espaço vetorial em relação à soma e multiplicação por escalar usuais de funções. Inicialmente, precisamos verificar que as operações

$$
+: \ell_{p}(E) \times \ell_{p}(E) \longrightarrow \ell_{p}(E) \quad \text { e } \quad \cdot: \mathbb{K} \times \ell_{p}(E) \longrightarrow \ell_{p}(E)
$$

tais que

$$
\left(x_{n}\right)_{n \in \mathbb{N}}+\left(y_{n}\right)_{n \in \mathbb{N}}=\left(x_{n}+y_{n}\right)_{n \in \mathbb{N}}
$$

$\mathrm{e}$

$$
\lambda \cdot\left(x_{n}\right)_{n \in \mathbb{N}}=\left(\lambda x_{n}\right)_{n \in \mathbb{N}}
$$

estão bem definidas. Para isso, é suficiente mostrarmos que $\left(x_{n}+\lambda y_{n}\right)_{n \in \mathbb{N}} \in \ell_{p}(E)$ quaisquer que sejam $\left(x_{n}\right)_{n \in \mathbb{N}},\left(y_{n}\right)_{n \in \mathbb{N}} \in \ell_{p}(E)$ e $\lambda \in \mathbb{K}$.

Comecemos tomando $\left(x_{n}\right)_{n \in \mathbb{N}},\left(y_{n}\right)_{n \in \mathbb{N}} \in \ell_{p}(E)$ e $\lambda \in \mathbb{K}$ de modo arbitrário. Como $E$ é um espaço vetorial, temos que $x_{n}+\lambda y_{n} \in E$ para todo $n \in \mathbb{N}$. Além disso, segue da desigualdade triangular que

$$
\left\|x_{n}+\lambda y_{n}\right\|^{p} \leq\left(\left\|x_{n}\right\|+|\lambda|\left\|y_{n}\right\|\right)^{p} \quad \forall n \in \mathbb{N} .
$$

Por outro lado,

$$
\left(x_{n}\right)_{n \in \mathbb{N}},\left(y_{n}\right)_{n \in \mathbb{N}} \in \ell_{p}(E) \Rightarrow\left(\left\|x_{n}\right\|\right)_{n \in \mathbb{N}},\left(\left\|y_{n}\right\|\right)_{n \in \mathbb{N}} \in \ell_{p}
$$

Como $\ell_{p}$ é um espaço vetorial, disso resulta que $\left(\left\|x_{n}\right\|+|\lambda|\left\|y_{n}\right\|\right)_{n \in \mathbb{N}} \in \ell_{p}$, a partir do que concluímos que $\sum_{n=1}^{+\infty}\left(\left\|x_{n}\right\|+|\lambda|\left\|y_{n}\right\|\right)^{p}$ converge. Logo, segue do Critério da comparação (proposição 2.4.3) que $\sum_{n=1}^{+\infty}\left\|x_{n}+\lambda y_{n}\right\|^{p}$ converge e que

$$
\sum_{n=1}^{+\infty}\left\|x_{n}+\lambda y_{n}\right\|^{p} \leq \sum_{n=1}^{+\infty}\left(\left\|x_{n}\right\|+|\lambda|\left\|y_{n}\right\|\right)^{p}
$$

Isso mostra que $\left(x_{n}+\lambda y_{n}\right)_{n \in \mathbb{N}} \in \ell_{p}(E)$, concluindo a demonstração.

A seguir, devemos verificar que $+\mathrm{e} \cdot$ satisfazem as oito propriedades definidoras de espaço vetorial. No entanto, como a adição e a multiplicação por escalar em $\ell_{p}(E)$ são realizadas "componente a componente", é imediato ver que estas seguem diretamente das respectivas propriedades de $E$ como espaço vetorial sobre $\mathbb{K}$. 
Nosso próximo passo é mostrar que a função $\|\cdot\|_{p}: \ell_{p}(E) \longrightarrow \mathbb{R}$ definida de modo que

$$
\left\|\left(x_{n}\right)_{n \in \mathbb{N}}\right\|_{p}=\left(\sum_{n=1}^{+\infty}\left\|x_{n}\right\|^{p}\right)^{\frac{1}{p}}
$$

é uma norma sobre $\ell_{p}(E)$. Para isso, devemos verificar que $\|\cdot\|_{p}$ satisfaz as condições de (1) a (4) da definição 2.6.1.

Sejam $x:=\left(x_{n}\right)_{n \in \mathbb{N}}$ e $y:=\left(y_{n}\right)_{n \in \mathbb{N}}$ em $\ell_{p}(E)$ e $\lambda \in \mathbb{K}$ dados, e denotemos por $\left(s_{n}\right)_{n \in \mathbb{N}}$ a sequência das somas parciais de $\left(\left\|x_{n}\right\|^{p}\right)_{n \in \mathbb{N}^{N}}$. Observe que $\left(s_{n}\right)_{n \in \mathbb{N}}$ é crescente e limitada. Logo, segue da proposição 2.4.1 que

$$
\|x\|_{p}^{p}:=\sum_{n=1}^{+\infty}\left\|x_{n}\right\|^{p}=\lim _{n \rightarrow+\infty} s_{n}=\sup \left\{s_{n}: n \in \mathbb{N}\right\} .
$$

Dessa forma,

$$
\left\|x_{n}\right\| \geq 0 \quad \forall n \in \mathbb{N} \Rightarrow s_{n} \geq 0 \quad \forall n \in \mathbb{N} \Rightarrow\|x\|_{p}^{p}=\sup \left\{s_{n}: n \in \mathbb{N}\right\} \geq 0 \Rightarrow\|x\|_{p} \geq 0 .
$$

Além disso,

$$
\begin{gathered}
\|x\|_{p}=0 \Rightarrow \sum_{n=1}^{+\infty}\left\|x_{n}\right\|^{p}=0 \\
\Rightarrow 0 \leq x_{n} \leq \sup \left\{x_{n}: n \in \mathbb{N}\right\}=0 \quad \forall n \in \mathbb{N} \\
\Rightarrow x_{n}=0 \quad \forall n \in \mathbb{N} \Rightarrow x=0 .
\end{gathered}
$$

Também é fácil ver que

$$
\begin{aligned}
\|\lambda x\|_{p} & :=\left(\sum_{n=1}^{+\infty}\left\|\lambda x_{n}\right\|^{p}\right)^{\frac{1}{p}}=\left(\sum_{n=1}^{+\infty}|\lambda|^{p}\left\|x_{n}\right\|^{p}\right)^{\frac{1}{p}}=\left(\lim _{m \rightarrow+\infty} \sum_{n=1}^{m}|\lambda|^{p}\left\|x_{n}\right\|^{p}\right)^{\frac{1}{p}} \\
& =\left(|\lambda|^{p} \lim _{m \rightarrow+\infty} \sum_{n=1}^{m}\left\|x_{n}\right\|^{p}\right)^{\frac{1}{p}}=\lambda\left(\sum_{n=1}^{+\infty}\left\|x_{n}\right\|^{p}\right)^{\frac{1}{p}}=\lambda\|x\|_{p} .
\end{aligned}
$$

Por fim, vamos mostrar que o par $(x, y)$ satisfaz a desigualdade triangular. Utilizando a equação $(*)$ para $x+1 \cdot y$, podemos concluir que

$$
\sum_{n=1}^{+\infty}\left\|x_{n}+y_{n}\right\|^{p} \leq \sum_{n=1}^{+\infty}\left(\left\|x_{n}\right\|+\left\|y_{n}\right\|\right)^{p}
$$

Mas, pela desigualdade triangular em $\ell_{p}$ (em relação à norma usual), temos que

$$
\left(\sum_{n=1}^{+\infty}\left(\left\|x_{n}\right\|+\left\|y_{n}\right\|\right)^{p}\right)^{\frac{1}{p}} \leq\left(\sum_{n=1}^{+\infty}\left\|x_{n}\right\|^{p}\right)^{\frac{1}{p}}+\left(\sum_{n=1}^{+\infty}\left\|y_{n}\right\|^{p}\right)^{\frac{1}{p}}
$$


Logo:

$$
\begin{aligned}
\|x+y\|_{p} & :=\left(\sum_{n=1}^{+\infty}\left\|x_{n}+y_{n}\right\|^{p}\right)^{\frac{1}{p}} \leq\left(\sum_{n=1}^{+\infty}\left(\left\|x_{n}\right\|+\left\|y_{n}\right\|\right)^{p}\right)^{\frac{1}{p}} \\
& \leq\left(\sum_{n=1}^{+\infty}\left\|x_{n}\right\|^{p}\right)^{\frac{1}{p}}+\left(\sum_{n=1}^{+\infty}\left\|y_{n}\right\|^{p}\right)^{\frac{1}{p}}=\|x\|_{p}+\|y\|_{p} .
\end{aligned}
$$

Como $x, y \in \ell_{p}(E)$ e $\lambda \in \mathbb{K}$ são arbitrários, isso completa a prova de que $\|\cdot\|_{p}$ é uma norma sobre $\ell_{p}(E)$.

Observe o papel crucial desempenhado por $\|\cdot\|$ na definição de $\ell_{p}(E,\|\cdot\|)$. Tendo isso em mente, e após alguma reflexão, a seguinte questão surge naturalmente:

Se $\|\cdot\|_{1}$ e $\|\cdot\|_{2}$ forem normas equivalentes sobre $E$, o que podemos dizer a respeito de $\ell_{p}\left(E,\|\cdot\|_{1}\right)$ e $\ell_{p}\left(E,\|\cdot\|_{2}\right)$ ?

E, mais ainda:

Como as normas $\|\cdot\|_{p}^{1}$ e $\|\cdot\|_{p}^{2}$, induzidas por $\|\cdot\|_{1}$ e $\|\cdot\|_{2}$ sobre $\ell_{p}\left(E,\|\cdot\|_{1}\right)$ e $\ell_{p}\left(E,\|\cdot\|_{2}\right)$, respectivamente, estão relacionadas?

A proposição 5.2.2 nos dá a resposta a essas indagações.

Proposição 5.2.2. Sejam E um $\mathbb{K}$ - espaço vetorial, $\|\cdot\|_{1},\|\cdot\|_{2}: E \longrightarrow \mathbb{R}$ normas equivalentes sobre $E, p \in\left[1,+\infty\left[\right.\right.$ dado e $\|\cdot\|_{p}^{1}$ e $\|\cdot\|_{p}^{2}$ as normas induzidas por $\|\cdot\|_{1}$ e $\|\cdot\|_{2}$ sobre $\ell_{p}\left(E,\|\cdot\|_{1}\right)$ e $\ell_{p}\left(E,\|\cdot\|_{2}\right)$, respectivamente. Nessas condições:

a) $\ell_{p}\left(E,\|\cdot\|_{1}\right)=\ell_{p}\left(E,\|\cdot\|_{2}\right)$

b) as normas $\|\cdot\|_{p}^{1}$ e $\|\cdot\|_{p}^{2}$ são equivalentes.

Demonstração. a) Como $\|\cdot\|_{1}$ e $\|\cdot\|_{2}$ são equivalentes, ${ }^{1}$ existem $A, B>0$ tais que

$$
A\|x\|_{1} \leq\|x\|_{2} \leq B\|x\|_{1} \quad \forall x \in E .
$$

Seja $x \in \ell_{p}\left(E,\|\cdot\|_{1}\right)$ dado. Como a função $f:\left[0,+\infty\left[\longrightarrow \mathbb{R}\right.\right.$ tal que $f(u)=u^{p}$ é estritamente crescente, segue de $(*)$ que

$$
A^{p}\left\|x_{n}\right\|_{1}^{p} \leq\left\|x_{n}\right\|_{2}^{p} \leq B^{p}\left\|x_{n}\right\|_{1}^{p} \quad \forall n \in \mathbb{N} .
$$

Por sua vez, como $\sum_{n=1}^{+\infty}\left\|x_{n}\right\|_{1}^{p}$ converge $\left(\right.$ pois $\left.x \in \ell_{p}\left(E,\|\cdot\|_{1}\right)\right)$, segue do Critério da comparação que $\sum_{n=1}^{+\infty}\left\|x_{n}\right\|_{2}^{p}$ converge e que

$$
\sum_{n=1}^{+\infty}\left\|x_{n}\right\|_{2}^{p} \leq B^{p} \sum_{n=1}^{+\infty}\left\|x_{n}\right\|_{1}^{p}
$$

Disso resulta, em particular, que $x \in \ell_{p}\left(E,\|\cdot\|_{2}\right)$. E, como $x \in \ell_{p}\left(E,\|\cdot\|_{1}\right)$ é arbitrário, isso mostra que $\ell_{p}\left(E,\|\cdot\|_{1}\right) \subseteq \ell_{p}\left(E,\|\cdot\|_{2}\right)$.

\footnotetext{
${ }^{1}$ Veja a definição 2.7.30.
} 
Por outro lado, observe que, para todo $x \in E$,

$$
A\|x\|_{1} \leq\|x\|_{2} \leq B\|x\|_{1} \Leftrightarrow \frac{1}{B}\|x\|_{2} \leq\|x\|_{1} \leq \frac{1}{A}\|x\|_{2} .
$$

Dessa forma, basta repetirmos o argumento acima substituindo 1 por 2,2 por 1 e $B$ por $\frac{1}{A}$ para concluírmos que $\ell_{p}\left(E,\|\cdot\|_{2}\right)=\ell_{p}\left(E,\|\cdot\|_{1}\right)$ e que

$$
\sum_{n=1}^{+\infty}\left\|x_{n}\right\|_{1}^{p} \leq \frac{1}{A^{p}} \sum_{n=1}^{+\infty}\left\|x_{n}\right\|_{2}^{p}
$$

para toda sequência $\left(x_{n}\right)_{n \in \mathbb{N}} \in \ell_{p}\left(E,\|\cdot\|_{1}\right)=\ell_{p}\left(E,\|\cdot\|_{2}\right) .^{2}$

b) Seja $Y=\ell_{p}\left(E,\|\cdot\|_{1}\right)=\ell_{p}\left(E,\|\cdot\|_{2}\right)$. Segue das equações (1) e (2) (deduzidas acima), que

$$
A^{p} \sum_{n=1}^{+\infty}\left\|x_{n}\right\|_{1}^{p} \leq \sum_{n=1}^{+\infty}\left\|x_{n}\right\|_{2}^{p} \leq B^{p} \sum_{n=1}^{+\infty}\left\|x_{n}\right\|_{1}^{p}
$$

para toda sequência $\left(x_{n}\right)_{n \in \mathbb{N}} \in Y$. Como a função $g:\left[0,+\infty\left[\longrightarrow \mathbb{R}\right.\right.$ tal que $g(u)=u^{\frac{1}{p}}$ é estritamente crescente, disso resulta que

$$
A\|x\|_{p}^{1} \leq\|x\|_{p}^{2} \leq B\|x\|_{p}^{1} \quad \forall x \in Y
$$

a partir do que concluímos que as normas $\|\cdot\|_{p}^{1}$ e $\|\cdot\|_{p}^{2}$ são equivalentes sobre $Y$.

Sejam $(E,\|\cdot\|)$ um espaço normado sobre $\mathbb{K}$ e $p \in[1,+\infty[$ um real dado. Se compararmos os espaços $\left(\ell_{p}(E),\|\cdot\|_{p}\right)$ e $\left(\ell_{p},|\cdot|_{p}\right),{ }^{3}$ veremos que, formalmente, os dois são muito parecidos, com o espaço $(E,\|\cdot\|)$ desempenhando, em $\left(\ell_{p}(E),|\cdot|_{p}\right)$ o mesmo papel de $(\mathbb{K},|\cdot|)$ em $\left(\ell_{p},|\cdot|_{p}\right)$. Como consequência disso, há uma grande semelhança operacional entre ambos. Levando isso em conta, e sabendo que $\left(\ell_{p},|\cdot|_{p}\right)$ é um espaço de Banach, o que podemos afirmar a respeito da completude de $\left(\ell_{p}(E),\|\cdot\|_{p}\right)$ ?

Aqueles que estão familiarizados com Análise Funcional provavelmente se recordarão de que um ponto crucial na demonstração de que $\left(\ell_{p},|\cdot|_{p}\right)$ é um espaço de Banach reside no fato de que o corpo $\mathbb{K}$ é completo. Tendo isso em mente, é razoável supor que a completude de $(E,\|\cdot\|)$ seja uma condição suficiente para a completude de $\left(\ell_{p}(E),\|\cdot\|_{p}\right)$. A ideia, aqui, é reproduzir a demonstração de que $\left(\ell_{p},|\cdot|_{p}\right)$ é um espaço de Banach, tomando o cuidado de substituir $\mathbb{K}$ por $E, \ell_{p}$ por $\ell_{p}(E)$ e $|\cdot|$ por $\|\cdot\|$, e de fazer as adaptações necessárias. E isso de fato funciona, como nos mostra a proposição a seguir.

Proposição 5.2.3. Sejam (E, $\|\cdot\|)$ um espaço normado sobre $\mathbb{K}$ e $p \in[1,+\infty[$ dado. Nessas condições, se $(E,\|\cdot\|)$ for um espaço de Banach, então $\left(\ell_{p}(E),\|\cdot\|_{p}\right)$ será, também, um espaço de Banach.

Demonstração. Seja $\left(x_{n}\right)_{n \in \mathbb{N}}$ uma sequência de Cauchy em $\ell_{p}(E)$ e, para cada $n \in \mathbb{N}$, sejam $x_{1}^{(n)}, x_{2}^{(n)}, \ldots \in E$ tais que $x_{n}=\left(x_{j}^{(n)}\right)_{j \in \mathbb{N}}$. Inicialmente, vamos mostrar que, para cada $j \in \mathbb{N}$, a sequência $\left(x_{j}^{(n)}\right)_{n \in \mathbb{N}}$ converge em $(E,\|\cdot\|)$. Para isso, tomemos $j \in \mathbb{N}$ e $\varepsilon>0$ de modo arbitrário.

\footnotetext{
${ }^{2}$ Embora as equações (1) e (2) não sejam utilizadas nesta demonstração em si, elas serão particularmente úteis para a demonstração do item b).

${ }^{3} \mathrm{Em}$ que $|\cdot|_{p}$ denota a norma usual de $\ell_{p}$.
} 
Como $\left(x_{n}\right)_{n \in \mathbb{N}}$ é de Cauchy, existe $N_{0} \in \mathbb{N}$ tal que $\left\|x_{m}-x_{n}\right\|_{p}<\varepsilon$ para quaisquer $m, n \in \mathbb{N}$ tais que $m, n>N_{0}$. E, como

$$
\left\|x_{j}^{(n)}-x_{j}^{(m)}\right\| \leq\left(\sum_{i=1}^{j}\left\|x_{i}^{(n)}-x_{i}^{(m)}\right\|^{p}\right)^{\frac{1}{p}} \leq\left(\sum_{i=1}^{+\infty}\left\|x_{i}^{(n)}-x_{i}^{(m)}\right\|^{p}\right)^{\frac{1}{p}}=\left\|x_{m}-x_{n}\right\|_{p} \quad \forall m, n \in \mathbb{N},
$$

disso resulta que, para todos $m, n \in \mathbb{N}$,

$$
m, n>N_{0} \Rightarrow\left\|x_{j}^{(n)}-x_{j}^{(m)}\right\|<\varepsilon .
$$

Por fim, como $\varepsilon>0$ é arbitrário, isso mostra que $\left(x_{j}^{(n)}\right)_{n \in \mathbb{N}}$ é de Cauchy e, portanto, convergente (pois $(E,\|\cdot\|)$ é um espaço de Banach).

Seja $x:=\left(x_{j}\right)_{j \in \mathbb{N}}$, em que, para cada $j \in \mathbb{N}, x_{j}:=\lim _{n \rightarrow+\infty} x_{j}^{(n)}$. Vamos mostrar que $x \in \ell_{p}(E)$ e que $x_{n} \longrightarrow x$.

Seja $\varepsilon>0$ dado. Como $\left(x_{n}\right)_{n \in \mathbb{N}}$ é de Cauchy, existe $N \in \mathbb{N}$ tal que $\left\|x_{m}-x_{n}\right\|_{p}<\frac{\varepsilon}{2^{\frac{1}{p}}}$ para quaisquer $m, n \in \mathbb{N}$ tais que $m, n>N$. Dessa forma, se $n, k \in \mathbb{N}$ são tais que $n>N$, então

$$
\sum_{j=1}^{k}\left\|x_{j}^{(n)}-x_{j}^{(m)}\right\|^{p} \leq \sum_{j=1}^{+\infty}\left\|x_{j}^{(n)}-x_{j}^{(m)}\right\|^{p}<\frac{\varepsilon^{p}}{2}
$$

para todo $m \in \mathbb{N}$ tal que $m>N$.

Pela continuidade de $\|\cdot\|$, disso concluímos que

$$
\begin{aligned}
\sum_{j=1}^{k}\left\|x_{j}^{(n)}-x_{j}\right\|^{p} & =\sum_{j=1}^{k}\left\|x_{j}^{(n)}-\lim _{m \rightarrow+\infty} x_{j}^{(m)}\right\|^{p} \\
& =\lim _{m \rightarrow+\infty}\left[\sum_{j=1}^{k}\left\|x_{j}^{(n)}-x_{j}^{(m)}\right\|^{p}\right] \leq \frac{\varepsilon^{p}}{2} .
\end{aligned}
$$

Por sua vez, como a equação $(*)$ vale para todo $k \in \mathbb{N}$, segue da proposição 2.4 .2 que $\sum_{j=1}^{+\infty}\left\|x_{j}^{(n)}-x_{j}\right\|^{p}$ converge e que

$$
\sum_{j=1}^{+\infty}\left\|x_{j}^{(n)}-x_{j}\right\|^{p} \leq \frac{\varepsilon^{p}}{2} \leq \varepsilon^{p}
$$

Em particular, disso resulta que $\left(x_{n}-x\right) \in \ell_{p}(E)$, a partir do que concluímos que $x=$ $x-\left(x_{n}-x\right) \in \ell_{p}(E)$. Além disso, como

$$
\sum_{j=1}^{+\infty}\left\|x_{j}^{(n)}-x_{j}\right\|^{p}=\left\|x_{m}-x\right\|_{p}^{p}
$$

e $n \in \mathbb{N}$ tal que $n>N$ é arbitrário, isso mostra que, para todo $n \in \mathbb{N}$,

$$
n>N \Rightarrow\left\|x_{m}-x\right\|_{p}<\varepsilon,
$$


completando a prova de que $x_{n} \longrightarrow x$.

OBservaÇÃo 5.2.4. Seja $(E,\|\cdot\|)$ um espaço normado. Explorando as propriedades de $(E,\|\cdot\|)$ e de $\ell_{\infty}$, é fácil mostrar que $\ell_{\infty}(E)$ é um espaço vetorial sobre $\mathbb{K}$ em relação à soma e à multiplicação por escalar usuais de funções e que a função $\|\cdot\|_{\infty}: \ell_{\infty}(E) \longrightarrow \mathbb{R}$ tal que

$$
\left\|\left(x_{n}\right)_{n \in \mathbb{N}}\right\|_{\infty}=\sup \left\{\left\|x_{n}\right\|: n \in \mathbb{N}\right\}
$$

é uma norma sobre $\ell_{\infty}(E)$. Também não é difícil estender as proposições 5.2.2 e 5.2.3 para o caso em que $p=\infty$. No entanto, como o espaço $\ell_{\infty}(E)$ não será essencial para o restante deste trabalho, vamos omitir essas demonstrações.

\subsection{Mergulhos ENTRE ESPAÇOS DE BANACH}

Em um artigo de 2008 [Kal08], N. J. Kalton observa que dois espaços de Banach, $\left(X,\|\cdot\|_{X}\right)$ e $\left(E,\|\cdot\|_{E}\right)$, de dimensão infinita sobre um mesmo corpo $\mathbb{K}$, e uniformemente homeomorfos, são, também, grosseiramente equivalentes. Tendo isso em vista, é natural perguntar se vale a recíproca desse resultado e se um resultado como esse se verifica também para mergulhos. Isto é:

1. Será que $\left(X,\|\cdot\|_{X}\right)$ e $\left(E,\|\cdot\|_{E}\right)$ serão uniformemente homeomorfos se, e somente se, forem grosseiramente equivalentes?

2. Se existir um mergulho uniforme de $\left(X,\|\cdot\|_{X}\right)$ em $\left(E,\|\cdot\|_{E}\right)$, podemos garantir que existe também um mergulho grosseiro?

A resposta à primeira questão é negativa. Em verdade, o próprio Kalton foi capaz de dar um exemplo de dois espaços de Banach separáveis os quais são grosseiramente equivalentes, mas não são uniformemente homeomorfos (para mais detalhes, veja [Kal12]). Contudo, a segunda questão permanece em aberto.

Nesta seção, apresentaremos alguns resultados relacionados à existência de mergulhos uniformes e mergulhos grosseiros entre espaços de Banach, a partir dos quais poderemos entender, com maior clareza, a relação entre esses dois conceitos, bem como responder à segunda questão em um caso particular. Esses resultados foram originalmente demonstrados por Christian Rosendal no artigo Equivariant geometry of Banach spaces and topological groups [Ros17b], de 2017, no qual este trabalho foi, em grande parte, inspirado. Por essa razão, as demonstrações aqui desenvolvidas seguirão bem de perto as apresentadas por Christian, com algumas poucas modificações.

Antes de mais nada, vamos provar um pequeno lema relativo à continuidade uniforme, o qual será útil mais adiante.

LEMA 5.3.1. Sejam $\left(X,\|\cdot\|_{X}\right)$ e $\left(E,\|\cdot\|_{E}\right)$ espaços normados sobre um corpo $\mathbb{K}$, e $f: X \longrightarrow E$ uma função uniformemente continua. Nessas condições. a função $g: X \longrightarrow E$ tal que $g(x)=$ $\beta \cdot f(\alpha \cdot x)$ será uniformemente contínua quaisquer que sejam $\alpha$ e $\beta$ em $\mathbb{K}$.

Demonstração. Fixemos $\alpha, \beta \in \mathbb{K}$ quaisquer, e vamos mostrar que a função $g$, definida tal como no enunciado, é uniformemente contínua. Se $\alpha=0$ ou $\beta=0$, o resultado segue trivialmente. Podemos supor, então, que $\alpha$ e $\beta$ sejam ambos não nulos. 
Seja $\varepsilon>0$ dado. Como $f$ é uniformemente contínua, existe $\delta_{1}>0$ tal que

$$
\|f(x)-f(y)\|_{E}<\frac{\varepsilon}{|\beta|}
$$

para todos $x, y \in X$ tais que $\|x-y\|_{X}<\delta_{1}$. Dessa forma, tomando $\delta=\frac{\delta_{1}}{|\alpha|}$, teremos que, quaisquer que sejam $x, y \in X$,

$$
\begin{aligned}
\|x-y\|_{X}<\delta & \Rightarrow\|\alpha \cdot x-\alpha \cdot y\|_{X}<\delta_{1} \Rightarrow\|f(\alpha \cdot x)-f(\alpha \cdot y)\|_{E}<\frac{\varepsilon}{|\beta|} \\
& \Rightarrow\|\beta \cdot f(\alpha \cdot x)-\beta \cdot f(\alpha \cdot y)\|_{E}<\varepsilon \Rightarrow\|g(x)-g(y)\|_{E}<\varepsilon
\end{aligned}
$$

E, uma vez que $\varepsilon>0$ é arbitrário, isso mostra que $g$ é uniformemente contínua.

A seguir, enunciaremos o nosso primeiro grande resultado, a partir do qual vários outros seguirão.

TeOrema 5.3.2. Sejam $\left(X,\|\cdot\|_{X}\right)$ e $\left(E,\|\cdot\|_{E}\right)$ espaços de Banach, e, para cada $n \in \mathbb{N}$, sejam $P_{n}: E \longrightarrow E$ uma projeção limitada e não nula sobre um subespaço $E_{n} \subseteq E$ e $\sigma_{n}: X \longrightarrow E_{n}$ uma função não colapsada e uniformemente continua. Suponhamos ainda que $E_{m} \subseteq \operatorname{Ker}\left(P_{n}\right)$ para quaisquer $m, n \in \mathbb{N}$ tais que $m \neq n$. Nessas condições, existe $\omega: X \longrightarrow E$ tal que $\omega$ é um mergulho uniforme e também um mergulho grosseiro.

Demonstração. Observe que podemos supor, sem perda de generalidade, que $\sigma_{n}\left(0_{X}\right)=0_{E}$ para todo $n \in \mathbb{N}$. Com efeito, dado $n \in \mathbb{N}$, é imediato ver que $\sigma_{n}^{\prime}: X \longrightarrow E_{n}$ tal que $\sigma_{n}^{\prime}(x)=$ $\sigma_{n}(x)-\sigma_{n}\left(0_{X}\right)$ está bem definida (pois $E_{n}$ é um subespaço vetorial), é uniformemente contínua e não colapsada, e é tal que $\sigma_{n}^{\prime}\left(0_{X}\right)=0_{E}$. Dessa forma, se $n \in \mathbb{N}$ for tal que $\sigma_{n}\left(0_{X}\right) \neq 0_{E}$, basta substituírmos $\sigma_{n}$ por $\sigma_{n}^{\prime}$.

Seja $n \in \mathbb{N}$ dado. Como $\sigma_{n}$ é não colapsada e uniformemente contínua, existem $\Delta_{n}, M_{n}, \delta_{n}>$ 0 tais que, para todos $x, y \in X$,

$$
\|x-y\|_{X} \geq \Delta_{n} \Rightarrow\left\|\sigma_{n}(x)-\sigma_{n}(y)\right\|_{E} \geq M_{n},
$$

e

$$
\|x-y\|_{X}<\delta_{n} \Rightarrow\left\|\sigma_{n}(x)-\sigma_{n}(y)\right\|_{E}<2^{-n} .
$$

Antes de prosseguirmos, vamos mostrar que, se $x, y \in X$ e $k \in \mathbb{N}$ são tais que $\|x-y\|_{X}<$ $k \cdot \delta_{n}$, então $\left\|\sigma_{n}(x)-\sigma_{n}(y)\right\|_{E}<k \cdot 2^{-n}$. Isso será bastante útil para o que vem a seguir.

Para cada $i \in\{0, \ldots, k\}$, seja $z_{i}=y+\frac{i}{k}(x-y)$. Observe que, para todo $i \in\{0, \ldots, k-1\}$,

$$
\left\|z_{i+1}-z_{i}\right\|_{X}=\frac{1}{k}\|x-y\|_{X}<\delta_{n}
$$

Logo, segue de (5.1) que, para cada $i \in\{0, \ldots, k-1\},\left\|\sigma_{n}\left(z_{i+1}\right)-\sigma_{n}\left(z_{i}\right)\right\|_{E}<2^{-n}$. E disso resulta que

$$
\begin{aligned}
\left\|\sigma_{n}(x)-\sigma_{n}(y)\right\|_{E} & =\left\|\sum_{i=0}^{k-1}\left(\sigma_{n}\left(z_{i+1}\right)-\sigma_{n}\left(z_{i}\right)\right)\right\|_{E} \leq \sum_{i=0}^{k-1} \underbrace{\left\|\sigma_{n}\left(z_{i+1}\right)-\sigma_{n}\left(z_{i}\right)\right\|_{E}}_{<2^{-n}} \\
& <\sum_{i=0}^{k-1} 2^{-n}=k \cdot 2^{-n},
\end{aligned}
$$


completando, assim, a prova da afirmação.

Dessa forma, se definirmos $\psi_{n}: X \longrightarrow E_{n}$ tal que

$$
\psi_{n}(x)=\frac{\sigma_{n}\left(n \Delta_{n} \cdot x\right)}{\left\lceil\frac{n^{2} \Delta_{n}}{\delta_{n}}\right\rceil}
$$

em que, para cada $a \in \mathbb{R},\lceil a\rceil=\min \{j \in \mathbb{Z}: j \geq a\}$, teremos que, para quaisquer $x, y \in X$,

$$
\begin{aligned}
\|x-y\|_{X}<n & \Rightarrow\left\|n \Delta_{n} \cdot x-n \Delta_{n} \cdot y\right\|_{X}<n^{2} \Delta_{n}=\frac{n^{2} \Delta_{n}}{\delta_{n}} \cdot \delta_{n} \leq\left\lceil\frac{n^{2} \Delta_{n}}{\delta_{n}}\right\rceil \cdot \delta_{n} \\
& \Rightarrow\left\|\sigma_{n}\left(n \Delta_{n} \cdot x\right)-\sigma_{n}\left(n \Delta_{n} \cdot y\right)\right\|_{E}<\underbrace{\left[\frac{n^{2} \Delta_{n}}{\delta_{n}}\right\rceil}_{k \in \mathbb{N}} \cdot 2^{-n} \\
& \Rightarrow\left\|\psi_{n}(x)-\psi_{n}(y)\right\|_{E}<2^{-n}
\end{aligned}
$$

e, analogamente,

$$
\begin{aligned}
\|x-y\|_{X} \geq \frac{1}{n} & \Rightarrow\left\|n \Delta_{n} \cdot x-n \Delta_{n} \cdot y\right\|_{X} \geq \Delta_{n} \\
& \Rightarrow\left\|\sigma_{n}\left(n \Delta_{n} \cdot x\right)-\sigma_{n}\left(n \Delta_{n} \cdot y\right)\right\|_{E} \geq M_{n} \\
& \Rightarrow\left\|\psi_{n}(x)-\psi_{n}(y)\right\|_{E} \geq \frac{M_{n}}{\left\lceil\frac{n^{2} \Delta_{n}}{\delta_{n}}\right\rceil} .
\end{aligned}
$$

Voltemos, agora, a nossa atenção para a projeção $P_{n}$. Como, por hipótese, $P_{n}$ é limitada e não nula, sabemos que $\left\|P_{n}\right\|$ é finita e diferente de 0 . Logo, utilizando novamente a continuidade uniforme de $\sigma_{n}$, podemos fixar $\xi_{n}>0$ tal que, para todos $x, y \in X$,

$$
\|x-y\|_{X}<\xi_{n} \Rightarrow\left\|\sigma_{n}(x)-\sigma_{n}(y)\right\|_{E}<\frac{M_{n}}{n\left\|P_{n}\right\|} \cdot 2^{-n} .
$$

Assim sendo, se definirmos $\varphi_{n}: X \longrightarrow E_{n}$ de modo que

$$
\varphi_{n}(x)=\frac{n\left\|P_{n}\right\|}{M_{n}} \cdot \sigma_{n}\left(\frac{\xi_{n}}{n} \cdot x\right)
$$

teremos que, para todo $(x, y) \in X \times X$,

$$
\begin{aligned}
\|x-y\|_{X}<n & \Rightarrow\left\|\frac{\xi_{n}}{n} \cdot x-\frac{\xi_{n}}{n} \cdot y\right\|_{X}<\xi_{n} \\
& \Rightarrow\left\|\sigma_{n}\left(\frac{\xi_{n}}{n} \cdot x\right)-\sigma_{n}\left(\frac{\xi_{n}}{n} \cdot y\right)\right\|_{E}<\frac{M_{n}}{n\left\|P_{n}\right\|} \cdot 2^{-n} \\
& \Rightarrow\left\|\varphi_{n}(x)-\varphi_{n}(y)\right\|_{E}<2^{-n}
\end{aligned}
$$


e

$$
\begin{aligned}
\|x-y\|_{X} \geq \frac{n \Delta_{n}}{\xi_{n}} & \Rightarrow\left\|\frac{\xi_{n}}{n} \cdot x-\frac{\xi_{n}}{n} \cdot y\right\|_{X} \geq \Delta_{n} \\
& \Rightarrow\left\|\sigma_{n}\left(\frac{\xi}{n} \cdot x\right)-\sigma_{n}\left(\frac{\xi}{n} \cdot y\right)\right\|_{E} \geq M_{n} \\
& \Rightarrow\left\|\varphi_{n}(x)-\varphi_{n}(y)\right\|_{E} \geq n\left\|P_{n}\right\| .
\end{aligned}
$$

Observe, ainda, que $\psi_{n}$ e $\varphi_{n}$ são uniformemente contínuas. Com efeito, como $\sigma_{n}$ é uniformemente contínua, decorre diretamente do lema 5.3.1 que $\psi_{n}$ e $\varphi_{n}$ também o serão.

Repetindo essa construção para os demais valores de $n$, obtemos sequências $\left(\psi_{n}\right)_{n \in \mathbb{N}}$ e $\left(\varphi_{n}\right)_{n \in \mathbb{N}}$ de funções satisfazendo, respectivamente, às implicações (5.3)-(5.4) e (5.5)-(5.6). Nosso objetivo, agora, é construir a função $\omega$ a partir de $\left(\psi_{n}\right)_{n \in \mathbb{N}}$ e $\left(\varphi_{n}\right)_{n \in \mathbb{N}}$.

Para começar, tomemos $x, y \in X$ de modo arbitrário, e fixemos $m \in \mathbb{N}$ tal que $\|x-y\|_{X}<$ $m$. Note, em particular, que $\|x-y\|<n$ para todo $n \in \mathbb{N}$ tal que $n \geq m$. Sendo assim, como $n \leq 2 n-1 \leq 2 n$ para todo $n \in \mathbb{N}$, segue de (5.3) e de (5.5) que

$$
\left\|\psi_{2 n-1}(x)-\psi_{2 n-1}(y)\right\|_{E}<2^{-2 n+1}
$$

e

$$
\left\|\varphi_{2 n}(x)-\varphi_{2 n}(y)\right\|_{E}<2^{-2 n}
$$

para todo $n \in \mathbb{N}$ tal que $n \geq m$. Por sua vez, como $\sum_{n=1}^{+\infty} 2^{-2 n+1}$ e $\sum_{n=1}^{+\infty} 2^{-2 n}$ convergem, segue do Critério da comparação que $\sum_{n=1}^{+\infty}\left\|\psi_{2 n-1}(x)-\psi_{2 n-1}(y)\right\|_{E}$ e $\sum_{n=1}^{+\infty}\left\|\varphi_{2 n}(x)-\varphi_{2 n}(y)\right\|_{E}$ convergem. Mas, mais do que isso, o Critério da comparação nos fornece cotas superiores para $\sum_{n=1}^{+\infty}\left\|\psi_{2 n-1}(x)-\psi_{2 n-1}(y)\right\|_{E}$ e $\sum_{n=1}^{+\infty}\left\|\varphi_{2 n}(x)-\varphi_{2 n}(y)\right\|_{E}$. Ele nos diz que

$$
\sum_{n=m}^{+\infty}\left\|\psi_{2 n-1}(x)-\psi_{2 n-1}(y)\right\|_{E} \leq \sum_{n=m}^{+\infty} 2^{-2 n+1}=2 \sum_{n=m}^{+\infty}\left(\frac{1}{4}\right)^{n}=2 \cdot \frac{\frac{1}{4^{m}}}{1-\frac{1}{4}}=\frac{1}{3} \cdot 2^{-2 m+3}
$$

e que

$$
\sum_{n=m}^{+\infty}\left\|\varphi_{2 n}(x)-\varphi_{2 n}(y)\right\|_{E} \leq \sum_{n=m}^{+\infty} 2^{-2 n}=\sum_{n=m}^{+\infty}\left(\frac{1}{4}\right)^{n}=\frac{1}{3} \cdot 2^{-2 m+2} .
$$

Isso pode não parecer muito útil agora, mas será de grande importância em etapas posteriores da prova.

Voltemos, por hora, a nos concentrar na convergência das séries em questão. Tomando $y=0_{X}$ e notando que $\psi_{n}\left(0_{X}\right)=\varphi_{n}\left(0_{X}\right)=0_{E}$ para todo $n \in \mathbb{N}$ (pois, por hipótese, $\sigma_{n}\left(0_{X}\right)=0_{E}$ para cada $n \in \mathbb{N})$, concluímos que $\sum_{n=1}^{+\infty} \psi_{2 n-1}(x)$ e $\sum_{n=1}^{+\infty} \varphi_{2 n}(x)$ são absolutamente convergentes em $\left(E,\|\cdot\|_{E}\right)$. Como $\left(E,\|\cdot\|_{E}\right)$ é um espaço de Banach, disso resulta que $\sum_{n=1}^{+\infty} \psi_{2 n-1}(x)$ e $\sum_{n=1}^{+\infty} \varphi_{2 n}(x)$ convergem em $\left(E,\|\cdot\|_{E}\right)$. E, como $x \in X$ arbitrário, isso mostra que a função $\omega: X \stackrel{n=1}{\longrightarrow}$ tal 
que

$$
\omega(x)=\sum_{n=1}^{+\infty} \psi_{2 n-1}(x)+\sum_{n=1}^{+\infty} \varphi_{2 n}(x)
$$

está bem definida. Resta mostrarmos que $\omega$ é um mergulho uniforme e um mergulho grosseiro. Para tanto, vamos começar mostrando que $\omega$ é uniformemente contínua. Como $\left(X,\|\cdot\|_{X}\right)$ é um espaço normado, disso seguirá, automaticamente, que $\omega$ é bornológica (veja o teorema 4.4.6).

Seja $\varepsilon>0$ dado. Como $\lim _{m \rightarrow+\infty} 2^{-2 m+3}=0$, podemos fixar $m \in\{2,3, \ldots\}$ tal que $2^{-2 m+3}<\varepsilon$. E, uma vez que $\psi_{n}$ e $\varphi_{n}$ são uniformemente contínuas para todo $n \in \mathbb{N}, \sum_{n=1}^{m-1}\left(\psi_{2 n-1}+\varphi_{2 n}\right)$ é, também, uniformemente contínua. Assim sendo, podemos tomar $\eta>0$ tal que

$$
\left\|\sum_{n=1}^{m-1}\left(\psi_{2 n-1}(x)+\varphi_{2 n}(x)\right)-\sum_{n=1}^{m-1}\left(\psi_{2 n-1}(y)+\varphi_{2 n}(y)\right)\right\|_{E}<\frac{\varepsilon}{3}
$$

para todos $x, y \in X$ tais que $\|x-y\|_{X}<\eta$.

Por outro lado, se $x, y \in X$ forem tais que $\|x-y\|_{X}<m$, segue de (5.7) e (5.8), da continuidade de $\|\cdot\|_{E}$ e da desigualdade triangular que

$$
\left\|\sum_{n=m}^{+\infty}\left(\psi_{2 n-1}(x)-\psi_{2 n-1}(y)\right)\right\|_{E} \leq \sum_{n=m}^{+\infty}\left\|\psi_{2 n-1}(x)-\psi_{2 n-1}(y)\right\|_{E} \leq \frac{1}{3} \cdot 2^{-2 m+3}<\frac{\varepsilon}{3}
$$

e

$$
\left\|\sum_{n=m}^{+\infty}\left(\varphi_{2 n}(x)-\varphi_{2 n}(y)\right)\right\|_{E} \leq \sum_{n=m}^{+\infty}\left\|\varphi_{2 n}(x)-\varphi_{2 n}(y)\right\| \leq \frac{1}{3} \cdot 2^{-2 m+2}=\frac{1}{6} \cdot 2^{2 m+3}<\frac{\varepsilon}{6} .
$$

Dessa forma, tomando $\delta:=\min \{\eta, m\}$, teremos que

$$
\begin{aligned}
\|\omega(x)-\omega(y)\|_{E} & =\left\|\sum_{n=1}^{+\infty}\left(\psi_{2 n-1}(x)+\varphi_{2 n}(x)\right)-\sum_{n=1}^{+\infty}\left(\psi_{2 n-1}(y)+\varphi_{2 n}(y)\right)\right\|_{E} \\
& \leq\left\|\sum_{n=1}^{m-1}\left(\psi_{2 n-1}(x)+\varphi_{2 n}(x)\right)-\sum_{n=1}^{m-1}\left(\psi_{2 n-1}(y)+\varphi_{2 n}(y)\right)\right\|_{E} \\
& +\left\|\sum_{n=m}^{+\infty}\left(\psi_{2 n-1}(x)-\psi_{2 n-1}(y)\right)\right\|_{E}+\left\|\sum_{n=m}^{+\infty}\left(\varphi_{2 n}(x)-\varphi_{2 n}(y)\right)\right\|_{E} \\
& <\frac{\varepsilon}{3}+\frac{\varepsilon}{3}+\frac{\varepsilon}{6}<\varepsilon
\end{aligned}
$$

para todos $x, y \in X$ tais que $\|x-y\|_{X}<\delta$. Como $\varepsilon>0$ é arbitrário, disso concluímos que $\omega$ é uniformemente contínua (e, portanto, bornológica).

Estamos, agora, na última etapa da prova. Precisamos, ainda, mostrar que $\omega$ é um mergulho uniforme expansível. Para isso, utilizaremos a sequência $\left(P_{n}\right)_{n \in \mathbb{N}}$ de projeções limitadas. Inicialmente, note que, dados $m \in \mathbb{N}$ e $x, y \in X$, temos que

$$
\left\|P_{m}(\omega(x))-P_{m}(\omega(y))\right\|_{E}=\left\|P_{m}(\omega(x)-\omega(y))\right\|_{E} \leq\left\|P_{m}\right\| \cdot\|\omega(x)-\omega(y)\|_{E} .
$$

Observe ainda que $E_{n} \subseteq \operatorname{Ker}\left(P_{m}\right)$ para todo $n \in \mathbb{N}$ tal que $n \neq m$. Além disso, como $P_{m}$ é uma projeção sobre $E_{m}$, temos que $P_{m}(v)=v$ para todo $v \in E_{m}$. E, uma vez que $\operatorname{Im}\left(\psi_{m}\right), \operatorname{Im}\left(\varphi_{m}\right) \subseteq$ 
$E_{m}$, isso implica que, para todo $x \in X$,

$$
P_{m}\left(\psi_{m}(x)\right)=\psi_{m}(x) \text { e } P_{m}\left(\varphi_{m}(x)\right)=\varphi_{m}(x) .
$$

Tendo isso em mente, é fácil ver que. para todo $n \in \mathbb{N}$ e todo $x \in X$,

$$
\begin{aligned}
P_{2 n-1}(\omega(x)) & =P_{2 n-1}\left(\sum_{j=1}^{+\infty}\left[\psi_{2 j-1}(x)+\varphi_{2 j}(x)\right]\right)=\sum_{j=1}^{+\infty} P_{2 n-1}\left(\psi_{2 j-1}(x)+\varphi_{2 j}(x)\right) \\
& =\sum_{j=1}^{+\infty}\left[P_{2 n-1}\left(\psi_{2 j-1}(x)\right)+P_{2 n-1}\left(\varphi_{2 j}(x)\right)\right]=\psi_{2 n-1}(x)
\end{aligned}
$$

e, analogamente,

$$
P_{2 n}(\omega(x))=P_{2 n}\left(\sum_{j=1}^{+\infty}\left[\psi_{2 j-1}(x)+\varphi_{2 j}(x)\right]\right)=\sum_{j=1}^{+\infty}\left[P_{2 n}\left(\psi_{2 j-1}(x)\right)+P_{2 n}\left(\varphi_{2 j}(x)\right)\right]=\varphi_{2 n}(x) .
$$

(Note que utilizamos a continuidade de $P_{2 n-1}$ e de $P_{2 n}$, respectivamente, para permutar o operador e o símbolo de somatório na segunda igualdade das equações (5.10) e (5.11).)

Podemos, agora, concluir a prova de que $\omega$ é um mergulho uniforme. Para isso, fixemos um $\Delta>0$ qualquer, e tomemos $n \in \mathbb{N}$ tal que $\frac{1}{2 n-1} \leq \Delta$. Nessas condições, se $x, y \in X$ forem tais que $\|x-y\|_{X} \geq \Delta \geq \frac{1}{2 n-1}$, então, por (5.4), (5.9) e (5.10), teremos que

$$
\begin{aligned}
\|\omega(x)-\omega(y)\|_{E} & \geq \frac{1}{\left\|P_{2 n-1}\right\|} \cdot\left\|P_{2 n-1}(\omega(x))-P_{2 n-1}(\omega(y))\right\|_{E} \\
& =\frac{1}{\left\|P_{2 n-1}\right\|} \cdot\left\|\psi_{2 n-1}(x)-\psi_{2 n-1}(y)\right\|_{E} \\
& \geq \frac{1}{\left\|P_{2 n-1}\right\|} \cdot \frac{M_{2 n-1}}{\left\lceil\frac{(2 n-1)^{2} \Delta_{2 n-1}}{\delta_{2 n-1}}\right\rceil} .
\end{aligned}
$$

Como $\Delta>0$ é arbitrário, disso resulta que $\omega$ é um mergulho uniforme.

Por fim, vamos mostrar que $\omega$ é expansível. Como já sabemos que $\omega$ é bornológica, disso seguirá que $\omega$ é um megulho coarse, completando a demonstração.

Seja $M>0$ dado, e tomemos $n \in \mathbb{N}$ tal que $n \geq \frac{M}{2}$. Nessas condições, segue de (5.9), (5.11) e (5.6) que, para todos $x, y \in X$ tais que $\|x-y\|_{X} \geq \frac{2 n \Delta_{2 n}}{\xi_{2 n}}$, vale que

$$
\begin{aligned}
\|\omega(x)-\omega(y)\|_{E} & \geq \frac{1}{\left\|P_{2 n}\right\|} \cdot\left\|P_{2 n}(\omega(x))-P_{2 n}(\omega(y))\right\|_{E} \\
& =\frac{1}{\left\|P_{2 n}\right\|} \cdot\left\|\varphi_{2 n}(x)-\varphi_{2 n}(y)\right\|_{E} \geq 2 n \geq M
\end{aligned}
$$

E, como $M>0$ é arbitrário, isso prova que $\omega$ é expansível e conclui a demonstração do teorema.

O teorema 5.3.2 é muito interessante. Em parte por se tratar de um resultado poderoso e com ampla aplicabilidade. E, em parte, pelo fato de que a sua demonstração se apoia inteiramente 
em técnicas e resultados elementares utilizados de forma bastante engenhosa. O teorema 5.3.3, a seguir, é um de seus corolários.

TeOrema 5.3.3. Sejam $\left(X,\|\cdot\|_{X}\right)$ e $\left(E,\|\cdot\|_{E}\right)$ espaços de Banach, e $\sigma: X \longrightarrow$ E uma função não colapsada e uniformemente contínua. Nessas condições, para cada $p \in[1,+\infty[$, existe $\omega_{p}: X \longrightarrow \ell_{p}(E)$ tal que $\omega_{p}$ é um mergulho uniforme e também um mergulho grosseiro.

Demonstração. Seja $p \in\left[1,+\infty\left[\right.\right.$ dado. Como $\left(E,\|\cdot\|_{X}\right)$ é, por hipótese, um espaço de Banach, segue da proposição 5.2.3 que $\left(\ell_{p}(E),\|\cdot\|_{p}\right)$ é, também, um espaço de Banach. Dessa forma, se contruírmos sequências $\left(P_{n}\right)_{n \in \mathbb{N}}$ e $\left(\sigma_{n}\right)_{n \in \mathbb{N}}$ de funções satisfazendo as hipóteses do teorema 5.3.2, seguirá de 5.3 .2 que existe $\omega_{p}: X \longrightarrow \ell_{p}(E)$ nas condições do enunciado. Vamos, então, construir tais sequências.

Para cada $n \in \mathbb{N}$, sejam $E_{n}$ o subespaço de $\ell_{p}(E)$ gerado por $\left\{\left(x \delta_{j n}\right)_{j \in \mathbb{N}}: x \in E\right\}$,

$$
\begin{aligned}
P_{n}: \ell_{p}(E) & \longrightarrow \ell_{p}(E) \\
\left(x_{j}\right)_{j \in \mathbb{N}} & \longmapsto\left(x_{n} \delta_{j n}\right)_{j \in \mathbb{N}}
\end{aligned}
$$

e

$$
\begin{aligned}
\sigma_{n}: X & \longrightarrow E_{n} \\
x & \longmapsto\left(\sigma(x) \delta_{j n}\right)_{j \in \mathbb{N}} .
\end{aligned}
$$

Dados $n \in \mathbb{N}, x=\left(x_{j}\right)_{j \in \mathbb{N}}$ e $y=\left(y_{j}\right)_{j \in \mathbb{N}}$ em $\ell_{p}(E)$, e $\lambda \in \mathbb{K}$, é imediato ver que

$$
\begin{aligned}
P_{n}(x+\lambda y) & =P_{n}\left(\left(x_{j}+\lambda y_{j}\right)_{j \in \mathbb{N}}\right)=\left(\left(x_{n}+\lambda y_{n}\right) \delta_{j n}\right)_{j \in \mathbb{N}}=\left(x_{n} \delta_{j n}\right)_{j \in \mathbb{N}}+\lambda\left(y_{n} \delta_{j n}\right)_{j \in \mathbb{N}} \\
& =P_{n}(x)+\lambda P_{n}(y) .
\end{aligned}
$$

Isso mostra que $P_{n}$ é linear. Note ainda que $P_{n}(x)=x$ para todo $x \in E_{n}$. Como $\operatorname{Im}\left(P_{n}\right) \subseteq E_{n}$, disso concluímos que $P_{n}$ é uma projeção sobre $E_{n}$. E também é fácil ver que $E_{m} \subseteq \operatorname{Ker}\left(P_{n}\right)$ para todo $m \in \mathbb{N}$ tal que $m \neq n$.

Por fim, observe que, para cada $x:=\left(x_{j}\right)_{j \in \mathbb{N}}$ em $\ell_{p}(E)$, vale que

$$
\left\|P_{n}(x)\right\|_{p}=\left\|\left(x_{n} \delta_{j n}\right)_{j \in \mathbb{N}}\right\|_{p}=\left(\sum_{j=1}^{+\infty}\left\|x_{n} \delta_{j n}\right\|_{E}^{p}\right)^{\frac{1}{p}}=\left\|x_{n}\right\|_{E} \leq\left(\sum_{j=1}^{+\infty}\left\|x_{j}\right\|_{E}^{p}\right)^{\frac{1}{p}}=\|x\|_{p},
$$

a partir do que segue, imediatamente, que $P_{n}$ é limitada (e que $\left\|P_{n}\right\| \leq 1 .^{4}$ )

Façamos, agora, algumas considerações a respeito de $\sigma_{n}$. Inicialmente, observe que $\sigma_{n}$ está bem definida e que, para todos $x, y \in X$,

$$
\begin{aligned}
\left\|\sigma_{n}(x)-\sigma_{n}(y)\right\|_{p} & =\left\|\left((\sigma(x)-\sigma(y)) \delta_{j n}\right)_{j \in \mathbb{N}}\right\|_{p}=\left(\sum_{j=1}^{+\infty}\|\sigma(x)-\sigma(y)\|_{E}^{p} \delta_{j n}\right)^{\frac{1}{p}} \\
& =\|\sigma(x)-\sigma(y)\|_{E} .
\end{aligned}
$$

Logo, como $\sigma$ é uniformemente contínua e não colapsada, $\sigma_{n}$ também o será. E, uma vez que $P_{n}$ e $\sigma_{n}$ satisfazem as hipóteses do teorema 5.3.2 para um $n \in \mathbb{N}$ arbitrário, podemos aplicá-lo às sequências $\left(P_{n}\right)_{n \in \mathbb{N}}$ e $\left(\sigma_{n}\right)_{n \in \mathbb{N}}$ e concluir a demonstração.

\footnotetext{
${ }^{4}$ Em verdade, é fácil ver que $\left\|P_{n}\right\|=1$ (pois $\left\|P_{n}\right\| \leq 1$ e $\left\|P_{n}\left(\left(x \delta_{j n}\right)_{j \in \mathbb{N}}\right)\right\|_{p}=1=\left\|\left(x \delta_{j n}\right)_{j \in \mathbb{N}}\right\|_{p}$ para todo $x \in E$ tal que $\|x\|_{E}=1$ ).
} 
Veremos, a seguir, uma aplicação do teorema 5.3.3. Mais especificamente, vamos mostrar que, dados um espaço de Banach $\left(X,\|\cdot\|_{X}\right)$ e um real $p \in[1,+\infty[$, se existir um mergulho uniforme de $\left(X,\|\cdot\|_{X}\right)$ em $\left(\ell_{p},|\cdot|_{p}\right)$, então existirá, também, um mergulho grosseiro. Para isso, precisaremos, inicialmente, demonstrar um lema auxiliar, o qual faz uso do resultado bem conhecido de que $\mathbb{N}$ e $\mathbb{N}^{2}$ possuem a mesma cardinalidade.

LEMA 5.3.4. Sejam $\sigma:=\left(\sigma_{1}, \sigma_{2}\right): \mathbb{N} \longrightarrow \mathbb{N}^{2}$ uma bijeção, $p \in[1,+\infty[$ um real dado, $e$ $\left(\left(x_{m}^{j}\right)_{j \in \mathbb{N}}\right)_{m \in \mathbb{N}}$ uma sequência de sequências tal que, para cada $(m, j) \in \mathbb{N}^{2}, x_{m}^{j} \in \mathbb{K}$. Nessas condições, se $\sum_{m=1}^{+\infty} \sum_{j=1}^{+\infty}\left|x_{m}^{j}\right|^{p}$ convergir, então $\sum_{n=1}^{+\infty}\left|x_{\sigma_{1}(n)}^{\sigma_{2}(n)}\right|^{p}$ converge, e, nesse caso, ambas as séries têm a mesma soma.

Demonstração. Inicialmente, suponhamos que $\sum_{m=1}^{+\infty} \sum_{j=1}^{+\infty}\left|x_{m}^{j}\right|^{p}$ convirja, e indiquemos por $s_{1} \mathrm{o}$ valor de sua soma. Vamos, agora, provar que $\sum_{n=1}^{+\infty}\left|x_{\sigma_{1}(n)}^{\sigma_{2}(n)}\right|^{p}$ converge, e que

$$
\sum_{n=1}^{+\infty}\left|x_{\sigma_{1}(n)}^{\sigma_{2}(n)}\right|^{p}=s_{1}
$$

Para isso, tomemos $n_{0} \in \mathbb{N}$ de modo arbitrário. Observe que cada termo de $\sum_{n=1}^{n_{0}}\left|x_{\sigma_{1}(n)}^{\sigma_{2}(n)}\right|^{p}$ pertence a $\left\{x_{m}^{j}: m, j \in \mathbb{N}\right\}$. Além disso, como $\sigma$ é injetora, cada termo de $\sum_{m=1}^{+\infty} \sum_{j=1}^{+\infty}\left|x_{m}^{j}\right|^{p}$ aparece em $\sum_{n=1}^{n_{0}}\left|x_{\sigma_{1}(n)}^{\sigma_{2}(n)}\right|^{p}$ no máximo uma vez. Dessa forma,

$$
\sum_{n=1}^{n_{0}}\left|x_{\sigma_{1}(n)}^{\sigma_{2}(n)}\right|^{p} \leq \sum_{m=1}^{+\infty} \sum_{j=1}^{+\infty}\left|x_{m}^{j}\right|^{p}=s_{1}
$$

Como $n_{0} \in \mathbb{N}$ é arbitrário, isso mostra que $\sum_{n=1}^{+\infty}\left|x_{\sigma_{1}(n)}^{\sigma_{2}(n)}\right|^{p}$ converge, e que

$$
s_{2}:=\sum_{n=1}^{+\infty}\left|x_{\sigma_{1}(n)}^{\sigma_{2}(n)}\right|^{p} \leq s_{1}
$$

(veja o corolário 2.4.2).

Tomemos, também, $m_{0} \in \mathbb{N}$ qualquer, e notemos que, sendo $\sigma$ uma função sobrejetora, cada termo de $\sum_{m=1}^{m_{0}} \sum_{j=1}^{+\infty}\left|x_{m}^{j}\right|^{p}$ pertence a $\left\{x_{\sigma_{1}(n)}^{\sigma_{2}(n)}: n \in \mathbb{N}\right\}$. Assim sendo,

$$
\sum_{m=1}^{m_{0}} \sum_{j=1}^{+\infty}\left|x_{m}^{j}\right|^{p} \leq \sum_{n=1}^{+\infty}\left|x_{\sigma_{1}(n)}^{\sigma_{2}(n)}\right|^{p}=s_{2}
$$


E, como $m_{0} \in \mathbb{N}$ é qualquer, disso resulta que

$$
s_{1}=\lim _{m_{0} \rightarrow+\infty}\left[\sum_{m=1}^{m_{0}} \sum_{j=1}^{+\infty}\left|x_{m}^{j}\right|^{p}\right] \leq s_{2}
$$

a partir do que concluímos que $s_{1}=s_{2}$.

Podemos, agora, provar o seguinte corolário do teorema 5.3.3:

Corolário 5.3.5. Sejam $\left(X,\|\cdot\|_{X}\right)$ um espaço de Banach e $p \in[1,+\infty[$ um real dado, $e$ suponhamos que exista um mergulho uniforme de $\left(X,\|\cdot\|_{X}\right)$ em $\left(\ell_{p},|\cdot|_{p}\right)$. Nessas condições, existe $\Psi: X \longrightarrow \ell_{p}$ tal que $\Psi$ é um mergulho uniforme e também um mergulho grosseiro.

Demonstração. Seja $\varphi$ um mergulho uniforme de $\left(X,\|\cdot\|_{X}\right)$ em $\left(\ell_{p},|\cdot|_{p}\right)$. Inicialmente, observe que $\left(\ell_{p},|\cdot|_{p}\right)$ é um espaço de Banach e que $\varphi$ é, trivialmente, não colapsado. Assim sendo, segue do teorema 5.3 .3 que existe $\omega: X \longrightarrow \ell_{p}\left(\ell_{p},|\cdot|_{p}\right)$ tal que $\omega$ é, ao mesmo tempo, um mergulho uniforme e um mergulho grosseiro.

Consideremos, agora, a função

$$
\begin{aligned}
& \Phi: \ell_{p}\left(\ell_{p},|\cdot|_{p}\right) \longrightarrow \ell_{p} \\
& \left(\left(x_{m}^{j}\right)_{j \in \mathbb{N}}\right)_{m \in \mathbb{N}} \longmapsto\left(x_{\sigma_{1}(n)}^{\sigma_{2}(n)}\right)_{n \in \mathbb{N}^{*}}
\end{aligned}
$$

Note que, pelo lema 5.3.4, $\Phi$ está bem definida. E, mais do que isso, o lema mostra que $\Phi$ é uma isometria. Com efeito, dado $x=\left(\left(x_{m}^{j}\right)_{j \in \mathbb{N}}\right)_{m \in \mathbb{N}} \in \ell_{p}\left(\ell_{p},|\cdot|_{p}\right)$, segue de 5.3.4 que

$$
|\Phi(x)|_{p}=\left(\sum_{n=1}^{+\infty}\left|x_{\sigma_{1}(n)}^{\sigma_{2}(n)}\right|^{p}\right)^{\frac{1}{p}}=\left(\sum_{m=1}^{+\infty} \sum_{j=1}^{+\infty}\left|x_{m}^{j}\right|^{p}\right)^{\frac{1}{p}}=\left(\sum_{m=1}^{+\infty}\left|\left(x_{m}^{j}\right)_{j \in \mathbb{N}}\right|_{p}^{p}\right)^{\frac{1}{p}}=\|x\|_{p} .
$$

Por fim, observe ainda que $\Phi$ é, trivialmente, linear. Assim sendo, para todos $x, y \in X$, temos que

$$
|(\Phi \circ \omega)(x)-(\Phi \circ \omega)(y)|_{p}=|\Phi(\omega(x))-\Phi(\omega(y))|_{p}=|\Phi(\omega(x)-\omega(y))|_{p}=\|\omega(x)-\omega(y)\|_{p} .
$$

E, como $\omega$ é um mergulho uniforme e um mergulho grosseiro, disso concluímos que $\Phi \circ \omega$ : $X \longrightarrow \ell_{p}$ é, também, um mergulho uniforme e um mergulho grosseiro.

Observe que o corolário 5.3.5 responde afirmativamente a QUESTÃo 2 no caso particular em que $E=\ell_{p}$ para algum $p \in[1,+\infty[$. E mais: ele nos diz que, nesse caso, existe um mergulho grosseiro de $\left(X,\|\cdot\|_{X}\right)$ em $\left(E,\|\cdot\|_{E}\right)$ o qual é, também, um mergulho uniforme.

Após refletirmos um pouco a respeito do teorema 5.3.3, é natural nos perguntarmos por quais espaços normados podemos substituir $\left(\ell_{p}(E),\|\cdot\|_{p}\right)$ no enunciado do teorema 5.3 .3 de modo que a conclusão continue válida. O próximo teorema mostra que, sob hipóteses um pouco mais fortes, isso vale, ao menos em parte, para o espaço $\left(E \times E,\|\cdot\|_{E \times E}\right)$. E, uma vez que, na maioria das vezes, é mais fácil trabalharmos com $\left(E \times E,\|\cdot\|_{E \times E}\right)$ em detrimento de $\left(\ell_{p}(E),\|\cdot\|_{p}\right)$, esse resultado mostra-se particularmente útil em muitos casos de interesse, nos quais a passagem de $\left(E,\|\cdot\|_{E}\right)$ a $\left(\ell_{p}(E),\|\cdot\|_{p}\right)$ é, por demais, problemática.

TeOrema 5.3.6. Sejam $\left(X,\|\cdot\|_{X}\right)$ e $\left(E,\|\cdot\|_{E}\right)$ espaços de Banach, e $\sigma: X \longrightarrow$ uma função limitada, não colapsada e uniformemente continua. Nessas condições, existe um mergulho grosseiro uniformemente contínuo de $\left(X,\|\cdot\|_{X}\right)$ em $\left(E \times E,\|\cdot\|_{E \times E}\right)$. 
O teorema 5.3.6 é uma consequência direta de um resultado mais geral, o qual enunciaremos a seguir.

TeOrema 5.3.7. Sejam $\left(X,\|\cdot\|_{X}\right),\left(E,\|\cdot\|_{E}\right)$ e $\left(F,\|\cdot\|_{F}\right)$ espaços de Banach, e $\sigma: X \longrightarrow E$, $\omega: X \longrightarrow F$ funções limitadas, não colapsadas e uniformemente continuas. Nessas condições, existe um mergulho grosseiro $\Phi: X \longrightarrow E \times F$ que é, também, uniformemente contínuo.

OBSERVAÇÃo 5.3.8. Observe que o teorema 5.3.7 se reduz ao teorema 5.3.6 no caso particular em que $E=F$ e $\omega=\sigma$.

Por uma questão de organização, dividiremos a demonstração do teorema 5.3.7 em duas etapas. Primeiramente, provaremos um lema auxiliar e, em seguida, utilizaremos o lema para demonstrar o teorema.

LEMA 5.3.9. Sejam $\left(X,\|\cdot\|_{X}\right),\left(E,\|\cdot\|_{E}\right)$ e $\left(F,\|\cdot\|_{F}\right)$ espaços de Banach, e $\sigma: X \longrightarrow E$, $\omega: X \longrightarrow F$ funções limitadas, não colapsadas e uniformemente contínuas. Nessas condições, podemos construir sequências $\left(r_{n}\right)_{n \in \mathbb{N}} e\left(t_{n}\right)_{n \in \mathbb{N}_{0}}$ de modo que

$$
0=t_{0}<r_{1}<t_{1}<r_{2}<t_{2}<\ldots
$$

$e \lim _{n \rightarrow+\infty} r_{n}=\lim _{n \rightarrow+\infty} t_{n}=+\infty$, e, conjuntamente, definir sequências de funções, $\left(\varphi_{n}\right)_{n \in \mathbb{N}} e$ $\left(\psi_{n}\right)_{n \in \mathbb{N}}$, de forma que $\varphi_{n}: X \longrightarrow E$ e $\psi_{n}: X \longrightarrow F$ sejam limitadas, uniformemente contínuas e tais que $\varphi_{n}\left(0_{X}\right)=0_{E}$ e $\psi_{n}\left(0_{X}\right)=0_{F}$ para cada $n \in \mathbb{N}$ e, para todo $n \in \mathbb{N}$ e todos $x, y \in X$, tenhamos que

$$
\begin{gathered}
\|x-y\|_{X} \geq r_{n} \Rightarrow\left\|\sum_{i=1}^{n}\left(\varphi_{i}(x)-\varphi_{i}(y)\right)\right\|_{E} \geq 2^{n}, \\
\|x-y\|_{X} \geq t_{n} \Rightarrow\left\|\sum_{i=1}^{n}\left(\psi_{i}(x)-\psi_{i}(y)\right)\right\|_{F} \geq 2^{n}, \\
\|x-y\|_{X} \leq t_{n-1} \Rightarrow\left\|\varphi_{n}(x)-\varphi_{n}(y)\right\|_{E} \leq 2^{-n}
\end{gathered}
$$

$e$

$$
\|x-y\|_{X} \leq r_{n} \Rightarrow\left\|\psi_{n}(x)-\psi_{n}(y)\right\|_{F} \leq 2^{-n} .
$$

Demonstração. Como $\sigma$ e $\omega$ são não colapsadas, existem $\Delta, M>0$ tais que, para todos $x, y \in$ $X$,

$$
\|x-y\|_{X} \geq \Delta \Rightarrow\left(\|\sigma(x)-\sigma(y)\|_{E} \geq M \text { e }\|\omega(x)-\omega(y)\|_{F} \geq M\right) .
$$

Observe que podemos supor, sem perda de generalidade, que $\Delta \geq 2$. Além disso, como $\sigma^{\prime}$ : $X \longrightarrow E$ e $\omega^{\prime}: X \longrightarrow F$ tais que

$$
\sigma^{\prime}(x)=\sigma(x)-\sigma\left(0_{X}\right) \text { e } \omega^{\prime}(x)=\omega(x)-\omega\left(0_{X}\right)
$$

estão bem definidas, são não colapsadas, limitadas, uniformemente contínuas e tais que $\sigma^{\prime}\left(0_{X}\right)=$ $0_{E}$ e $\omega^{\prime}\left(0_{X}\right)=0_{F}$, podemos, também, assumir que $\sigma\left(0_{X}\right)=0_{E}$ e que $\omega\left(0_{X}\right)=0_{F}$.

Vamos construir as sequências por recursão. Para começar, definamos $t_{0}:=0, r_{1}:=\Delta \mathrm{e}$ $\varphi_{1}:=\frac{2}{M} \cdot \sigma$. Como $\sigma$ é limitada e uniformemente contínua, $\varphi_{1}$ também o será (veja o lema 5.3.1). Além disso, está claro que $\varphi_{1}\left(0_{X}\right)=0_{E}$ e que $t_{0}=0<\Delta=r_{1}$. E também não é difícil ver que, para todos $x, y \in X$,

$$
\|x-y\|_{X} \geq r_{1}=\Delta \Rightarrow\|\sigma(x)-\sigma(y)\|_{E} \geq M \Rightarrow\left\|\varphi_{1}(x)-\varphi_{1}(y)\right\|_{E} \geq 2
$$


e

$$
\|x-y\|_{X} \leq t_{0}=0 \Rightarrow x=y \Rightarrow\left\|\varphi_{1}(x)-\varphi_{1}(y)\right\|_{E}=0<2^{-1} .
$$

Como próximo passo, vamos construir $\psi_{1}$ e $t_{1}$ a partir de $r_{1}$. Para isso, utilizaremos a continuidade uniforme de $\omega$. Comecemos fixando $\left.\delta_{1} \in\right] 0,1\left[\right.$ tal que $\|\omega(x)-\omega(y)\|_{F}<\frac{M}{4}$ para todos $x, y \in X$ tais que $\|x-y\|_{X} \leq \delta_{1}$. Em seguida, tomemos $t_{1}:=\frac{\Delta^{2}}{\delta_{1}}$ e definamos $\psi_{1}$ : $X \longrightarrow F$ de modo que $\psi_{1}(x)=\frac{2}{M} \cdot \omega\left(\frac{\delta_{1}}{\Delta} \cdot x\right)$. Inicialmente, note que $\psi_{1}$ é limitada e uniformemente contínua, uma vez que, por hipótese, $\omega$ é limitada e uniformemente contínua. Também é fácil ver que $\psi_{1}\left(0_{X}\right)=0_{F}$ e que, para todos $x, y \in X$,

$$
\begin{aligned}
\|x-y\|_{X} \geq t_{1}=\frac{\Delta^{2}}{\delta_{1}} & \Rightarrow\left\|\frac{\delta_{1}}{\Delta} \cdot x-\frac{\delta_{1}}{\Delta} \cdot y\right\|_{X} \geq \Delta \Rightarrow\left\|\omega\left(\frac{\delta_{1}}{\Delta} \cdot x\right)-\omega\left(\frac{\delta_{1}}{\Delta} \cdot y\right)\right\|_{F} \geq M \\
& \Rightarrow\left\|\psi_{1}(x)-\psi_{1}(y)\right\|_{F} \geq 2 .
\end{aligned}
$$

e

$$
\begin{aligned}
\|x-y\|_{X} \leq r_{1}=\Delta & \Rightarrow\left\|\frac{\delta_{1}}{\Delta} \cdot x-\frac{\delta_{1}}{\Delta} \cdot y\right\|_{X} \leq \delta_{1} \Rightarrow\left\|\omega\left(\frac{\delta_{1}}{\Delta} \cdot x\right)-\omega\left(\frac{\delta_{1}}{\Delta} \cdot y\right)\right\|_{F}<\frac{M}{4} \\
& \Rightarrow\left\|\psi_{1}(x)-\psi_{1}(y)\right\|_{F}<2^{-1} .
\end{aligned}
$$

Por fim, observe que, de $\delta_{1}<1$ e $\Delta \geq 2$, segue que $t_{1}=\frac{\Delta^{2}}{\delta_{1}}=\underbrace{\frac{\Delta}{\delta_{1}}}_{>2} \cdot \underbrace{\Delta}_{=r_{1}}>2 r_{1}>r_{1}$. Note a presença do 2 nessa desigualdade. Isso será útil mais adiante.

A seguir, mostraremos como, dado $n \in \mathbb{N}$, podemos construir $r_{n+1}$ e $\varphi_{n+1}$ a partir de $\varphi_{1}, \ldots, \varphi_{n}$ e $0=t_{0}<r_{1}<\ldots<t_{n}$. Para isso, utilizaremos, fortemente, a continuidade uniforme de $\sigma$.

Inicialmente, consideremos o conjunto

$$
A_{n+1}:=\left\{\left\|\sum_{i=1}^{n}\left(\varphi_{i}(x)-\varphi_{i}(y)\right)\right\|_{E}: x, y \in X\right\}
$$

Note que $A_{n+1}$ é não vazio e limitado superiormente (uma vez que, por construção, $\varphi_{1}, \ldots, \varphi_{n}$ são limitadas) e, portanto, tem supremo, o qual denotaremos por $S_{n+1}$.

Em seguida, fixemos $\left.\alpha_{n+1} \in\right] 0,1[$ tal que

$$
\|\sigma(x)-\sigma(y)\|_{E}<\frac{M}{S_{n+1}+2^{n+1}} \cdot 2^{-(n+1)}
$$

para todos $x, y \in X$ tais que $\|x-y\|_{X} \leq \alpha_{n+1}$, tomemos $r_{n+1}:=\frac{\Delta t_{n}}{\alpha_{n+1}}$ e definamos

$$
\varphi_{n+1}: X \longrightarrow E
$$

de modo que

$$
\varphi_{n+1}(x)=\frac{S_{n+1}+2^{n+1}}{M} \cdot \sigma\left(\frac{\alpha_{n+1}}{t_{n}} \cdot x\right)
$$


Observe que $\varphi_{n+1}$ é limitada e uniformemente contínua (uma vez que $\sigma$ também o é) e que

$$
r_{n+1}=\underbrace{\frac{\Delta}{\alpha_{n+1}}}_{>2} \cdot t_{n}>2 t_{n}>t_{n}
$$

(pois, por hipótese, $\alpha_{n+1}<1$ e $\Delta \geq 2$ ).

Além disso, é relativamente fácil ver que $\varphi_{n+1}\left(0_{X}\right)=0_{E}$ (essa parte é imediata) e que, para todos $x, y \in X$,

$$
\begin{aligned}
\|x-y\|_{X} \leq t_{n} & \Rightarrow\left\|\frac{\alpha_{n+1}}{t_{n}} \cdot x-\frac{\alpha_{n+1}}{t_{n}} \cdot y\right\|_{X} \leq \alpha_{n+1} \\
& \Rightarrow\left\|\sigma\left(\frac{\alpha_{n+1}}{t_{n}} \cdot x\right)-\sigma\left(\frac{\alpha_{n+1}}{t_{n}} \cdot y\right)\right\|_{E}<\frac{M}{S_{n+1}+2^{n+1}} 2^{-(n+1)} \\
& \Rightarrow\left\|\varphi_{n+1}(x)-\varphi_{n+1}(y)\right\|_{E}<2^{-(n+1)}
\end{aligned}
$$

e

$$
\begin{aligned}
\|x-y\|_{X} \geq r_{n+1}=\frac{\Delta t_{n}}{\alpha_{n+1}} & \Rightarrow\left\|\frac{\alpha_{n+1}}{t_{n}} \cdot x-\frac{\alpha_{n+1}}{t_{n}} \cdot y\right\|_{X} \geq \Delta \\
& \Rightarrow\left\|\sigma\left(\frac{\alpha_{n+1}}{t_{n}} \cdot x\right)-\sigma\left(\frac{\alpha_{n+1}}{t_{n}} \cdot y\right)\right\|_{E} \geq M \\
& \Rightarrow\left\|\varphi_{n+1}(x)-\varphi_{n+1}(y)\right\|_{E} \geq S_{n+1}+2^{n+1}
\end{aligned}
$$

a partir do que concluímos que

$$
\begin{aligned}
\left\|\sum_{i=1}^{n+1}\left(\varphi_{i}(x)-\varphi_{i}(y)\right)\right\|_{E} & =\left\|\left(\varphi_{n+1}(x)-\varphi_{n+1}(y)\right)+\sum_{i=1}^{n}\left(\varphi_{i}(x)-\varphi_{i}(y)\right)\right\|_{E} \\
& \geq \underbrace{\left\|\varphi_{n+1}(x)-\varphi_{n+1}(y)\right\|_{E}}_{\geq S_{n+1}+2^{n+1}}-\underbrace{\left\|\sum_{i=1}^{n}\left(\varphi_{i}(x)-\varphi_{i}(y)\right)\right\|_{E}}_{\leq S_{n+1}} \\
& \geq\left(S_{n+1}+2^{n+1}\right)-S_{n+1}=2^{n+1}
\end{aligned}
$$

para todos $x, y \in X$ tais que $\|x-y\|_{X} \geq r_{n+1}$.

De modo análogo, dado $n \in \mathbb{N}$, podemos definir $\psi_{n+1}$ e $t_{n+1}>2 r_{n}$ a partir de $\psi_{1}, \ldots, \psi_{n}$ e de $0=t_{0}<r_{1}<t_{1}<\ldots<r_{n}$, bastando, para isso, substituir $\varphi$ por $\psi$ e $\sigma$ por $\omega$ na construção acima, e fazer as adaptações necessárias.

Por fim, precisamos, ainda, mostrar que $\lim _{n \rightarrow+\infty} r_{n}=\lim _{n \rightarrow+\infty} t_{n}=+\infty$. Para tanto, observe que, dado $n \in \mathbb{N}$, temos, por construção, que

$$
t_{n+1}>r_{n+1}>2 t_{n}>2^{2} r_{n}
$$

Por indução, disso concluímos que

$$
t_{n}>r_{n} \geq 2^{2(n-1)} r_{1}
$$


para todo $n \in \mathbb{N}$. E, uma vez que $\lim _{n \rightarrow+\infty} 2^{2(n-1)} r_{1}=+\infty$, segue do teorema do confronto que

$$
\lim _{n \rightarrow+\infty} r_{n}=\lim _{n \rightarrow+\infty} t_{n}=+\infty .
$$

Uma vez munidos desse resultado auxiliar, podemos, agora, provar o teorema 5.3.7.

Demonstração. Sejam $\left(r_{n}\right)_{n \in \mathbb{N}},\left(t_{n}\right)_{n \in \mathbb{N}_{0}},\left(\varphi_{n}\right)_{n \in \mathbb{N}}$ e $\left(\psi_{n}\right)_{n \in \mathbb{N}}$ tais como no lema 5.3.9. No que segue, utilizaremos tais sequências para construir a função $\Phi$.

Para começar, fixemos $x, y \in X$ de modo arbitrário e tomemos $n \in \mathbb{N}$ de modo que $\|x-y\|_{X} \leq t_{n-1}$. Como $\lim _{n \rightarrow+\infty} t_{n}=+\infty$, é imediato ver que um tal $n$ sempre existe. Além disso, uma vez que $0=t_{0}<r_{1}<t_{1}<r_{2}<t_{2}<\ldots$, também é fácil ver que, para todo $i \in \mathbb{N}$,

$$
i \geq n \Rightarrow r_{i}>t_{i-1} \geq t_{n-1} \Rightarrow\|x-y\|_{X} \leq t_{i-1}<r_{i}
$$

a partir do que segue de (5.14) e de (5.15) que

$$
\left\|\varphi_{i}(x)-\varphi_{i}(y)\right\|_{E} \leq 2^{-i} \text { e }\left\|\psi_{i}(x)-\psi_{i}(y)\right\|_{F} \leq 2^{-i}
$$

para todo $i \in \mathbb{N}$ tal que $i \geq n$. E, como $\sum_{i=1}^{+\infty} 2^{-i}$ converge, disso resulta, pelo Critério da comparação, que $\sum_{i=1}^{+\infty}\left\|\varphi_{i}(x)-\varphi_{i}(y)\right\|_{E}$ e $\sum_{i=1}^{+\infty}\left\|\psi_{i}(x)-\psi_{i}(y)\right\|_{F}$ convergem. Mas, mais do que isso, o critério nos diz também que

$$
\sum_{i=n}^{+\infty}\left\|\varphi_{i}(x)-\varphi_{i}(y)\right\|_{E} \leq \sum_{i=n}^{+\infty} 2^{-i}=\frac{2^{-n}}{1-\frac{1}{2}}=2^{-n+1}
$$

e que

$$
\sum_{i=n}^{+\infty}\left\|\psi_{i}(x)-\psi_{i}(y)\right\|_{F} \leq \sum_{i=n}^{+\infty} 2^{-i}=2^{-n+1} .
$$

Por outro lado, como $\left(E,\|\cdot\|_{E}\right)$ e $\left(F,\|\cdot\|_{F}\right)$ são espaços de Banach, segue da convergência das séries acima que $\sum_{i=1}^{+\infty}\left(\varphi_{i}(x)-\varphi_{i}(y)\right)$ e $\sum_{i=1}^{+\infty}\left(\psi_{i}(x)-\psi_{i}(y)\right)$ convergem em $\left(E,\|\cdot\|_{E}\right)$ e em $\left(F,\|\cdot\|_{F}\right)$, respectivamente. E, uma vez que $\|\cdot\|_{E}$ e $\|\cdot\|_{F}$ são contínuas, disso concluímos que

$$
\left\|\sum_{i=n}^{+\infty}\left(\varphi_{i}(x)-\varphi_{i}(y)\right)\right\|_{E} \leq \sum_{i=n}^{+\infty}\left\|\varphi_{i}(x)-\varphi_{i}(y)\right\|_{E} \leq 2^{-n+1} \leq 1
$$

e que

$$
\left\|\sum_{i=n}^{+\infty}\left(\psi_{i}(x)-\psi_{i}(y)\right)\right\|_{F} \leq \sum_{i=n}^{+\infty}\left\|\psi_{i}(x)-\psi_{i}(y)\right\|_{F} \leq 2^{-n+1} \leq 1 .
$$

Tais desigualdades nos serão muito úteis em breve. Mas, por hora, limitemo-nos a observar que, tomando $y=0_{X}$ e notando que $\varphi_{n}\left(0_{X}\right)=0_{E}$ e $\psi_{n}\left(0_{X}\right)=0_{F}$ para todo $n \in \mathbb{N}$ (por construção), podemos concluir que $\sum_{i=1}^{+\infty} \varphi_{i}(x)$ e $\sum_{i=1}^{+\infty} \psi_{i}(x)$ convergem, respectivamente, em $\left(E,\|\cdot\|_{E}\right)$ e em $\left(F,\|\cdot\|_{F}\right)$. E, como $x \in X$ é arbitrário, isso nos mostra que $\varphi: X \longrightarrow E$ e $\psi: X \longrightarrow F$ 
tais que $\varphi(x)=\sum_{i=1}^{+\infty} \varphi_{i}(x)$ e $\psi(x)=\sum_{i=1}^{+\infty} \psi_{i}(x)$ estão bem definidas.

A partir de $\varphi$ e de $\psi$, podemos definir a função $\varphi \oplus \psi: X \longrightarrow E \times F$ tal que $(\varphi \oplus \psi)(x)=$ $(\varphi(x), \psi(x))$. Nosso objetivo, agora, é mostrar que podemos tomar $\Phi=\varphi \oplus \psi$. Para isso, vamos, primeiramente, verificar que $\varphi$ e $\psi$ são uniformemente contínuas, a partir do que seguirá da proposição 5.1.3 que $\varphi \oplus \psi$ é uniformemente contínua e, portanto, também bornológica.

Seja $\varepsilon>0$ dado. Como $\lim _{n \rightarrow+\infty} 2^{-n+2}=0$, podemos fixar $n \in\{2,3, \ldots\}$ tal que $2^{-n+2}<\varepsilon$. Note que, sendo $\varphi_{i}$ uniformemente contínua para cada $i \in \mathbb{N}, \sum_{i=1}^{n-1} \varphi_{i}$ é, também, uniformemente contínua. Assim sendo, podemos tomar $\beta>0$ tal que

$$
\left\|\sum_{i=1}^{n-1}\left(\varphi_{i}(x)-\varphi_{i}(y)\right)\right\|_{E}<\frac{\varepsilon}{2}
$$

para todos $x, y \in X$ tais que $\|x-y\|_{X}<\beta$.

Por outro lado, segue de (5.16) que, para todos $x, y \in X$,

$$
\|x-y\|_{X} \leq t_{n-1} \Rightarrow\left\|\sum_{i=n}^{+\infty}\left(\varphi_{i}(x)-\varphi_{i}(y)\right)\right\|_{E} \leq 2^{-n+1}=\frac{2^{-n+2}}{2}<\frac{\varepsilon}{2} .
$$

Dessa forma, se tomarmos $\delta:=\min \left\{\beta, t_{n-1}\right\}$, teremos que

$$
\begin{aligned}
\|\varphi(x)-\varphi(y)\|_{E} & =\left\|\sum_{i=1}^{+\infty}\left(\varphi_{i}(x)-\varphi_{i}(y)\right)\right\|_{E} \\
& \leq\left\|\sum_{i=1}^{n-1}\left(\varphi_{i}(x)-\varphi_{i}(y)\right)\right\|_{E}+\left\|\sum_{i=n}^{+\infty}\left(\varphi_{i}(x)-\varphi_{i}(y)\right)\right\|_{E} \\
& <\frac{\varepsilon}{2}+\frac{\varepsilon}{2}=\varepsilon
\end{aligned}
$$

para todos $x, y \in X$ tais que $\|x-y\|_{X}<\delta$. E, como $\varepsilon>0$ é arbitrário, isso prova que $\varphi$ é uniformemente contínua. De modo análogo, pode-se mostrar que $\psi$ é uniformemente contínua, bastando, para isso, substituir $\varphi$ por $\psi, \varphi_{i}$ por $\psi_{i}, t_{n-1}$ por $r_{n},(5.16)$ por (5.17) e $E$ por $F$ na demonstração precedente.

Por fim, vamos mostrar que $\varphi \oplus \psi$ é expansível. Uma vez que $\varphi \oplus \psi$ é, também, bornológica, disso seguirá que $\varphi \oplus \psi$ é um mergulho grosseiro.

Dado $M>0$, devemos mostrar que existe $\Delta>0$ tal que

$$
\|(\varphi \oplus \psi)(x)-(\varphi \oplus \psi)(y)\|_{E \times F} \geq M
$$

quaisquer que sejam $x, y \in X$ tais que $\|x-y\|_{X} \geq \Delta$. Para tanto, comecemos fixando $m \in \mathbb{N}$ tal que $2^{m} \geq M+1$, e vamos provar que podemos tomar $\Delta=r_{m}$.

Sejam $x$ e $y$ dois pontos quaisquer de $X$ tais que $\|x-y\|_{X} \geq r_{m}$. Observe que o conjunto $J:=\left\{j \in \mathbb{N}: r_{j} \leq\|x-y\|_{X}\right\}$ é finito, uma vez que, por hipótese, $\lim _{j \rightarrow+\infty} r_{j}=+\infty$. Seja $n=\max (J)$. Por definição, disso resulta que $r_{n} \leq\|x-y\|_{X}<r_{n+1}$ e, portanto, temos duas possibilidades a considerar: ou $r_{n} \leq\|x-y\|_{X} \leq t_{n}$; ou $t_{n}<\|x-y\|_{X}<r_{n+1}$. No primeiro 
caso, segue de (5.12) e de (5.16) que

$$
\begin{aligned}
\|\varphi(x)-\varphi(y)\|_{E} & =\left\|\sum_{i=1}^{+\infty}\left(\varphi_{i}(x)-\varphi_{i}(y)\right)\right\|_{E} \\
& \geq \underbrace{\left\|\sum_{i=1}^{n}\left(\varphi_{i}(x)-\varphi_{i}(y)\right)\right\|_{E}}_{\geq 2^{n}}-\underbrace{\left\|\sum_{i=n+1}^{+\infty}\left(\varphi_{i}(x)-\varphi_{i}(y)\right)\right\|_{E}}_{\leq 1} \\
& \geq 2^{n}-1 .
\end{aligned}
$$

Já no segundo caso, temos, por (5.13) e (5.17), que

$$
\begin{aligned}
\|\psi(x)-\psi(y)\|_{F} & =\left\|\sum_{i=1}^{+\infty}\left(\psi_{i}(x)-\psi_{i}(y)\right)\right\|_{F} \\
& \geq \underbrace{\left\|\sum_{i=1}^{n}\left(\psi_{i}(x)-\psi_{i}(y)\right)\right\|_{F}}_{\geq^{n}}-\underbrace{\left\|\sum_{i=n+1}^{+\infty}\left(\psi_{i}(x)-\psi_{i}(y)\right)\right\|_{F}}_{\leq 1} \\
& \geq 2^{n}-1 .
\end{aligned}
$$

Assim sendo, em qualquer um dos casos, vale que

$\|(\varphi \oplus \psi)(x)-(\varphi \oplus \psi)(y)\|_{E \times F}=\|\varphi(x)-\varphi(y)\|_{E}+\|\psi(x)-\psi(y)\|_{F} \geq 2^{n}-1 \geq 2^{m}-1 \geq M$.

Por sua vez, como $x, y \in X$ tais que $\|x-y\|_{X} \geq r_{m}$ são arbitrários, isso mostra que, para todos $x, y \in X$,

$$
\|x-y\|_{X} \geq r_{m} \Rightarrow\|(\varphi \oplus \psi)(x)-(\varphi \oplus \psi)(y)\|_{E \times F} \geq M .
$$

E, sendo $M$ um real positivo qualquer, disso concluímos que $\varphi \oplus \psi$ é expansível.

O teorema 5.3.6 tem o seguinte corolário:

Corolário 5.3.10. Sejam $\left(X,\|\cdot\|_{X}\right)$ e $\left(E,\|\cdot\|_{E}\right)$ espaços de Banach, e $\sigma: X \longrightarrow B_{X}[0,1]$, $\varphi: X \longrightarrow E$ mergulhos uniformes. Nessas condições, existe um mergulho grosseiro de $\left(X,\|\cdot\|_{X}\right)$ em $\left(E \times E,\|\cdot\|_{E \times E}\right)$ o qual é, também, uniformemente contínuo.

Demonstração. Antes de mais nada, observe que, sendo a composição de mergulhos uniformes, $\varphi \circ \sigma: X \longrightarrow E$ é, ela própria, um mergulho uniforme (veja o corolário 3.4.12). Disso resulta, em particular, que $\varphi \circ \sigma$ é uniformemente contínua e não colapsada. Assim sendo, se mostrarmos que $\varphi \circ \sigma$ é, também, limitada, o resultado seguirá imediatamente do teorema 5.3.6.

Para isso, note, inicialmente, que $\varphi$ é bornológica (pois é uniformemente contínua). E, portanto, podemos fixar $M>0$ tal que $\|\varphi(x)-\varphi(y)\|_{E}<M$ para todos $x, y \in X$ tais que $\|x-y\|_{X} \leq 1$. Disso resulta, em particular, que

$$
\|\varphi(x)\|_{E}=\left\|\left(\varphi(x)-\varphi\left(0_{X}\right)\right)+\varphi\left(0_{X}\right)\right\|_{E} \leq\left\|\varphi(x)-\varphi\left(0_{X}\right)\right\|_{E}+\left\|\varphi\left(0_{X}\right)\right\|_{E}<M+\left\|\varphi\left(0_{X}\right)\right\|_{E}
$$

para todo $x \in B_{X}[0,1]$. E, uma vez que, por hipótese, $\sigma(X) \subseteq B_{X}[0,1]$, disso concluímos que

$$
(\varphi \circ \sigma)(X)=\varphi(\sigma(X)) \subseteq \varphi\left(B_{X}[0,1]\right) \subseteq B\left(0_{E}, R\right)
$$

em que $R:=M+\left\|\varphi\left(0_{X}\right)\right\|_{E}$. 
OBSERVAÇÃo 5.3.11. O espaço $\left(\ell_{2},\|\cdot\|_{2}\right)$ é um exemplo simples de espaço o qual pode ser uniformemente mergulhado em sua bola unitária e fechada. ${ }^{5}$ 


\section{Capítulo 6}

\section{ESTRUTURAS COARSE INVARIANTES À ESQUERDA}

Neste capítulo, vamos estudar, em mais detalhes, uma classe particular de estruturas coarse sobre grupos, nomeadamente, a das estruturas coarse invariantes à esquerda, a qual nada mais é do que a coleção das estruturas coarse para as quais existem bases formadas por conjuntos invariantes à esquerda. ${ }^{1}$ Os resultados aqui expostos foram, em grande parte, extraídos do manuscrito Coarse Geometry of Topological Groups [Ros17a], de Christian Rosendal, o qual foi, sem dúvidas, uma das principais fontes de inspiração para a realização deste trabalho.

Na primeira parte, exploraremos a relação entre o conjunto das estruturas coarse invariantes à esquerda em um grupo $(G, \cdot)$ e uma classe particular de ideais sobre $G$. O objetivo, aqui, é mostrar como podemos caracterizar uma estrutura coarse invariante à esquerda em $(G, \cdot)$ em termos de um certo ideal sobre $G$, e vice-versa.

Posteriormente, focaremos nossa atenção no caso particular da estrutura coarse à esquerda associada a um grupo topológico $(G, \cdot, \tau)$. Inicialmente, vamos introduzir os chamados conjuntos grosseiramente limitados e, utilizando os resultados da primeira parte, mostraremos como descrever a estrutura coarse à esquerda $\mathcal{E}_{L}$ em termos da coleção dos conjuntos grosseiramente limitados em $\left(G, \mathcal{E}_{L}\right)$. Em seguida, utilizaremos essa caracterização para provar que a estrutura coarse à esquerda associada ao grupo aditivo de um espaço normado coincide com a estrutura coarse limitada induzida pela norma.

\subsection{ESTRUTURAS COARSE INVARIANTES À ESQUERDA}

Nesta seção, vamos introduzir e caracterizar as chamadas estruturas coarse invariantes à esquerda em um grupo $(G, \cdot)$. Antes disso, porém, recordemos que, dado um grupo $(G, \cdot)$, dizemos que um subconjunto $E \subseteq G \times G$ é invariante à esquerda em $(G, \cdot)$ se, para todo $(x, y) \in G \times G$ e todo $\lambda \in G$, valer que

$$
(x, y) \in E \Rightarrow(\lambda x, \lambda y) \in E .
$$

\footnotetext{
${ }^{1}$ Observe a enorme similaridade entre esta definição e a de uniformidade invariante à esquerda (no apêndice A), a qual é, na verdade, mais uma consequência da dualidade entre as noções de uniformidade e de estrutura coarse e dos conceitos auxiliares que as permeiam (como, por exemplo, o de base). Em razão dessa semelhança, muitos dos resultados referentes às uniformidades invariantes à esquerda poderão ser, prontamente, adaptados à classe das estruturas coarse invariantes à esquerda (e vice-versa).
} 
Definição 6.1.1 (Estrutura COARSe invariante À esquerda). Dado um grupo $(G, \cdot)$, dizemos que uma estrutura coarse $\mathcal{E}$ sobre $G$ é invariante à esquerda em $(G, \cdot)$ se $\mathcal{E}$ possuir uma base formada por conjuntos invariantes à esquerda em $(G, \cdot)$.

Vejamos, a seguir, alguns exemplos de estruturas coarse invariantes à esquerda.

Exemplos 6.1.2. Seja $(G, \cdot)$ um grupo.

a) As estruturas coarse $\mathcal{E}_{\max }:=\mathcal{P}(G \times G)$ e $\mathcal{E}_{\text {min }}:=\mathcal{P}(\Delta(G))$ são ambas invariantes à esquerda em $(G, \cdot)$, pois possuem bases, $\mathscr{B}_{\max }:=\{G \times G\}$ e $\mathscr{B}_{\min }:=\{\Delta(G)\}$, respectivamente, formadas por subconjuntos invariantes à esquerda.

b) A estrutura coarse limitada associada a uma pseudométrica invariante à esquerda $d$ sobre $G$ é invariante à esquerda em $(G, \cdot)$, pois tem como base a coleção $\mathscr{B}_{d}:=\left\{E_{\alpha}^{d}: \alpha>0\right\}$, a qual é formada por subconjuntos invariantes à esquerda. Com efeito, sendo $d$ invariante à esquerda, temos que $d(x, y)=d(\lambda x, \lambda y)$ quaisquer que sejam $(x, y) \in G \times G$ e $\lambda \in G$. E, com isso em mente, é imediato ver que, para todos $x, y, \lambda \in G$ e todo $\alpha>0$,

$$
(x, y) \in E_{\alpha}^{d} \Rightarrow d(x, y)<\alpha \Rightarrow d(\lambda x, \lambda y)<\alpha \Rightarrow(\lambda x, \lambda y) \in E_{\alpha}^{d}
$$

c) Sejam $(H, \cdot)$ um subgrupo de $(G, \cdot), \mathcal{E}_{G}$ uma estrutura coarse sobre $G$ e

$$
\mathcal{E}_{H}:=\left\{E \cap(H \times H): E \in \mathcal{E}_{G}\right\}
$$

a estrutura coarse induzida por $\mathcal{E}_{G}$ sobre $H$ (veja o exemplo 4.1.10-c)). No que segue, mostraremos que, se $\mathcal{E}_{G}$ for invariante à esquerda em $(G, \cdot)$, então $\mathcal{E}_{H}$ será invariante à esquerda em $(H, \cdot)$.

Para isso, fixemos, inicialmente, uma base $\mathscr{B}_{G}$ de $\mathcal{E}_{G}$ formada por conjuntos invariantes à esquerda em $(G, \cdot){ }^{2}$ Observe que, sendo $\mathscr{B}_{G}$ uma base de $\mathcal{E}_{G}$, segue do exemplo 4.1.10-c) que

$$
\mathscr{B}_{H}:=\left\{B \cap(H \times H): B \in \mathscr{B}_{G}\right\}
$$

é base de $\mathcal{E}_{H}$. Além disso, tendo em vista que $H \subseteq G$ e que todo $B \in \mathscr{B}_{G}$ é invariante à esquerda em $(G, \cdot)$, é imediato ver que $(\lambda x, \lambda y) \in B$ quaisquer que sejam $B \in \mathscr{B}_{G},(x, y) \in B$ e $\lambda \in H$. E, sendo $(H, \cdot)$ um subgrupo de $(G, \cdot)$ (e, portanto, fechado em relação à operação de grupo), disso concluímos que, para todo $B \in \mathscr{B}_{G}$ e todos $x, y, \lambda \in H$,

$$
\begin{aligned}
(x, y) \in B \cap(H \times H) & \Rightarrow[(x, y) \in B \text { e } x, y \in H] \Rightarrow(\lambda x, \lambda y) \in B \text { e } \lambda x, \lambda y \in H] \\
& \Rightarrow(\lambda x, \lambda y) \in B \cap(H \times H),
\end{aligned}
$$

do que segue que $\mathscr{B}_{H}$ é uma base de $\mathcal{E}_{H}$ formada por subconjuntos invariantes à esquerda.

A seguir, vamos caracterizar as estruturas coarse invariantes à esquerda em termos de seus conjuntos geradores.

Proposição 6.1.3. Sejam $(G, \cdot)$ um grupo e $\mathcal{E}$ uma estrutura coarse sobre $G$. Nessas condições, $\mathcal{E}$ será invariante à esquerda em $(G, \cdot)$ se, e somente se, existir uma coleção $\mathcal{S} \subseteq \mathcal{P}(G \times G)$ de conjuntos invariantes à esquerda em $(G, \cdot)$ tal que $\mathcal{E}=\mathcal{E}_{\mathcal{S}}$.

\footnotetext{
${ }^{2}$ Uma tal base certamente existe, uma vez que, por hipótese, $\mathcal{E}_{G}$ é invariante à esquerda.
} 
Demonstração. $(\Rightarrow)$ Seja $\mathcal{S} \subseteq \mathcal{P}(G \times G)$ uma coleção de subconjuntos invariantes à esquerda tal que $\mathcal{E}=\mathcal{E}_{\mathcal{S}}$. Observe que, pela proposição 4.2.7, temos que

$$
\begin{aligned}
\mathscr{B}_{\mathcal{S}}:=\{ & F_{1} \circ \ldots \circ F_{n} \subseteq G \times G: n \in \mathbb{N} \text { e, para cada } i \in\{1, \ldots, n\}, \\
& \left.F_{i} \text { é uma reunião finita de elementos de }\{\Delta\} \cup \mathcal{S} \cup \mathcal{S}^{-1}\right\}
\end{aligned}
$$

é uma base de $\mathcal{E}$.

Além disso, é fácil ver que, sendo o resultado da composição e da união de conjuntos invariantes à esquerda, um elemento qualquer de $\mathscr{B}_{\mathcal{S}}$ é, ele próprio, invariante à esquerda (veja a proposição 2.8.6). E, assim sendo, $\mathscr{B}_{\mathcal{S}}$ é uma base de $\mathcal{E}$ formada por conjuntos invariantes à esquerda.

$(\Leftarrow)$ Suponhamos, agora, que $\mathcal{E}$ seja invariante à esquerda. Nesse caso, existe uma base $\mathscr{B}$ de $\mathcal{E}$ a qual é formada por subconjuntos invariantes à esquerda. E, sendo $\mathscr{B}$ uma base de $\mathcal{E}$, temos, em particular, que $\mathcal{E}_{\mathscr{B}}=\mathcal{E}$, a partir do que concluímos, imediatamente, o resultado.

Seja $(G, \cdot)$ um grupo. No capítulo 4 , vimos que a intersecção $\mathcal{E}$ de uma família não vazia $\left(\mathcal{E}_{i}\right)_{i \in I}$ de estruturas coarse sobre $G$ é, também, uma estrutura coarse sobre $G$ (proposição 4.2.1). Tendo isso em vista, é razoável perguntar o que podemos inferir sobre $\mathcal{E}$ no caso em que $\mathcal{E}_{i}$ é invariante à esquerda em $(G, \cdot)$ para todo $i \in I$. A proposição a seguir responde a essa questão.

Proposição 6.1.4. Sejam $(G, \cdot)$ um grupo e $\left(\mathcal{E}_{i}\right)_{i \in I}$ uma família não vazia de estruturas coarse sobre $G$ invariantes à esquerda em $(G, \cdot)$. Nessas condições, $\mathcal{E}:=\bigcap_{i \in I} \mathcal{E}_{i}$ é, também, invariante à esquerda.

Demonstração. Para cada $i \in I$, seja $\mathscr{B}_{i}$ uma base de $\mathcal{E}_{i}$ formada por subconjuntos invariantes à esquerda. Pela proposição 4.2 .3 , disso segue que

$$
\mathscr{B}:=\left\{B \subseteq G \times G: \exists\left(B_{i}\right)_{i \in I} \text { t.q. } B_{i} \in \mathscr{B}_{i} \text { para todo } i \in I \text { e } B=\bigcap_{i \in I} B_{i}\right\}
$$

é uma base de $\mathcal{E}$. Assim sendo, se mostrarmos que cada $B \in \mathscr{B}$ é invariante à esquerda, disso resultará, por conseguinte, que $\mathcal{E}$ é invariante à esquerda.

Seja $B \in \mathscr{B}$ um conjunto arbitrário. Por definição, disso segue que existe $\left(B_{i}\right)_{i \in I}$ tal que $B_{i} \in \mathscr{B}_{i}$ para todo $i \in I$ e $B=\bigcap_{i \in I} B_{i}$. Observe que, para cada $i \in I, B_{i}$ é invariante à esquerda (pois $B_{i} \in \mathscr{B}_{i}$ e, por hipótese, $\mathscr{B}_{i}$ é formada por subconjuntos invariantes à esquerda). E, sendo $B$ a intersecção de conjuntos invariantes à esquerda, segue da proposição 2.8.6 que ele é, também, invariante à esquerda.

ExEMPLo 6.1.5. A estrutura coarse à esquerda $\mathcal{E}_{L}$ associada a um grupo topológico $(G, \cdot, \tau)$ é invariante à esquerda em $(G, \cdot)$, uma vez que, por definição,

$$
\mathcal{E}_{L}=\bigcap_{d \in \mathcal{D}} \mathcal{E}_{d}
$$

e cada $d \in \mathcal{D}$ é uma pseudométrica sobre $G$ invariante à esquerda em $(G, \cdot)$.

O nosso objetivo, a seguir, é provar que, dado um grupo $(G, \cdot)$, podemos estabelecer uma estreita conexão entre o conjunto das estruturas coarse sobre $G$ invariantes à esquerda em $(G, \cdot)$ e uma classe especial $\mathcal{I}$ de ideais sobre $G$. Mais especificamente, mostraremos como descrever uma estrutura coarse invariante à esquerda em $(G, \cdot)$ em termos de um ideal em $\mathcal{I}$, e vice-versa. Para isso, precisaremos, em primeiro lugar, estabelecer uma notação conveniente, bem como fazer algumas definições e considerações preliminares, as quais serão úteis mais adiante. 
DefiniÇÃo 6.1.6. Sejam $(G, \cdot)$ um grupo e $e \in G$ o elemento neutro de $(G, \cdot)$. Dado um conjunto $E \subseteq G \times G$ invariante à esquerda em $(G, \cdot)$, denotaremos por $A_{E}$ o conjunto

$$
\{x \in G:(e, x) \in E\} .
$$

Reciprocamente, dado um subconjunto $A \subseteq G$, denotaremos por $E_{A}$ o conjunto

$$
\left\{(x, y) \in G \times G: x^{-1} y \in A\right\} .
$$

Com o auxílio da notação introduzida pela definição 6.1.6, podemos, rapidamente, chegar a algumas conclusões interessantes. Inicialmente, observe que, se $E \subseteq G \times G$ é um conjunto invariante à esquerda, então, para todo $(x, y) \in G \times G$, vale que

$$
(x, y) \in E \Leftrightarrow\left(e, x^{-1} y\right) \in E \Leftrightarrow x^{-1} y \in A_{E},
$$

a partir do que concluímos que $E=\left\{(x, y) \in G \times G: x^{-1} y \in A_{E}\right\}$. Analogamente, dado $A \subseteq G$, é imediato ver que $E_{A}$ é sempre invariante à esquerda e que, para todo $x \in G$,

$$
x \in A \Leftrightarrow e^{-1} x \in A \Leftrightarrow(e, x) \in E_{A},
$$

do que segue que $A=\left\{x \in G:(e, x) \in E_{A}\right\}$. Em particular, disso resulta que

$$
A_{E_{A}}=\left\{x \in G:(e, x) \in E_{A}\right\}=A
$$

$\mathrm{e}$

$$
E_{A_{E}}=\left\{(x, y) \in G \times G: x^{-1} y \in A_{E}\right\}=E
$$

para todo $A \subseteq G$ e todo $E \subseteq G \times G$ tal que $E$ é invariante à esquerda. Com isso, mostramos o seguinte resultado:

Proposição 6.1.7. Sejam $(G, \cdot)$ um grupo e

$$
L:=\{E \subseteq G \times G: \text { E é invariante à esquerda em }(G, \cdot)\} .
$$

Nessas condições, a função

$$
\begin{aligned}
\varphi: \mathcal{P}(G) & \longrightarrow L \\
A & \longmapsto E_{A}
\end{aligned}
$$

é uma bijeção, e $\varphi^{-1}: L \longrightarrow \mathcal{P}(G)$ é tal que $\varphi^{-1}(E)=A_{E}$.

A seguir, vamos enunciar algumas consequências simples da definição 6.1.6.

Proposição 6.1.8. Sejam $(G, \cdot)$ um grupo e e $\in G$ o elemento neutro de $(G, \cdot)$. Para todos $A, B \subseteq G$, temos que

a) $E_{A}^{-1}=E_{A^{-1}}$;

b) $E_{A} \cup E_{B}=E_{A \cup B}$;

c) $E_{A} \cap E_{B}=E_{A \cap B}$;

d) $E_{A} \circ E_{B}=E_{B A}$;

e) $E_{A}[B]=B A$; 
f) $A \subseteq B$ se, e somente se, $E_{A} \subseteq E_{B}$.

Demonstração. Dados $A, B \subseteq G$, temos que

$$
\begin{aligned}
& \left.E_{A^{-1}=\{(x, y) \in G \times G}:(y, x) \in E_{A}\right\}=\left\{(x, y) \in G \times G: y^{-1} x \in A\right\} \\
& =\left\{(x, y) \in G \times G:\left(x^{-1} y\right)^{-1} \in A\right\}=\left\{(x, y) \in G \times G: x^{-1} y \in A^{-1}\right\}=E_{A^{-1}}, \\
& E_{A} \cup E_{B}=\left\{(x, y) \in G \times G:(x, y) \in E_{A} \text { ou }(x, y) \in E_{B}\right\} \\
& =\left\{(x, y) \in G \times G: x^{-1} y \in A \text { ou } x^{-1} y \in B\right\} \\
& =\left\{(x, y) \in G \times G: x^{-1} y \in A \cup B\right\}=E_{A \cup B}, \\
& \qquad \begin{aligned}
E_{A} \cap E_{B} & =\left\{(x, y) \in G \times G:(x, y) \in E_{A} \text { e }(x, y) \in E_{B}\right\} \\
& =\left\{(x, y) \in G \times G: x^{-1} y \in A \text { e } x^{-1} y \in B\right\} \\
& =\left\{(x, y) \in G \times G: x^{-1} y \in A \cap B\right\}=E_{A \cap B},
\end{aligned}
\end{aligned}
$$

e

$$
\begin{aligned}
E_{A}[B] & =\left\{y \in G: \exists b \in B \text { t.q. }(b, y) \in E_{A}\right\}=\left\{y \in G: \exists b \in B \text { t.q. } b^{-1} y \in A\right\} \\
& =\{y \in G: \exists b \in B \text { e } \exists a \in A \text { t.q. } y=b a\}=B A .
\end{aligned}
$$

Isso mostra a), b), c) e e). Vamos, agora, provar o item d). Dado $(x, y) \in G \times G$, temos que

$$
(x, y) \in E_{A} \circ E_{B} \Rightarrow \exists z \in G \text { t.q. }(x, z) \in E_{B} \text { e }(z, y) \in E_{A} .
$$

E, para todo $z \in G$,

$$
(x, z) \in E_{B} \text { e }(z, y) \in E_{A} \Rightarrow x^{-1} z \in B \text { e } z^{-1} y \in A \Rightarrow x^{-1} y \in B A \Rightarrow(x, y) \in E_{B A} .
$$

Dessa forma,

$$
(x, y) \in E_{A} \circ E_{B} \Rightarrow(x, y) \in E_{B A} .
$$

Reciprocamente, dado $(x, y) \in E_{B A}$, temos que $x^{-1} y \in B A$ e, por definição, disso segue que existem $a \in A$ e $b \in B$ tais que $x^{-1} y=b a$. Observe que

$$
x^{-1} y=b a \Rightarrow x b=y a^{-1} .
$$

Assim sendo, se tomarmos $z:=x b=y a^{-1}$, teremos que

$$
x^{-1} z=x^{-1}(x b)=b \in B
$$

e

$$
z^{-1} y=\left(y a^{-1}\right)^{-1} y=\left(a y^{-1}\right) y=a \in A .
$$

Disso resulta que $(x, z) \in E_{B}$ e $(z, y) \in E_{A}$, a partir do que concluímos que $(x, y) \in E_{A} \circ E_{B}$, completando, assim, a demonstração de que $E_{A} \circ E_{B}=E_{B A}$.

Por fim, devemos mostrar que também vale f). Para isso, suponhamos, inicialmente, que 
$A \subseteq B$. Nesse caso, segue imediatamente da definição 6.1 .6 que

$$
E_{A}:=\left\{(x, y) \in G \times G: x^{-1} y \in A\right\} \subseteq\left\{(x, y) \in G \times G: x^{-1} y \in B\right\}=E_{B} .
$$

Da mesma forma, é fácil ver que, se $E_{A} \subseteq E_{B}$, então

$$
A=\left\{x \in G:(e, x) \in E_{A}\right\} \subseteq\left\{x \in G:(e, x) \in E_{B}\right\}=B .
$$

Estamos, agora, quase prontos para provar o principal teorema desta seção. Antes disso, porém, faremos uma última definição.

DefiniÇão 6.1.9. Seja $(G, \cdot)$ um grupo. Dada uma estrutura coarse $\mathcal{E}$ sobre $G$, denotaremos por $\mathcal{A}_{\mathcal{E}}$ o conjunto

$$
\left\{A \subseteq G: E_{A} \in \mathcal{E}\right\}
$$

Reciprocamente, dada uma coleção $\mathcal{A} \subseteq \mathcal{P}(G)$, denotaremos por $\mathcal{E}_{\mathcal{A}}$ o conjunto

$$
\left\{E \subseteq G \times G: \exists A \in \mathcal{A} \text { t.q. } E \subseteq E_{A}\right\} .
$$

OBSERVAÇÃo 6.1.10. Dados um grupo $(G, \cdot)$ e uma estrutura coarse $\mathcal{E}$ sobre $G$, não é difícil provar que

$$
\mathcal{A}_{\mathcal{E}}=\left\{A \subseteq G: \exists E \in \mathcal{E} \text { invariante à esquerda em }(G, \cdot) \text { t.q. } A \subseteq A_{E}\right\} .
$$

Observe que, de certa forma, isso implica uma simetria entre as definições de $\mathcal{E}_{\mathcal{A}}$ e de $\mathcal{A}_{\mathcal{E}}$.

Uma vez munidos da terminologia introduzida em 6.1.9, podemos, sem mais delongas, enunciar o nosso grande resultado.

TeOrema 6.1.11. Sejam $(G, \cdot)$ um grupo, $e \in G$ o elemento neutro de $(G, \cdot), \mathscr{C}$ a coleção das estruturas coarse sobre $G$ invariantes à esquerda em $(G, \cdot)$, e $\mathcal{I}$ a coleção dos ideais $\mathcal{A}$ sobre $G$ tais que $\{e\} \in \mathcal{A}, A^{-1} \in \mathcal{A}$ para todo $A \in \mathcal{A}$ e $A B \in \mathcal{A}$ quaisquer que sejam $A, B \in \mathcal{A}$. Nessas condições:

a) $\mathcal{E}_{\mathcal{A}} \in \mathscr{C}$ para todo $\mathcal{A} \in \mathcal{I}$;

b) $\mathcal{A}_{\mathcal{E}} \in \mathcal{I}$ para todo $\mathcal{E} \in \mathscr{C}$;

c) A função $\Phi: \mathcal{I} \longrightarrow \mathscr{C}$ tal que $\Phi(\mathcal{A})=\mathcal{E}_{\mathcal{A}}$ é uma bijeção, e $\Phi^{-1}: \mathscr{C} \longrightarrow \mathcal{I}$ é tal que $\Phi^{-1}(\mathcal{E})=\mathcal{A}_{\mathcal{E}}$

Demonstração. a) Dado $\mathcal{A} \in \mathcal{I}$, definamos $\mathscr{B}_{\mathcal{A}}:=\left\{E_{A}: A \in \mathcal{A}\right\}$. Vamos mostrar que $\mathscr{B}_{\mathcal{A}}$ é base de uma estrutura coarse sobre $G$. Para isso, precisamos provar que $\mathscr{B}_{\mathcal{A}}$ satisfaz as condições de a) a d) da proposição 4.1.8.

Inicialmente, observe que

$$
\left(\{e\} \in \mathcal{A} \text { e } E_{\{e\}}:=\left\{(x, y) \in G \times G: x^{-1} y=e\right\}=\Delta\right) \Rightarrow \Delta \in \mathscr{B}_{\mathcal{A}} .
$$

Além disso, para todos $A, B \subseteq G$, temos que

$$
\begin{gathered}
A, B \in \mathcal{A} \Rightarrow A \cup B \in \mathcal{A} \Rightarrow E_{A \cup B} \in \mathscr{B}_{\mathcal{A}} \Rightarrow E_{A} \cup E_{B} \in \mathscr{B}_{\mathcal{A}} \\
A \in \mathcal{A} \Rightarrow A^{-1} \in \mathcal{A} \Rightarrow E_{A^{-1}} \in \mathscr{B}_{\mathcal{A}} \Rightarrow E_{A}^{-1} \in \mathscr{B}_{\mathcal{A}}
\end{gathered}
$$


e

$$
A, B \in \mathcal{A} \Rightarrow B A \in \mathcal{A} \Rightarrow E_{B A} \in \mathscr{B}_{\mathcal{A}} \Rightarrow E_{A} \circ E_{B} \in \mathscr{B}_{\mathcal{A}},
$$

o que, por sua vez, completa a demonstração. Observe ainda que, pela observação 4.1.6, fica claro que a estrutura coarse gerada por $\mathscr{B}_{\mathcal{A}}$ não deve ser outra senão $\mathcal{E}_{\mathcal{A}}$. E, uma vez que $E_{A}$ é invariante à esquerda em $(G, \cdot)$ para todo $A \in \mathcal{A}$, disso concluímos, por fim, que $\mathcal{E}_{\mathcal{A}} \in \mathscr{C}_{\text {. }}$.

b) Dado $\mathcal{E} \in \mathscr{C}$, vamos mostrar que $\mathcal{A}_{\mathcal{E}}$ é um ideal sobre $G$ tal que $\{e\} \in \mathcal{A}_{\mathcal{E}}, A^{-1} \in \mathcal{A}_{\mathcal{E}}$ para todo $A \in \mathcal{A}_{\mathcal{E}}$ e $A B \in \mathcal{A}_{\mathcal{E}}$ quaisquer que sejam $A, B \in \mathcal{A}_{\mathcal{E}}$. Para isso, notemos, inicialmente, que

$$
\left(\Delta \in \mathcal{E} \text { e } E_{\{e\}}=\Delta\right) \Rightarrow\{e\} \in \mathcal{A}_{\mathcal{E}}
$$

Além disso, dados $A, B \subseteq G$, temos que

$$
\begin{gathered}
A, B \in \mathcal{A}_{\mathcal{E}} \Rightarrow E_{A}, E_{B} \in \mathcal{E} \Rightarrow E_{A} \cup E_{B} \in \mathcal{E} \Rightarrow E_{A \cup B} \in \mathcal{E} \Rightarrow A \cup B \in \mathcal{A}_{\mathcal{E}} \\
\left(A \in \mathcal{A}_{\mathcal{E}} \text { e } B \subseteq A\right) \Rightarrow\left(E_{A} \in \mathcal{E} \text { e } E_{B} \subseteq E_{A}\right) \Rightarrow E_{B} \in \mathcal{E} \Rightarrow B \in \mathcal{A}_{\mathcal{E}} \\
A \in \mathcal{A}_{\mathcal{E}} \Rightarrow E_{A} \in \mathcal{E} \Rightarrow E_{A}^{-1} \in \mathcal{E} \Rightarrow E_{A^{-1}} \in \mathcal{E} \Rightarrow A^{-1} \in \mathcal{A}_{\mathcal{E}}
\end{gathered}
$$

e

$$
A, B \in \mathcal{A}_{\mathcal{E}} \Rightarrow E_{A}, E_{B} \in \mathcal{E} \Rightarrow E_{B} \circ E_{A} \in \mathcal{E} \Rightarrow E_{A B} \in \mathcal{E} \Rightarrow A B \in \mathcal{A}_{\mathcal{E}}
$$

E, como $A, B \subseteq G$ são arbitrários, isso completa a prova de que $\mathcal{A}_{\mathcal{E}} \in \mathcal{I}$.

c) Seja $\mathcal{E} \in \mathscr{C}$ dado. Note que, para todo $E \subseteq G \times G$,

$$
E \in \mathcal{E}_{\left(\mathcal{A}_{\mathcal{E}}\right)} \Rightarrow \exists A \in \mathcal{A}_{\mathcal{E}} \text { t.q. } E \subseteq E_{A} \Rightarrow \exists A \subseteq G \text { t.q. }\left(E_{A} \in \mathcal{E} \text { e } E \subseteq E_{A}\right) \Rightarrow E \in \mathcal{E} .
$$

Reciprocamente, dado $E \in \mathcal{E}$, podemos fixar $F \in \mathcal{E}$ invariante à esquerda em $(G, \cdot)$ tal que $E \subseteq F$ (pois, por hipótese, $\mathcal{E}$ é invariante à esquerda). Observe que

$$
\left(F \in \mathcal{E} \text { e } E_{A_{F}}=F\right) \Rightarrow A_{F} \in \mathcal{A}_{\mathcal{E}}
$$

E, por sua vez,

$$
\left(A_{F} \in \mathcal{A}_{\mathcal{E}} \text { e } E \subseteq F=E_{A_{F}}\right) \Rightarrow E \in \mathcal{E}_{\left(\mathcal{A}_{\mathcal{E}}\right)} .
$$

Isso mostra que $E \in \mathcal{E}_{\left(\mathcal{A}_{\mathcal{E}}\right)}$. E, sendo $E \in \mathcal{E}$ arbitrário, disso resulta que $\mathcal{E}_{\left(\mathcal{A}_{\mathcal{E}}\right)}=\mathcal{E}$.

Analogamente, dado $\mathcal{A} \in \mathcal{I}$, temos que, para todo $A \subseteq G$,

$$
A \in \mathcal{A}_{\left(\mathcal{E}_{\mathcal{A}}\right)} \Leftrightarrow E_{A} \in \mathcal{E}_{\mathcal{A}} \Leftrightarrow \exists B \in \mathcal{A} \text { t.q. } E_{A} \subseteq E_{B} \Leftrightarrow \exists B \in \mathcal{A} \text { t.q. } A \subseteq B \Leftrightarrow A \in \mathcal{A} \text {, }
$$

do que segue que $\mathcal{A}=\mathcal{A}_{\left(\mathcal{E}_{\mathcal{A}}\right)}$.

Assim sendo, se definirmos $\Psi: \mathscr{C} \longrightarrow \mathcal{I}$ tal que $\Psi(\mathcal{E})=\mathcal{A}_{\mathcal{E}}$, teremos que

$$
(\Psi \circ \Phi)(\mathcal{A})=\Psi(\Phi(\mathcal{A}))=\Psi\left(\mathcal{E}_{\mathcal{A}}\right)=\mathcal{A}_{\left(\mathcal{E}_{\mathcal{A}}\right)}=\mathcal{A}
$$

$\mathrm{e}$

$$
(\Phi \circ \Psi)(\mathcal{E})=\Phi(\Psi(\mathcal{E}))=\Phi\left(\mathcal{A}_{\mathcal{E}}\right)=\mathcal{E}_{\left(\mathcal{A}_{\mathcal{E}}\right)}=\mathcal{E}
$$

quaisquer que sejam $\mathcal{A} \in \mathcal{I}$ e $\mathcal{E} \in \mathscr{C}$, a partir do que concluímos que $\Phi$ e $\Psi$ são inversas uma da outra. 


\subsection{Conjuntos GRosseiramente limitados}

A toda estrutura coarse $\mathcal{E}$ sobre um conjunto $X$, podemos associar, naturalmente, a noção de conjunto grosseiramente limitado.

Definição 6.2.1. Dada uma estrutura coarse $\mathcal{E}$ sobre um conjunto $X$, dizemos que um conjunto $A \subseteq X$ é grosseiramente limitado em $(X, \mathcal{E})$ se $A \times A \in \mathcal{E}$.

NotaÇÃo: Dado um espaço coarse $(X, \mathcal{E})$, denotaremos por $\mathcal{C B}_{\mathcal{E}}{ }^{3}$ a coleção dos conjuntos grosseiramente limitados em $(X, \mathcal{E})$.

ObservaÇÃo 6.2.2. Sejam $(X, \mathcal{E})$ um espaço coarse e $A, B \subseteq X$ subconjuntos quaisquer. Observe que

$$
\left(A \in \mathcal{C B}_{\mathcal{E}} \text { e } B \subseteq A\right) \Rightarrow(A \times A \in \mathcal{E} \text { e } B \times B \subseteq A \times A) \Rightarrow B \times B \in \mathcal{E} \Rightarrow B \in \mathcal{C B}_{\mathcal{E}}
$$

Disso concluímos, em particular, que $\emptyset \in \mathcal{C B}_{\mathcal{E}}$ qualquer que seja a estrutura coarse $\mathcal{E}$ sobre $X$.

Antes de prosseguirmos, vamos determinar $\mathcal{C B}_{\mathcal{E}}$ em alguns casos simples.

Exemplos 6.2.3. Seja $X$ um conjunto arbitrário.

a) Qualquer subconjunto de $X$ é grosseiramente limitado em $(X, \mathcal{P}(X \times X))$. Assim sendo,

$$
\mathcal{C B}_{\mathcal{P}(X \times X)}=\mathcal{P}(X)
$$

b) Seja $\mathcal{E}_{\min }:=\mathcal{P}(\Delta(X))$. Observe que, para todo $x \in X$,

$$
\left(\{(x, x)\} \subseteq \Delta(X) \text { e } \Delta(X) \in \mathcal{E}_{\min }\right) \Rightarrow\{(x, x)\} \in \mathcal{E}_{\min } \Rightarrow\{x\} \in \mathcal{C B}_{\mathcal{E}_{\min }}
$$

Por outro lado, dado $A \subseteq X$ com mais de um elemento, é fácil ver que, para todos $x, y \in A$ tais que $x \neq y$, vale que $\{(x, y)\} \subseteq A \times A$ e que $\{(x, y)\} \notin \mathcal{E}_{\text {min }}$, a partir do que concluímos que $A \times A \notin \mathcal{E}_{\text {min }}$. Sendo assim, devemos ter, necessariamente,

$$
\mathcal{C B}_{\mathcal{E}_{\min }}=\{\emptyset\} \cup\{\{x\}: x \in X\} .
$$

c) Sejam $Y \subseteq X$ um conjunto não vazio, $\mathcal{E}_{X}$ uma estrutura coarse sobre $X$ e

$$
\mathcal{E}_{Y}:=\left\{E \subseteq Y \times Y: E \in \mathcal{E}_{X}\right\}
$$

a estrutura coarse induzida por $\mathcal{E}_{X}$ sobre $Y$. Note que, da definição de $\mathcal{E}_{Y}$, resulta que

$$
\mathcal{C B}_{\mathcal{E}_{Y}}:=\left\{A \subseteq Y: A \times A \in \mathcal{E}_{Y}\right\}=\left\{A \subseteq Y: A \times A \in \mathcal{E}_{X}\right\}=\mathcal{C B}_{\mathcal{E}_{X}} \cap \mathcal{P}(Y)
$$

d) Sejam $d$ uma pseudométrica sobre $X$ e

$$
\mathcal{E}_{d}:=\{E \subseteq X \times X:\{d(x, y):(x, y) \in E\} \text { é limitado }\}
$$

a estrutura coarse limitada induzida por $d$ sobre $X$. Observe que, para todo $A \subseteq X$,

$$
A \times A \in \mathcal{E}_{d} \Leftrightarrow\{d(x, y): x, y \in A\} \text { é limitado } \Leftrightarrow \operatorname{diam}_{d}(A) \text { é finito. }
$$

\footnotetext{
${ }^{3} \mathrm{~A}$ notação $\mathcal{C B}_{\mathcal{E}}$ remete ao termo "coarsely bounded sets", o qual nada mais é do que o equivalente em inglês para "conjuntos grosseiramente limitados".
} 
Disso segue que $\mathcal{C B}_{\mathcal{E}_{d}}$ é, simplesmente, a coleção dos subconjuntos de $X$ de diâmetro finito em relação a $d$.

Observe, em particular, que $X \times X \in \mathcal{E}_{d}$ se, e somente se, $\operatorname{diam}_{d}(X)$ é finito. Tendo em vista o exemplo 4.4.33-b), disso segue que $\operatorname{diam}_{d}(X)$ é finito se, e somente se, $\left(X, \mathcal{E}_{d}\right)$ é grosseiramente equivalente a um ponto. E isso, por sua vez, torna rigorosa a ideia intuitiva de que conjuntos limitados têm "tamanho insignificante" quando o que está em jogo é a geometria de larga escala.

e) Seja

$$
\mathcal{E}:=\{E \subseteq X \times X: E \backslash \Delta \text { é finito }\}
$$

a estrutura coarse discreta sobre $X$. Vamos mostrar que

$$
\mathcal{C B}_{\mathcal{E}}=\{A \subseteq X: A \text { é finito }\}
$$

Com efeito, dado $A \subseteq X$ finito, temos que $A \times A$ é finito e, em particular, $(A \times A) \backslash \Delta$ é finito, do que segue que $A \in \mathcal{C B}_{\mathcal{E}}$. Por outro lado, se $A \subseteq X$ for infinito, então, dado $x \in A$, podemos tomar uma sequência $\left(y_{n}\right)_{n \in \mathbb{N}}$ de pontos de $A$ tal que, para todo $n \in \mathbb{N}, y_{n} \neq x$. Mas:

$$
y_{n} \neq x \quad \forall n \in \mathbb{N} \Rightarrow\left(x, y_{n}\right) \in(A \times A) \backslash \Delta \quad \forall n \in \mathbb{N} \Rightarrow(A \times A) \backslash \Delta \text { é infinito. }
$$

E, sendo assim, $A \times A \notin \mathcal{E}$.

f) Sejam $\tau$ uma topologia sobre $X$ e

$$
\mathcal{E}_{i}:=\{E \subseteq X \times X: E \text { é próprio em }(X, \tau)\}
$$

a estrutura coarse indiscreta associada a $\tau$. Antes de mais nada, recordemos que um conjunto $E \subseteq X \times X$ é próprio em $(X, \tau)$ se, e somente se, $E[K]$ e $E^{-1}[K]$ forem relativamente compactos em $(X, \tau)$ sempre que $K \subseteq X$ for relativamente compacto em $(X, \tau)$. Dito isso, observe que, para todos $A, K \subseteq X$, temos que

$$
(A \times A)^{-1}[K]=(A \times A)[K]=\left\{\begin{array}{ll}
\emptyset, & \text { se } A \cap K=\emptyset \\
A, & \text { caso contrário }
\end{array} .\right.
$$

Assim sendo, fica claro que, dado $A \subseteq X, A \times A$ será próprio em $(X, \tau)$ se, e somente se, $A$ for relativamente compacto em $(X, \tau)$. E, portanto,

$$
\mathcal{C B}_{\mathcal{E}_{i}}=\{A \subseteq X: A \text { é relativamente compacto em }(X, \tau)\} .
$$

A seguir, vamos caracterizar os conjuntos grosseiramente limitados em $\left(G, \mathcal{E}_{L}\right)$, em que $(G, \cdot, \tau)$ é um grupo topológico e $\mathcal{E}_{L}$ é a sua estrutura coarse à esquerda, bem como estabelecer uma condição suficiente para que um conjunto $A \subseteq G$ seja grosseiramente limitado em $\left(G, \mathcal{E}_{L}\right)$. No entanto, antes que possamos fazer isso, precisaremos enunciar alguns resultados preliminares, o primeiro dos quais foi demonstrado por G. Birkhoff em um artigo de 1936 [Bir36]. Por se tratar de um resultado já há muito estabelecido, não faremos, aqui, sua demonstração, a qual, entretanto, pode ser facilmente compreendida a partir da leitura do trabalho de Birkhoff.

Lema 6.2.4. Sejam $(G, \cdot, \tau)$ um grupo topológico, e $\in G$ o elemento neutro de $(G, \cdot)$ e $\left(V_{n}\right)_{n \in \mathbb{Z}}$ uma familia de vizinhanças abertas e simétricas de e tal que $G=\bigcup_{n \in \mathbb{Z}} V_{n}$ e $V_{n}^{3} \subseteq V_{n+1}$ para todo 
$n \in \mathbb{Z}$. Nessas condições, dadas $\delta, d: G \times G \longrightarrow \mathbb{R}$ tais que

$$
\delta(x, y)=\inf \left\{2^{n}: n \in \mathbb{Z} e x^{-1} y \in V_{n}\right\}
$$

$e$

$$
d(x, y)=\inf \left\{\sum_{i=0}^{k-1} \delta\left(h_{i}, h_{i+1}\right): k \in \mathbb{N}, h_{0}, \ldots, h_{k} \in G, h_{0}=x \text { e } h_{k}=y\right\},
$$

temos que d é uma pseudométrica contínua e invariante à esquerda sobre $G$, e que, para todos $x, y \in G$,

$$
\frac{1}{2} \delta(x, y) \leq d(x, y) \leq \delta(x, y)
$$

LEMA 6.2.5. Sejam $(G, \cdot, \tau)$ um grupo topológico, e $\in G$ o elemento neutro de $(G, \cdot)$ e $W \subseteq G$ uma vizinhança aberta de e em $(G, \tau)$. Nessas condições, existe uma sequência $\left(V_{n}\right)_{n \in \mathbb{N}_{0}}$ de vizinhanças abertas de e tal que $\left(V_{n+1}\right)^{3} \subseteq V_{n} \subseteq V_{0}=W$ para todo $n \in \mathbb{N}_{0}$.

Demonstração. Vamos construir a sequência $\left(V_{n}\right)_{n \in \mathbb{N}_{0}}$ por recursão. Para começar, definamos $V_{0}:=W$. Tomemos, agora, $n \in \mathbb{N}_{0}$, e vamos mostrar como construir $V_{n+1}$ a partir de $V_{n}$.

Seja $f: G \times G \times G \longrightarrow G$ tal que $f(x, y, z)=x y z$. Observe que $f=m \circ \alpha$, em que

$$
\begin{aligned}
m: G \times G & \longrightarrow G \\
(x, y) & \longmapsto x y
\end{aligned}
$$

e

$$
\begin{aligned}
\alpha: G \times G \times G & \longrightarrow G \times G \\
(x, y, z) & \longmapsto(x, m(y, z)) .
\end{aligned}
$$

Sendo $(G, \cdot, \tau)$ um grupo topológico, temos, por hipótese, que $m$ é contínua. E, a partir disso, não é difícil mostrar que $\alpha$ e, por conseguinte, $f$, são, também, contínuas. No que segue, utilizaremos fortemente a continuidade de $f$.

Com efeito, como $f$ é contínua, $f(e, e, e)=e, e \in V_{n}, V_{n} \in \tau$ e

$$
\mathscr{B}:=\left\{U_{1} \times U_{2} \times U_{3}: U_{1}, U_{2}, U_{3} \in \tau\right\}
$$

é uma base da topologia produto induzida por $\tau$ sobre $G \times G \times G$, existem $U_{1}, U_{2}, U_{3} \in \tau$ tais que $(e, e, e) \in U_{1} \times U_{2} \times U_{3}$ e $f\left(U_{1} \times U_{2} \times U_{3}\right) \subseteq V_{n}$. Seja $U:=U_{1} \cap U_{2} \cap U_{3}$. Observe que $U \in \tau, e \in U \mathrm{e}$

$$
U^{3}=f(U \times U \times U) \subseteq f\left(U_{1} \times U_{2} \times U_{3}\right) \subseteq V_{n} \underset{(*)}{\subseteq} W
$$

Assim sendo, basta tomarmos $V_{n+1}:=U$.

$[(*)$ Por construção.]

LEMA 6.2.6. Sejam $(G, \cdot, \tau)$ um grupo topológico, e $\in G$ o elemento neutro de $(G, \cdot)$ e $\left(W_{n}\right)_{n \in \mathbb{Z}}$ uma família de vizinhanças abertas de e tal que $G=\bigcup_{n \in \mathbb{Z}} W_{n}$ e $W_{n}{ }^{3} \subseteq W_{n+1}$ para todo $n \in \mathbb{Z}$. Nessas condições, $\left(V_{n}\right)_{n \in \mathbb{Z}}$, em que, para cada $n \in \mathbb{Z}, V_{n}:=W_{n} \cap W_{n}^{-1}$, é uma família de vizinhanças abertas e simétricas de e tal que $G=\bigcup_{n \in \mathbb{Z}} V_{n}$ e, para cada $n \in \mathbb{Z}, V_{n}^{3} \subseteq V_{n+1}$.

Demonstração. Seja $n \in \mathbb{Z}$ dado. Inicialmente, observe que

$$
\left(W_{n}^{-1}\right)^{3}=\left(W_{n}^{3}\right)^{-1} \subseteq\left(W_{n+1}\right)^{-1} .
$$


Disso concluímos que

$$
V_{n}^{3}=\left(W_{n} \cap W_{n}^{-1}\right)^{3} \subseteq W_{n}^{3} \cap\left(W_{n}^{-1}\right)^{3} \subseteq W_{n+1} \cap\left(W_{n+1}\right)^{-1}=V_{n+1}
$$

Mostremos, agora, que $V_{n}$ é uma vizinhança aberta de $e$. Para isso, consideremos a função

$$
\begin{aligned}
i: G & \longrightarrow G \\
x & \longmapsto x^{-1} .
\end{aligned}
$$

Como $(G, \cdot, \tau)$ é um grupo topológico, temos, por hipótese, que $i$ é contínua. Além disso, é fácil ver que $i$ é inversível e que $i^{-1}=i$. Dessa forma, vale que

$$
W_{n}^{-1}=i\left(W_{n}\right)=i^{-1}\left(W_{n}\right) .
$$

Como $i$ é contínua e $W_{n} \in \tau$, isso prova que $W_{n}{ }^{-1}$ também pertence a $\tau$. E, de $e \in V_{n}$ e de $W_{n}, W_{n}^{-1} \in \tau$, concluímos que $V_{n}$ é uma vizinhança aberta de $e$ em $(G, \tau)$.

Também não é difícil ver que $V_{n}$ é simétrica, bastando, para isso, notar que

$$
V_{n}^{-1}=i\left(V_{n}\right)=i\left(W_{n} \cap W_{n}^{-1}\right)=i\left(W_{n}\right) \cap i\left(W_{n}^{-1}\right)=W_{n}^{-1} \cap W_{n}=V_{n},
$$

sendo a terceira igualdade uma consequência da injetividade de $i$.

Como $n \in \mathbb{Z}$ é arbitrário, disso resulta que $\left(V_{n}\right)_{n \in \mathbb{Z}}$ é uma família de vizinhanças abertas e simétricas de em $(G, \tau)$. Por fim, vamos mostrar que $G=\bigcup_{n \in \mathbb{Z}} V_{n}$. Para isso, é suficiente provarmos que cada $x \in G$ pertence a $V_{n}$ para algum $n \in \mathbb{Z}$.

Comecemos tomando $x \in G$ de modo arbitrário. Como $G=\bigcup_{n \in \mathbb{Z}} W_{n}$, existem $n, m \in \mathbb{Z}$ tais que $x \in W_{n}$ e $x^{-1} \in W_{m}$. Seja $N:=\max \{n, m\}$. Observe que $W_{n} \cup W_{m} \subseteq W_{N}$, uma vez que, por hipótese, temos que

$$
W_{k} \underset{e \in W_{k}}{\subseteq} W_{k}^{3} \subseteq W_{k+1} \quad \forall k \in \mathbb{Z}
$$

E, de $x, x^{-1} \in W_{N}$, concluímos que $x \in W_{N} \cap W_{N}^{-1}=V_{N}$.

Uma vez munidos desses resultados auxiliares, podemos, por fim, demonstrar um dos principais resultados desta seção. Antes disso, porém, faremos uma pequena observação sobre notação.

NotaÇÃo: No caso especial em que $(G, \cdot, \tau)$ é um grupo topológico, denotaremos a coleção dos conjuntos grosseiramente limitados em $\left(G, \mathcal{E}_{L}\right)$ por $\mathcal{O B} .^{4}$

Proposição 6.2.7. Sejam $(G, \cdot, \tau)$ um grupo topológico, e $\in G$ o elemento neutro de $(G, \cdot), \mathcal{D}$ a coleção das pseudométricas sobre $G$ que são continuas e invariantes à esquerda, e $\mathcal{E}_{L}:=\bigcap_{d \in \mathcal{D}} \mathcal{E}_{d}$ a estrutura coarse à esquerda associada a $(G, \cdot, \tau)$. Nessas condições, dado $A \subseteq G$, temos que

$$
(4) \underset{(*)}{\Rightarrow}(3) \Leftrightarrow(2) \Leftrightarrow(1)
$$

\footnotetext{
${ }^{4} \mathrm{~A}$ notação $\mathcal{O B}$ remete ao termo orbits bounded, e está relacionada ao fato (o qual não exploraremos aqui) de que um subconjunto $A \subseteq G$ é grosseiramente limitado em $\left(G, \mathcal{E}_{L}\right)$ se, e somente se, $A \star x$ possui diâmetro finito para todo $x \in X$ e toda ação isométrica e contínua $\star: G \times X \longrightarrow X$ de $(G, \cdot)$ sobre um espaço métrico $(X, d)$, em que, para cada $x \in X$,

$$
A \star x:=\{g \star x: g \in A\}
$$

denota a $A$-órbita de $x$ em relação a $\star$. Para mais detalhes, veja [Ros17a].
} 
em que:

(1) A é grosseiramente limitado em $\left(G, \mathcal{E}_{L}\right)$;

(2) $\operatorname{diam}_{d}(A)$ é finito para toda $d \in \mathcal{D}$;

(3) Para toda sequência $\left(V_{n}\right)_{n \in \mathbb{N}}$ de subconjuntos abertos de $(G, \tau)$ tal que $G=\bigcup_{n \in \mathbb{N}} V_{n}$ e, para todo $n \in \mathbb{N}, V_{n}, V_{n}^{2} \subseteq V_{n+1}$, existe $n \in \mathbb{N}$ tal que $A \subseteq V_{n} ;$

(4) Para toda vizinhança $V$ de e em $(G, \tau)$, existem $k \in \mathbb{N}$ e um conjunto finito $F \subseteq G$ tais que $A \subseteq(F V)^{k}$.

$(*)$ Observe que, em geral, $(3) \nRightarrow(4)$. Contudo, pode-se mostrar que isso ocorre no caso particular em que, para cada vizinhança $V$ de $e$, existe um conjunto finito ou enumerável $C \subseteq G$ tal que $V \cup C$ é um conjunto gerador de $(G, \cdot)$. Para mais detalhes, veja [Ros17a].

Demonstração. (4) $\Rightarrow(3)$

Seja $\left(V_{n}\right)_{n \in \mathbb{N}}$ uma sequência como em (3). Como $G=\bigcup_{n \in \mathbb{N}} V_{n}$, temos, em particular, que existe $m \in \mathbb{N}$ tal que $e \in V_{m}$ Por sua vez, sendo $V_{m}$ uma vizinhança de $e$, segue de (5) que existem $k \in \mathbb{N}$ e $F \subseteq G$ finito tais que $A \subseteq\left(F V_{m}\right)^{k}$. E, como $F$ é finito, $G=\bigcup_{n \in \mathbb{N}} V_{n}$ e $V_{n} \subseteq V_{n+1}$ para todo $n \in \mathbb{N}$, podemos fixar $n \in\{m, m+1, \ldots\}$ de modo que $F \subseteq V_{n}$. Observe que

$$
A \subseteq\left(F V_{m}\right)^{k} \subseteq\left(V_{n} V_{m}\right)^{k} \subseteq\left(V_{n}^{2}\right)^{k} \subseteq\left(V_{n+1}\right)^{k}
$$

Dessa forma, se tomarmos $j \in \mathbb{N}$ tal que $2^{j} \geq k$, então, como $e \in V_{n+1}$, teremos que $\left(V_{n+1}\right)^{k} \subseteq$ $\left(V_{n+1}\right)^{2^{j}}$, do que segue que

$$
A \subseteq\left(V_{n+1}\right)^{k} \subseteq\left(V_{n+1}\right)^{2^{j}} \subseteq V_{n+j+1}
$$

$(3) \Rightarrow(2)$

Seja $d \in \mathcal{D}$ dada e, para cada $n \in \mathbb{N}$, definamos

$$
V_{n}:=\left\{x \in G: d(e, x)<2^{n}\right\}=B_{G}^{d}\left(e, 2^{n}\right) .
$$

Dado $n \in \mathbb{N}$, observe que

$$
V_{n}:=\left\{x \in G: d(e, x)<2^{n}\right\} \subseteq\left\{x \in G: d(e, x)<2^{n+1}\right\}=V_{n+1} .
$$

Além disso, como $d$ é invariante à esquerda em $(G, \cdot)$, é fácil ver que

$$
d(e, x y) \leq d(e, x)+d(x, x y)=d(e, x)+d(e, y)<2^{n}+2^{n}=2^{n+1}
$$

para todos $x, y \in V_{n}$, do que segue que $V_{n}^{2} \subseteq V_{n+1}$.

Vamos, agora, provar que $V_{n} \in \tau$. Para isso, comecemos fixando $x \in V_{n}$ de modo arbitrário e notando que

$$
\left.x \in V_{n} \Rightarrow d(e, x)<2^{n} \Rightarrow d(e, x) \in\right]-\infty, 2^{n}[.
$$

Como $]-\infty, 2^{n}\left[\in \tau_{\mathbb{R}}, d\right.$ é contínua em $(e, x)$ e

$$
\mathscr{B}:=\left\{W_{1} \times W_{2}: W_{1}, W_{2} \in \tau\right\}
$$


é base da topologia produto induzida por $\tau$ sobre $G \times G$, disso resulta que existem $W_{1}, W_{2} \in \tau$ tais que $(e, x) \in W_{1} \times W_{2}$ e $d\left(x^{\prime}, y^{\prime}\right)<2^{n}$ para todo $\left(x^{\prime}, y^{\prime}\right) \in W_{1} \times W_{2}$. E, como $e \in W_{1}$, isso implica, em particular, que $d(e, y)<2^{n}$ para todo $y \in W_{2}$, do que segue que $W_{2} \subseteq V_{n}$. Mas:

$$
\left(W_{2} \in \tau \text { e } x \in W_{2} \subseteq V_{n}\right) \Rightarrow x \in \operatorname{int}_{\tau}\left(V_{n}\right)
$$

E, sendo $x \in V_{n}$ arbitrário, disso concluímos que $V_{n}$ é aberto em $(G, \tau)$.

Por fim, note que

$$
\begin{aligned}
\lim _{n \rightarrow+\infty} 2^{n}=+\infty & \Rightarrow \forall x \in G, \exists n \in \mathbb{N} \text { t.q. } d(e, x)<2^{n} \\
& \Rightarrow \forall x \in G, \exists n \in \mathbb{N} \text { t.q. } x \in V_{n} \Rightarrow G=\bigcup_{n \in \mathbb{N}} V_{n} .
\end{aligned}
$$

Com isso, mostramos que $\left(V_{n}\right)_{n \in \mathbb{N}}$ satisfaz todas as hipóteses de (3). Assim sendo, segue de (3) que existe $n \in \mathbb{N}$ tal que $A \subseteq V_{n}$. Disso resulta, em particular, que

$$
d(x, y) \leq d(x, e)+d(e, y)<2^{n}+2^{n}=2^{n+1}
$$

para todo $(x, y) \in A \times A$, a partir do que concluímos que diam ${ }_{d}(A) \leq 2^{n+1}$ e, portanto, é finito.

$(2) \Rightarrow(3)$

Suponhamos, por absurdo, que exista uma sequência $\left(W_{n}\right)_{n \in \mathbb{N}}$ como em (3) tal que $A \nsubseteq W_{n}$ para nenhum $n \in \mathbb{N}$. Note que, como $G=\bigcup_{n \in \mathbb{N}} W_{n}$, podemos supor, sem perda de generalidade, que $e \in W_{1}$. E, nesse caso, segue do lema 6.2.5 que existe uma sequência $\left(U_{n}^{\prime}\right)_{n \in \mathbb{N}_{0}}$ de vizinhanças abertas de $e$ tal que $\left(U_{n+1}^{\prime}\right)^{3} \subseteq U_{n}^{\prime} \subseteq U_{0}^{\prime}=W_{1}$ para todo $n \in \mathbb{N}_{0}$.

Consideremos, agora, a família $\left(U_{n}\right)_{n \in \mathbb{Z}}$ tal que, para cada $n \in \mathbb{Z}$,

$$
U_{n}:=\left\{\begin{array}{ll}
U_{-n}^{\prime}, & \text { se } n \leq 0 \\
W_{2 n+1}, & \text { se } n \geq 0
\end{array} .\right.
$$

Observe que, para cada $n \in \mathbb{Z}, U_{n}$ é uma vizinhança aberta de e em $(G, \tau)$. Além disso, uma vez que, por construção, $U_{n} \subseteq U_{0}=W_{1}$ para todo $n \leq 0$ e $U_{n} \subseteq U_{n+1}$ para todo $n \geq 0$, é fácil ver que

$$
G=\bigcup_{n \in \mathbb{N}} W_{n}=\bigcup_{n \in \mathbb{N}_{0}} W_{2 n+1}=\bigcup_{n \in \mathbb{N}_{0}} U_{n}=\bigcup_{n \in \mathbb{Z}} U_{n} .
$$

A seguir, vamos mostrar que $U_{n}{ }^{3} \subseteq U_{n+1}$ para todo $n \in \mathbb{Z}$. Faremos isso em duas etapas. Inicialmente, observe que, por hipótese,

$$
\left(U_{n}^{\prime}\right)^{3} \subseteq U_{n-1}^{\prime} \subseteq U_{0}^{\prime}
$$

para todo $n \in \mathbb{N}$. Assim sendo, para todo $n \in \mathbb{Z}$ tal que $n<0$, vale que

$$
U_{n}^{3}=\left(U_{-n}^{\prime}\right)^{3} \subseteq U_{-n-1}^{\prime}=U_{-(n+1)}^{\prime}=U_{n+1} \subseteq U_{0}
$$


E, por outro lado, dado $n \in \mathbb{Z}$ tal que $n \geq 0$, temos, por definição, que

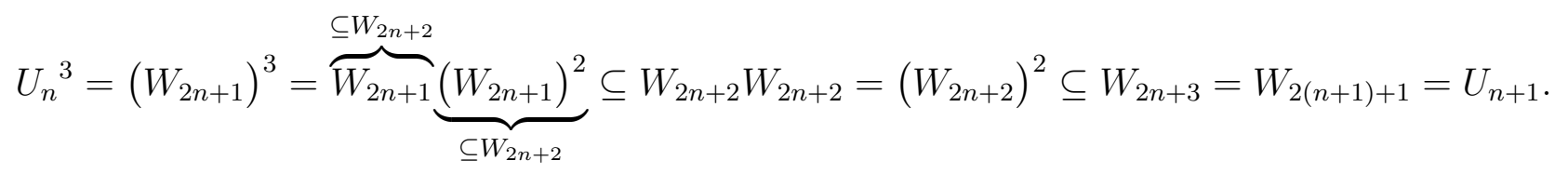

Dessa forma, segue do lema 6.2 .6 que $\left(V_{n}\right)_{n \in \mathbb{Z}}$, em que, para cada $n \in \mathbb{Z}, V_{n}=U_{n} \cap U_{n}{ }^{-1}$, é uma família de vizinhanças abertas e simétricas de $e$ tal que $G=\bigcup_{n \in \mathbb{Z}} V_{n}$ e, para cada $n \in \mathbb{Z}$, $V_{n}^{3} \subseteq V_{n+1}$. E, por sua vez, disso resulta, por 6.2 .4 , que a função $d: G \times G \longrightarrow \mathbb{R}$ definida de modo que

$$
d(x, y)=\inf \left\{\sum_{i=0}^{k-1} \delta\left(h_{i}, h_{i+1}\right): k \in \mathbb{N}, h_{0}, \ldots, h_{k} \in G, h_{0}=x \text { e } h_{k}=y\right\}
$$

em que $\delta: G \times G \longrightarrow \mathbb{R}$ é tal que $\delta(x, y)=\inf \left\{2^{n}: n \in \mathbb{Z}\right.$ e $\left.x^{-1} y \in V_{n}\right\}$, é uma pseudométrica contínua e invariante à esquerda sobre $G$ tal que

$$
\frac{1}{2} \delta(x, y) \leq d(x, y) \leq \delta(x, y)
$$

para todos $x, y \in G$.

Observe que, para todo $n \in \mathbb{Z}$ e todo $x \in G$,

$$
\begin{aligned}
x \in B_{G}^{d}\left(e, 2^{n}\right) & \Rightarrow d(e, x)<2^{n} \Rightarrow \delta(e, x)<2^{n+1} \Rightarrow \inf \left\{2^{j}: j \in \mathbb{Z} \text { e } x \in V_{j}\right\}<2^{n+1} \\
& \Rightarrow \exists j \in \mathbb{Z} \text { t.q. } x \in V_{j} \text { e } 2^{j}<2^{n+1} \\
& \Rightarrow \exists j \in \mathbb{Z} \text { t.q. } x \in V_{j} \text { e } j<n+1 \\
& \Rightarrow x \in V_{n+1},
\end{aligned}
$$

uma vez que, por hipótese, $V_{j} \subseteq V_{n+1}$ para todo $j \in \mathbb{Z}$ tal que $j \leq n+1$. Disso resulta que $B_{G}^{d}\left(e, 2^{n}\right) \subseteq V_{n+1}$ para todo $n \in \mathbb{Z}$.

Por outro lado, tendo em vista que, para todo $n \in \mathbb{N}, A \nsubseteq W_{n}$ e $V_{n} \subseteq U_{n}=W_{2 n+1}$, é imediato ver que $A \nsubseteq V_{n}$ para nenhum $n \in \mathbb{N}$, a partir do que concluímos que $A \nsubseteq B_{G}^{d}\left(e, 2^{n}\right)$ para nenhum $n \in \mathbb{N}$ e, portanto, $\operatorname{diam}_{d}(A)=+\infty$. Isso, porém, é uma contradição, haja visto que $d \in \mathcal{D}$ e, por hipótese, $\operatorname{diam}_{d^{\prime}}(A)$ é finito para toda pseudométrica $d^{\prime} \in \mathcal{D}$.

$(2) \Leftrightarrow(1)$

Note que, pelo exemplo 6.2.3-d), temos que, para cada $d \in \mathcal{D}$,

$$
\mathcal{C B}_{\mathcal{E}_{d}}=\left\{B \subseteq G: \operatorname{diam}_{d}(B) \text { é finito }\right\}
$$

Disso resulta que

$$
A \times A \in \mathcal{E}_{L} \Leftrightarrow A \times A \in \bigcap_{d \in \mathcal{D}} \mathcal{E}_{d} \Leftrightarrow A \times A \in \mathcal{E}_{d} \quad \forall d \in \mathcal{D} \Leftrightarrow \operatorname{diam}_{d}(A)<+\infty \quad \forall d \in \mathcal{D},
$$

a partir do que segue, imediatamente, que $A$ será grosseiramente limitado em $\left(G, \mathcal{E}_{L}\right)$ se, e somente se, possuir diâmetro finito em relação a toda pseudométrica em $\mathcal{D}$.

Podemos, agora, utilizar a proposição 6.2.7 para deduzir algumas das propriedades de $\mathcal{O B}$. 
Proposição 6.2.8. Sejam $(G, \cdot, \tau)$ um grupo topológico, e $\in G$ o elemento neutro de $(G, \cdot)$ e $\mathcal{E}_{L}$ a estrutura coarse à esquerda associada a $(G, \cdot, \tau)$. Nessas condições, a coleção $\mathcal{O} \mathcal{B}$ dos conjuntos grosseiramente limitados em $\left(G, \mathcal{E}_{L}\right)$ é um ideal sobre $G$ tal que $\{e\} \in \mathcal{O B} e$ $\bar{A}, A^{-1}, A B \in \mathcal{O B}$ para todos $A, B \in \mathcal{O B}$.

Demonstração. Inicialmente, observe que

$$
\left(\{(e, e)\} \subseteq \Delta \text { e } \Delta \in \mathcal{E}_{L}\right) \Rightarrow\{(e, e)\} \in \mathcal{E}_{L} \Rightarrow\{e\} \times\{e\} \in \mathcal{E}_{L} \Rightarrow\{e\} \in \mathcal{O B}
$$

Além disso, segue diretamente da observação 6.2 .2 que, para todos $A, B \subseteq G$,

$$
(A \in \mathcal{O B} \text { e } B \subseteq A) \Rightarrow B \in \mathcal{O B}
$$

Para provarmos que $\mathcal{O B}$ possui as demais propriedades do enunciado, utilizaremos a proposição 6.2.7.

Para começar, tomemos $A, B \in \mathcal{O B}$ de modo arbitrário, e consideremos uma sequência $\left(V_{n}\right)_{n \in \mathbb{N}}$ de subconjuntos abertos de $G$ tal que $G=\bigcup_{n \in \mathbb{N}} V_{n}$ e, para todo $n \in \mathbb{N}, V_{n}, V_{n}^{2} \subseteq V_{n+1}$. Como $A, B \in \mathcal{O B}$ e, para cada $n \in \mathbb{N}, V_{n} \subseteq V_{n+1}$, segue de 6.2 .7 que existe $n \in \mathbb{N}$ tal que $A, B \subseteq V_{n}$. Mas:

$$
A, B \subseteq V_{n} \Rightarrow A B \subseteq V_{n}^{2} \Rightarrow A B \subseteq V_{n+1} .
$$

E, assim sendo, podemos novamente utilizar a equivalência entre (1) e (3) na proposição 6.2.7 para concluir que $A B \in \mathcal{O B}$.

Fixemos, agora, uma pseudométrica $d \in \mathcal{D}$ qualquer. Como $A, B \in \mathcal{O B}$, segue de 6.2 .7 que

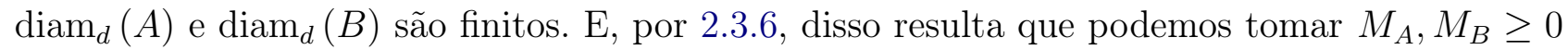
de modo que, para todo $x \in A$ e todo $y \in B$,

$$
d(e, x) \leq M_{A} \text { e } d(e, y) \leq M_{B}
$$

Dado $x \in G$, observe que

$$
x \in A \cup B \Rightarrow(x \in A \text { ou } x \in B) \Rightarrow\left(d(e, x) \leq M_{A} \text { ou } d(e, x) \leq M_{B}\right) \Rightarrow d(e, x) \leq M_{A}+M_{B} .
$$

E, sendo $d$ invariante à esquerda, também é fácil ver que

$$
x \in A^{-1} \Rightarrow x^{-1} \in A \Rightarrow d\left(e, x^{-1}\right) \leq M_{A} \Rightarrow d(x, e) \leq M_{A} \Rightarrow d(e, x) \leq M_{A} .
$$

Por fim, note ainda que

$$
x \in \bar{A} \Rightarrow \forall n \in \mathbb{N}, \exists x_{n} \in A \text { t.q. } d\left(x_{n}, x\right)<\frac{1}{n} .
$$

Mas, para todo $x^{\prime} \in A$,

$$
d\left(x^{\prime}, x\right)<\frac{1}{n} \Rightarrow d(e, x) \leq d\left(e, x^{\prime}\right)+d\left(x^{\prime}, x\right)<M_{A}+\frac{1}{n} .
$$

E, assim sendo,

$$
x \in \bar{A} \Rightarrow d(e, x)<M_{A}+\frac{1}{n} \quad \forall n \in \mathbb{N} \Rightarrow d(e, x) \leq M_{A}
$$

Como $x \in G$ e $d \in \mathcal{D}$ são arbitrários, disso concluímos, por 2.3.6, que $\operatorname{diam}_{d}(A \cup B), \operatorname{diam}_{d}(\bar{A})$ 
e $\operatorname{diam}_{d}\left(A^{-1}\right)$ são finitos para toda $d \in \mathcal{D} .^{5} \mathrm{E}$, sendo assim, segue de 6.2 .7 que $A \cup B, \bar{A}$ e $A^{-1}$ pertencem a $\mathcal{O B}$.

Seja $(G, \cdot, \tau)$ um grupo topológico. A proposição 6.2 .8 nos mostra que a coleção $\mathcal{O B}$ dos conjuntos grosseiramente limitados em $\left(G, \mathcal{E}_{L}\right)$ é um ideal sobre $G$ nas condições do teorema 6.1.11. Assim sendo, segue de 6.1.11 que

$$
\mathcal{E}_{\mathcal{O B}}:=\left\{E \subseteq G \times G: \exists A \in \mathcal{O B} \text { t.q. } E \subseteq E_{A}\right\}
$$

é uma estrutura coarse sobre $G$ invariante à esquerda em $(G, \cdot)$. Tendo em vista a definição de $\mathcal{O B}$, é natural esperar que uma tal estrutura coarse esteja, de alguma forma, relacionada com a estrutura coarse à esquerda $\mathcal{E}_{L}$. E ela, de fato, está. Na verdade, veremos, a seguir, que $\mathcal{E}_{\mathcal{O B}}=\mathcal{E}_{L}$

Proposição 6.2.9. Para todo grupo topológico $(G, \cdot, \tau)$, temos que $\mathcal{E}_{\mathcal{O B}}=\mathcal{E}_{L}$.

Demonstração. Sejam $(G, \cdot, \tau)$ um grupo topológico e $e \in G$ o elemento neutro de $(G, \cdot)$.

Para começar, tomemos $E \in \mathcal{E}_{\mathcal{O B}}$ de modo arbitrário e fixemos $A \in \mathcal{O B}$ tal que $E \subseteq E_{A}$. Como $A \in \mathcal{O B}$, segue da proposição 6.2 .7 que $\operatorname{diam}_{d}(A)$ é finito para toda $d \in \mathcal{D}$. E, pela proposição 2.3.6, disso resulta que, para cada $d \in \mathcal{D}$, existe $\alpha_{d} \geq 0$ tal que $d(x, y) \leq \alpha_{d}$ para todos $x, y \in A$.

Seja $d \in \mathcal{D}$ uma pseudométrica qualquer. Como $d$ é invariante à esquerda, é fácil ver que, para todo $(x, y) \in G \times G$,

$$
(x, y) \in E_{A} \Rightarrow x^{-1} y \in A \Rightarrow d\left(e, x^{-1} y\right) \leq \alpha_{d} \Rightarrow d(x, y) \leq \alpha_{d} \Rightarrow(x, y) \in E_{\alpha_{d}}^{d} .
$$

Assim sendo, $E \subseteq E_{A} \subseteq E_{\alpha_{d}}^{d}$. Mas:

$$
\left(E \subseteq E_{\alpha_{d}}^{d} \text { e } E_{\alpha_{d}}^{d} \in \mathcal{E}_{d}\right) \Rightarrow E \in \mathcal{E}_{d}
$$

E, como $d \in \mathcal{D}$ é arbitrária, disso concluímos que $E \in \mathcal{E}_{L}$.

Tomemos, agora, $E^{\prime} \in \mathcal{E}_{L}$ qualquer, e vamos mostrar que $E^{\prime} \in \mathcal{E}_{\mathcal{O B}}$. Para isso, notemos, inicialmente, que, como $E^{\prime} \in \mathcal{E}_{L}$ e $\mathcal{E}_{L}$ é invariante à esquerda (veja o exemplo 6.1.5), podemos tomar $F \in \mathcal{E}_{L}$ invariante à esquerda tal que que $E^{\prime} \subseteq F$. Observe que

$$
F \in \mathcal{E}_{L} \Rightarrow F \in \mathcal{E}_{d} \quad \forall d \in \mathcal{D} \Rightarrow\{d(x, y):(x, y) \in F\} \text { é limitado } \forall d \in \mathcal{D} .
$$

Assim sendo, para cada $d \in \mathcal{D}$, podemos, também, fixar $M_{d}>0$ de modo que $d(x, y)<M_{d}$ para todo $(x, y) \in F$. Feito isso, observe que, para todos $x, y \in G$ e para toda $d \in \mathcal{D}$,

$$
\begin{aligned}
x, y \in A_{F} & \Rightarrow(e, x),(e, y) \in F \Rightarrow d(e, x), d(e, y)<M_{d} \\
& \Rightarrow d(x, y) \leq d(e, x)+d(e, y)<2 M_{d} .
\end{aligned}
$$

Isso mostra que $\operatorname{diam}_{d}\left(A_{F}\right)$ é finito para toda $d \in \mathcal{D}$, a partir do que segue de 6.2 .7 que $A_{F} \in \mathcal{O B}$. E, uma vez que $E^{\prime} \subseteq F=E_{A_{F}}$, disso concluímos que $E^{\prime} \in \mathcal{E}_{\mathcal{O B}}$.

No capítulo 3, mostramos que, para todo grupo topológico $(G, \cdot, \tau)$ e toda pseudométrica invariante à esquerda $d$ sobre $G$, temos que

$$
\mathcal{U}_{L}=\mathscr{D}_{d} \Leftrightarrow \tau=\tau_{d}
$$

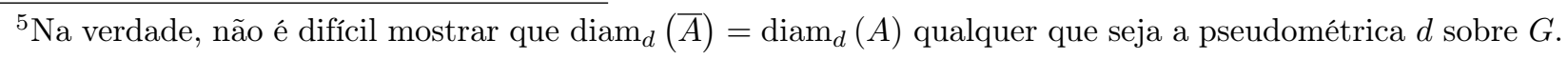


No que segue, faremos o mesmo tipo de estudo em relação à estrutura coarse à esquerda de um grupo topológico. Mais especificamente, vamos mostrar que uma pseudométrica contínua e invariante à esquerda $d$ em um grupo topológico $(G, \cdot, \tau)$ induz a estrutura coarse à esquerda $\mathcal{E}_{L}$ sobre $G$ se, e somente se, $\left(G, \mathcal{E}_{d}\right)$ e $\left(G, \mathcal{E}_{L}\right)$ possuírem os mesmos conjuntos grosseiramente limitados.

TeOrema 6.2.10. Sejam $(G, \cdot, \tau)$ um grupo topológico, $e \in G$ o elemento neutro de $(G, \cdot)$ e d uma pseudométrica contínua e invariante à esquerda. Nessas condições,

$$
\mathcal{E}_{L}=\mathcal{E}_{d} \Leftrightarrow \mathcal{O B}=\mathcal{C B}_{\mathcal{E}_{d}} \Leftrightarrow \mathcal{O B}=\left\{A \subseteq G: \operatorname{diam}_{d}(A)<+\infty\right\}
$$

Demonstração. A segunda equivalência é trivial, uma vez que, no exemplo 6.2.3-d), já mostramos que

$$
\mathcal{C B}_{\mathcal{E}_{d}}=\left\{A \subseteq G: \operatorname{diam}_{d}(A)<+\infty\right\}
$$

E, sendo assim, basta provarmos que

$$
\mathcal{E}_{L}=\mathcal{E}_{d} \Leftrightarrow \mathcal{O B}=\left\{A \subseteq G: \operatorname{diam}_{d}(A)<+\infty\right\} .
$$

$(\Rightarrow)$ Com efeito,

$$
\mathcal{E}_{L}=\mathcal{E}_{d} \Rightarrow \mathcal{O B}:=\mathcal{C B}_{\mathcal{E}_{L}}=\mathcal{C B}_{\mathcal{E}_{d}} \Rightarrow \mathcal{O B}=\left\{A \subseteq G: \operatorname{diam}_{d}(A)<+\infty\right\}
$$

$(\Leftarrow)$ Como, por hipótese, $d$ é contínua e invariante à esquerda, segue diretamente da definição de $\mathcal{E}_{L}$ que $\mathcal{E}_{L} \subseteq \mathcal{E}_{d}$

Mostremos, agora, a inclusão oposta. Para isso, comecemos tomando $E \in \mathcal{E}_{d}$ de modo arbitrário. Inicialmente, note que, sendo $\mathscr{B}_{d}:=\left\{E_{\alpha}^{d}: \alpha>0\right\}$ uma base de $\mathcal{E}_{d}$, podemos fixar $\alpha>0$ de modo que $E \subseteq E_{\alpha}^{d}$.

Por outro lado, como $\operatorname{diam}_{d}(B(e, \alpha))$ é finito, temos, por hipótese, que $B(e, \alpha)$ é grosseiramente limitado. E, sendo $d$ invariante à esquerda, temos também que

$$
\begin{aligned}
E_{\alpha}^{d} & :=\{(x, y) \in G \times G: d(x, y)<\alpha\}=\left\{(x, y) \in G \times G: d\left(e, x^{-1} y\right)<\alpha\right\} \\
& =\left\{(x, y) \in G \times G: x^{-1} y \in B(e, \alpha)\right\}=E_{B(e, \alpha)} .
\end{aligned}
$$

De $B(e, \alpha) \in \mathcal{O B}$ e de $E \subseteq E_{B(e, \alpha)}$, concluímos que $E \in \mathcal{E}_{\mathcal{O B}}$. Como $\mathcal{E}_{\mathcal{O B}}=\mathcal{E}_{L}$, isso prova que $E \in \mathcal{E}_{L}$. E, como $E \in \mathcal{E}_{d}$ é arbitrário, disso resulta que $\mathcal{E}_{d} \subseteq \mathcal{E}_{L}$.

Assim sendo, não nos resta alternativa senão concluir que $\mathcal{E}_{d}=\mathcal{E}_{L}$.

O teorema 6.2.10 é um resultado bastante interessante, e, na próxima seção, veremos uma de suas aplicações.

\subsection{A ESTRUTURA COARSE À ESQUERDA DO GRUPO ADITIVO DE UM ESPAÇO NORMADO}

Nesta última seção, focaremos nossa atenção no caso particular dos grupos aditivos de espaços normados. Nosso objetivo, aqui, é mostrar que a estrutura coarse à esquerda associada ao grupo aditivo de um espaço normado coincide com a estrutura coarse limitada induzida pela norma. Para tanto, precisaremos, inicialmente, fazer uma definição preliminar e, a partir dela, demonstrar um resultado auxiliar. 
DeFINIÇÃo 6.3.1. Seja $V$ um espaço vetorial sobre um corpo $\mathbb{K}$. Dados $n \in \mathbb{N}$ e $A_{1}, \ldots, A_{n} \subseteq$ $V$, denotaremos por $A_{1}+\ldots+A_{n}$ o conjunto

$$
\left\{v_{1}+\ldots+v_{n}: v_{i} \in A_{i} \forall i \in\{1, \ldots, n\}\right\} .
$$

No caso particular em que $A_{1}=\ldots=A_{n}=A$, utilizaremos a notação $n \star A$ para nos referirmos a $A_{1}+\ldots+A_{n}$.

LEMA 6.3.2. Seja $(X,\|\cdot\|)$ um espaço normado. Nessas condições, para todo $r>0$ e todo $n \in \mathbb{N}$, temos que

$$
n \star B\left(0_{X}, r\right):=\underbrace{B\left(0_{X}, r\right)+\ldots+B\left(0_{X}, r\right)}_{n \text { vezes }}=B\left(0_{X}, n \cdot r\right) .
$$

Demonstração. Sejam $r>0, n \in \mathbb{N}$ e $x \in n \star B\left(0_{X}, r\right)$ dados. Como $x \in n \star B\left(0_{X}, r\right)$, temos, por definição, que existem $x_{1}, \ldots, x_{n} \in B(0, r)$ tais que $x=x_{1}+\ldots+x_{n}$. E, por sua vez, disso segue que

$$
\|x\|=\left\|x_{1}+\ldots+x_{n}\right\| \leq \underbrace{\left\|x_{1}\right\|}_{<r}+\ldots+\underbrace{\left\|x_{n}\right\|}_{<r}<n \cdot r,
$$

o que prova que $x \in B\left(0_{X}, n \cdot r\right)$. Por fim, como $n \in \mathbb{N}$ e $x \in n \star B\left(0_{X}, r\right)$ são arbitrários, disso resulta que $n \star B\left(0_{X}, r\right) \subseteq B\left(0_{X}, n \cdot r\right)$ para todo $n \in \mathbb{N}$.

Provaremos a inclusão oposta por indução sobre $n$. O caso $n=1$ é óbvio. Suponhamos, agora, que $B\left(0_{X}, n \cdot r\right) \subseteq n \star B\left(0_{X}, r\right)$ para algum $n \in \mathbb{N}$, e vamos mostrar que, nesse caso, $B\left(0_{X},(n+1) \cdot r\right) \subseteq(n+1) \star B\left(0_{X}, r\right)$.

Para começar, fixemos $x \in B\left(0_{X},(n+1) \cdot r\right)$, e tomemos $x_{n+1}:=\frac{x}{n+1}$. Inicialmente, observe que

$$
\left\|x_{n+1}\right\|=\left\|\frac{x}{n+1}\right\|=\frac{\|x\|}{n+1}<r
$$

do que segue que $x_{n+1} \in B\left(0_{X}, r\right)$. Disso resulta, em particular, que

$$
\left\|x-x_{n+1}\right\|=\left\|(n+1) \cdot x_{n+1}-x_{n+1}\right\|=n \cdot\left\|x_{n+1}\right\|<n \cdot r,
$$

a partir do que concluímos que $x-x_{n+1} \in B\left(0_{X}, n \cdot r\right)$.

Mas, de $x-x_{n+1} \in B\left(0_{X}, n \cdot r\right)$, segue, por hipótese, que podemos tomar $x_{1}, \ldots, x_{n} \in$ $B\left(0_{X}, r\right)$ de tal modo que $x-x_{n+1}=x_{1}+\ldots+x_{n}$. E, de $x=x_{1}+\ldots+x_{n+1}$, em que $x_{1}, \ldots, x_{n+1} \in B\left(0_{X}, r\right)$, concluímos que $x \in(n+1) \star B\left(0_{X}, r\right)$. Como $x \in B\left(0_{X},(n+1) \cdot r\right)$ é completamente arbitrário, isso implica, imediatamente, o resultado desejado.

Antes de enunciarmos o próximo resultado, o qual é, em essência, um corolário do lema 6.3 .2 e da proposição 6.2.7, recordemos que, dado um espaço normado $(X,\|\cdot\|)$, dizemos que um um subconjunto $A \subseteq X$ é limitado em $(X,\|\cdot\|$ ) (ou, simplesmente, limitado) se existir $M \geq 0$ tal que $\|x\| \leq M$ para todo $x \in A$.

OBSERVAÇÃo 6.3.3. Não é difícil mostrar que um subconjunto $A \subseteq X$ é limitado em $(X,\|\cdot\|)$ se, e somente se, possui diâmetro finito em relação à métrica induzida pela norma. Com efeito, isso é uma consequência direta da proposição 2.3.6.

Proposição 6.3.4. Sejam $(X,\|\cdot\|)$ um espaço normado e $\mathcal{E}_{L}$ a estrutura coarse à esquerda associada a $\left(X,+, \tau_{\|\cdot\|}\right)$. Nessas condições, um subconjunto $A \subseteq X$ será grosseiramente limitado em $\left(X, \mathcal{E}_{L}\right)$ se, e somente se, for limitado. 
Demonstração. $(\Rightarrow)$ Seja $A \subseteq X$ um conjunto grosseiramente limitado em $\left(X, \mathcal{E}_{L}\right)$. Como a métrica induzida por $\|\cdot\|$ sobre $X$ é contínua e invariante à esquerda, segue da proposição 6.2 .7 que $\operatorname{diam}_{\|_{\|\cdot\|}}(A)$ é finito. E, pela proposição 2.3.6, disso resulta que existe $M \geq 0$ tal que $\|x\| \leq M$ para todo $x \in A$, a partir do que concluímos que $A$ é limitado.

$(\Leftarrow)$ Tomemos, agora, um conjunto limitado $B \subseteq X$, e consideremos uma vizinhança $V$ de $0_{X}$ em $\left(X, \tau_{\|\cdot\|}\right)$. Como $V$ é vizinhança de $0_{X}$, temos, por definição, que existe $\varepsilon>0$ tal que $B\left(0_{X}, \varepsilon\right) \subseteq V$. Observe que, sendo $B$ limitado, podemos fixar $n \in \mathbb{N}$ de modo que $B \subseteq$ $B\left(0_{X}, n \cdot \varepsilon\right)$. E, se assim o fizermos, seguirá de 6.3.2 que

$$
B \subseteq B\left(0_{X}, n \cdot \varepsilon\right)=\underbrace{B\left(0_{X}, \varepsilon\right)+\ldots+B\left(0_{X}, \varepsilon\right)}_{n \text { vezes }} \subseteq \underbrace{V+\ldots+V}_{n \text { vezes }}=n \star V .
$$

Por fim, sendo $V$ uma vizinhança arbitrária de $0_{X}$, decorre diretamente da proposição 6.2 .7 que $B$ é grosseiramente limitado em $\left(X, \mathcal{E}_{L}\right)$.

Estamos, agora, em condições de provar o último e mais importante resultado desta seção, o qual enunciaremos a seguir.

TEOREMA 6.3.5. A estrutura coarse à esquerda $\mathcal{E}_{L}$ associada ao grupo aditivo de um espaço normado $(X,\|\cdot\|)$ coincide com a estrutura coarse limitada induzida pela norma.

Demonstração. Isso é uma consequência imediata do teorema 6.2 .10 , da proposição 6.3 .4 e da observação 6.3.3.

Com efeito, a proposição 6.3.4, se utilizada em conjunto com a observação 6.3.3, permite-nos concluir que um subconjunto $A \subseteq X$ será grosseiramente limitado em $\left(X, \mathcal{E}_{L}\right)$ se, e somente se, possuir diâmetro finito em relação à métrica $d_{\|\cdot\|}$ induzida pela norma. E, por 2.7.29, disso segue que $\mathcal{E}_{d_{\|\cdot\|}}=\mathcal{E}_{L}$, uma vez que, como sabemos, $d_{\|\cdot\|}$ é contínua e invariante à esquerda. 


\section{Apêndice A}

\section{UNIFORMIDADES INVARIANTES À ESQUERDA}

Este apêndice tem como objetivo estudar as uniformidades invariantes à esquerda em grupos, à semelhança do que foi feito na primeira seção do capítulo 6 para a classe das estruturas coarse invariantes à esquerda.

A ideia, aqui, é explorar a dualidade entre os conceitos de uniformidade e de estrutura coarse para obter resultados análogos, no caso das uniformidades invariantes à esquerda, aos apresentados na seção 6.1 para as estruturas coarse invariantes à esquerda, e, a partir disso, estudar a relação entre a classe das uniformidades invariantes à esquerda em um grupo $(G, \cdot)$ e uma classe especial de filtros sobre $G$. O que queremos, com isso, é mostrar que, do mesmo modo como uma estrutura coarse invariante à esquerda sobre $G$ pode ser descrita em função de um ideal de $G$ e vice-versa, podemos também caracterizar uma uniformidade invariante à esquerda em termos de um certo filtro.

Uma vez munidos desses resultados, será possível estabelecer uma conexão muito bonita entre o material deste apêndice e o que vimos sobre grupos topológicos no capítulo 3. E, a partir disso, seremos capazes de entender com muito mais clareza o significado profundo por trás da estrutura uniforme à esquerda de um grupo topológico.

\section{A.1 UNIFORMIDADES INVARIANTES À ESQUERDA}

Nesta seção, vamos discutir alguns exemplos de uniformidades invariantes à esquerda em um grupo $(G, \cdot)$. E, para tanto, começaremos definindo o conceito de uniformidade invariante à esquerda.

DeFiniçÃo A.1.1 (Estrutura Uniforme inVARiante À ESQUERDA). Dado um grupo $(G, \cdot)$, dizemos que uma uniformidade $\mathscr{D}$ sobre $G$ é invariante à esquerda em $(G, \cdot)$ se $\mathscr{D}$ possuir uma base formada por conjuntos invariantes à esquerda em $(G, \cdot)$.

Observe a semelhança entre as definições de uniformidade e de estrutura coarse invariantes à esquerda. Essa semelhança, longe de ser mera coincidência, é uma consequência da enorme similaridade operacional entre uniformidades e estruturas coarse, a qual vem sendo enfatizada ao longo de todo o trabalho.

Vejamos, a seguir, alguns exemplos de uniformidades invariantes à esquerda.

Exemplos A.1.2. Nos itens a), b) e c), consideremos um grupo $(G, \cdot)$.

a) As uniformidades $\mathscr{D}_{1}:=\{G \times G\}$ e $\mathscr{D}_{2}:=\{D \subseteq G \times G: \Delta \subseteq D\}$ são ambas invariantes à esquerda em $(G, \cdot)$, pois possuem bases, $\mathscr{B}_{1}:=\{G \times G\}$ e $\mathscr{B}_{2}:=\{\Delta\}$, respectivamente, formadas por subconjuntos invariantes à esquerda. 
b) A uniformidade induzida por uma pseudométrica invariante à esquerda $d$ sobre $G$ é invariante à esquerda em $(G, \cdot)$, pois tem como base a coleção $\mathscr{B}_{d}:=\left\{D_{\varepsilon}^{d}: \varepsilon>0\right\}$, a qual é formada por subconjuntos invariantes à esquerda (veja o exemplo 6.1.2-b)) .

c) Sejam $(H, \cdot)$ um subgrupo de $(G, \cdot), \mathscr{D}_{G}$ uma uniformidade sobre $G$ e

$$
\mathscr{D}_{H}:=\left\{D \cap(H \times H): D \in \mathscr{D}_{G}\right\}
$$

a uniformidade induzida por $\mathscr{D}_{G}$ sobre $H$ (veja o exemplo 3.1.9). Nessas condições, se $\mathscr{D}_{G}$ for invariante à esquerda em $(G, \cdot)$, então $\mathscr{D}_{H}$ será invariante à esquerda em $(H, \cdot)$.

Para constatar isso, fixemos, inicialmente, uma base $\mathscr{B}_{G}$ de $\mathscr{D}_{G}$ formada por conjuntos invariantes à esquerda em $(G, \cdot)$. Observe que, sendo $\mathscr{B}_{G}$ uma base de $\mathscr{D}_{G}$, segue da observação 3.1 .10 que

$$
\mathscr{B}_{H}:=\left\{B \cap(H \times H): B \in \mathscr{B}_{G}\right\}
$$

é base de $\mathscr{D}_{H}$. E, sendo $(H, \cdot)$ um subgrupo de $(G, \cdot)$, podemos, facilmente, mostrar que $\mathscr{B}_{H}$ é formada por conjuntos invariantes à esquerda, bastando, para tanto, reproduzirmos o argumento utilizado no exemplo 6.1.2-c).

d) A estrutura uniforme à esquerda associada a um grupo topológico $(G, \cdot, \tau)$ é invariante à esquerda, pois tem como base a coleção $\mathscr{L}:=\left\{E_{V}: V \in \mathcal{V}\right\}$, em que, para cada $V \in \mathcal{V}$,

$$
E_{V}:=\left\{(x, y) \in G \times G: x^{-1} y \in V\right\}
$$

é invariante à esquerda em $(G, \cdot)$ (veja o comentário que sucede a definição 3.2.3).

\section{A.2 RelaCiOnANDo UNiformidades A FiLtros}

O nosso objetivo, a seguir, é mostrar que, dado um grupo $(G, \cdot)$, podemos estabelecer uma correspondência biunívoca entre a coleção das uniformidades sobre $G$ invariantes à esquerda em $(G, \cdot)$ e uma certa classe $\mathcal{F}$ de filtros sobre $G$. Para tanto, precisaremos, primeiramente, fazer uma definição preliminar. Antes disso, porém, recorde a definição 6.1.6, na página 108, e a proposição 6.1.8, na página seguinte.

DefiniÇão A.2.1. Seja $(G, \cdot)$ um grupo. Dada uma uniformidade $\mathscr{D}$ sobre $G$, denotaremos por $\mathscr{F}_{\mathscr{D}}$ o conjunto

$$
\left\{F \subseteq G: E_{F} \in \mathscr{D}\right\}
$$

Reciprocamente, dada uma coleção $\mathscr{F} \subseteq \mathcal{P}(G)$, denotaremos por $\mathscr{D}_{\mathscr{F}}$ o conjunto

$$
\left\{D \subseteq G \times G: \exists F \in \mathscr{F} \text { t.q. } E_{F} \subseteq D\right\} .
$$

ObSERVAÇÃo A.2.2. Sejam $(G, \cdot)$ um grupo e $\mathscr{D}$ uma uniformidade sobre $G$. Nessas condições, não é difícil mostrar que

$$
\mathscr{F}_{\mathscr{D}}=\left\{F \subseteq G: \exists D \in \mathscr{D} \text { invariante à esquerda em }(G, \cdot) \text { t.q. } A_{D} \subseteq F\right\},
$$

o que, por sua vez, evidencia uma certa simetria entre as definições de $\mathscr{D}_{\mathscr{F}}$ e de $\mathscr{F}_{\mathscr{D}}$. Observe que isso é o análogo, pra uniformidades, do que ocorre com $\mathcal{A}_{\mathcal{E}}$ e $\mathcal{E}_{\mathcal{A}}$ na definição 6.1 .9 (veja a observação 6.1.10). 
Estamos, agora, preparados para enunciar um dos grandes resultados desta seção, o qual nada mais é do que o análogo do teorema 6.1.11 no contexto das uniformidades invariantes à esquerda.

Teorema A.2.3. Sejam $(G, \cdot)$ um grupo, e $\in G$ o elemento neutro de $(G, \cdot), \mathcal{U}$ a coleção das uniformidades sobre $G$ invariantes à esquerda em $(G, \cdot)$, e $\mathcal{F}$ a coleção dos filtros $\mathscr{F}$ sobre $G$ tais que, para todo $F \in \mathscr{F}, e \in F, F^{-1} \in \mathscr{F}$ e existe $H \in \mathscr{F}$ tal que $H^{2} \subseteq F$. Nessas condições:

a) $\mathscr{D}_{\mathscr{F}} \in \mathcal{U}$ para todo $\mathscr{F} \in \mathcal{F}$;

b) $\mathscr{F}_{\mathscr{D}} \in \mathcal{F}$ para todo $\mathscr{D} \in \mathcal{U}$;

c) A função $\varphi: \mathcal{F} \longrightarrow \mathcal{U}$ tal que $\varphi(\mathscr{F})=\mathscr{D}_{\mathscr{F}}$ é uma bijeção, e $\varphi^{-1}: \mathcal{U} \longrightarrow \mathcal{F}$ é tal que $\varphi^{-1}(\mathscr{D})=\mathscr{F}_{\mathscr{D}}$.

Demonstração. a) Dado $\mathscr{F} \in \mathcal{F}$, definamos $\mathscr{B}_{\mathscr{F}}:=\left\{E_{F}: F \in \mathscr{F}\right\}$. Vamos mostrar que $\mathscr{B}_{\mathscr{F}}$ é base de uma uniformidade sobre $G$. Para isso, devemos provar que $\mathscr{B}_{\mathscr{F}}$ satisfaz as condições de a) a d) da proposição 3.1.7. E, para tanto, é suficiente notar que, para todos $F, F^{\prime} \subseteq G$,

$$
\begin{gathered}
F, F^{\prime} \in \mathscr{F} \Rightarrow F \cap F^{\prime} \in \mathscr{F} \Rightarrow E_{F \cap F^{\prime}} \in \mathscr{B}_{\mathscr{F}} \Rightarrow E_{F} \cap E_{F^{\prime}} \in \mathscr{B}_{\mathscr{F}}, \\
F \in \mathscr{F} \Rightarrow e \in F \Rightarrow\{e\} \subseteq F \Rightarrow E_{\{e\}} \subseteq E_{F} \underset{E_{\{e\}}=\Delta}{\Longrightarrow} \Delta \subseteq E_{F}, \\
F \in \mathscr{F} \Rightarrow F^{-1} \in \mathscr{F} \Rightarrow E_{F^{-1}} \in \mathscr{B}_{\mathscr{F}} \Rightarrow E_{F}{ }^{-1} \in \mathscr{B}_{\mathscr{F}},
\end{gathered}
$$

$\mathrm{e}$

$$
\begin{aligned}
F \in \mathscr{F} & \Rightarrow \exists H \in \mathscr{F} \text { t.q. } H^{2} \subseteq F \Rightarrow \exists H \in \mathscr{F} \text { t.q. } E_{H^{2}} \subseteq E_{F} \\
& \Rightarrow \exists H \in \mathscr{F} \text { t.q. } E_{H} \circ E_{H} \subseteq E_{F} \Rightarrow \exists B \in \mathscr{B}_{\mathscr{F}} \text { t.q. } B \circ B \subseteq E_{F} .
\end{aligned}
$$

Note ainda que, dadas a definição de $\mathscr{D}_{\mathscr{F}}$ e a observação 3.1 .5 , fica claro que a uniformidade gerada por $\mathscr{B}_{\mathscr{F}}$ não é outra senão $\mathscr{D}_{\mathscr{F}}$. E, sendo $E_{F}$ invariante à esquerda para todo $F \in \mathscr{F}$, disso concluímos, por fim, que $\mathscr{D}_{\mathscr{F}} \in \mathcal{U}$.

b) Dado $\mathscr{D} \in \mathcal{U}$, vamos mostrar que $\mathscr{F}_{\mathscr{D}}$ é um filtro sobre $G$ tal que, para todo $F \in \mathscr{F}_{\mathscr{D}}, e \in F$, $F^{-1} \in \mathscr{F}$ e existe $H \in \mathscr{F}$ tal que $H^{2} \subseteq F$.

Observe, de início, que, para todos $F, F^{\prime} \subseteq G$,

$$
\begin{gathered}
F \in \mathscr{F}_{\mathscr{D}} \Rightarrow E_{F} \in \mathscr{D} \Rightarrow \Delta \subseteq E_{F} \Rightarrow E_{\{e\}} \subseteq E_{F} \Rightarrow\{e\} \subseteq F \Rightarrow e \in F, \\
F, F^{\prime} \in \mathscr{F}_{\mathscr{D}} \Rightarrow E_{F}, E_{F^{\prime}} \in \mathscr{D} \Rightarrow E_{F} \cap E_{F^{\prime}} \in \mathscr{D} \Rightarrow E_{F \cap F^{\prime}} \in \mathscr{D} \Rightarrow F \cap F^{\prime} \in \mathscr{F}_{\mathscr{D}}, \\
\left(F \in \mathscr{F}_{\mathscr{D}} \text { e } F \subseteq F^{\prime}\right) \Rightarrow\left(E_{F} \in \mathscr{D} \text { e } E_{F} \subseteq E_{F^{\prime}}\right) \Rightarrow E_{F^{\prime}} \in \mathscr{D} \Rightarrow F^{\prime} \in \mathscr{F}_{\mathscr{D}},
\end{gathered}
$$

e

$$
F \in \mathscr{F}_{\mathscr{D}} \Rightarrow E_{F} \in \mathscr{D} \Rightarrow E_{F}^{-1} \in \mathscr{D} \Rightarrow E_{F^{-1}} \in \mathscr{D} \Rightarrow F^{-1} \in \mathscr{F}_{\mathscr{D}}
$$

Precisamos, agora, mostrar que, para todo $F \in \mathscr{F}_{\mathscr{D}}$, existe $H \in \mathscr{F}_{\mathscr{D}}$ tal que $H^{2} \subseteq F$. Para isso, observe que, dado $F \subseteq G$,

$$
F \in \mathscr{F}_{\mathscr{D}} \Rightarrow E_{F} \in \mathscr{D} \Rightarrow \exists B \in \mathscr{D} \text { invariante à esquerda t.q. } B \circ B \subseteq E_{F} .
$$

Mas, para todo $B \in \mathscr{D}$ invariante à esquerda,

$$
B=E_{A_{B}} \Rightarrow A_{B} \in \mathscr{F}_{\mathscr{D}}
$$


e

$$
B \circ B=E_{A_{B}} \circ E_{A_{B}}=E_{A_{B}{ }^{2}}
$$

E, sendo assim,

$$
\begin{aligned}
F \in \mathscr{F}_{\mathscr{D}} & \Rightarrow \exists B \in \mathscr{D} \text { invariante à esquerda t.q. } E_{A_{B}{ }^{2}} \subseteq E_{F} \\
& \Rightarrow \exists B \in \mathscr{D} \text { t.q. }\left(A_{B} \in \mathscr{F}_{\mathscr{D}} \text { e } A_{B}{ }^{2} \subseteq F\right) .
\end{aligned}
$$

c) Dado $\mathscr{D} \in \mathcal{U}$, note que, para todo $D \subseteq G \times G$,

$$
D \in \mathscr{D}_{\mathscr{F}_{\mathscr{D}}} \Rightarrow \exists F \in \mathscr{F}_{\mathscr{D}} \text { t.q. } E_{F} \subseteq D \Rightarrow \exists F \subseteq G \text { t.q. }\left(E_{F} \in \mathscr{D} \text { e } E_{F} \subseteq D\right) \Rightarrow D \in \mathscr{D} .
$$

Reciprocamente, dado $D \in \mathscr{D}$, podemos fixar $B \in \mathscr{D}$ invariante à esquerda em $(G, \cdot)$ tal que $B \subseteq D$ (pois, por hipótese, $\mathscr{D}$ é invariante à esquerda). Observe que

$$
\left(B \in \mathscr{D} \text { e } B=E_{A_{B}}\right) \Rightarrow A_{B} \in \mathscr{F}_{\mathscr{D}} .
$$

E, por sua vez,

$$
\left(A_{B} \in \mathscr{F}_{\mathscr{D}} \text { e } E_{A_{B}}=B \subseteq D\right) \Rightarrow D \in \mathscr{D}_{\mathscr{F}_{\mathscr{D}}} .
$$

Isso mostra que $D \in \mathscr{D}_{\mathscr{F} \mathscr{D}}$. E, sendo $D \in \mathscr{D}$ um conjunto arbitrário, disso concluímos que $\mathscr{D}_{\mathscr{F}}=\mathscr{D}$.

Analogamente, dado $\mathscr{F} \in \mathcal{F}$, temos que, para todo $F \subseteq G$,

$$
F \in \mathscr{F}_{\mathscr{D} \mathscr{F}} \Leftrightarrow E_{F} \in \mathscr{D}_{\mathscr{F}} \Leftrightarrow \exists F^{\prime} \in \mathscr{F} \text { t.q. } E_{F^{\prime}} \subseteq E_{F} \Leftrightarrow \exists F^{\prime} \in \mathscr{F} \text { t.q. } F^{\prime} \subseteq F \Leftrightarrow F \in \mathscr{F},
$$

do que segue que $\mathscr{F}=\mathscr{F}_{\mathscr{D}}$.

Dessa forma, se definirmos $\psi: \mathcal{U} \longrightarrow \mathcal{F}$ tal que $\psi(\mathscr{D})=\mathscr{F}_{\mathscr{D}}$, teremos que

$$
(\psi \circ \varphi)(\mathscr{F})=\psi(\varphi(\mathscr{F}))=\psi\left(\mathscr{D}_{\mathscr{F}}\right)=\mathscr{F}_{\mathscr{D} \mathscr{F}}=\mathscr{F}
$$

$\mathrm{e}$

$$
(\varphi \circ \psi)(\mathscr{D})=\varphi(\psi(\mathscr{D}))=\varphi\left(\mathscr{F}_{\mathscr{D}}\right)=\mathscr{D}_{\mathscr{F}_{\mathscr{D}}}=\mathscr{D}
$$

quaisquer que sejam $\mathscr{F} \in \mathcal{F}$ e $\mathscr{D} \in \mathcal{U}$, a partir do que concluímos que $\varphi$ é uma bijeção, e que $\varphi^{-1}=\psi$.

O teorema A.2.3 é um resultado bastante forte, e, por meio dele, é possível estabelecer uma conexão muito interessante entre o material deste apêndice e o que vimos sobre grupos topológicos no capítulo 3. Para isso, inicialmente recorde que, no capítulo 3, definimos a estrutura uniforme à esquerda de um grupo topológico $(G, \cdot \tau)$ como sendo a uniformidade sobre $G$ que tem como base a coleção $\mathscr{L}:=\left\{E_{V}: V \in \mathcal{V}\right\}$, em que $\mathcal{V}$ denota a coleção das vizinhanças de $e$ em $(G, \tau)$ e, para cada $V \in \mathcal{V}$,

$$
E_{V}:=\left\{(x, y) \in G \times G: x^{-1} y \in V\right\} .
$$

Observe que a notação $E_{V}$, utilizada acima, é compatível com a definição 6.1.6. Isso está longe de ser mera coincidência, sendo, na verdade, consequência de algo bastante profundo.

A observação acima é bastante sugestiva. Com sorte, ela pode levar o leitor a olhar com mais carinho para a coleção $\mathcal{V}$ das vizinhanças de $e$ em $(G, \tau)$. E, após alguns momentos de 
reflexão, talvez ele perceba que

$$
\mathcal{U}_{L}:=\left\{D \subseteq G \times G: \exists V \in \mathcal{V} \text { t.q. } E_{V} \subseteq D\right\}=\mathscr{D}_{\mathcal{V}}
$$

Ao se dar conta disso, e tendo em vista que $\mathcal{U}_{L}$ é invariante à esquerda, é natural que ele se pergunte se vale também que $\mathscr{F}_{\mathcal{U}_{L}}=\mathcal{V}$. A resposta é afirmativa, e, a seguir, vamos explicar o porquê.

Com efeito, não é difícil mostrar que $\mathcal{V}$ é um filtro sobre $G$. E, sendo $\mathscr{L}:=\left\{E_{V}: V \in \mathcal{V}\right\}$ uma base de $\mathcal{U}_{L}$, disso resulta que, para todo $F \subseteq G$,

$$
F \in \mathscr{F}_{\mathcal{U}_{L}} \Leftrightarrow E_{F} \in \mathcal{U}_{L} \Leftrightarrow \exists V \in \mathcal{V} \text { t.q. } E_{V} \subseteq E_{F} \Leftrightarrow \exists V \in \mathcal{V} \text { t.q. } V \subseteq F \Leftrightarrow F \in \mathcal{V}
$$

a partir do que concluímos que $\mathcal{V}=\mathscr{F}_{\mathcal{U}_{L}} \in \mathcal{F}$ (sendo $\mathcal{F}$ como no teorema A.2.3).

Esse resultado, o qual, por si só, já é bastante intrigante, fica ainda mais interessante quando analisamos o assunto do ponto de vista da topologia. Com efeito, se utilizado em conjunto com a proposição 3.3.9, ele nos permite, prontamente, concluir que

$$
\tau=\tau_{\mathcal{U}_{L}}=\tau_{\mathscr{D} \mathcal{V}}
$$

o que, por sua vez, é só uma outra forma de dizer que $\mathscr{D} v$ é a estrutura uniforme à esquerda associada a $\left(G, \cdot, \tau_{\mathscr{D}}\right)$. Tendo isso em vista, é natural perguntarmos se vale a recíproca. Isto é:

Será que, para todo $\mathscr{F} \in \mathcal{F}, \mathscr{D}_{\mathscr{F}}$ é a estrutura uniforme à esquerda associada a $\left(G, \cdot, \tau_{\mathscr{D}}\right)$ ?

O teorema a seguir nos mostra que sim!

Teorema A.2.4. Sejam $(G, \cdot)$ um grupo e e $\in G$ o elemento neutro de $(G, \cdot)$. Nessas condições, uma uniformidade $\mathscr{D}$ sobre $G$ será invariante à esquerda em $(G, \cdot)$ se, e somente se, for a estrutura uniforme à esquerda associada ao grupo topológico $\left(G, \cdot, \tau_{\mathscr{D}}\right)$.

Demonstração. $(\Rightarrow)$ Dada uma uniformidade $\mathscr{D}$ sobre $G$ invariante à esquerda em $(G, \cdot)$, consideremos o filtro

$$
\mathscr{F}_{\mathscr{D}}:=\left\{F \subseteq G: E_{F} \in \mathscr{D}\right\} .
$$

Observe que, sendo $\mathscr{D}$ invariante à esquerda, segue do teorema A.2.3 (e das ideias envolvidas em sua demonstração) que

$$
\mathscr{B}_{\mathscr{F}}:=\left\{E_{F}: F \in \mathscr{F}\right\}
$$

é uma base de $\mathscr{D}$. Vamos, agora, provar que $\mathscr{F}_{\mathscr{D}}$ é, simplesmente, a coleção $\mathcal{V}_{\mathscr{D}}$ das vizinhanças de $e$ em $\left(G, \tau_{\mathscr{D}}\right)$, a partir do que seguirá que $\mathscr{D}$ é a estrutura uniforme à esquerda associada a $\left(G, \cdot, \tau_{\mathscr{D}}\right)$.

Para isso, inicialmente, fixemos $F \in \mathscr{F}_{\mathscr{D}}$ de modo arbitrário. Como

$$
\mathcal{U}:=\{D[e]: D \in \mathscr{D}\}
$$

é uma base local de e em $\left(G, \tau_{\mathscr{D}}\right)$, para concluírmos que $F \in \mathcal{V}_{\mathscr{D}}$, basta mostrarmos que existe $D \in \mathscr{D}$ tal que $D[e] \subseteq F$. E, para tanto, é suficiente notar que $E_{F} \in \mathscr{D}$ (pois $F \in \mathscr{F}_{\mathscr{D}}$ ) e que

$$
E_{F}[e]:=\left\{x \in G:(e, x) \in E_{F}\right\}=A_{E_{F}}=F .
$$

Tomemos, agora, um conjunto $V \in \mathcal{V}_{\mathscr{D}}$ qualquer, e vamos mostrar que $V \in \mathscr{F}_{\mathscr{D}}$. Para começar, note que, sendo $\mathcal{U}$ uma base local de $e$ em $\left(G, \tau_{\mathscr{D}}\right)$, podemos fixar $B \in \mathscr{D}$ de modo que $B[e] \subseteq V$. E, como $\mathscr{D}$ é invariante à esquerda, podemos, também, assumir que $B$ é invariante 
à esquerda. Observe que, nesse caso, o conjunto $A_{B}$ está bem definido. Além disso, é fácil ver que $A_{B} \in \mathscr{F}_{\mathscr{D}}($ pois $B \in \mathscr{D})$ e que

$$
A_{B}=E_{A_{B}}[e]=B[e] \subseteq V .
$$

E, sendo $\mathscr{F}_{\mathscr{D}}$ um filtro sobre $G$, disso concluímos que $V \in \mathscr{F}_{\mathscr{D}}$.

$(\Leftarrow)$ É óbvio, uma vez que, como vimos, a estrutura uniforme à esquerda associada a um grupo topológico é sempre invariante à esquerda.

O teorema A.2.4 tem o seguinte corolário:

Corolário A.2.5. Sejam $(G, \cdot)$ e $\mathcal{F}$ como no teorema A.2.3. Nessas condições, se $\mathscr{F} \in \mathscr{F}$, então $\mathscr{F}$ coincide com a coleção das vizinhanças de e em $\left(G, \tau_{\mathscr{D}}\right)$.

Demonstração. Seja $\mathscr{F} \in \mathcal{F}$ um filtro dado. Como $\mathscr{F} \in \mathcal{F}$, segue de A.2.3 que $\mathscr{D} \mathscr{F}$ é uma uniformidade sobre $G$ invariante à esquerda em $(G, \cdot)$. E, sendo assim, podemos lançar mão dos mesmos argumentos utilizados na demonstração de A.2.4 para concluir que $\mathscr{F}=\mathscr{F}_{\mathscr{D} \mathscr{F}}$ é a coleção das vizinhanças de $e \operatorname{em}\left(G, \tau_{\mathscr{D}}\right)$. 


\section{Referências Bibliográficas}

[Bir36] G. Birkhoff. A note on topological groups. Compositio Math, 3:427-430, 1936. 113

[Bou66] N. Bourbaki. General Topology, part 1. Addison-Wesley Publishing Company, 1966. Trad. 29

[Kal08] N. Kalton. The Nonlinear Geometry of Banach Spaces. Rev. Mat. Complut., 21(1):760, 2008. 3, 89

[Kal12] N. Kalton. The uniform structure of Banach spaces. Math. Ann., 354(4):1247-1288, 2012. 89

[Roe03] J. Roe. Lectures on Coarse Geometry. Em University Lecture Series, volume 31. American Mathematical Society, 2003. 1, 53

[Ros17a] C. Rosendal. Coarse Geometry of Topological Groups. Manuscrito, 2017. 1, 4, 6, 63, $105,115,116$

[Ros17b] C. Rosendal. Equivariant geometry of Banach spaces and topological groups. Forum of Mathematics, Sigma, 5(22), 2017. Disponível em: http://www.cambridge.org/core. $3,63,81,89,104$

[Wei37] A. Weil. Sur les Espaces à Structure Uniforme et sur la Topologie Générale. Hermann, primeira edição, 1937. 29, 63

[Wil70] S. Willard. General Topology. Addison-Wesley Publishing Company, primeira edição, 1970. 29, 39

[Wri02] N. Wright. $C_{0}$ coarse geometry. Tese de Doutorado, Penn State, 2002. 60

[Wri03] N. Wright. $\mathrm{C}_{0}$ coarse geometry and scalar curvature. Journal of Functional Analysis, 197(2):469-488, 2003. 60 


\section{Índice Remissivo}

B

Base

de um espaço topológico, 21

de uma estrutura coarse, 55

de uma uniformidade, 31

local, 20

Bola

aberta, 8

fechada, 8

Bornologia, 64

C

$c_{0}, 13$

Composição de relações binárias, 6

Conjunto

colimitado, 73

compacto, 20

controlado, 54

fechado, 19

gerador, 14, 26

grosseiramente limitado, 112

Continuidade, 10, 24, 47

uniforme, 11, 47

Convergência, 9

uniforme, 11

Critério da comparação, 12

D

Diâmetro de um conjunto, 8

Dimensão infinita, 14

E

Equivalência

grosseira, 72

uniforme, 51

Espaço

coarse, 54

de Banach, 16

de Hausdorff, 19

métrico, 8

completo, 10 pseudométrico, 8

topológico, 18

separável, 19

uniformizável, 44

uniforme, 31

vetorial, 12

finitamente gerado, 14

normado, 16

Espaços

grosseiramente equivalentes, 73

uniformemente equivalentes, 51

uniformemente homeomorfos, 51

uniformemente isomorfos, 51

Estrutura

coarse, 54

$\mathcal{C}_{0}, 60$

à esquerda, 64

discreta, 56

gerada por uma coleção, 62

indiscreta, 57

invariante à esquerda, 105

limitada associada a uma pseudométrica, 58

maximal, 56

minimal, 56

uniforme, 30

à direita, 39

à esquerda, 38

induzida por uma pseudométrica, 35

invariante à esquerda, 125

Estruturas uniformes equivalentes, 39

Expansividade, 67

F

Fecho, 19

Filtro, 25

principal gerado por $x, 25$

Função

bornológica, 64

colimitada, 74 
contínua, 10, 24, 47

expansível, 67

não colapsada, 79

uniformemente contínua, 11, 47

Funções

próximas, 69

G

Grupo, 26

abeliano, 26

finitamente gerado, 26

topológico, 27

pseudometrizável, 39

$\mathbf{H}$

Homeomorfismo, 24

uniforme, 51

\section{I}

Ideal, 25

Interior, 19

Inversa de uma relação binária, 6

Isomorfismo uniforme, 51

\section{K}

$\mathbb{K}$ - espaço vetorial, 12

$\mathbb{K}^{\mathbb{N}}, 13$

\section{L}

$\ell_{\infty}, 13$

$\ell_{p}, 13$

$\ell_{p}(E,\|\cdot\|), 84$

\section{M}

Métrica, 8

da palavra, 41

discreta, 8

induzida por uma norma, 16

usual sobre $\mathbb{K}, 8$

Mergulho

grosseiro, 73

uniforme, 49

$\mathrm{N}$

Núcleo de uma tranformação linear, 15

Norma, 15

de uma tranformação linear, 17

Normas equivalentes, 24

\section{$\mathrm{O}$}

Operador linear, 14
$\mathbf{P}$

Ponto

aderente, 19

interior, 19

Projeção, 15

Pseudométrica, 7

compatível, 18

contínua em um grupo topológico, 63

invariante à esquerda, 40

trivial, 8

Pseudométricas equivalentes, 23

$\mathbf{R}$

Relação

binária, 5

diagonal, 5

simétrica, 6

de equivalência, 23, 51, 69, 73, 79

de proximidade, 69

$\mathrm{S}$

Sequência

convergente, 9

de Cauchy, 9

limitada, 9

Subconjunto

denso, 19

invariante à esquerda, 26

limitado, 122

próprio, 56

relativamente compacto, 20

Subespaço

coarse, 56

gerado, 14

topológico, 18

uniforme, 34

vetorial, 14

Subgrupo, 26

$\mathbf{T}$

Topologia, 18

caótica, 18

discreta, 18

induzida pela norma, 19

induzida por uma pseudométrica, 18

induzida por uma uniformidade, 44

produto, 23

pseudometrizável, 18

Transformação linear, 14

limitada, 17 
U

Uniformidade, 30

à esquerda, 38

discreta, 32

induzida por uma pseudométrica, 35

trivial, 32

usual sobre $\mathbb{R}, 32$

V

Vetor, 13

Vizinhança, 19

diagonal, 30 